Supporting Information

\title{
Inter/intramolecular Cascade of 1,6-Enynes Catalyzed by AllMetal Aromatic Tripalladium Complexes and Carboxylic Acids
}

\author{
Andrea Serafino, Nicola Camedda, Matteo Lanzi, Nicola Della Ca', Gianpiero Cera and \\ Giovanni Maestri* \\ Università di Parma, Dipartimento di Scienze Chimiche, della Vita e della Sostenibilità Ambientale, Parco Area
} delle Scienze 17/A, 43124 Parma, Italy

giovanni.maestri@unipr.it

\section{TABLE OF CONTENTS}

1. Scope limitations $\quad$ S2

2. Copies of NMR Spectra S3 


\section{Scope limitations}<smiles>C#CCN([TeH])[C@@H](C)/C=C/c1ccccc1</smiles><smiles>C/C(=C\c1ccccc1)COCC#CCOC/C=C/c1ccccc1</smiles><smiles>C#CC(OC/C=C/c1ccccc1)c1ccccc1</smiles><smiles>C#CCN([Te])[C@H](/C=C/c1ccccc1)c1ccccc1</smiles><smiles>C#CCN(C/C=C/c1ccccc1)S(=O)(=O)c1ccc(I)cc1</smiles><smiles>Brc1ccc(/C=C/COCC#CCOC/C=C/c2ccccc2)cc1</smiles><smiles>C=C=CCN([As])CC#CCOCC=Cc1ccccc1</smiles><smiles>C#CC(OC(C=C)c1ccccc1)c1ccccc1</smiles> 
2. Copies of NMR spectra 
1a, ${ }^{1} \mathbf{H}$ NMR $\left(300 \mathrm{MHz}, \mathrm{CDCl}_{3}\right)$

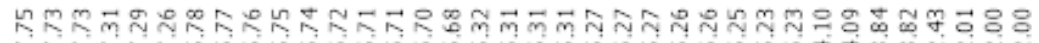

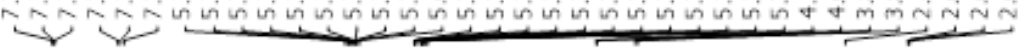

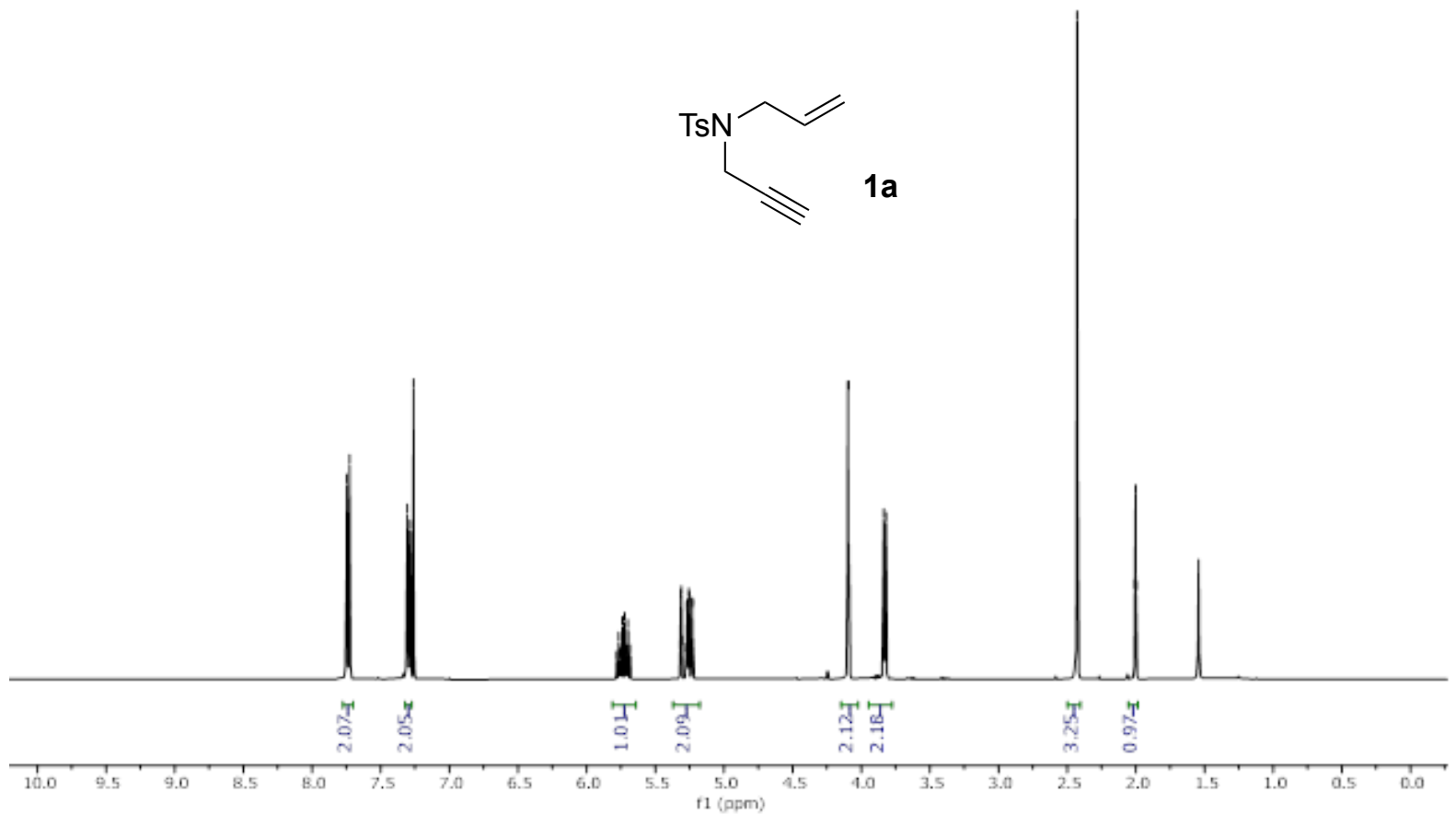

1a, ${ }^{13} \mathbf{C}\{\mathbf{H}\}$ NMR $\left(101 \mathrm{MHz}, \mathrm{CDCl}_{3}\right)$
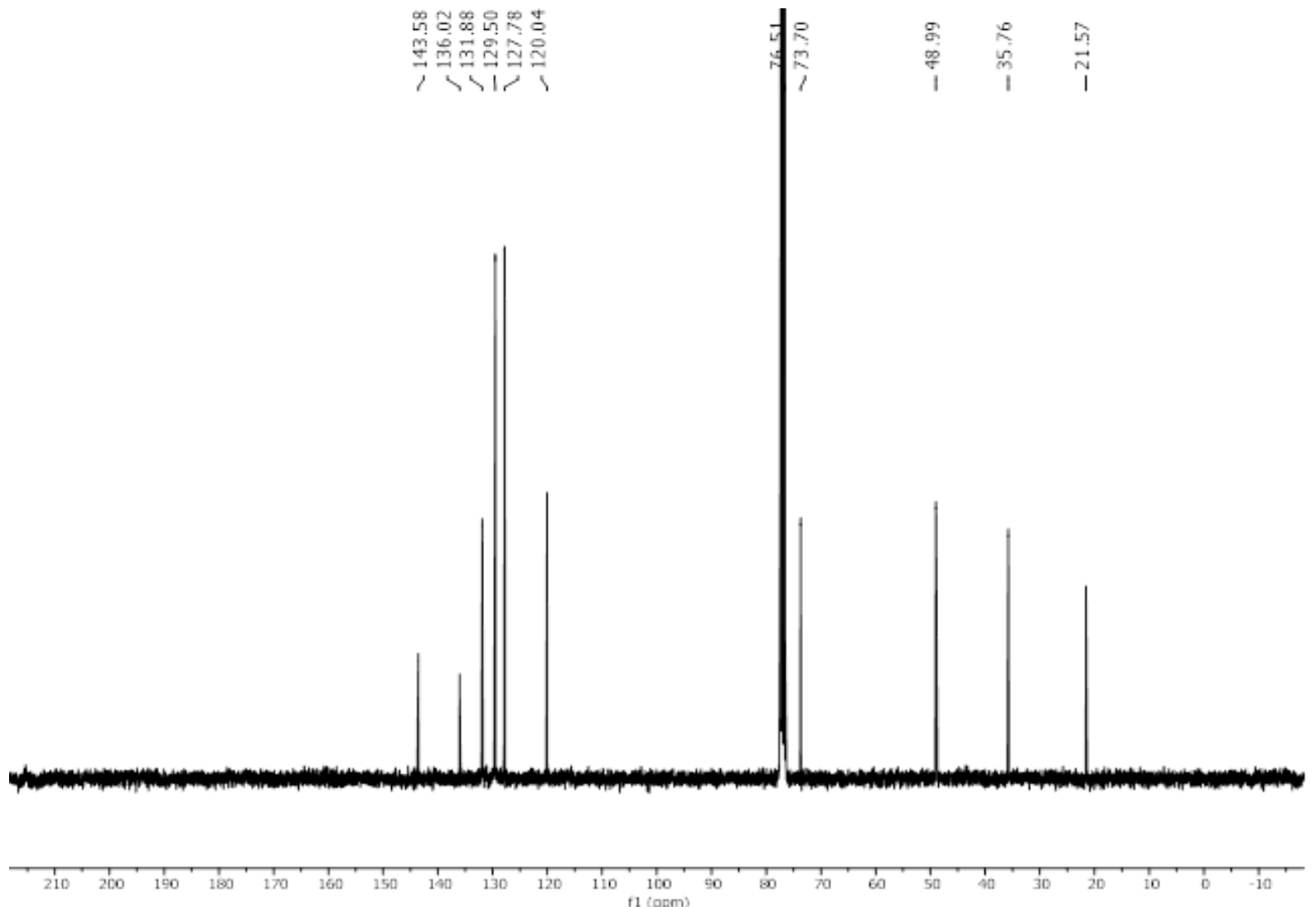
1b, ${ }^{1} \mathbf{H}$ NMR $\left(300 \mathrm{MHz}, \mathrm{CDCl}_{3}\right)$

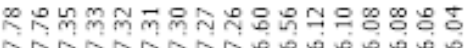

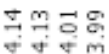

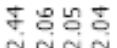

$4<$

$\overbrace{1 b}^{N^{-T s}}$
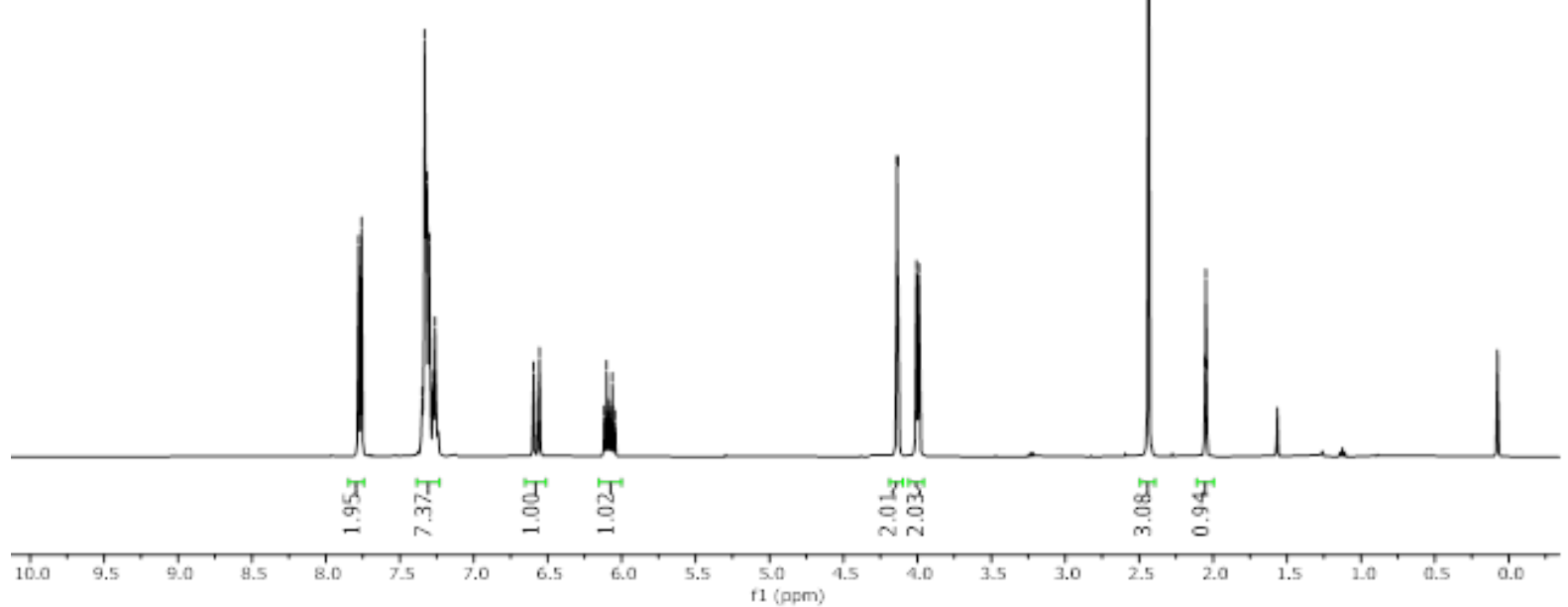

1b, ${ }^{13} \mathbf{C}\{\mathbf{H}\}$ NMR $\left(101 \mathrm{MHz}, \mathrm{CDCl}_{3}\right)$

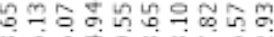

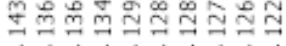

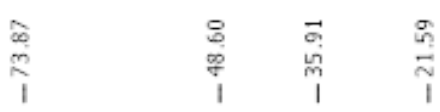

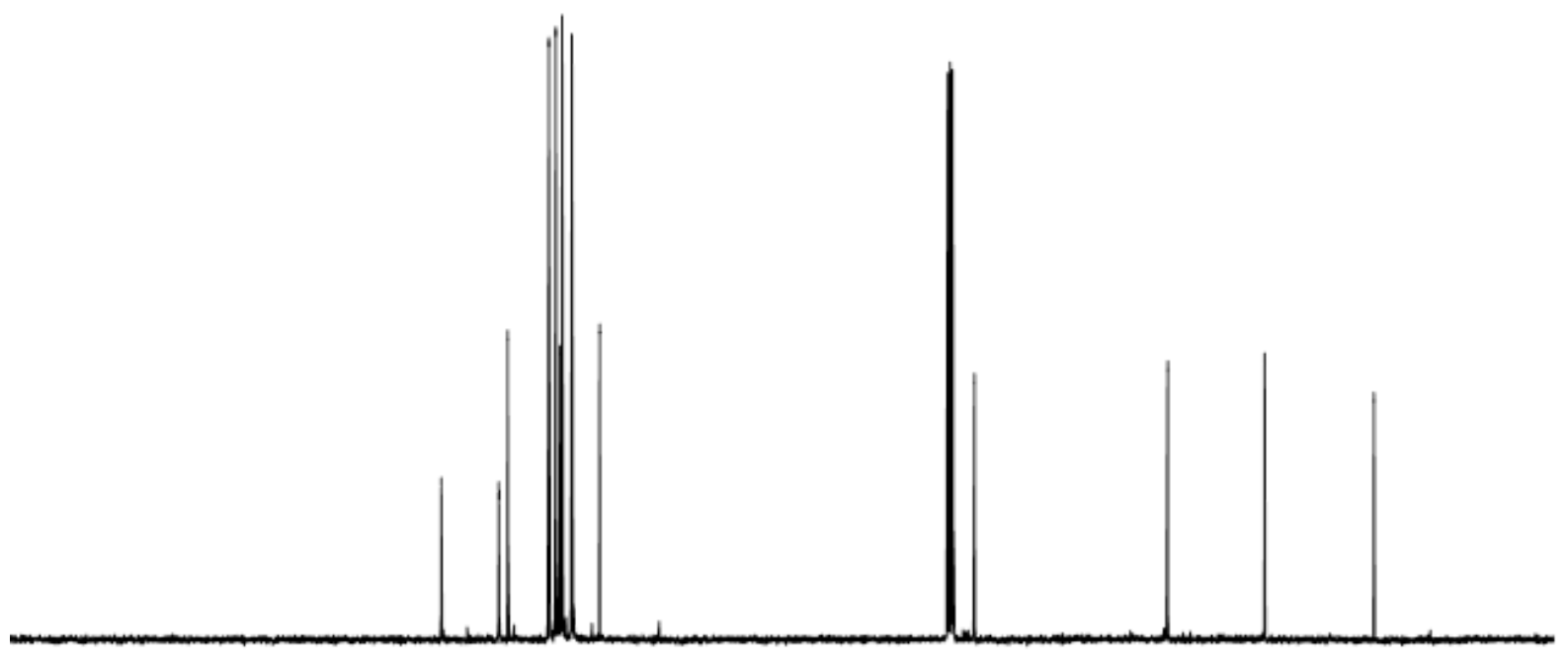

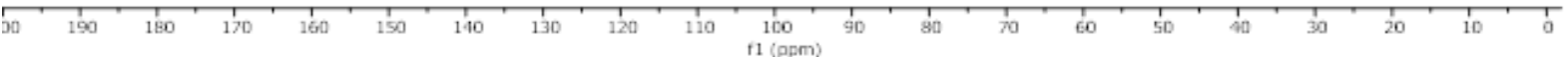


1c, ${ }^{1} \mathbf{H}$ NMR $\left(400 \mathrm{MHz}, \mathrm{CDCl}_{3}\right)$
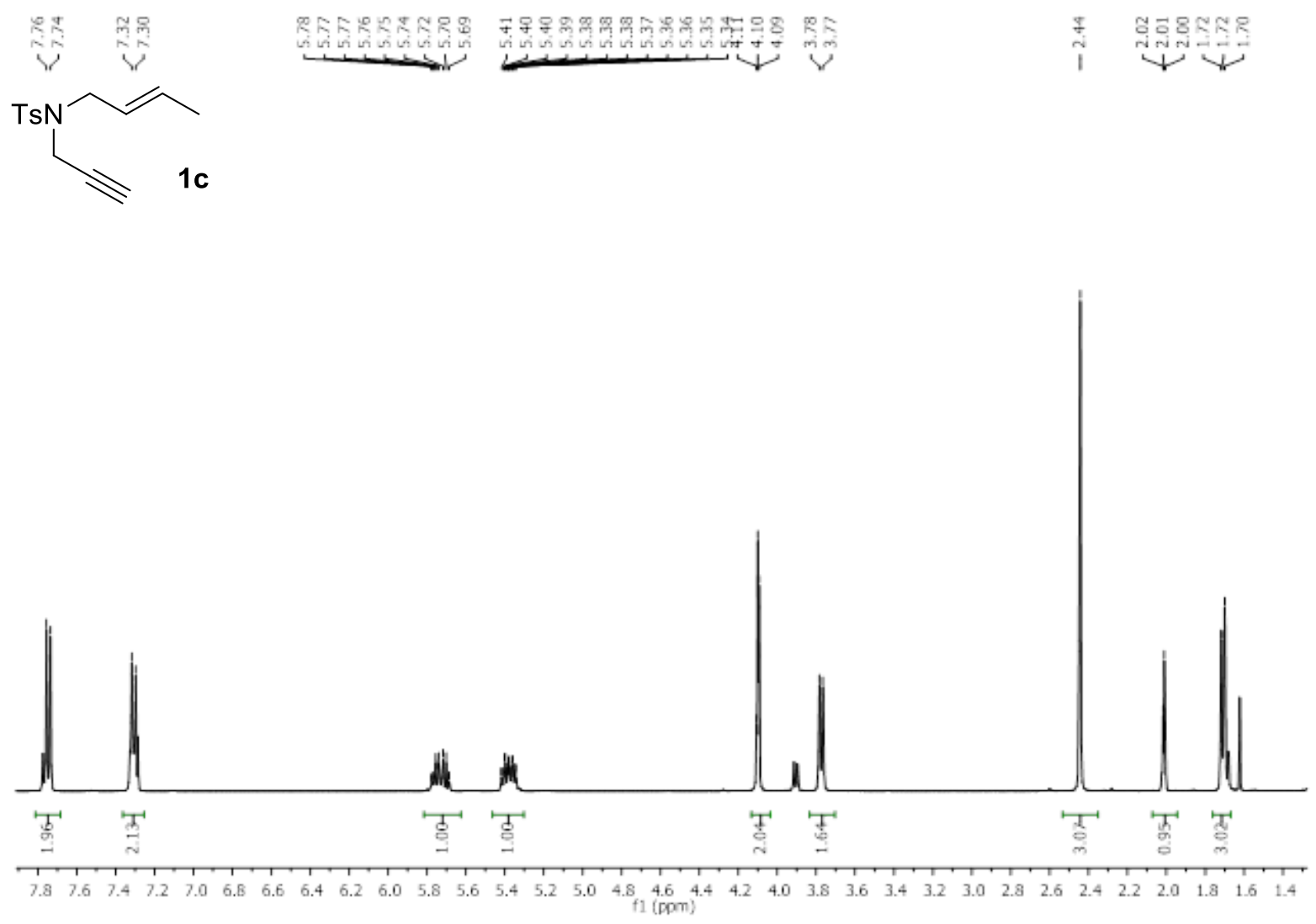
1d, ${ }^{1} \mathbf{H}$ NMR $\left(400 \mathrm{MHz}, \mathrm{CDCl}_{3}\right)$

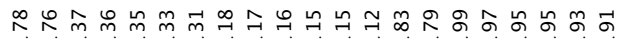

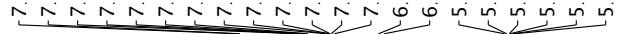

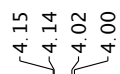

$\forall \vec{m}$ ำ

$\overbrace{1 d}^{N-T s}$

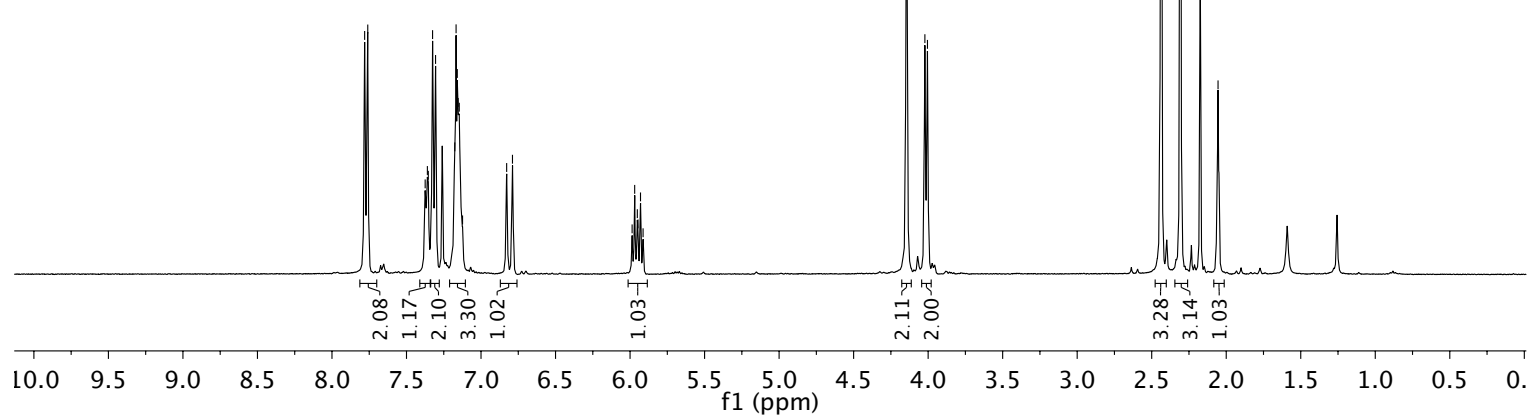


1e, ${ }^{1} \mathbf{H}$ NMR $\left(400 \mathrm{MHz}, \mathrm{CDCl}_{3}\right)$

œ

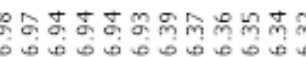

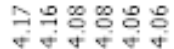

ชาำำำ

$\sqrt[4]{\mathrm{N} y}$<smiles>C#CCN([I-])C/C=C/c1ccccc1C#N</smiles>

$1 e$
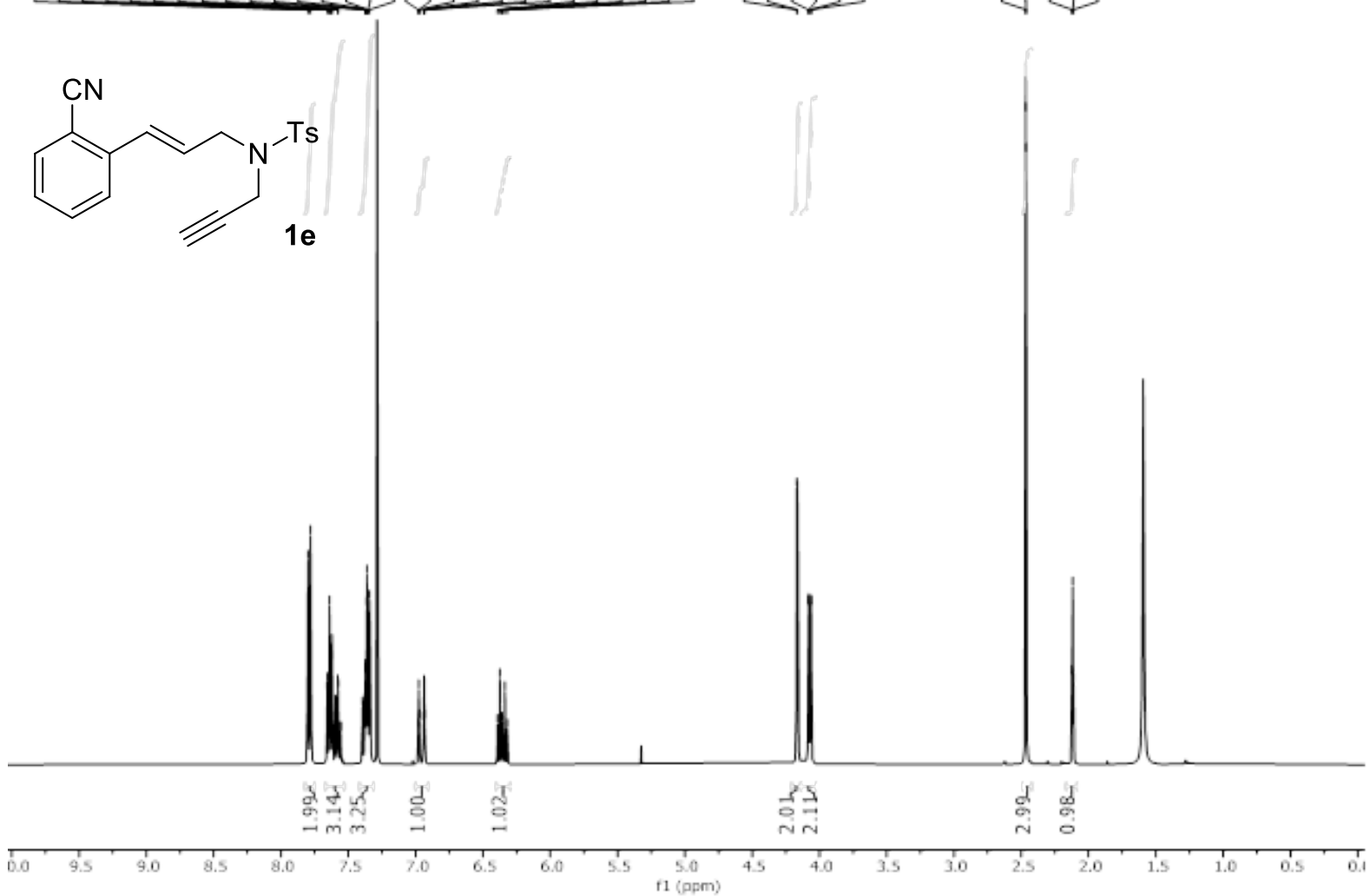

1e, ${ }^{13} \mathbf{C}\{\mathbf{H}\}$ NMR $\left(101 \mathrm{MHz}, \mathrm{CDCl}_{3}\right)$

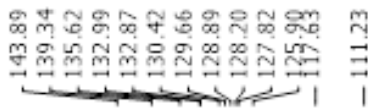

\begin{tabular}{|c|c|c|}
\hline ช. & $\begin{array}{l}\infty \\
\substack{0 \\
\infty \\
\infty}\end{array}$ & 5 \\
\hline 2 & 1 & 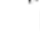 \\
\hline
\end{tabular}

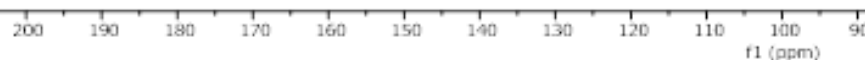


1f, ${ }^{1} \mathbf{H}$ NMR (400 MHz, $\left.\mathrm{CDCl}_{3}\right)$

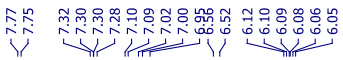

꾹울우요요

i $\stackrel{\text { i }}{\text { i }}$
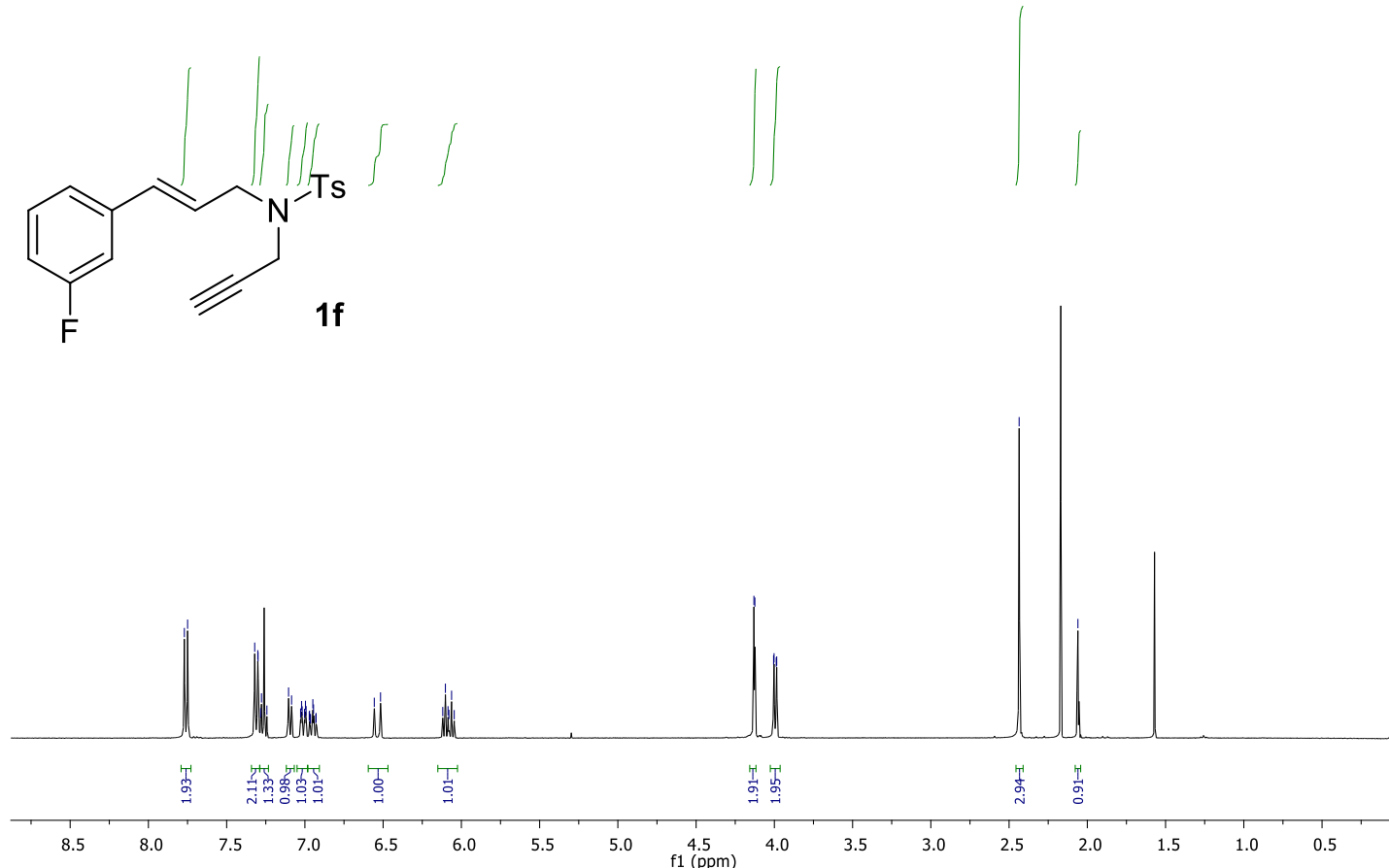
1g, ${ }^{1} \mathbf{H}$ NMR $\left(400 \mathrm{MHz}, \mathrm{CDCl}_{3}\right)$

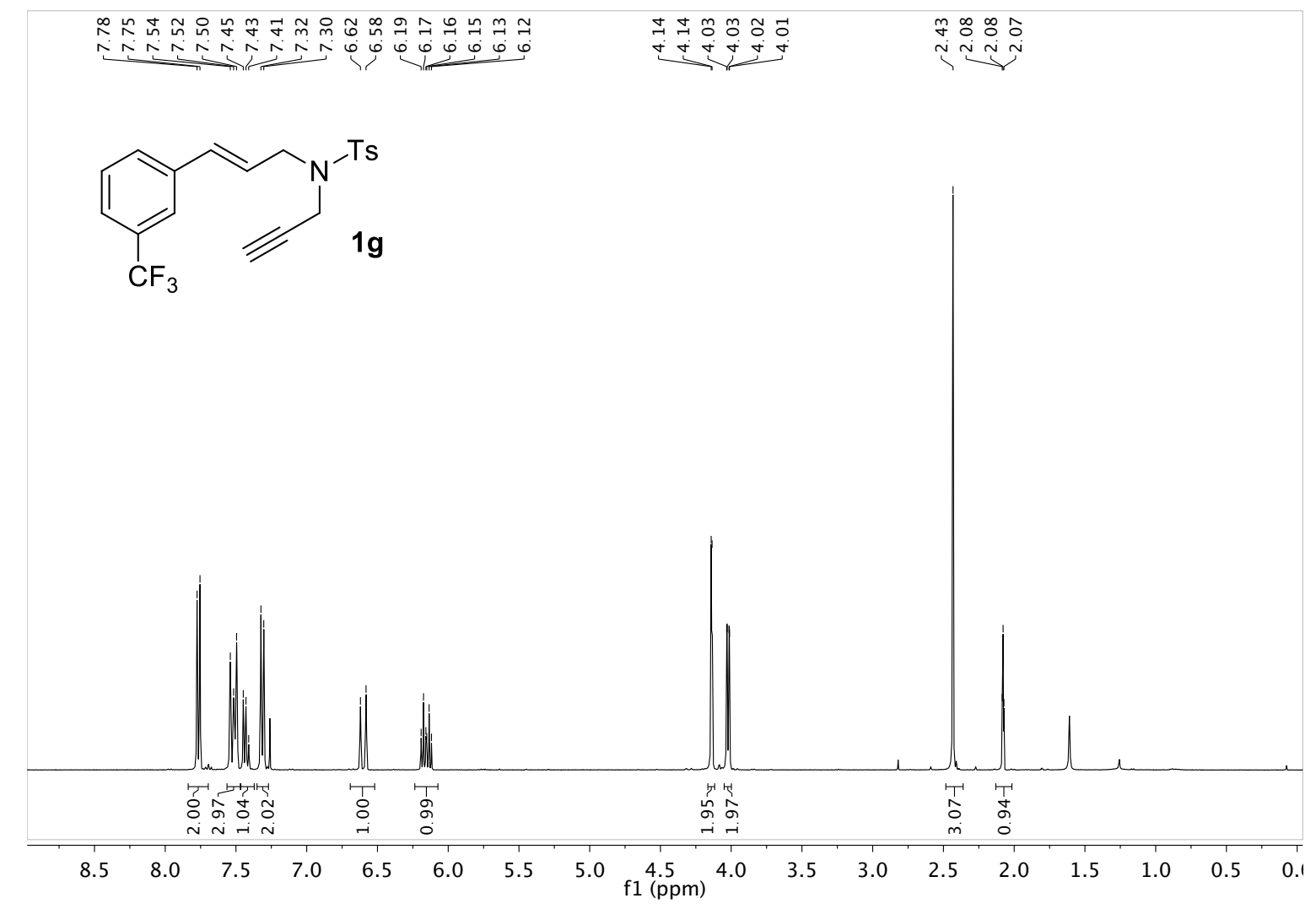

1g, ${ }^{13} \mathbf{C}\{\mathbf{H}\}$ NMR $\left(101 \mathrm{MHz}, \mathrm{CDCl}_{3}\right)$

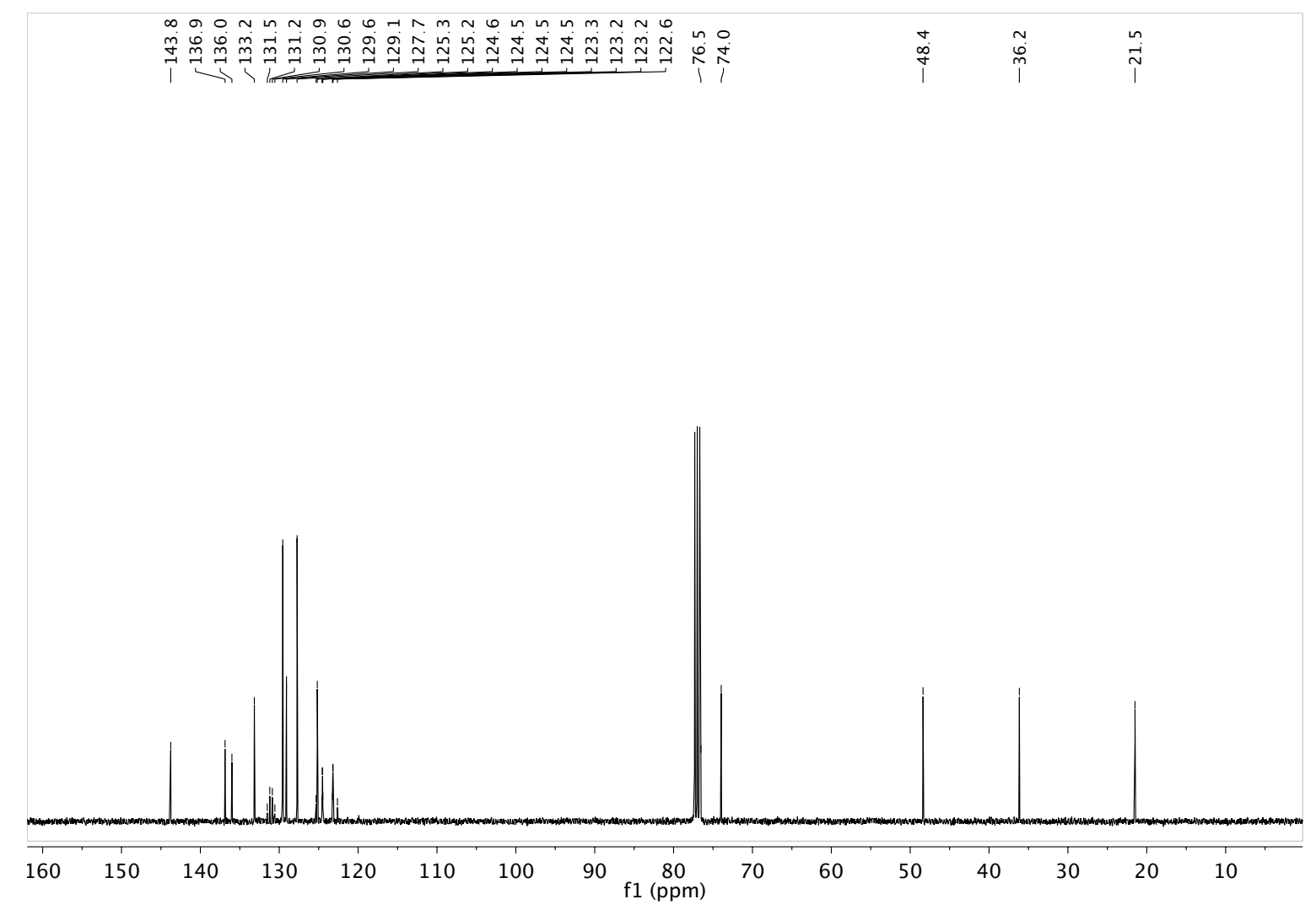


1g, ${ }^{19} \mathbf{F}\{\mathbf{H}\} \mathbf{N M R}\left(376 \mathrm{MHz}, \mathrm{CDCl}_{3}\right)$<smiles>C#CCN([135I])C/C=C/c1cccc(C(F)(F)F)c1</smiles>

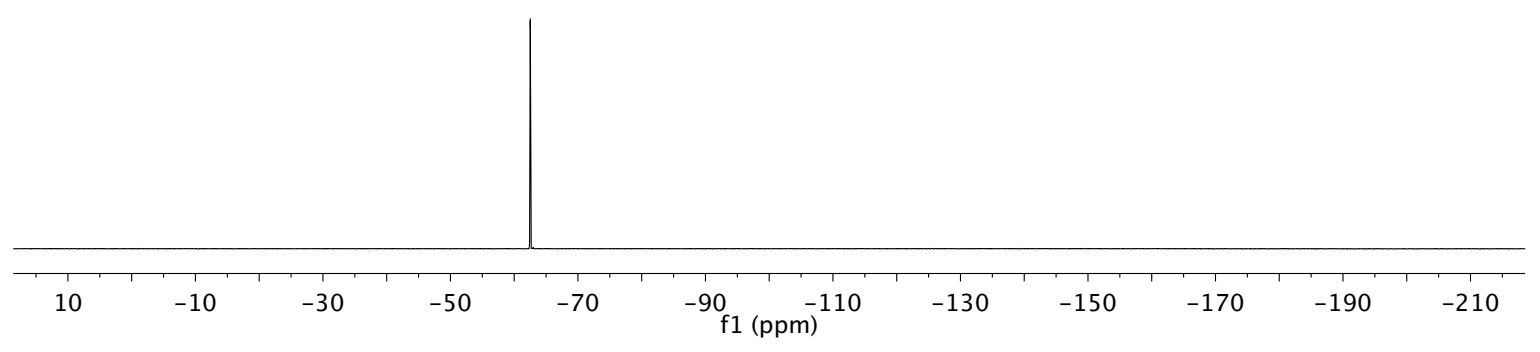


1h, ${ }^{1} \mathbf{H}$ NMR $\left(400 \mathrm{MHz}, \mathrm{CDCl}_{3}\right)$

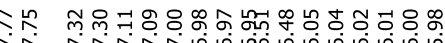

N 00 a 0000000

ๆำริ
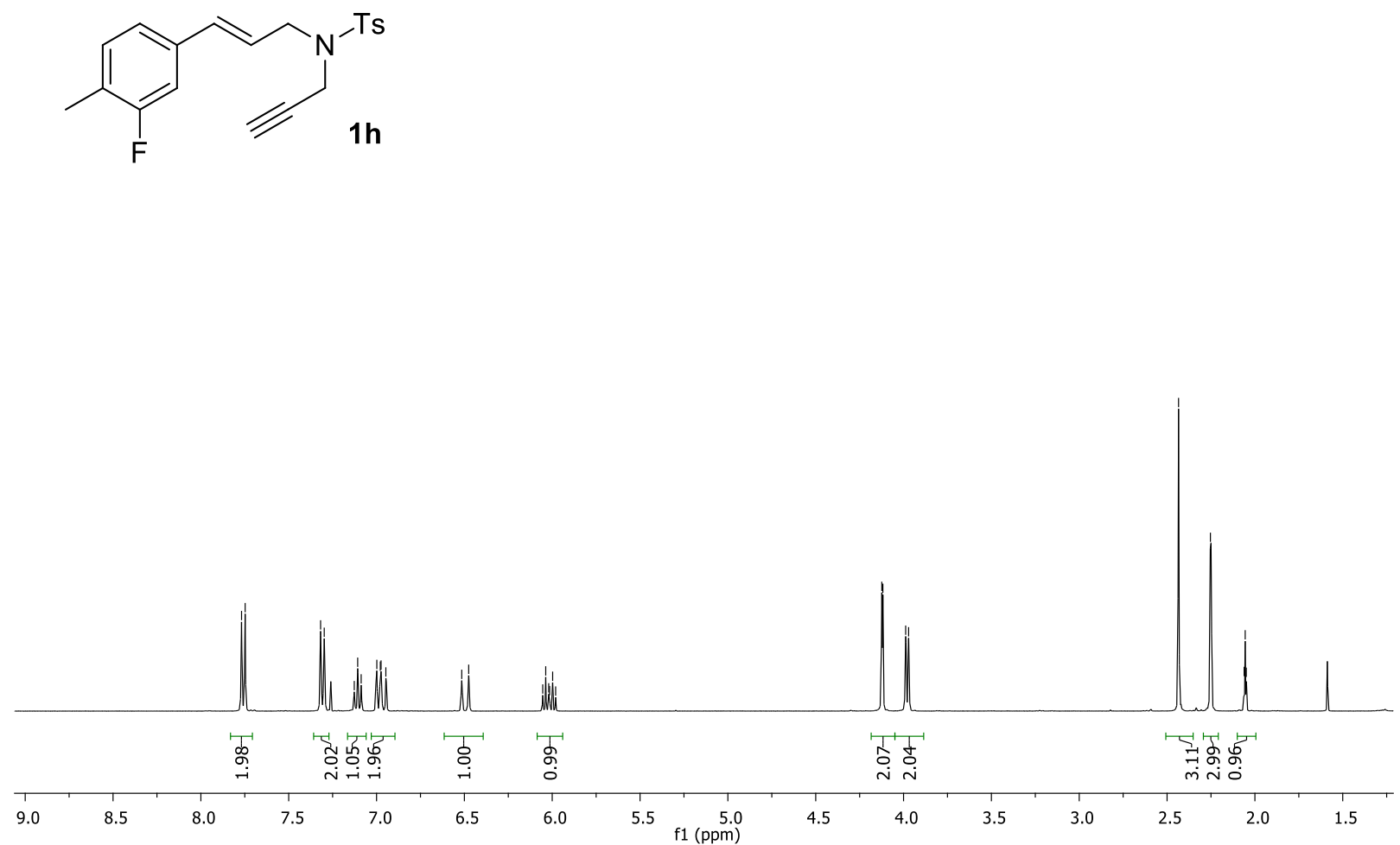

1h, ${ }^{13} \mathbf{C}\{\mathbf{H}\}$ NMR $\left(101 \mathrm{MHz}, \mathrm{CDCl}_{3}\right)$

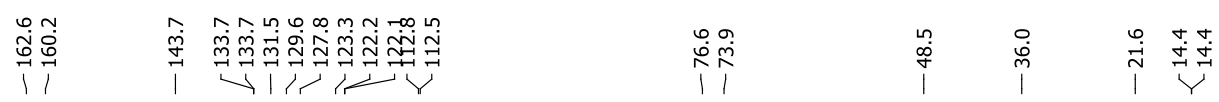

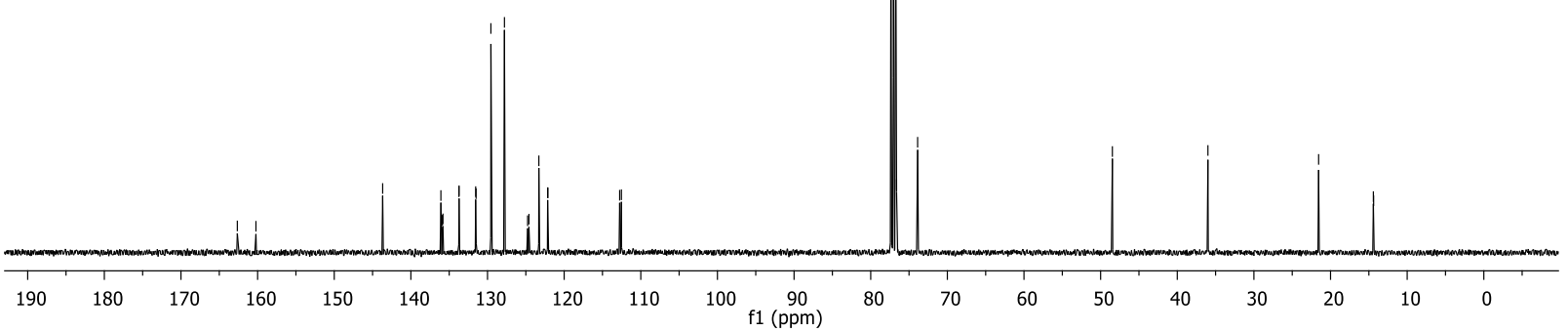


1h, ${ }^{19} \mathbf{F}\{\mathbf{H}\}$ NMR $\left(376 \mathrm{MHz}, \mathrm{CDCl}_{3}\right)$

$$
\stackrel{0}{\stackrel{1}{i}}
$$<smiles>C#CCN([AlH2])C/C=C/c1ccc(C)c(F)c1</smiles>

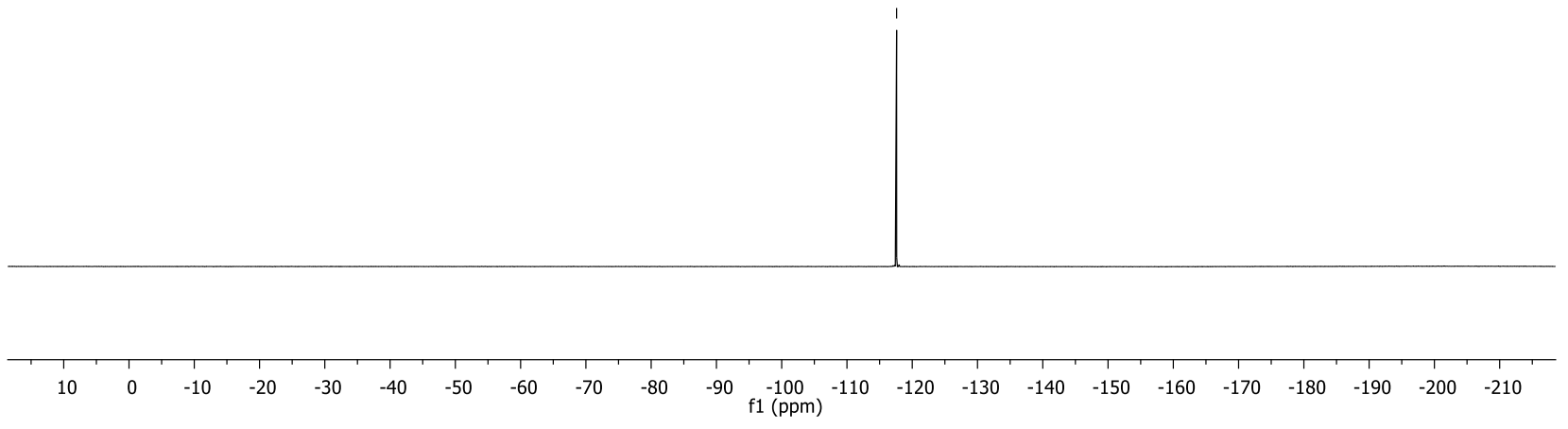


1i, ${ }^{1} \mathbf{H}$ NMR (300 MHz, $\left.\mathrm{CDCl}_{3}\right)$

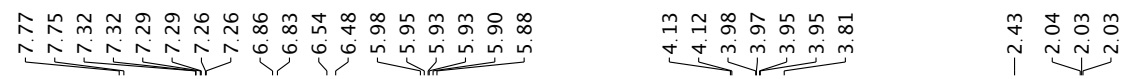<smiles>C#CCN([AlH2])C/C=C/c1ccc(OC)cc1</smiles>

$1 \mathrm{i}$

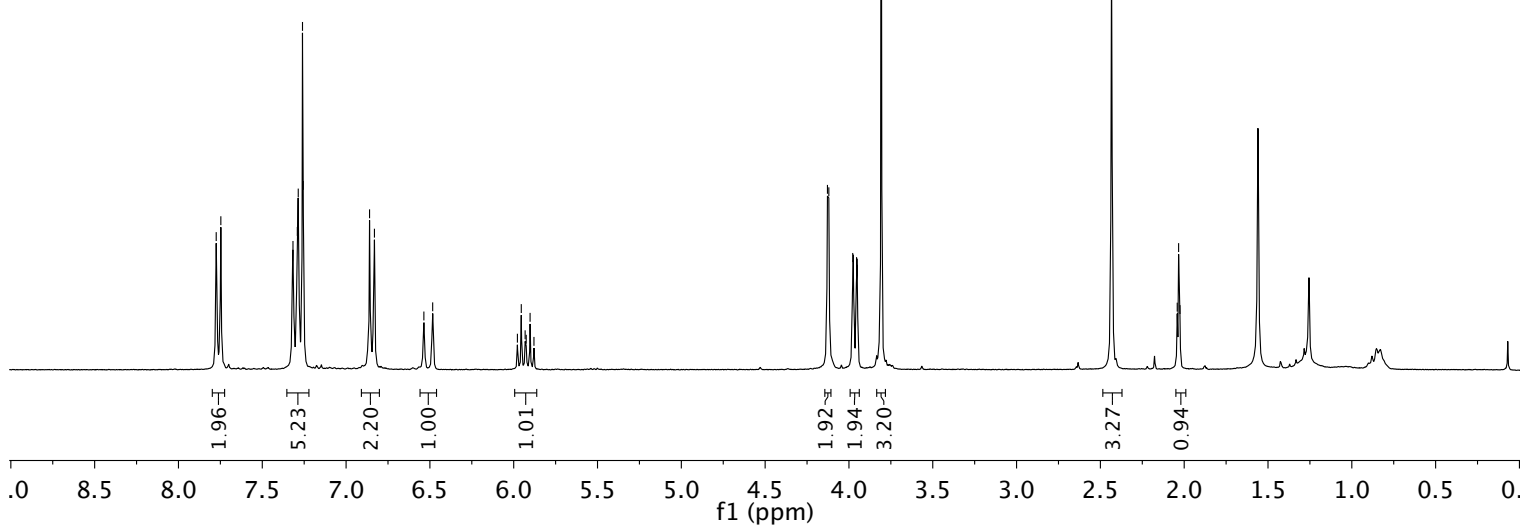

1i, ${ }^{13} \mathbf{C}\{\mathbf{H}\}$ NMR $\left(75 \mathrm{MHz}, \mathrm{CDCl}_{3}\right)$

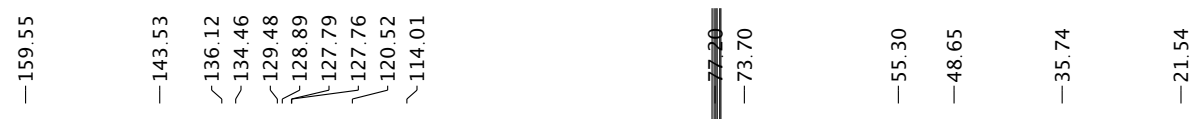

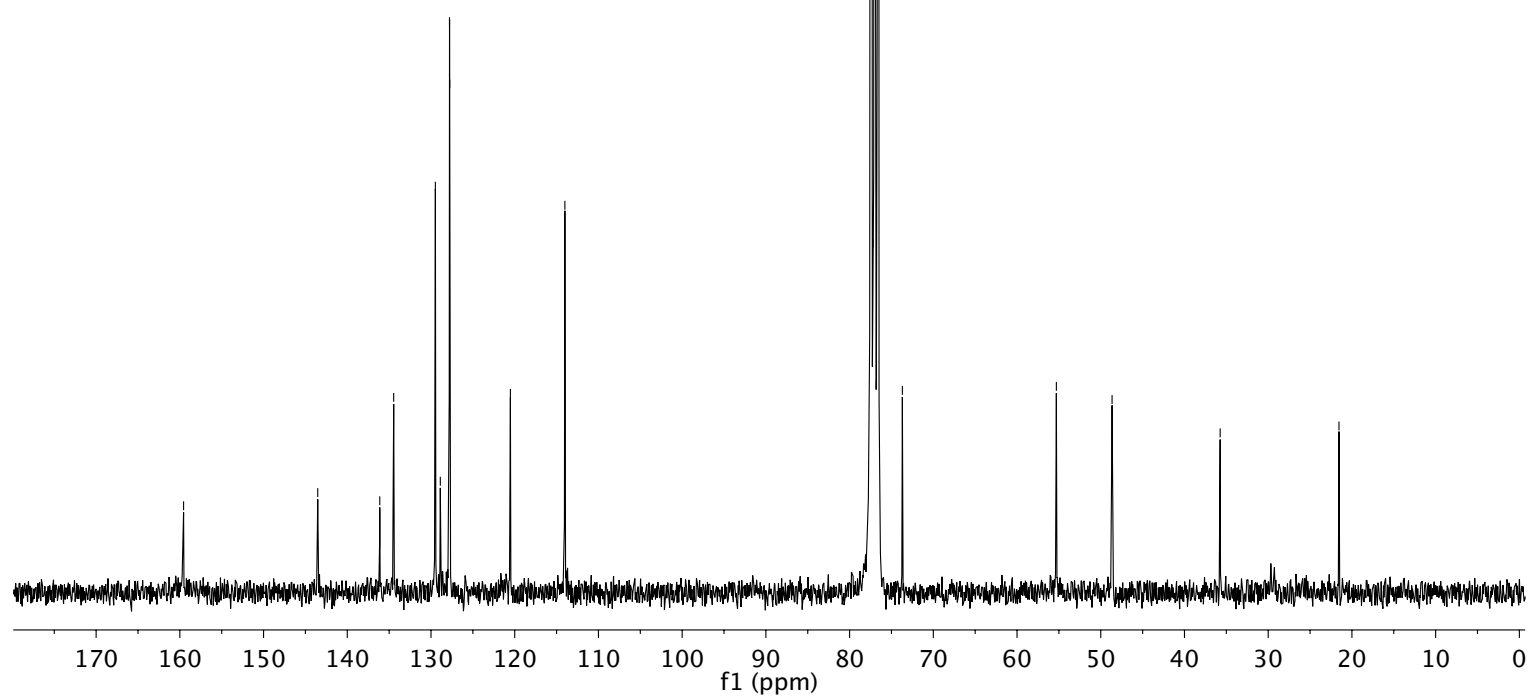


1j, ${ }^{1} \mathbf{H}$ NMR $\left(300 \mathrm{MHz}, \mathrm{CDCl}_{3}\right)$

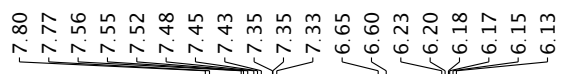

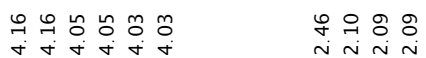

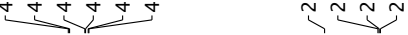

ij

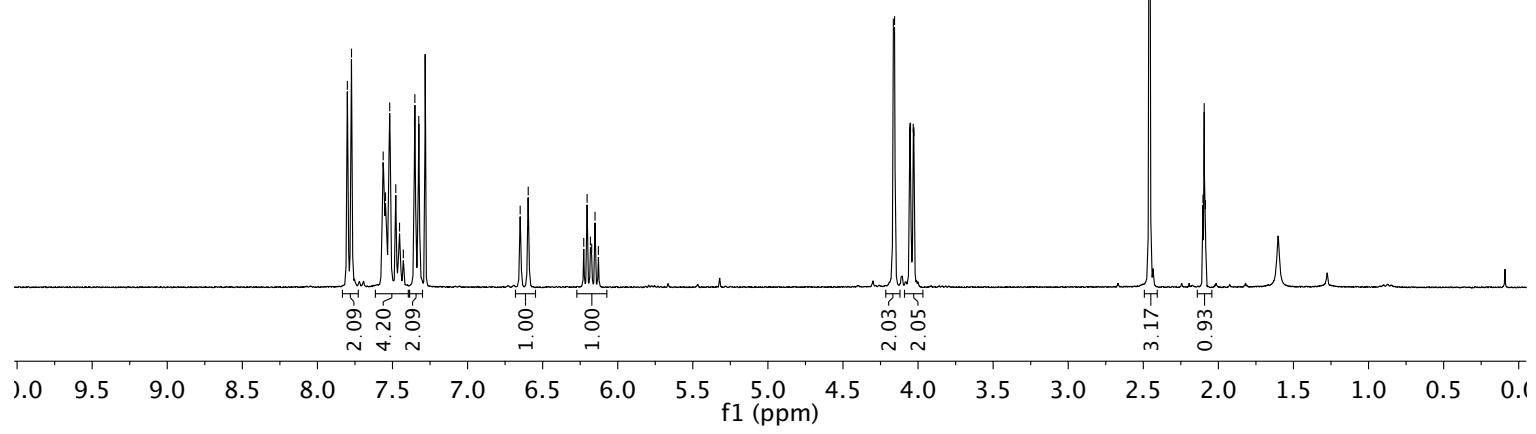


1k, ${ }^{1} \mathbf{H}$ NMR $\left(300 \mathrm{MHz}, \mathrm{CDCl}_{3}\right)$
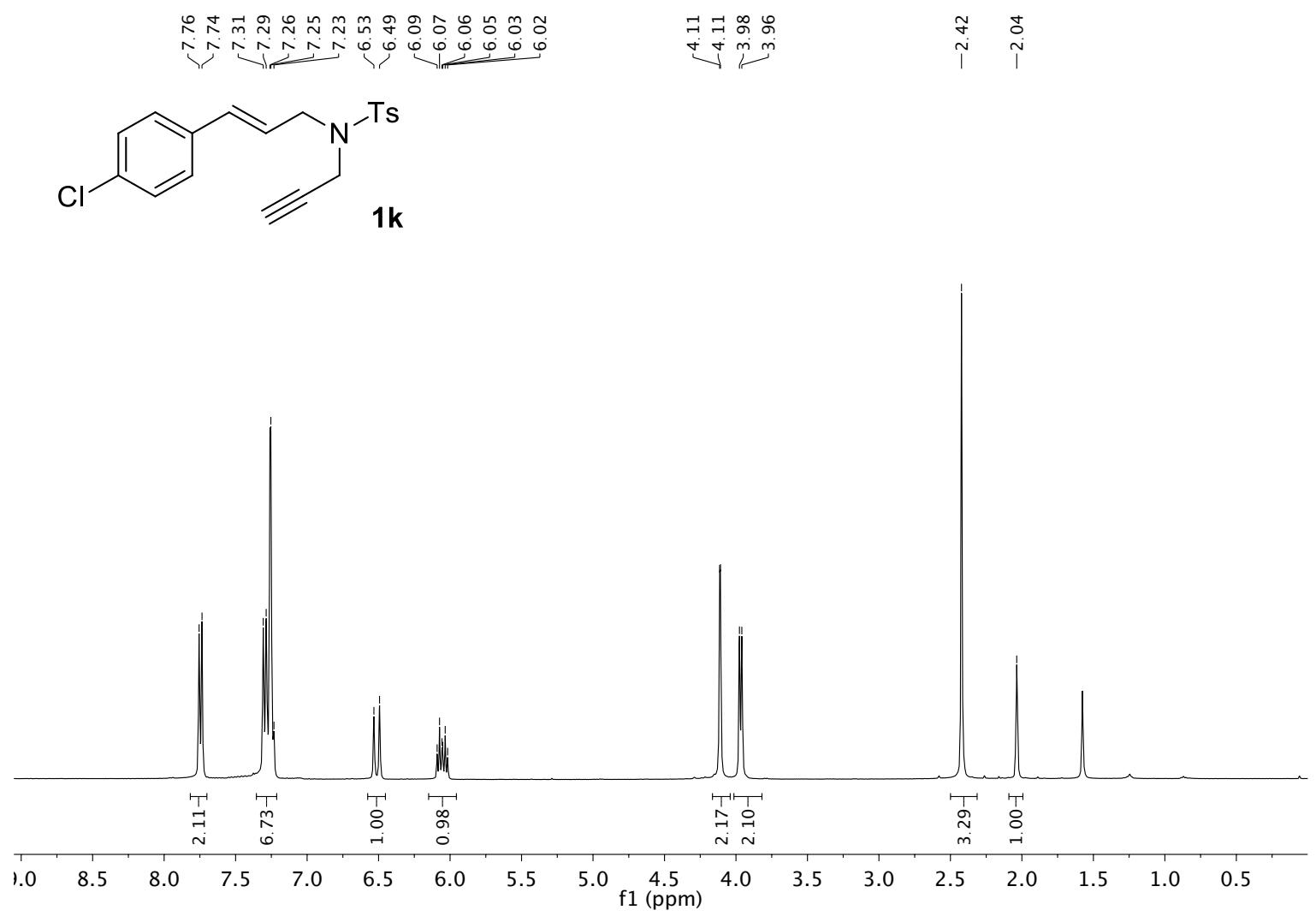
11, ${ }^{1}$ H NMR $\left(400 \mathrm{MHz}, \mathrm{CDCl}_{3}\right)$

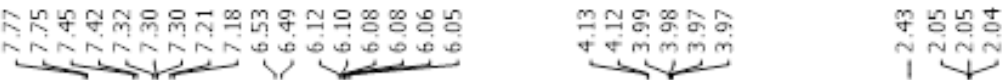<smiles>C#CCN([13CH3])C/C=C/c1ccc(Br)cc1</smiles>

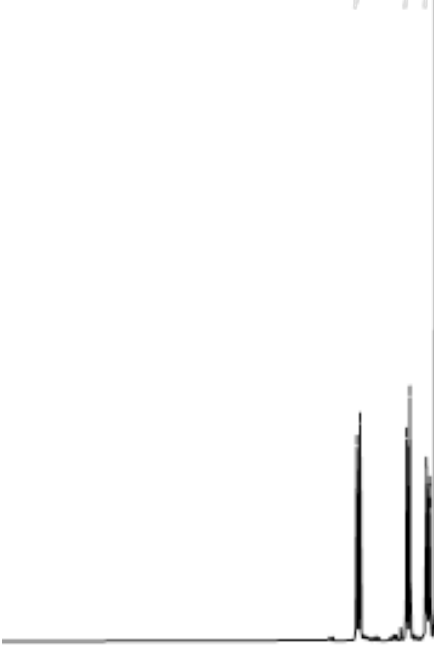

dू का क्ष
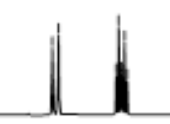

d

ds

f)

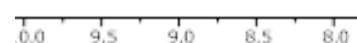

s.

11, ${ }^{13} \mathbf{C}\{\mathbf{H}\}$ NMR $\left(101 \mathrm{MHz}, \mathrm{CDCl}_{3}\right)$

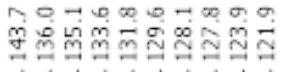

1414

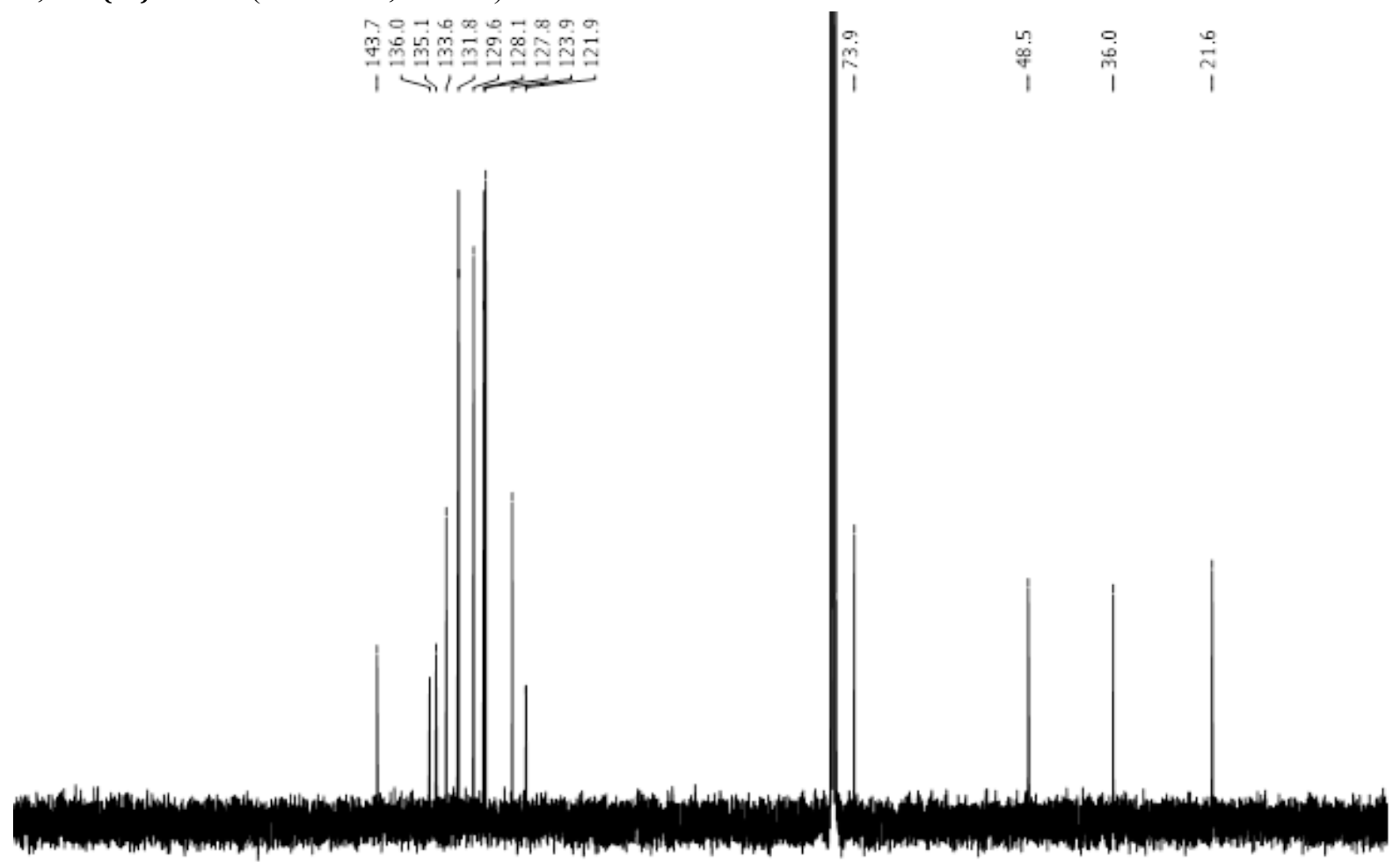

(apm)

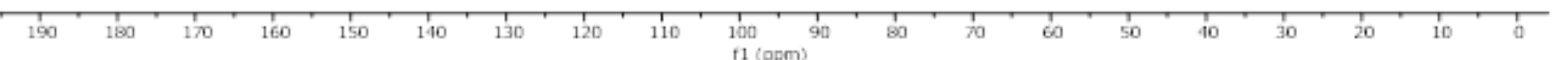


1m, ${ }^{1} \mathbf{H}$ NMR (300 MHz, $\left.\mathrm{CDCl}_{3}\right)$
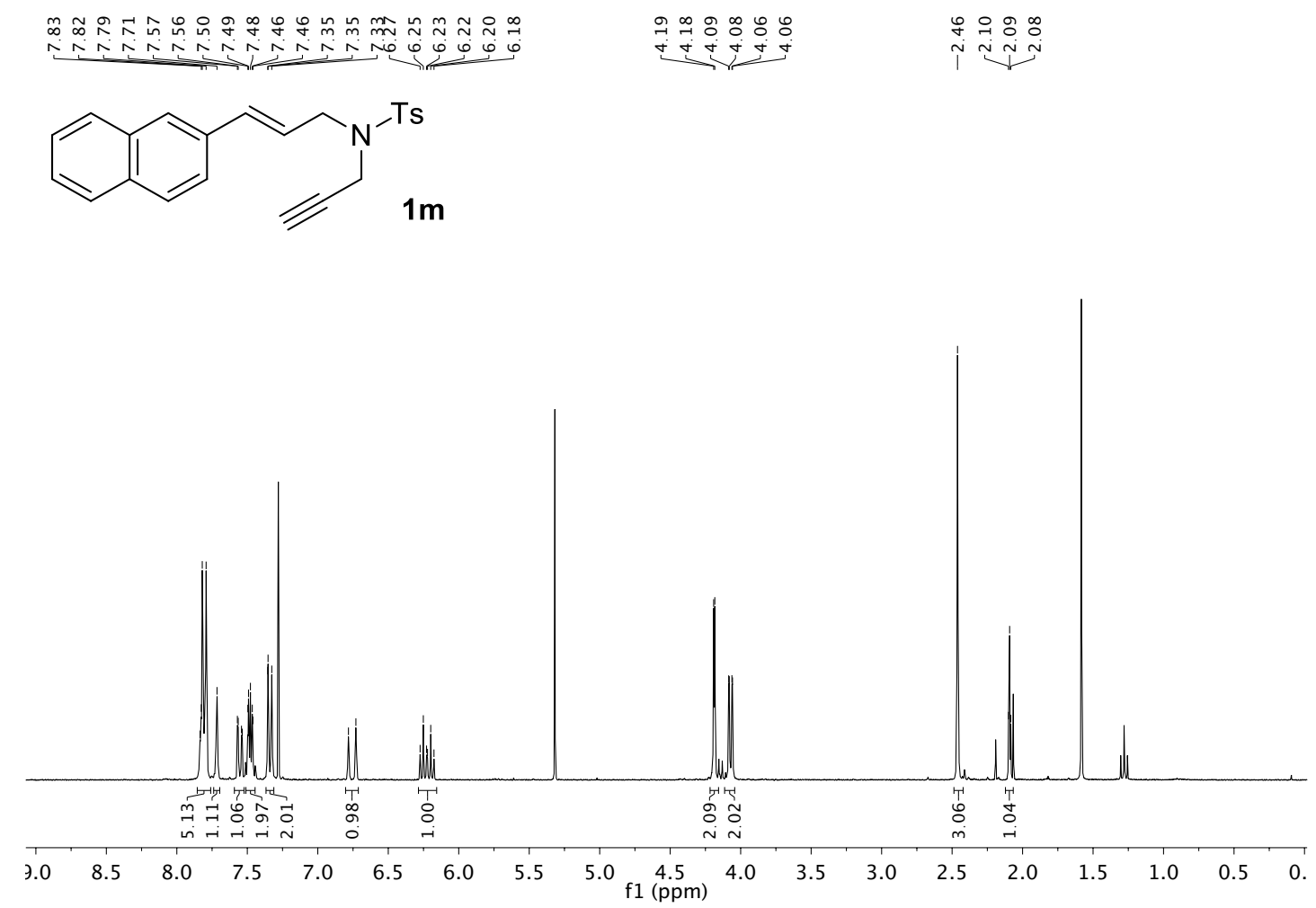

1 m, ${ }^{13} \mathbf{C}\{\mathbf{H}\} \mathbf{N M R}\left(75 \mathrm{MHz}, \mathrm{CDCl}_{3}\right)$

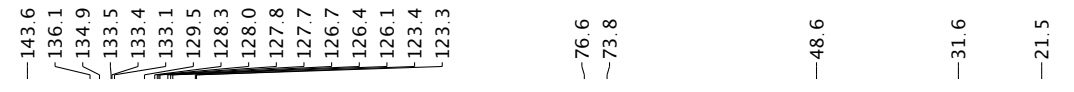

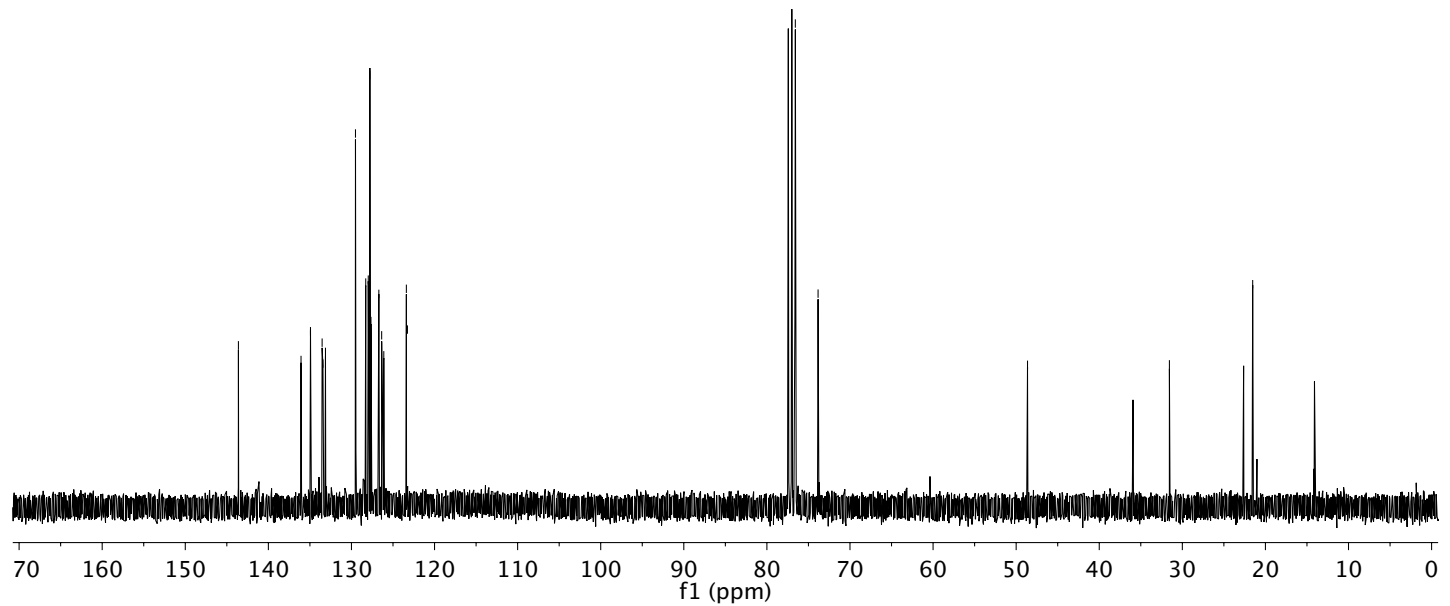


1n, ${ }^{1} \mathbf{H}$ NMR $\left(300 \mathrm{MHz}, \mathrm{CDCl}_{3}\right)$

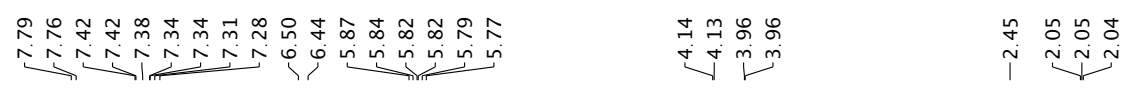<smiles>C#CCN([135I])C/C=C/c1ccoc1</smiles>

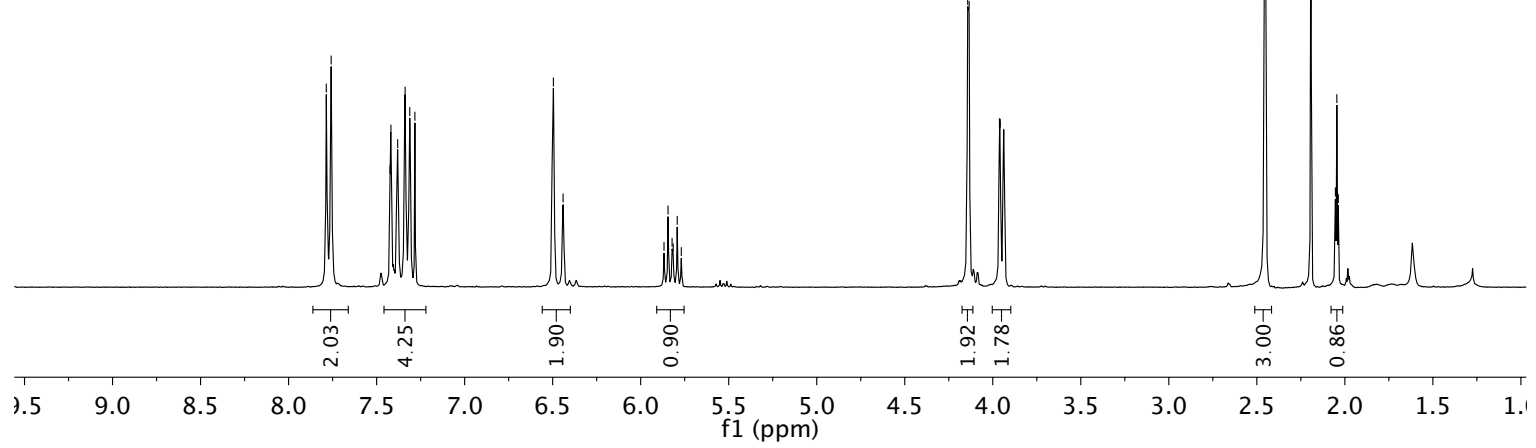


1o, ${ }^{1} \mathbf{H}$ NMR $\left(400 \mathrm{MHz}, \mathrm{CDCl}_{3}\right)$

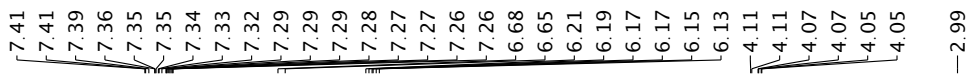<smiles>C#CCN(C)S(C)(=O)=O</smiles>

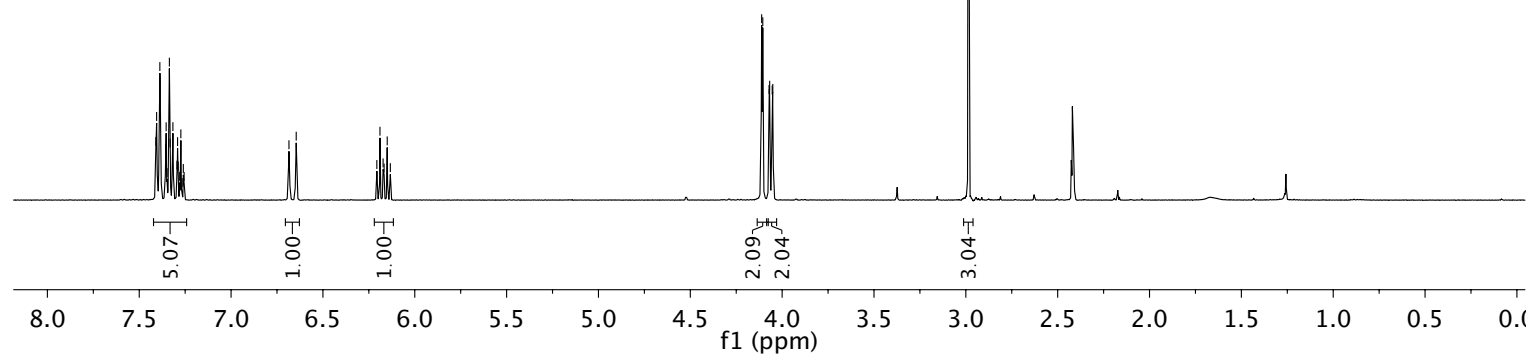

1o, ${ }^{13} \mathbf{C}\{\mathbf{H}\}$ NMR $\left(101 \mathrm{MHz}, \mathrm{CDCl}_{3}\right)$

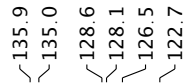

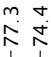

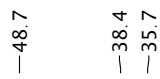

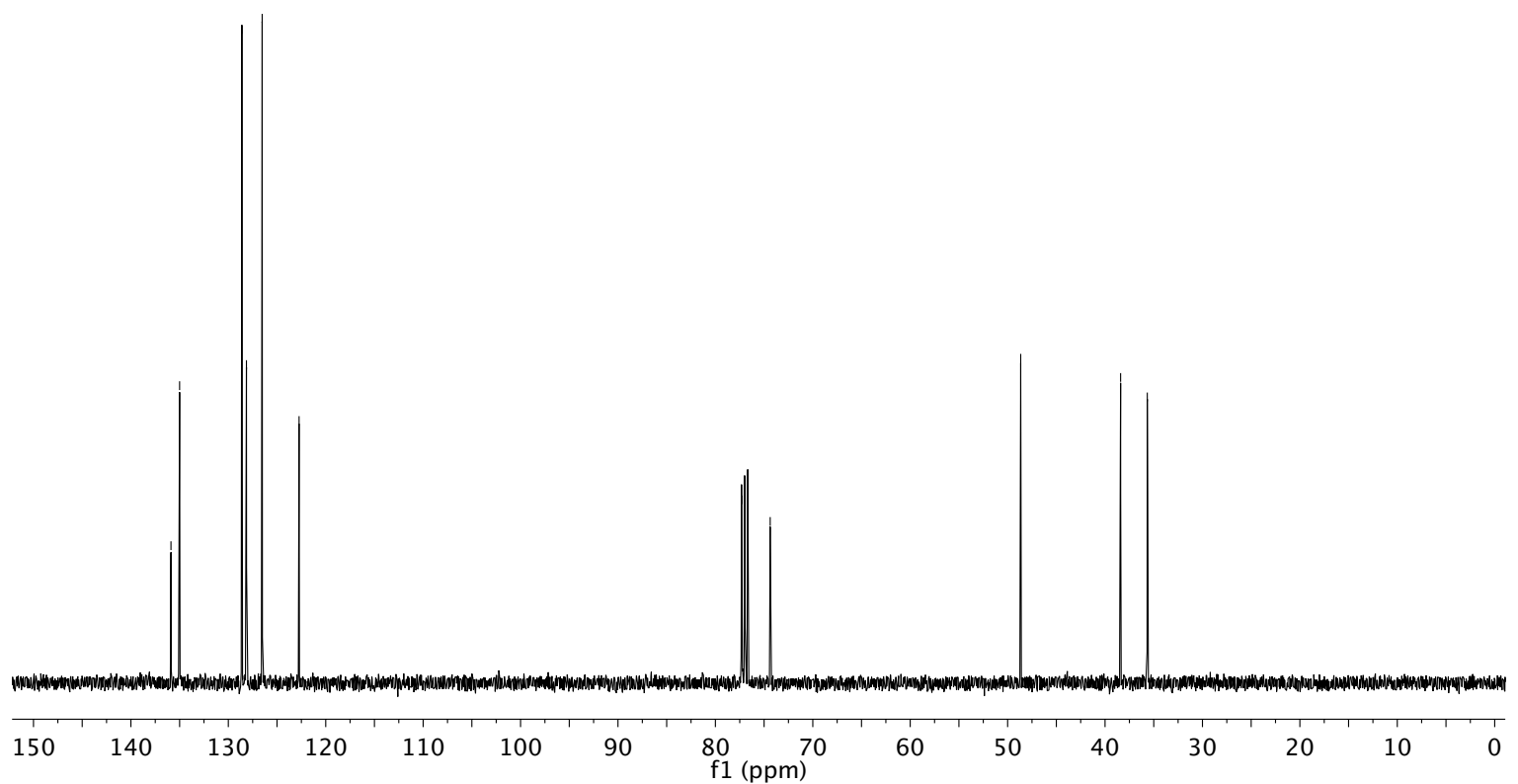


1p, ${ }^{1} \mathbf{H}$ NMR $\left(400 \mathrm{MHz}, \mathrm{CDCl}_{3}\right)$

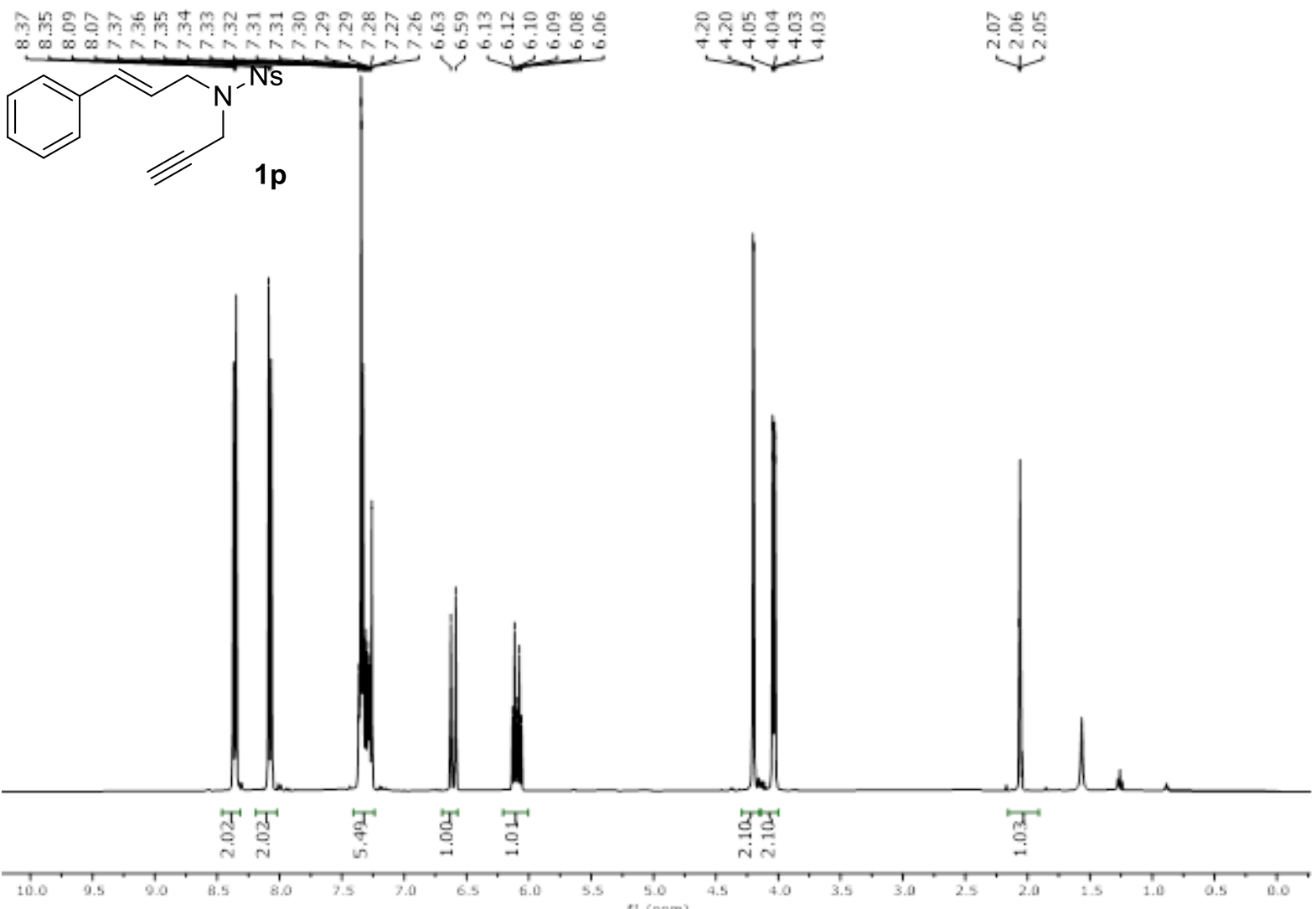

1p, ${ }^{13} \mathbf{C}\{\mathbf{H}\} \mathbf{N M R}\left(101 \mathrm{MHz}, \mathrm{CDCl}_{3}\right)$

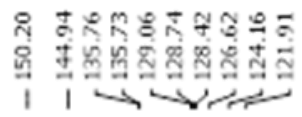

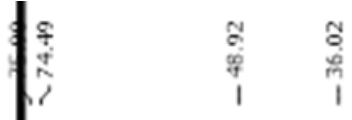
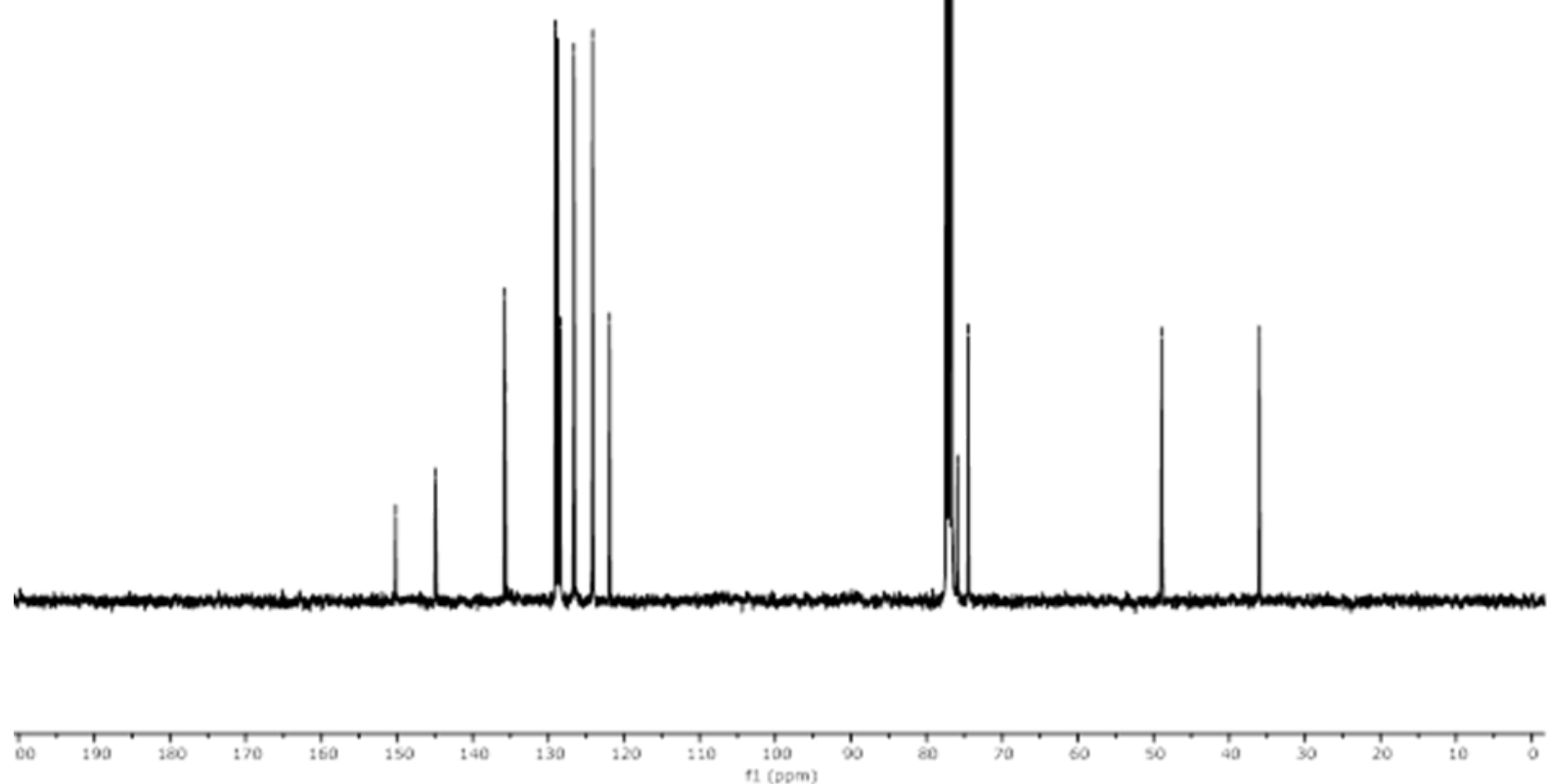
1q, ${ }^{1} \mathbf{H}$ NMR $\left(400 \mathrm{MHz}, \mathrm{CDCl}_{3}\right)$

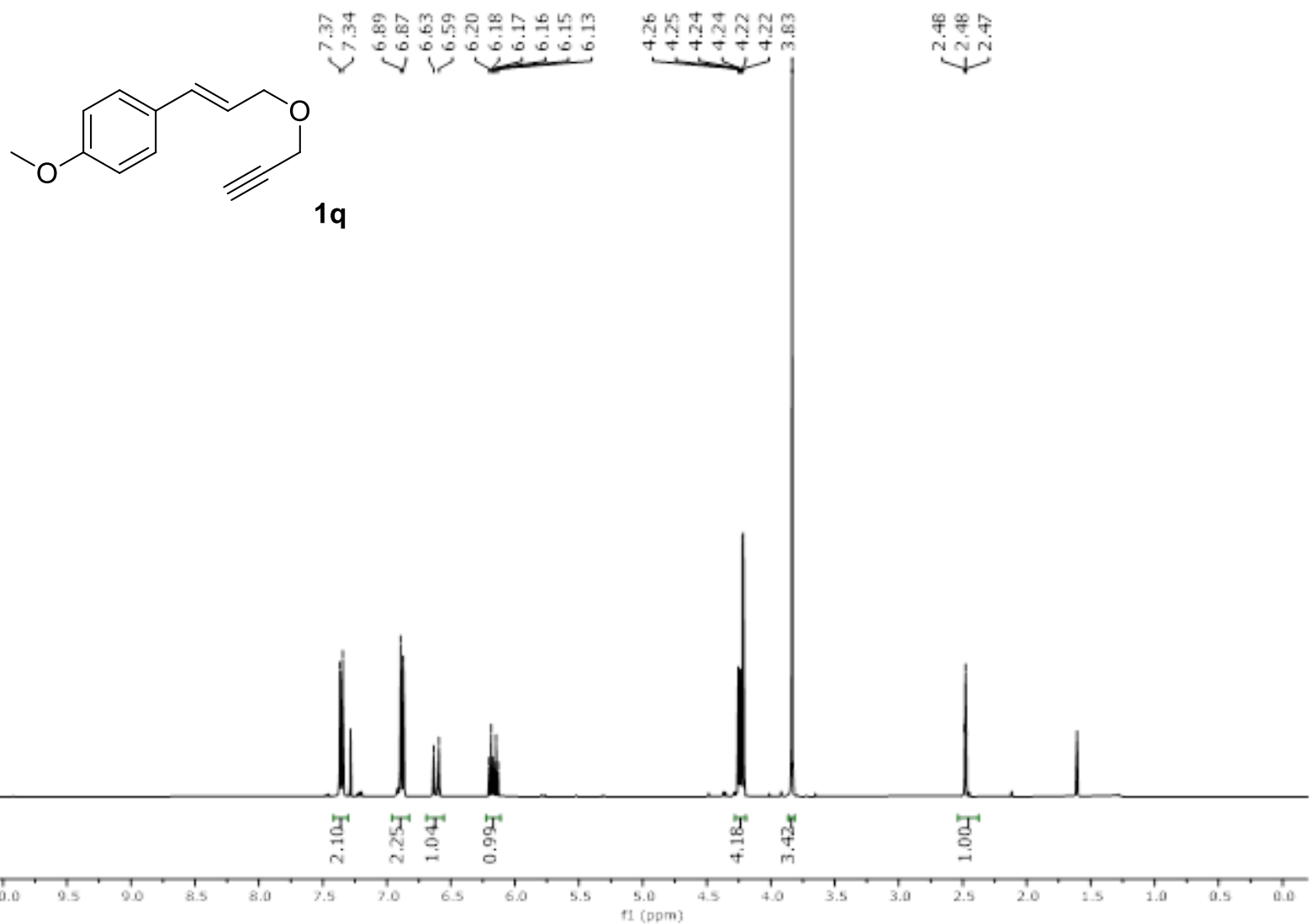

1q, ${ }^{13} \mathbf{C}\{\mathbf{H}\}$ NMR $\left(101 \mathrm{MHz}, \mathrm{CDCl}_{3}\right)$

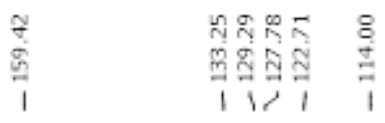

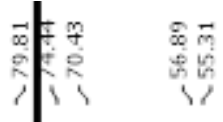
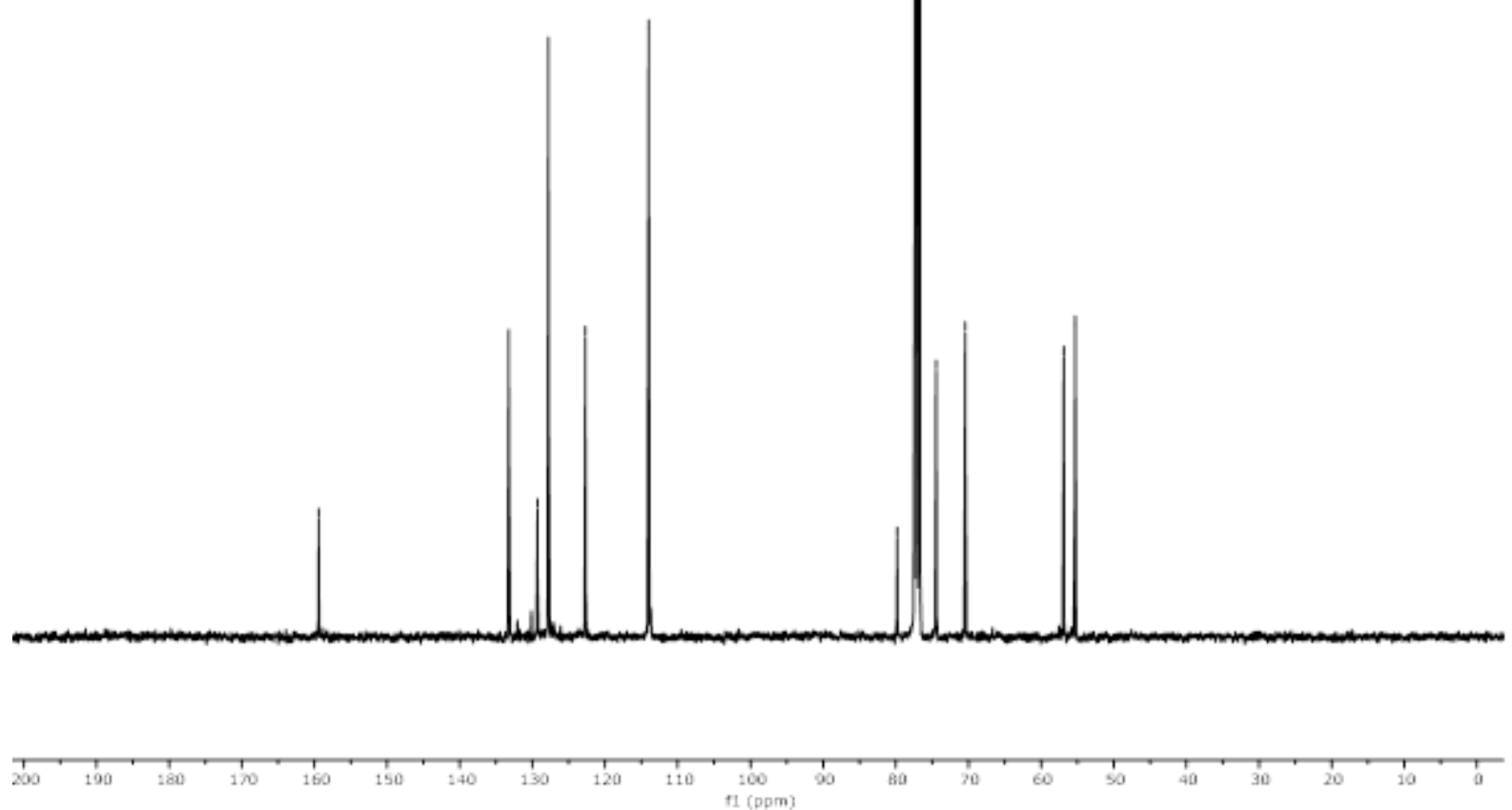
1r, ${ }^{1} \mathbf{H}$ NMR $\left(400 \mathrm{MHz}, \mathrm{CDCl}_{3}\right)$
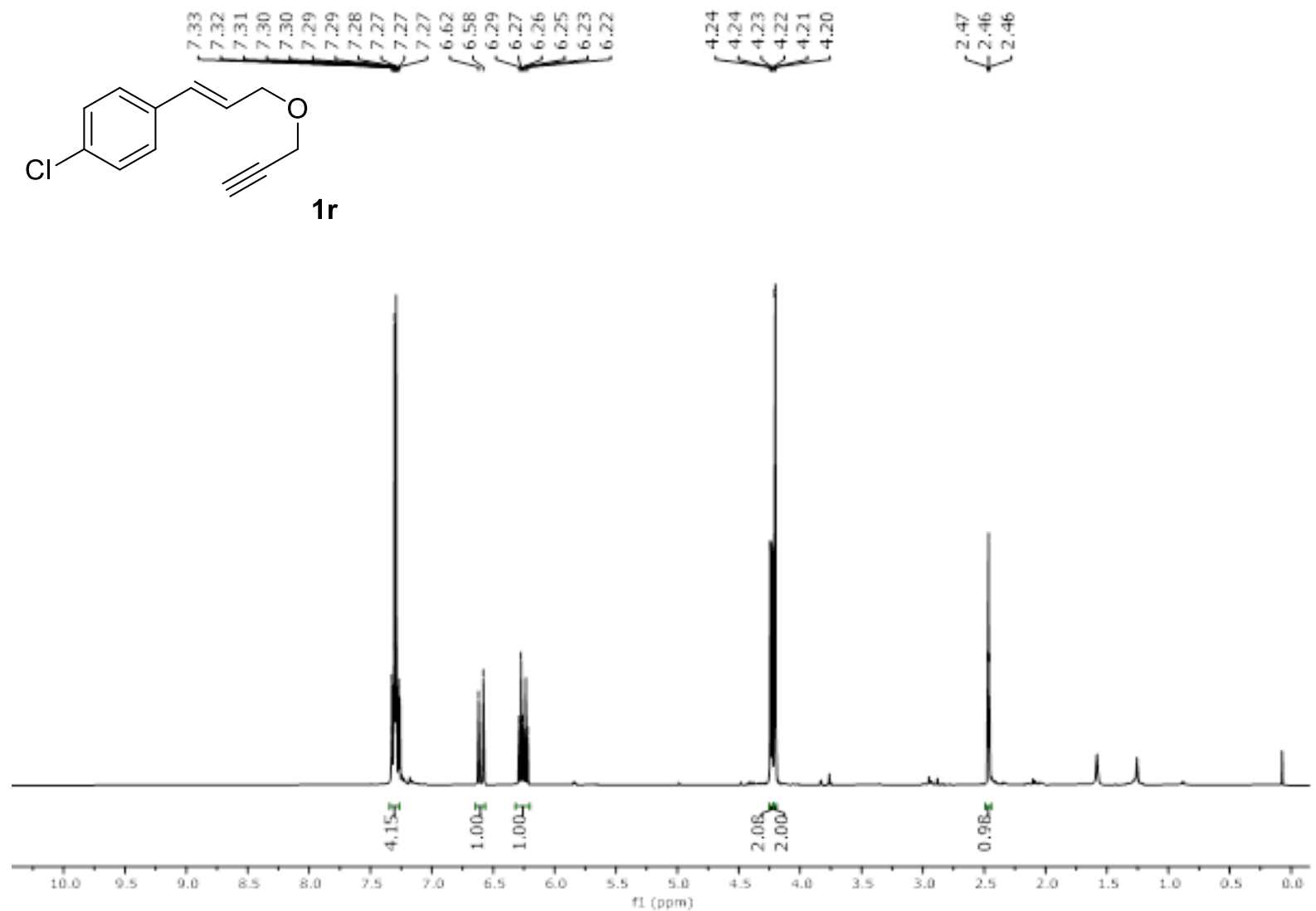

1r, ${ }^{13} \mathbf{C}\{\mathbf{H}\}$ NMR $\left(101 \mathrm{MHz}, \mathrm{CDCl}_{3}\right)$
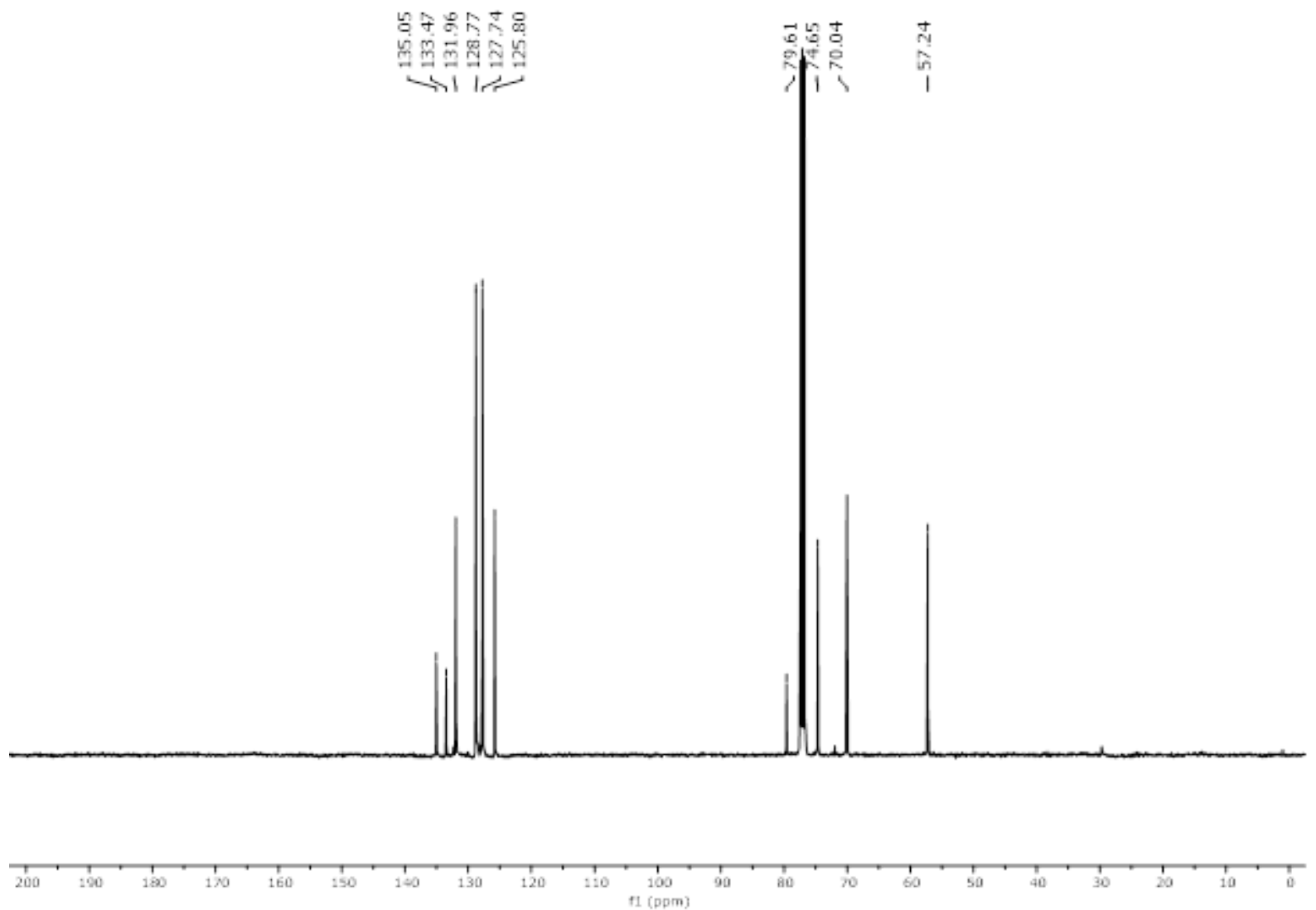
1s, ${ }^{1} \mathbf{H}$ NMR $\left(400 \mathrm{MHz}, \mathrm{CDCl}_{3}\right)$

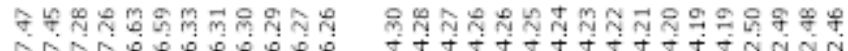

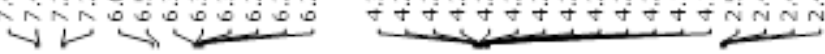<smiles>C#CCOC/C=C/c1ccc(Br)cc1</smiles>

$1 \mathrm{~s}$

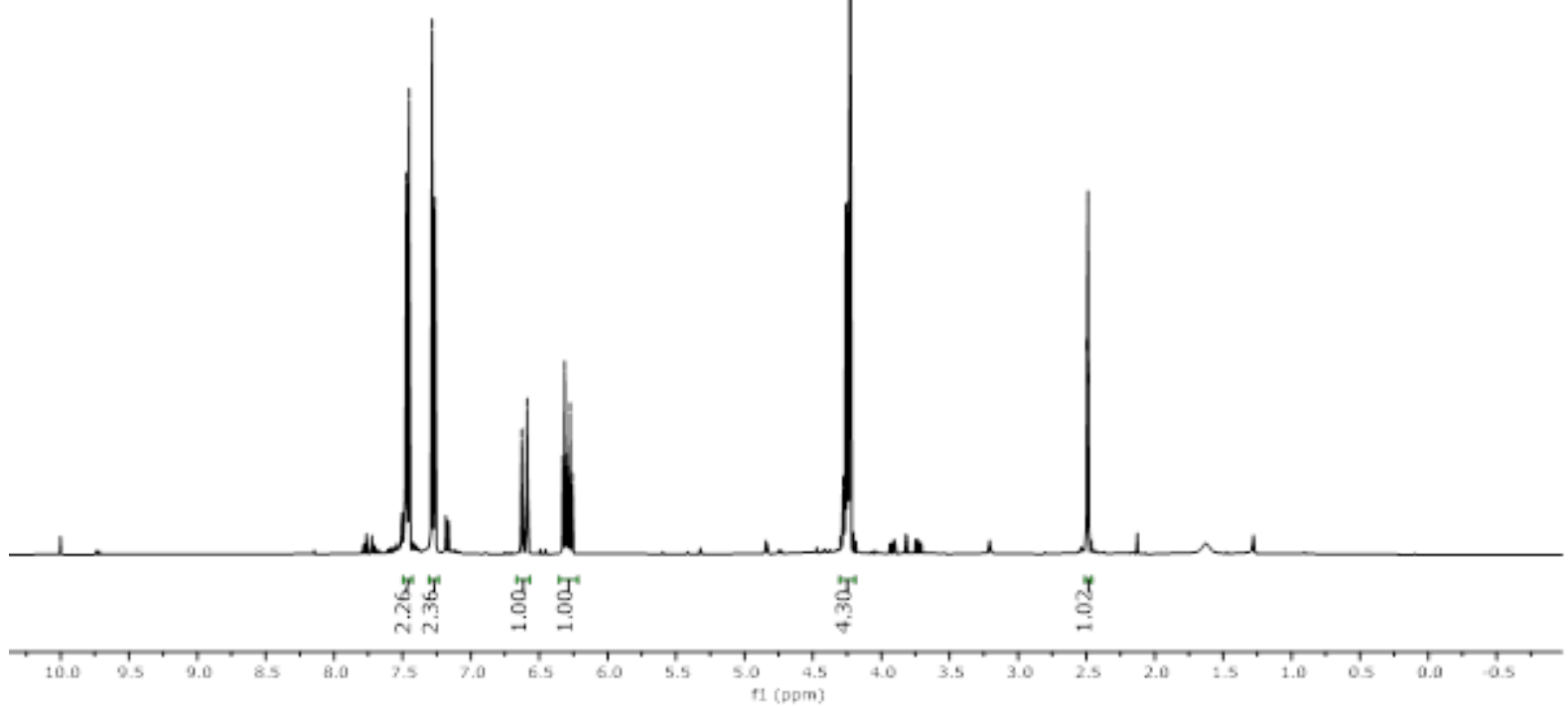

1s, ${ }^{13} \mathbf{C}\{\mathbf{H}\}$ NMR $\left(101 \mathrm{MHz}, \mathrm{CDCl}_{3}\right)$

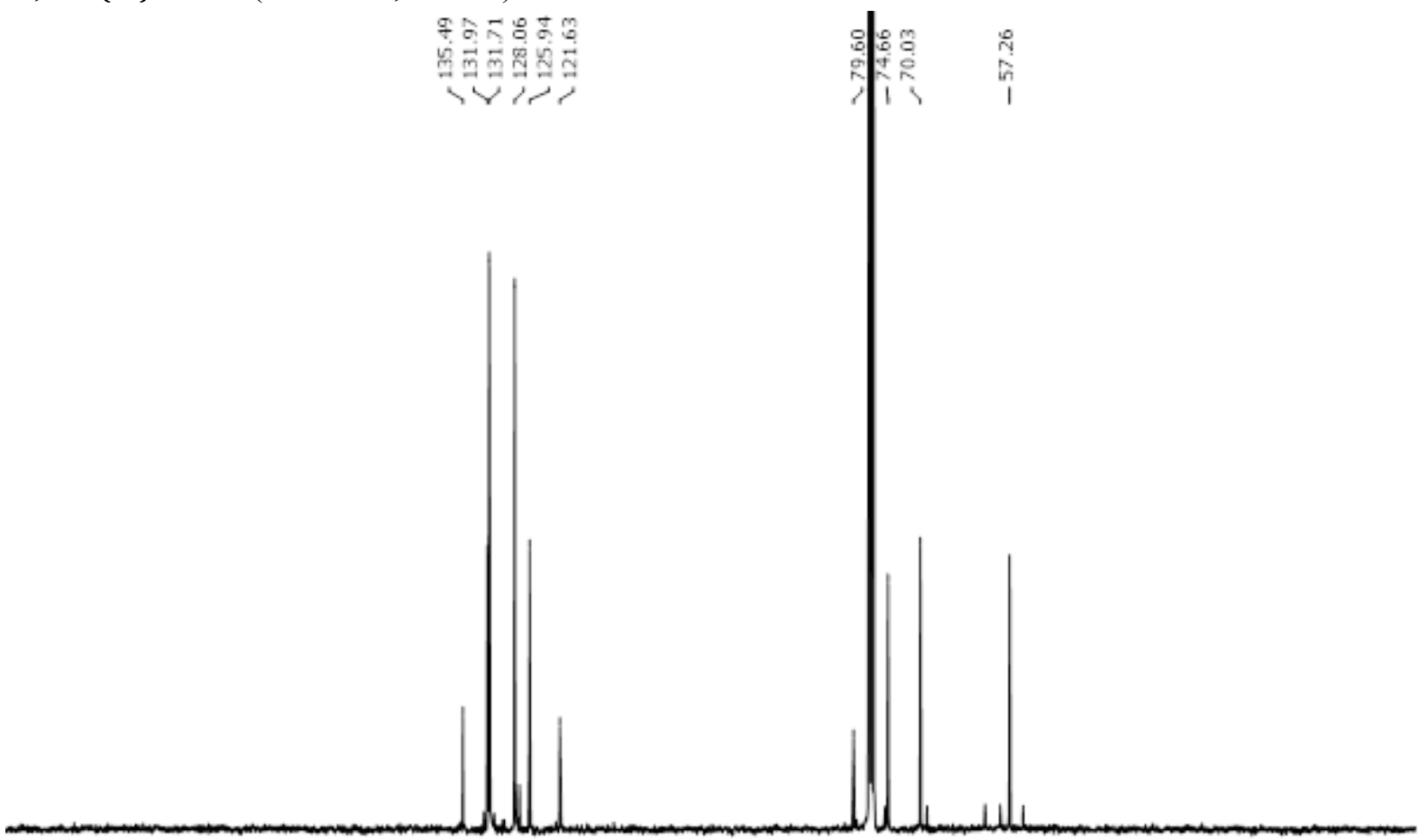


1t, ${ }^{1} \mathbf{H}$ NMR $\left(400 \mathrm{MHz}, \mathrm{CDCl}_{3}\right)$

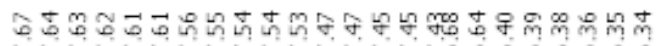

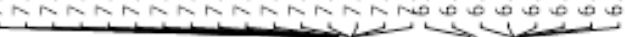

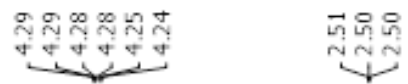

$\overbrace{\mathrm{CN}}$

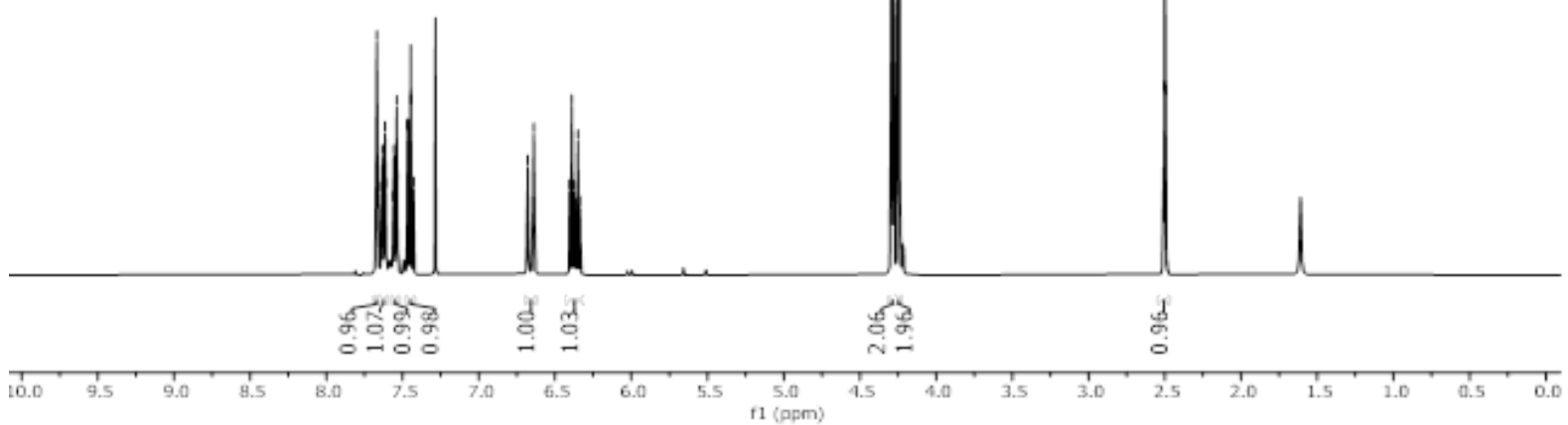

1t, ${ }^{13} \mathbf{C}\{\mathbf{H}\}$ NMR $\left(101 \mathrm{MHz}, \mathrm{CDCl}_{3}\right)$

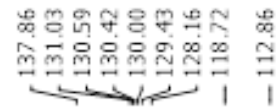

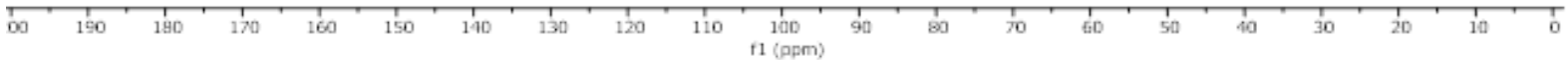


1u, ${ }^{1} \mathbf{H}$ NMR $\left(400 \mathrm{MHz}, \mathrm{CDCl}_{3}\right)$

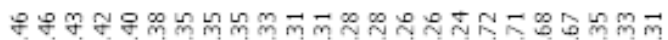

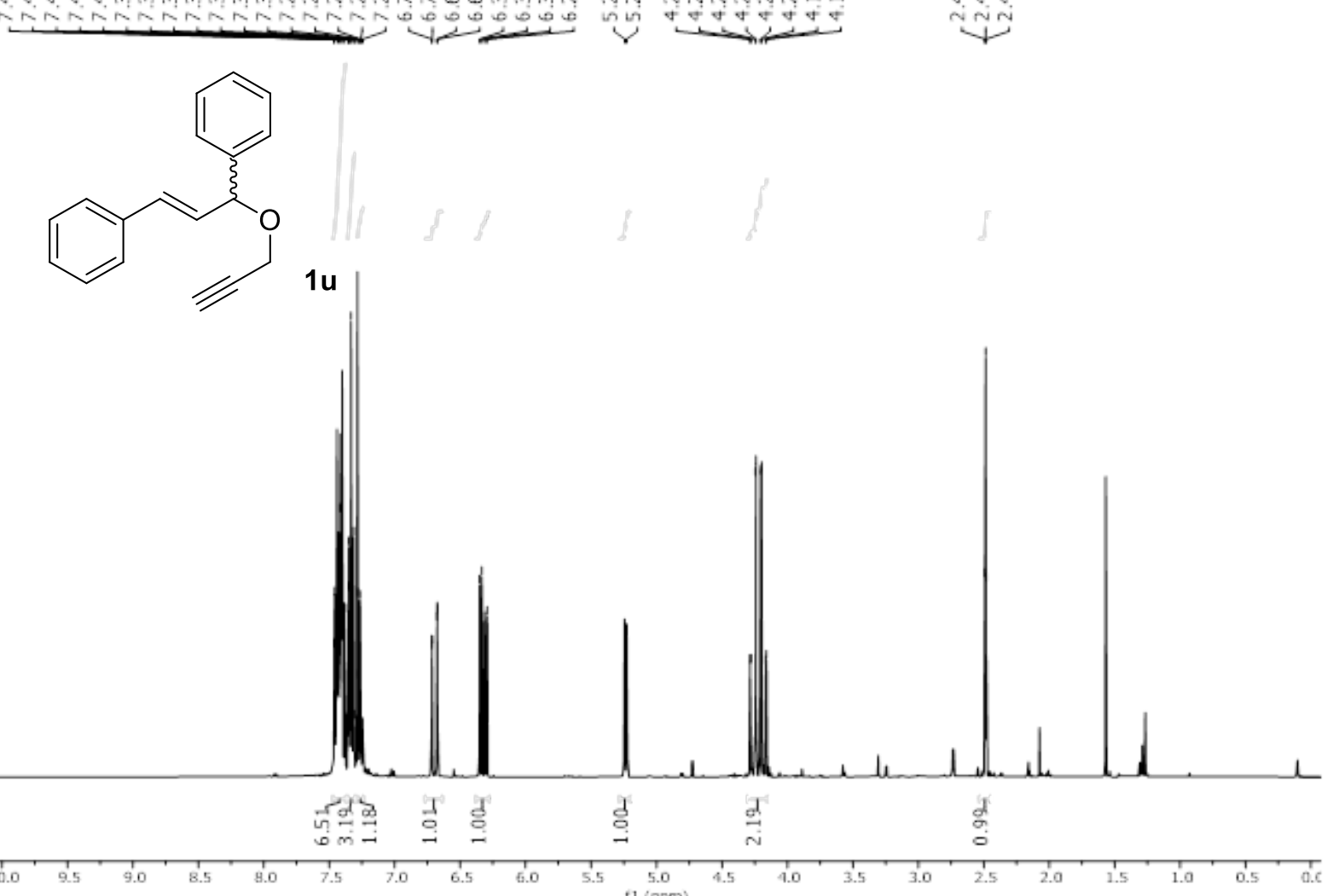

$\mathbf{1 u},{ }^{13} \mathbf{C}\{\mathbf{H}\}$ NMR $\left(101 \mathrm{MHz}, \mathrm{CDCl}_{3}\right)$

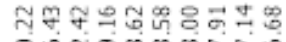

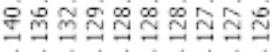

造品最

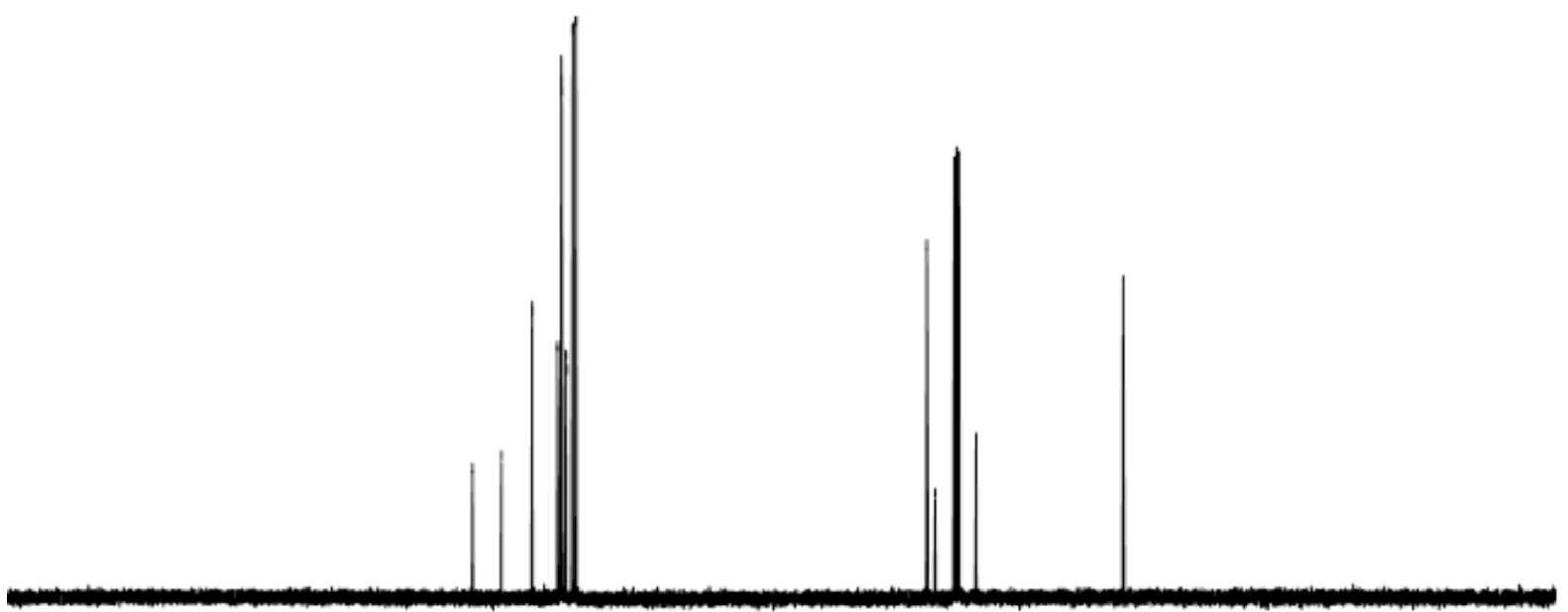

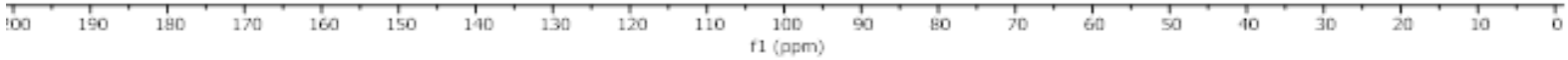


1v, ${ }^{1} \mathbf{H}$ NMR (400 MHz, $\mathrm{CDCl}_{3}$ )

ติ

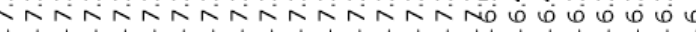

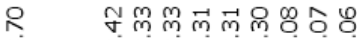



ri ni

=

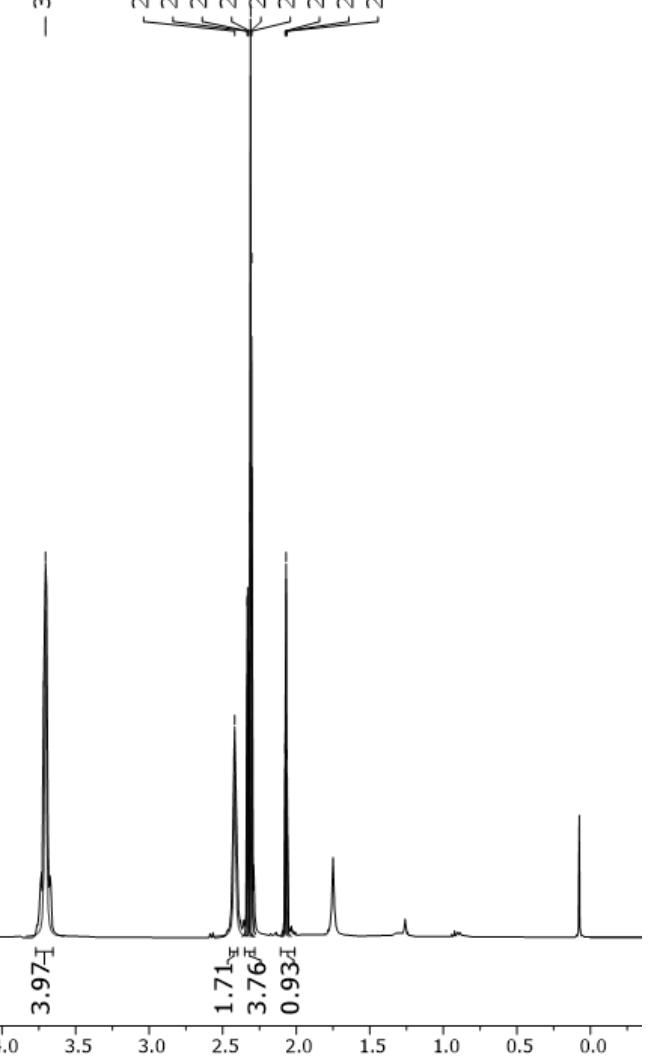


3a, ${ }^{1} \mathbf{H}$ NMR $\left(400 \mathrm{MHz}, \mathrm{CDCl}_{3}\right)$

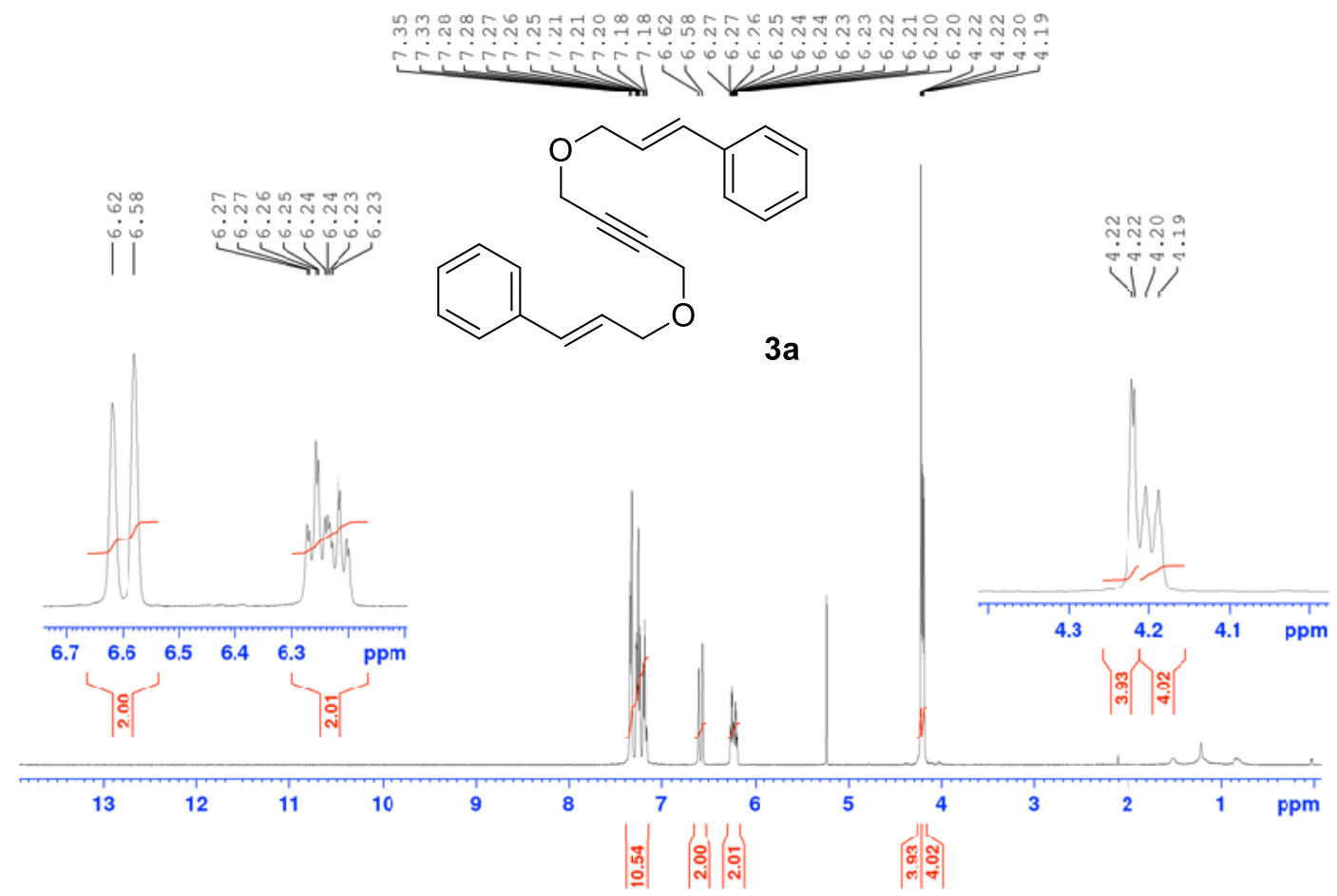


3b, ${ }^{1} \mathbf{H}$ NMR $\left(400 \mathrm{MHz}, \mathrm{CDCl}_{3}\right)$

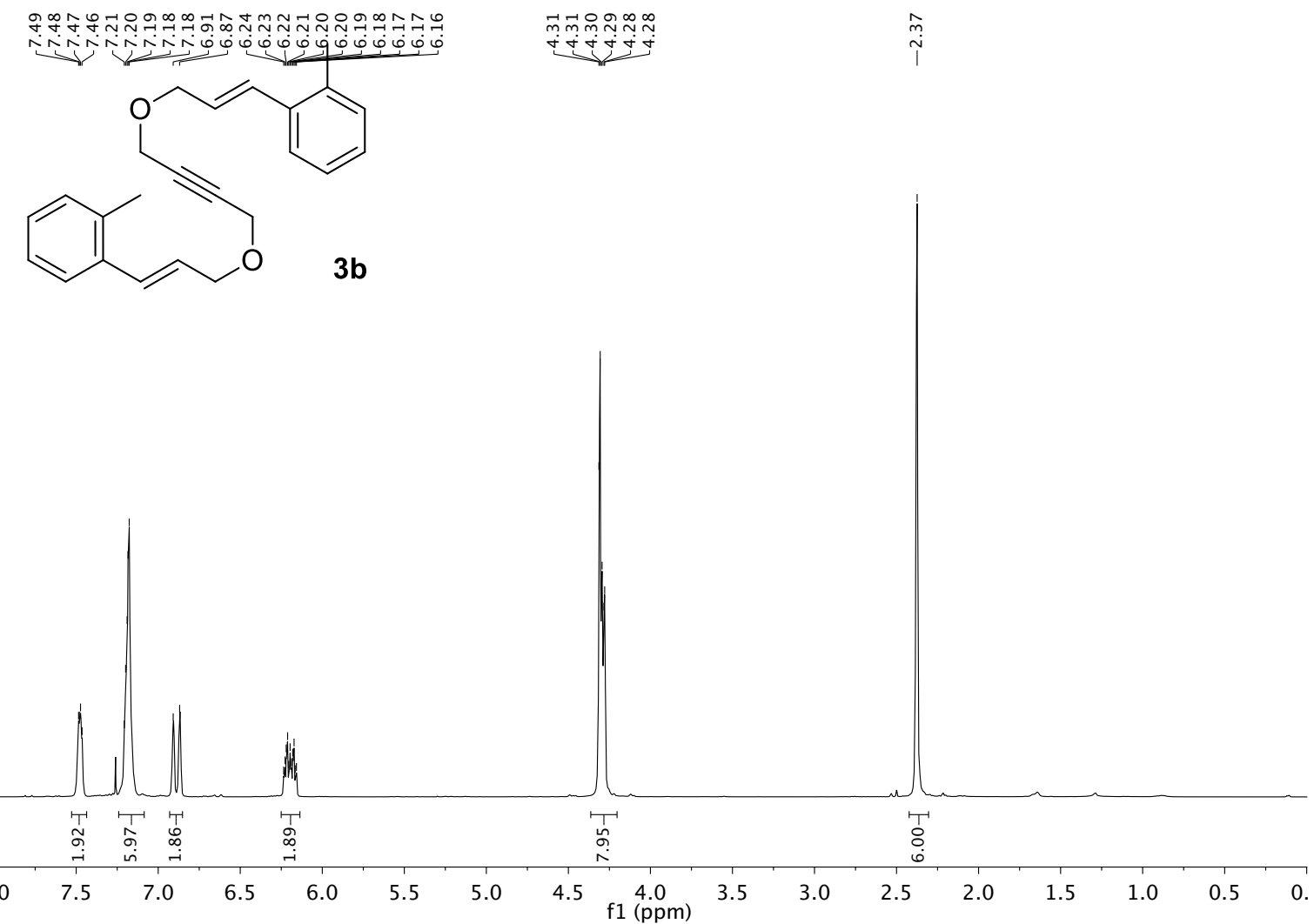


3c, ${ }^{1} \mathbf{H}$ NMR $\left(400 \mathrm{MHz}, \mathrm{CDCl}_{3}\right)$

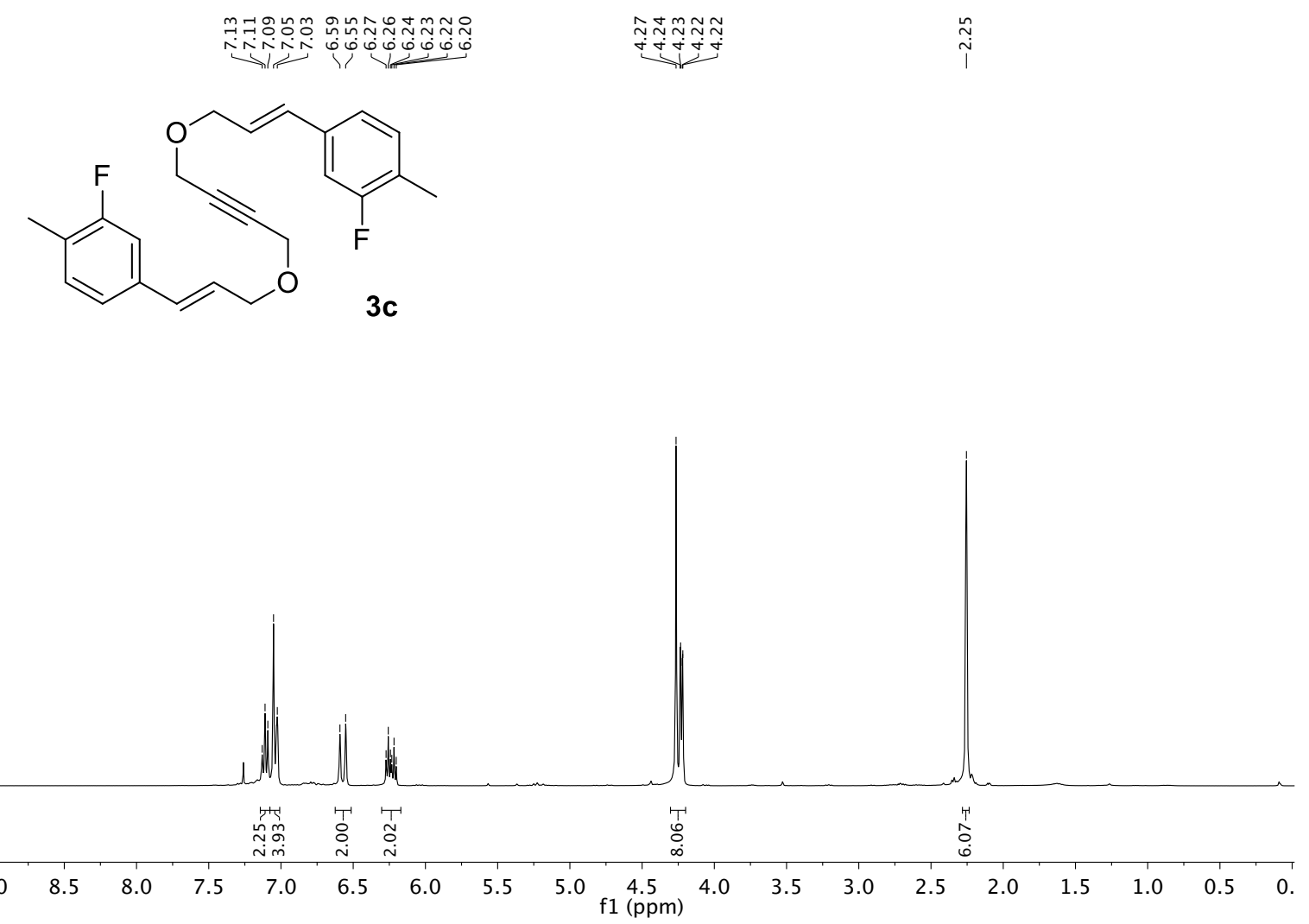


3d, ${ }^{1} \mathbf{H}$ NMR $\left(400 \mathrm{MHz}, \mathrm{CDCl}_{3}\right)$

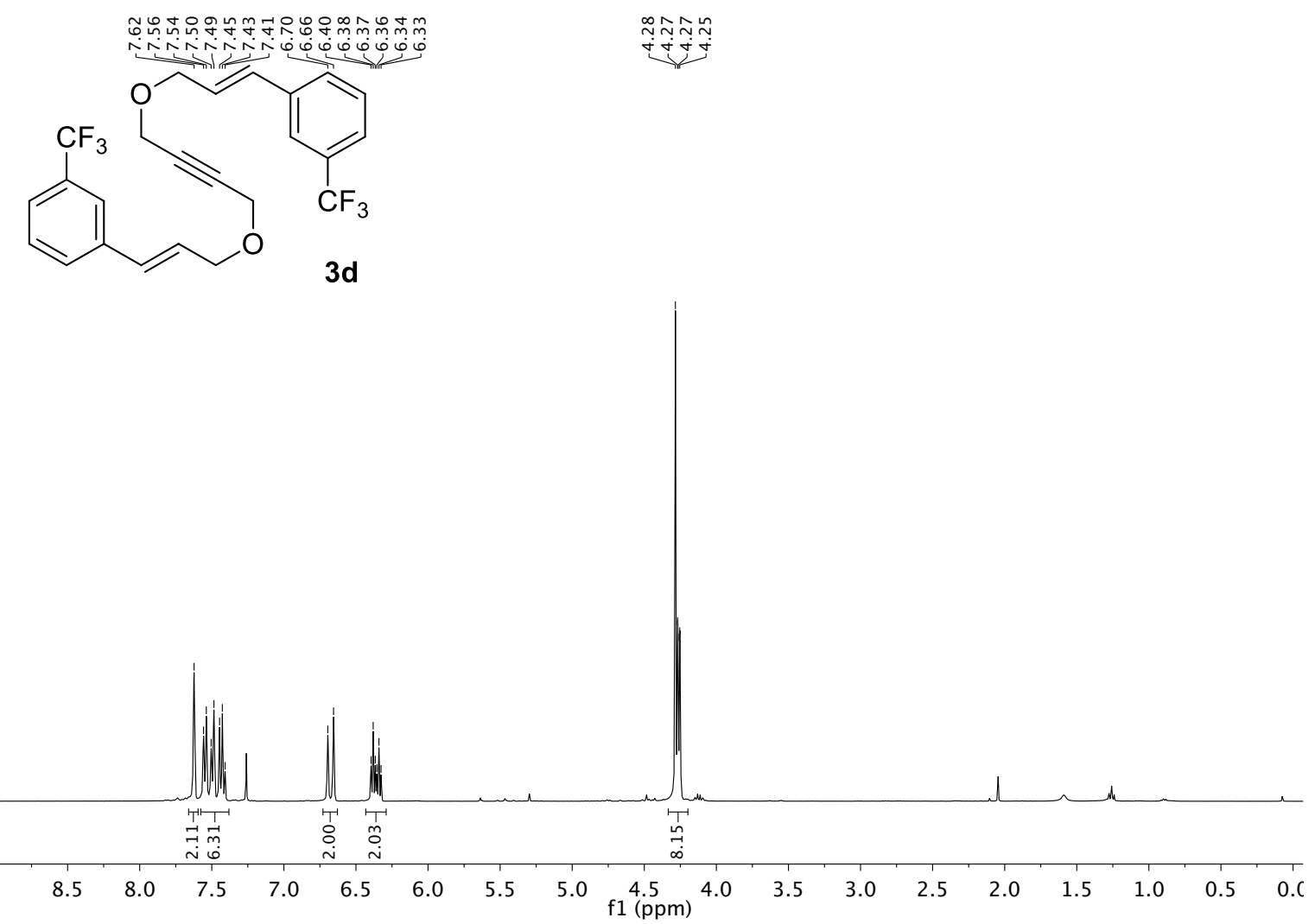


3e, ${ }^{1} \mathbf{H}$ NMR $\left(400 \mathrm{MHz}, \mathrm{CDCl}_{3}\right)$
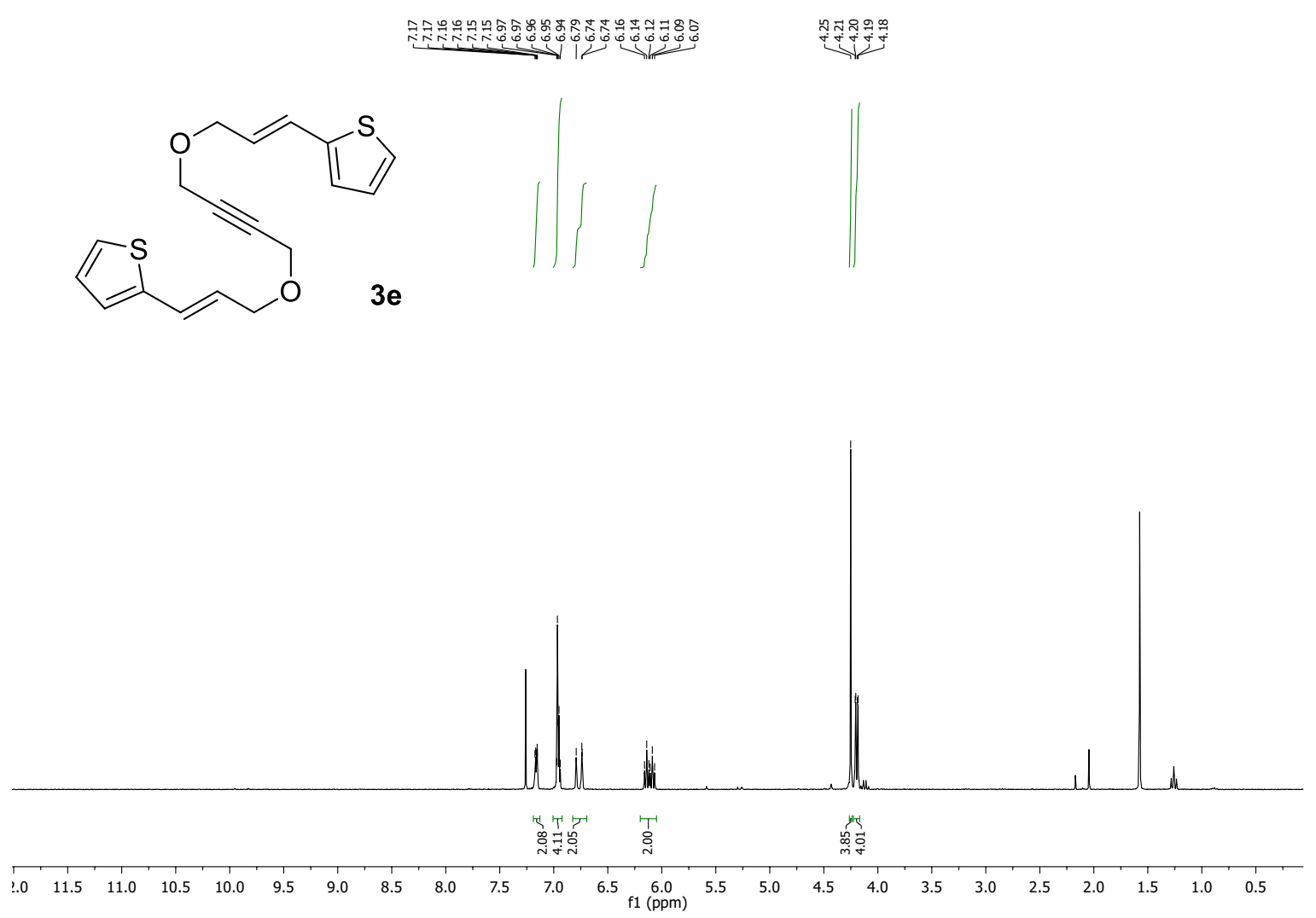
3f, ${ }^{1} \mathbf{H}$ NMR $\left(400 \mathrm{MHz}, \mathrm{CDCl}_{3}\right)$

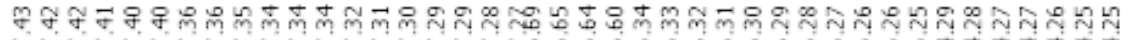

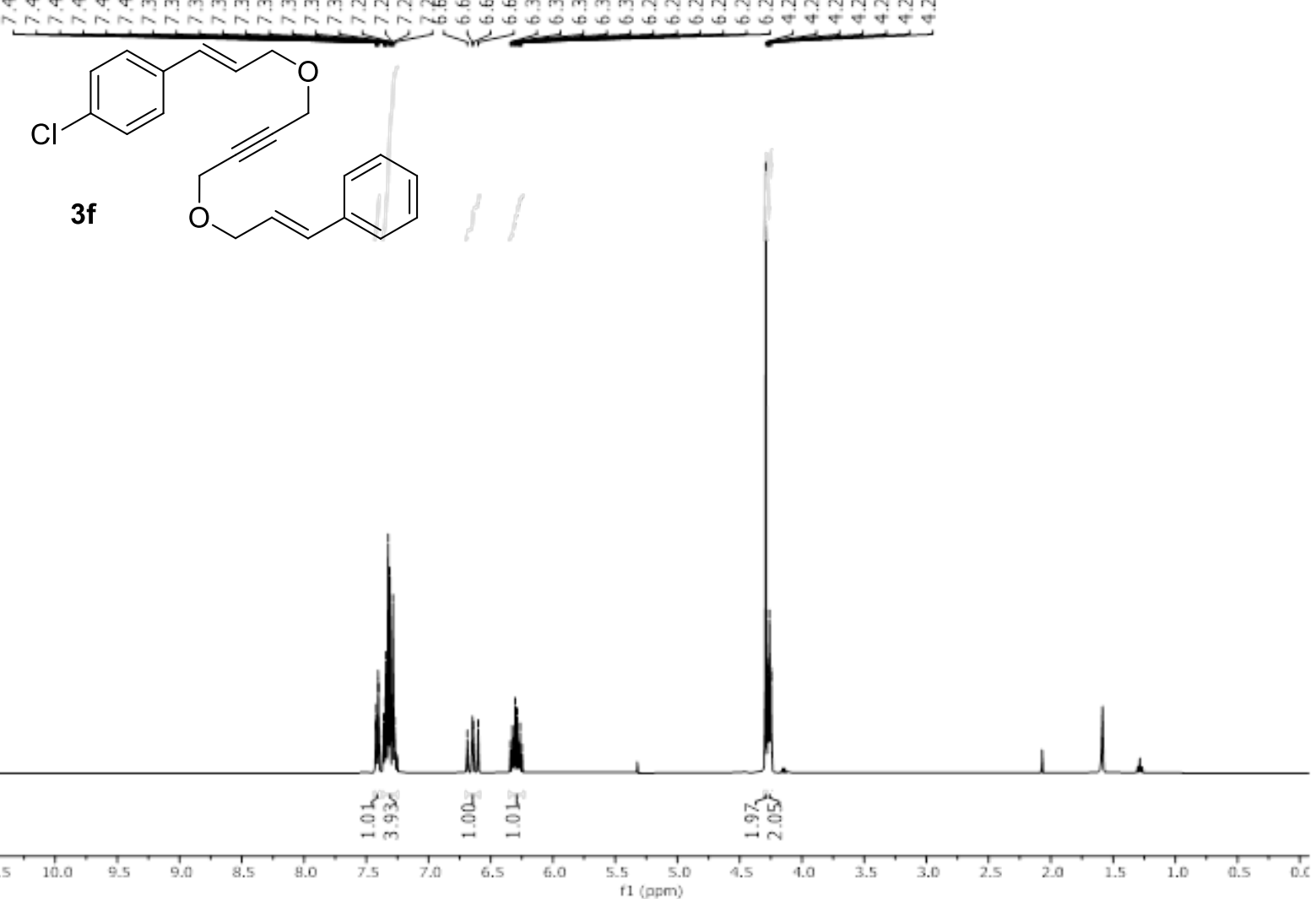

3f, ${ }^{13} \mathbf{C}\{\mathbf{H}\}$ NMR $\left(101 \mathrm{MHz}, \mathrm{CDCl}_{3}\right)$

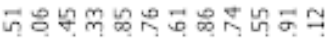

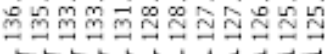

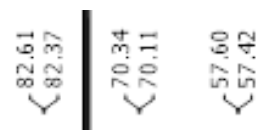

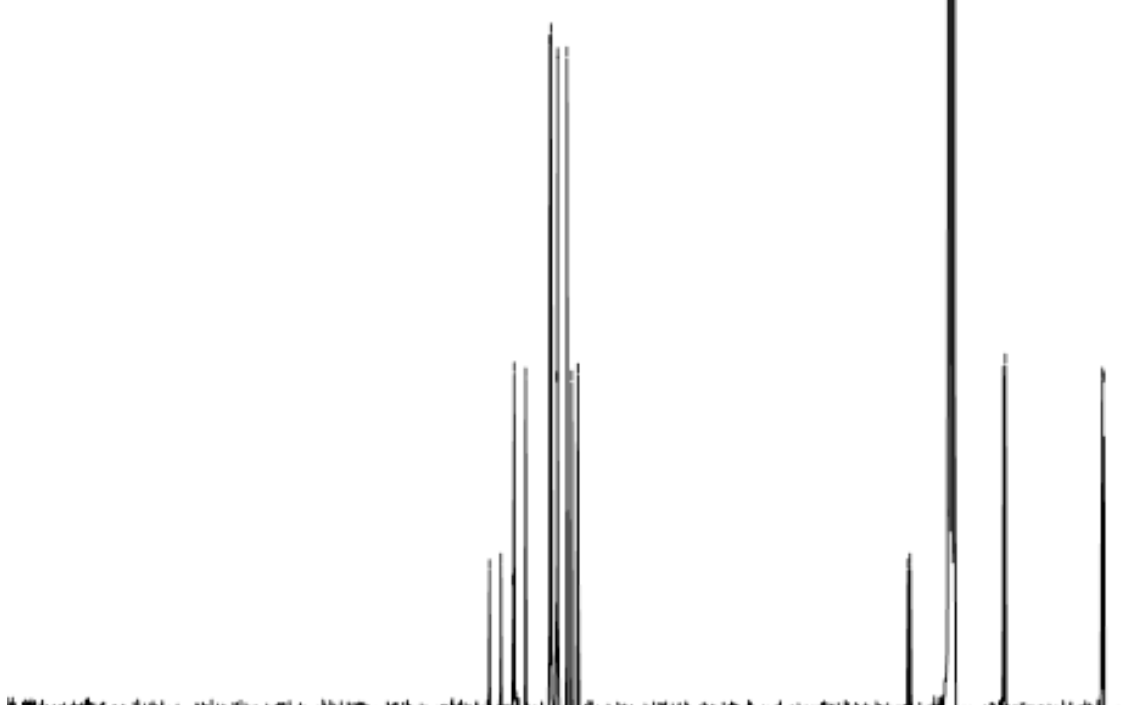

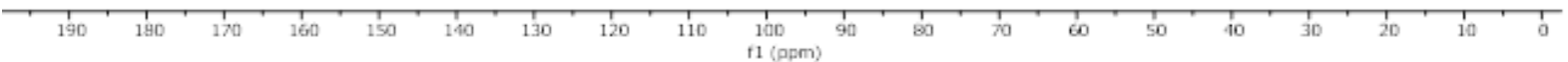


3g, ${ }^{1} \mathbf{H}$ NMR $\left(400 \mathrm{MHz}, \mathrm{CDCl}_{3}\right)$

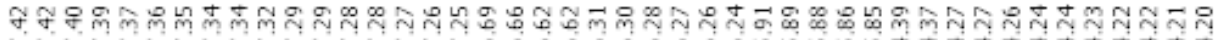

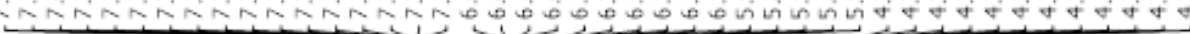

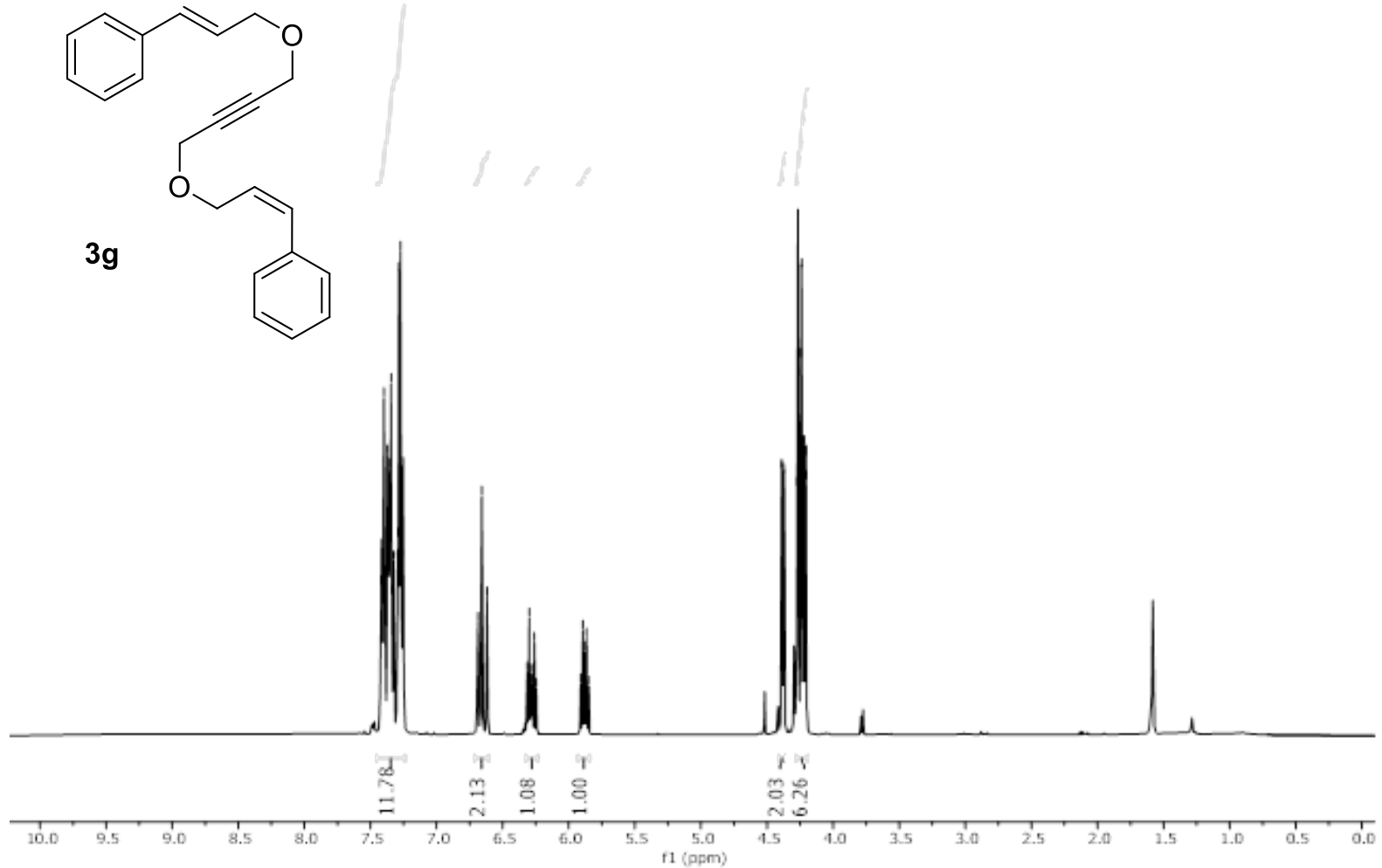

3g, ${ }^{13} \mathbf{C}\{\mathbf{H}\}$ NMR $\left(101 \mathrm{MHz}, \mathrm{CDCl}_{3}\right)$

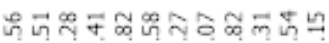

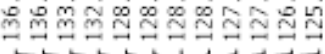

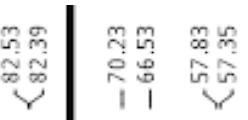

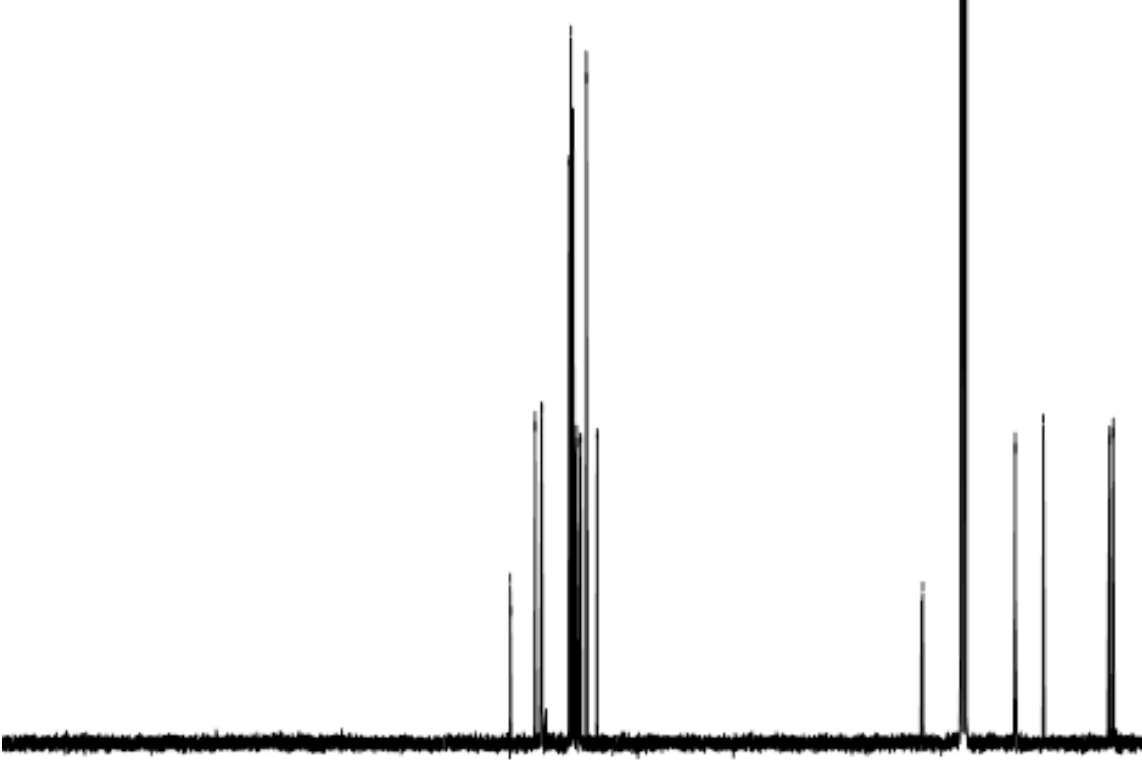

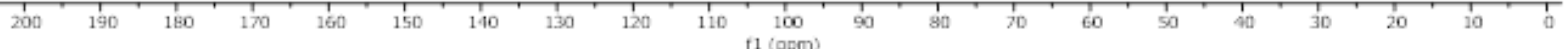


3a, ${ }^{1} \mathbf{H}$ NMR $\left(300 \mathrm{MHz}, \mathrm{CDCl}_{3}\right)$
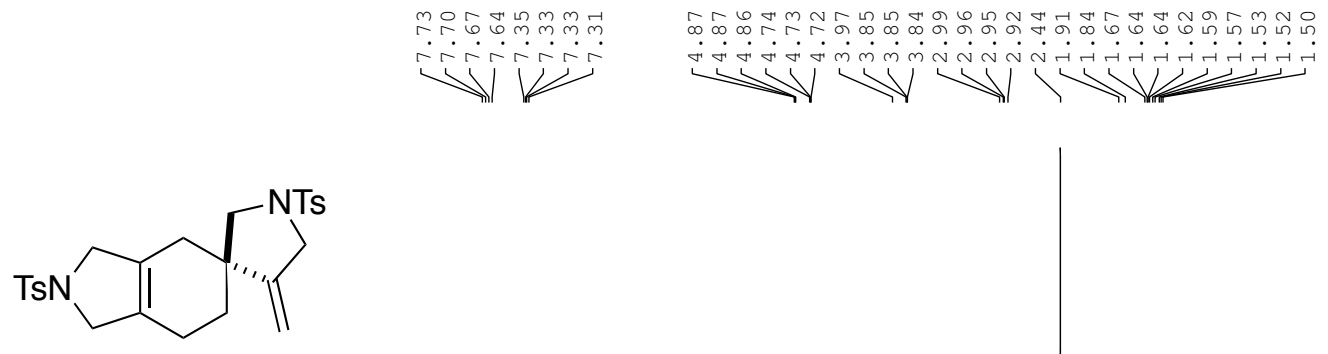

2a

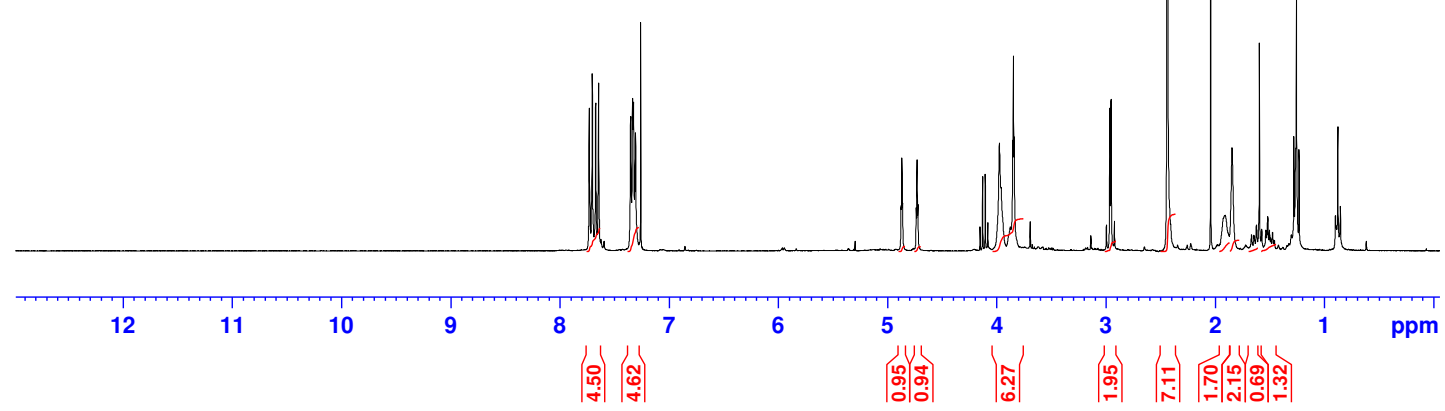

2a, ${ }^{13} \mathbf{C}\{\mathbf{H}\}$ NMR $\left(75 \mathrm{MHz}, \mathrm{CDCl}_{3}\right)$

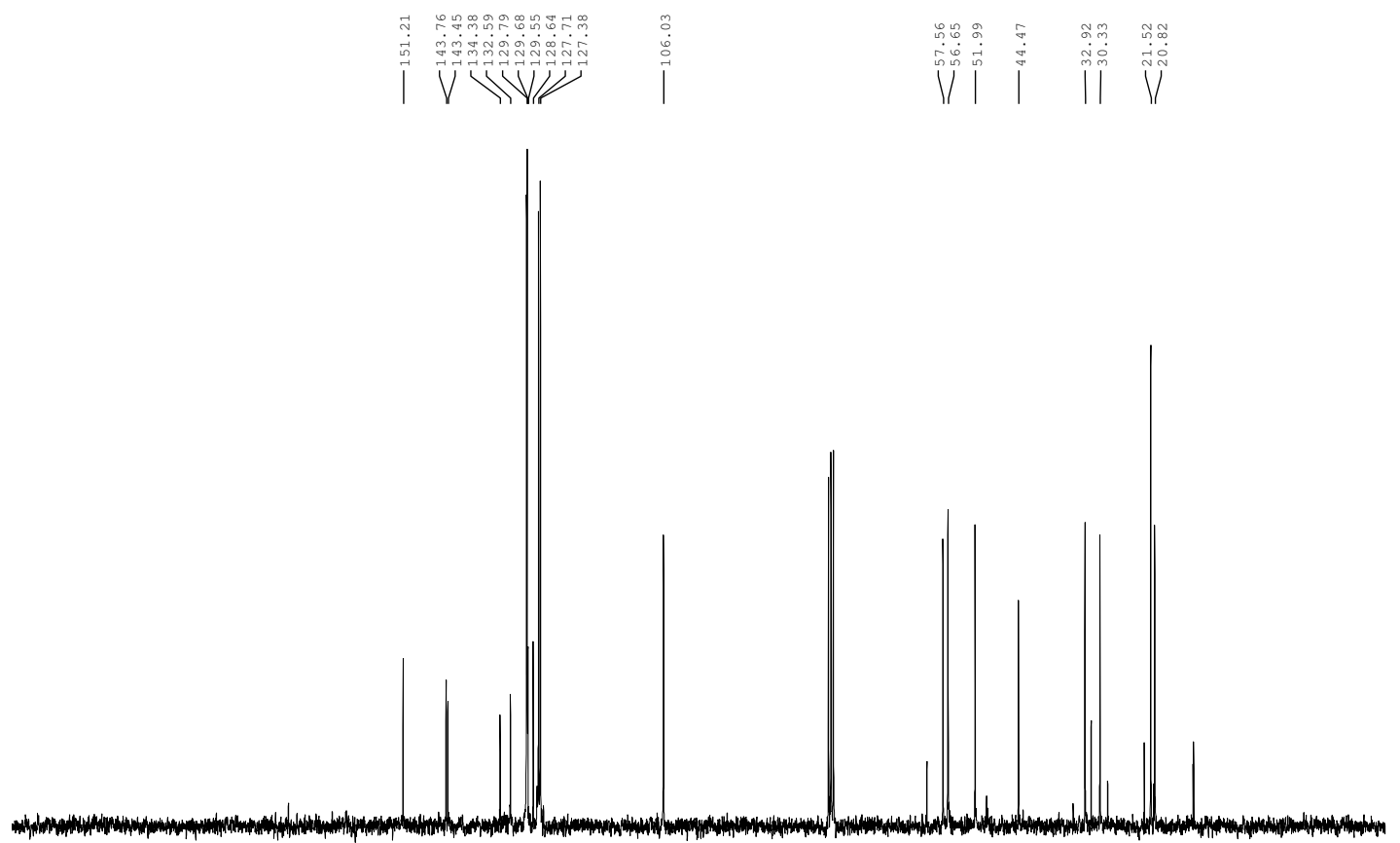

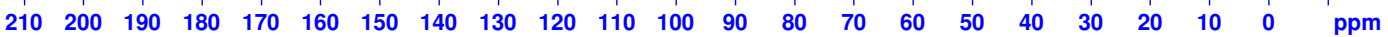


2b/2b', ${ }^{1} \mathbf{H} \mathbf{N M R}\left(300 \mathrm{MHz}, \mathrm{CDCl}_{3}\right)$

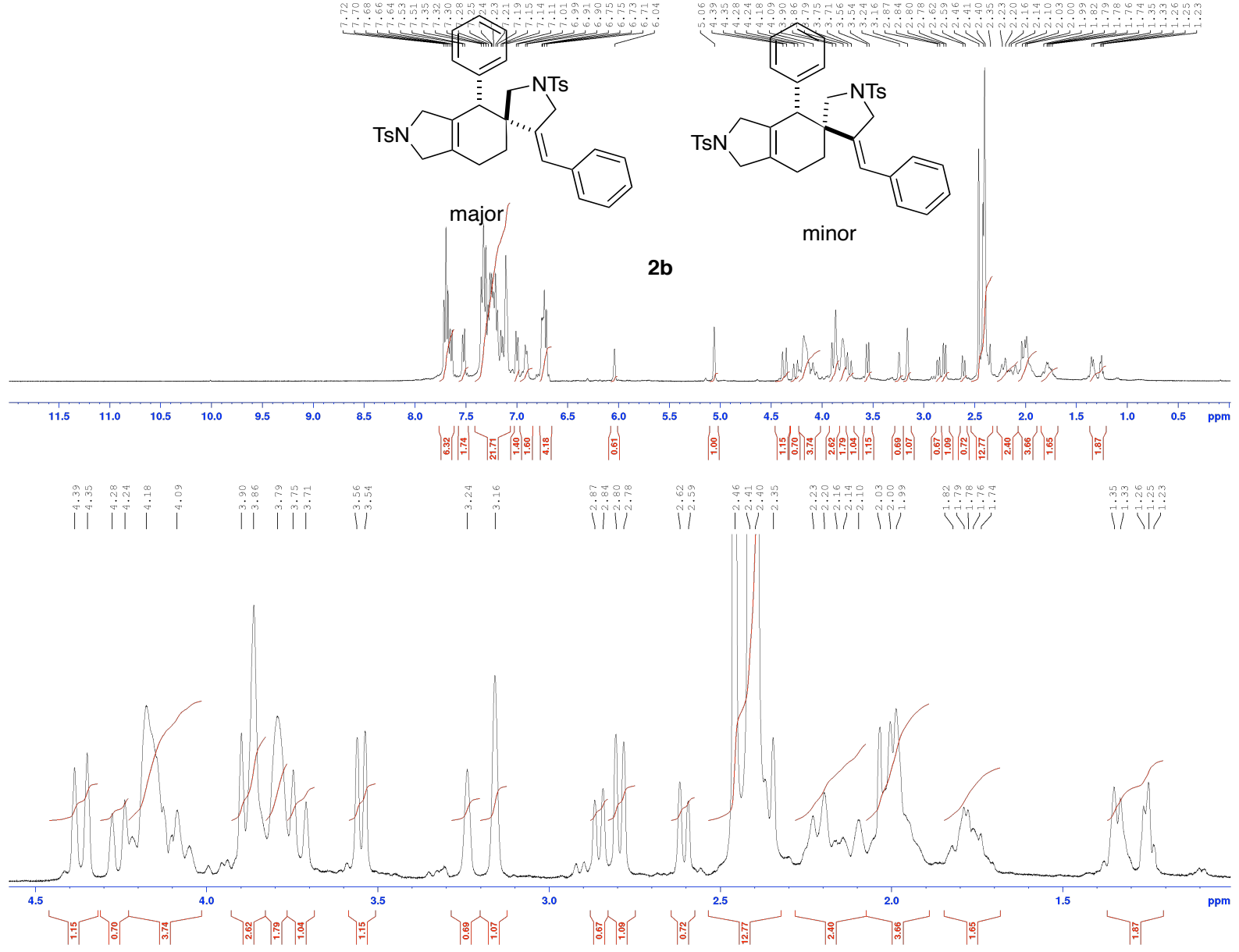

2b/2b, ${ }^{13} \mathbf{C}\{\mathbf{H}\}$ NMR $\left(75 \mathrm{MHz}, \mathrm{CDCl}_{3}\right)$

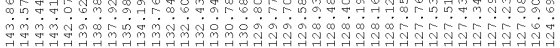

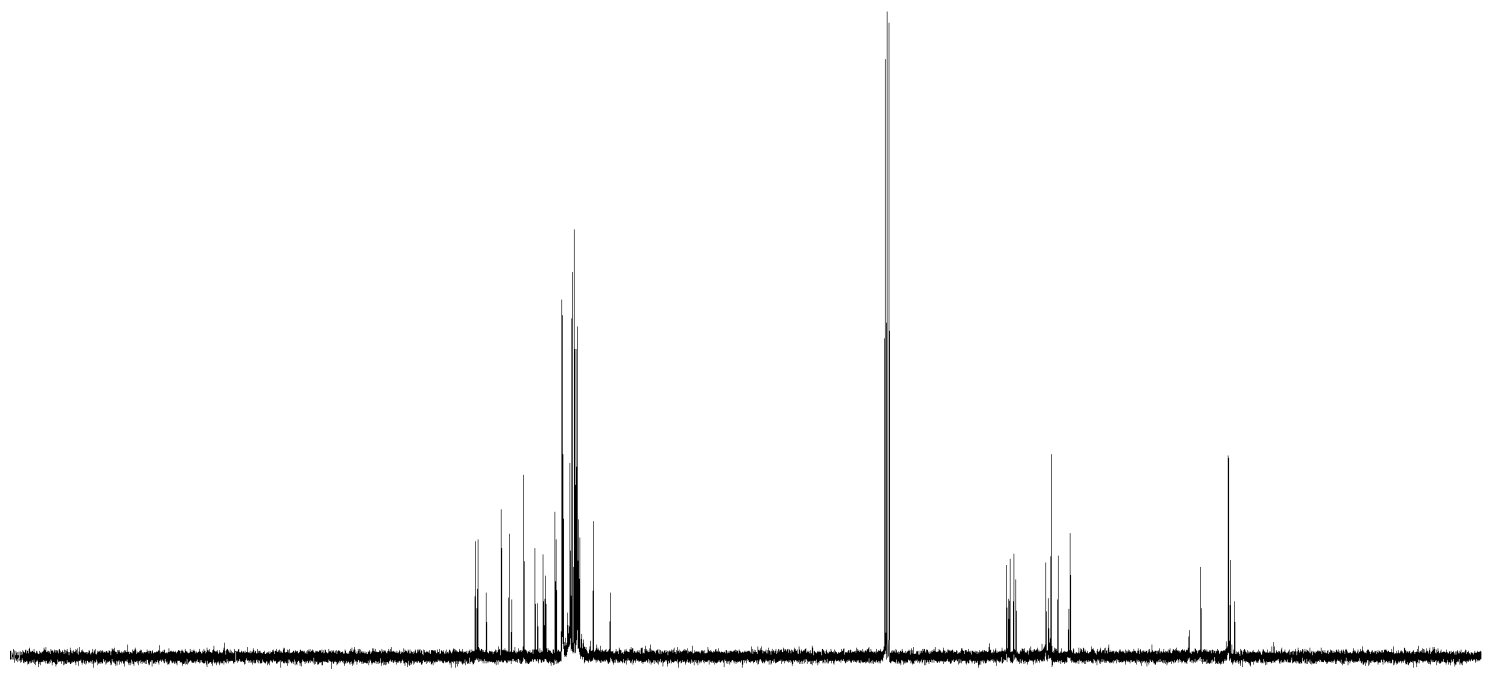

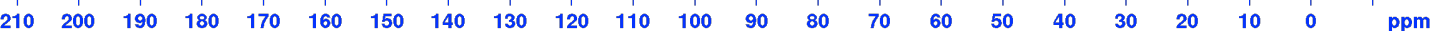


2c, ${ }^{1} \mathbf{H}$ NMR $\left(400 \mathrm{MHz}, \mathrm{CDCl}_{3}\right)$

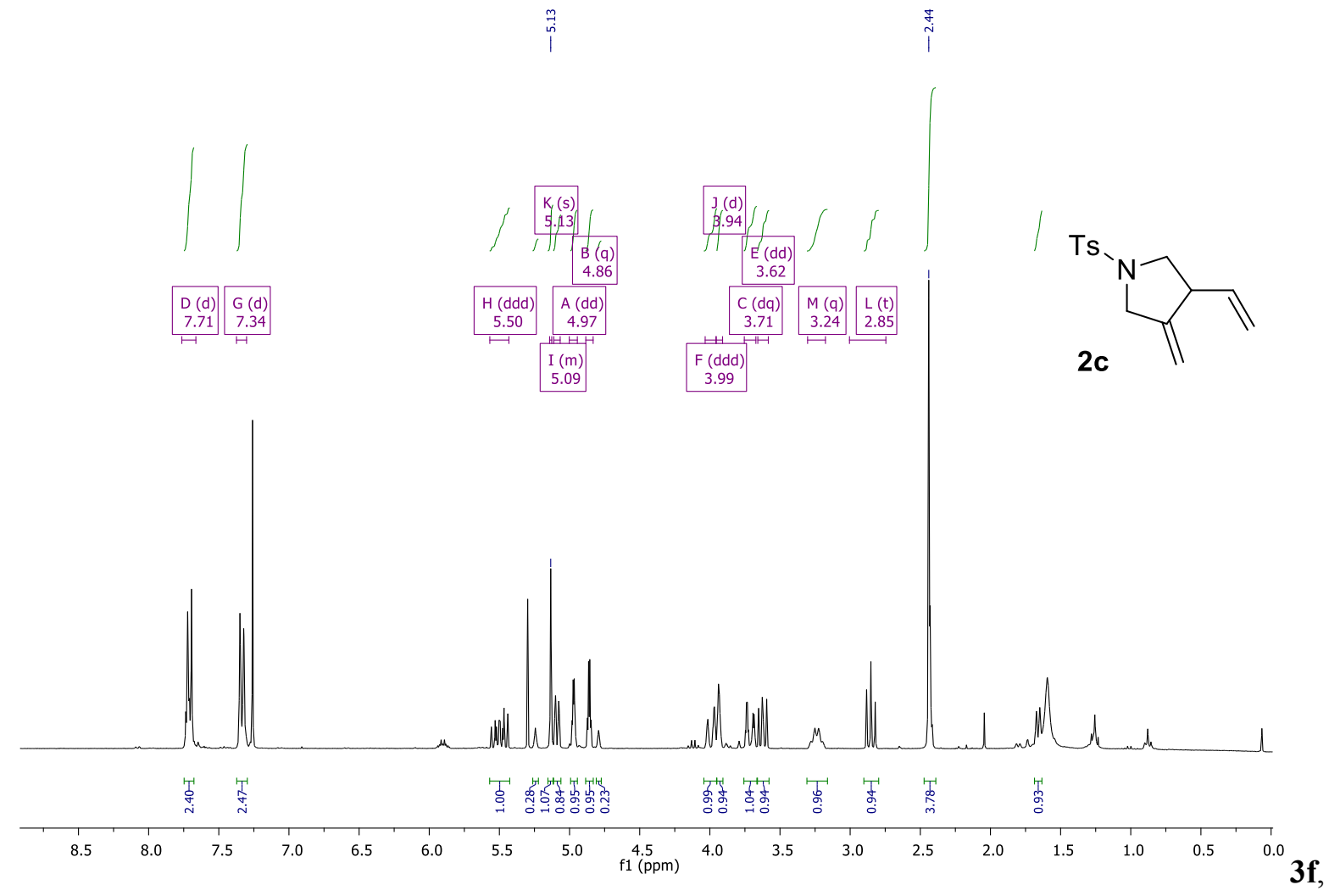

${ }^{13} \mathbf{C}\{\mathbf{H}\}$ NMR $\left(101 \mathrm{MHz}, \mathrm{CDCl}_{3}\right)$

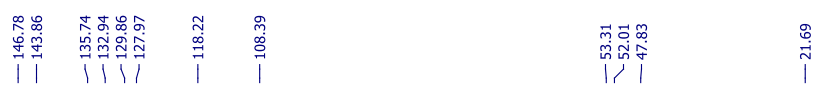

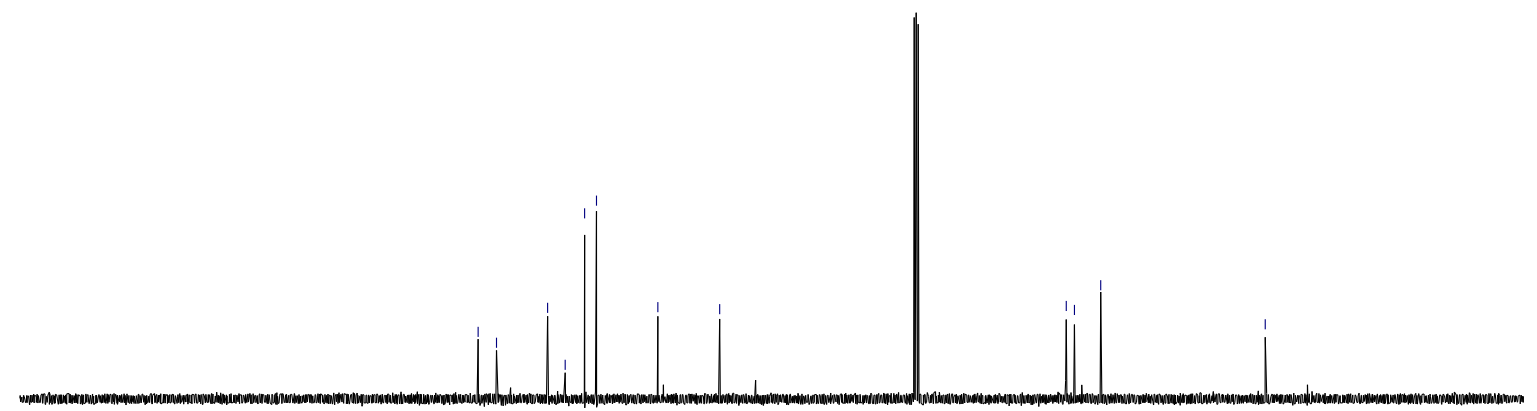

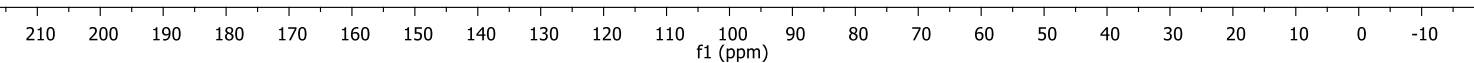


2d/2d', ${ }^{1}$ H NMR (300 MHz, Acetone- $\left.d_{6}\right)$

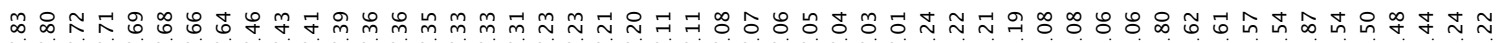

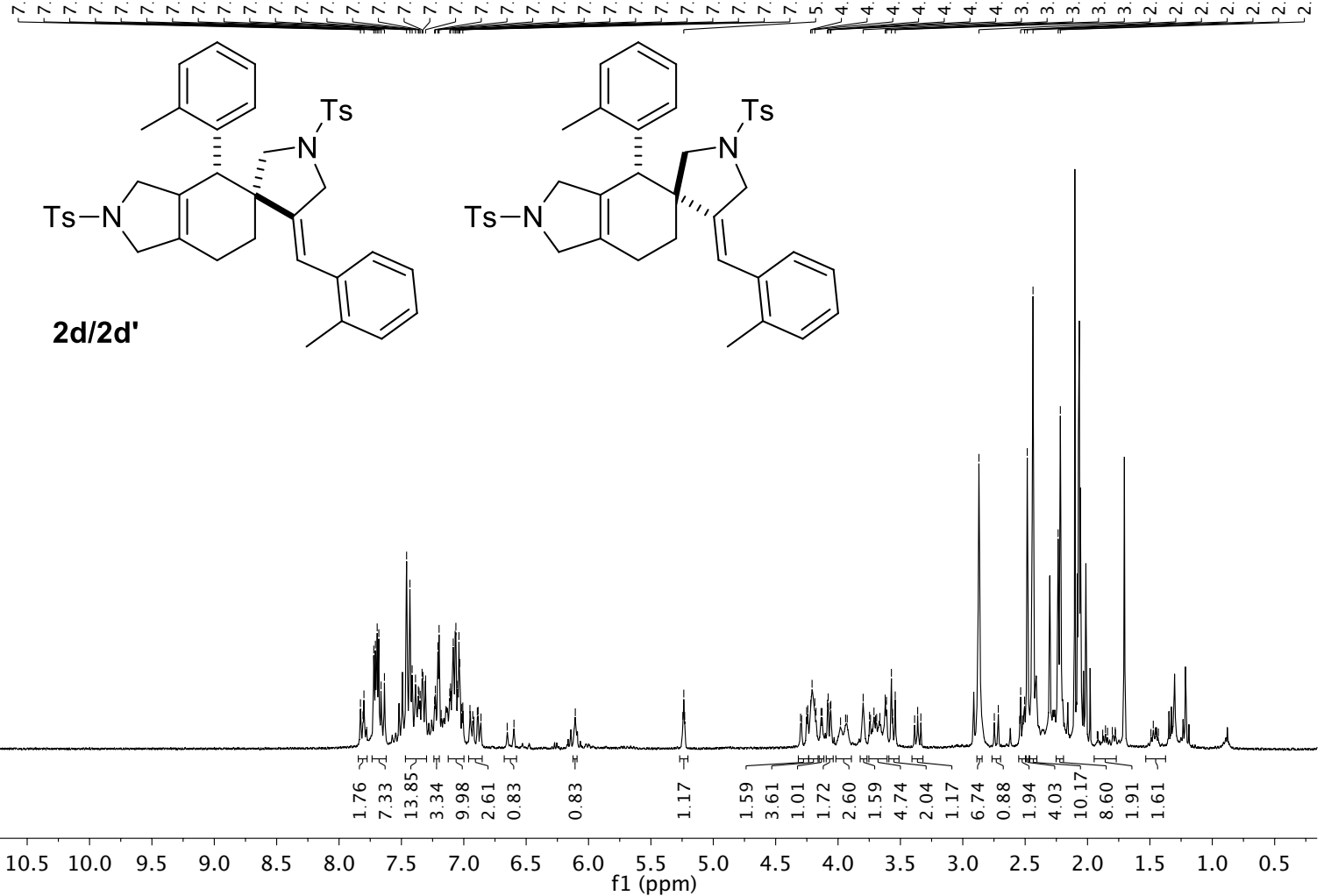

2d/2d', ${ }^{13} \mathbf{C}\{\mathbf{H}\}$ NMR $\left(75 \mathrm{MHz}\right.$, Acetone- $\left.d_{6}\right)$

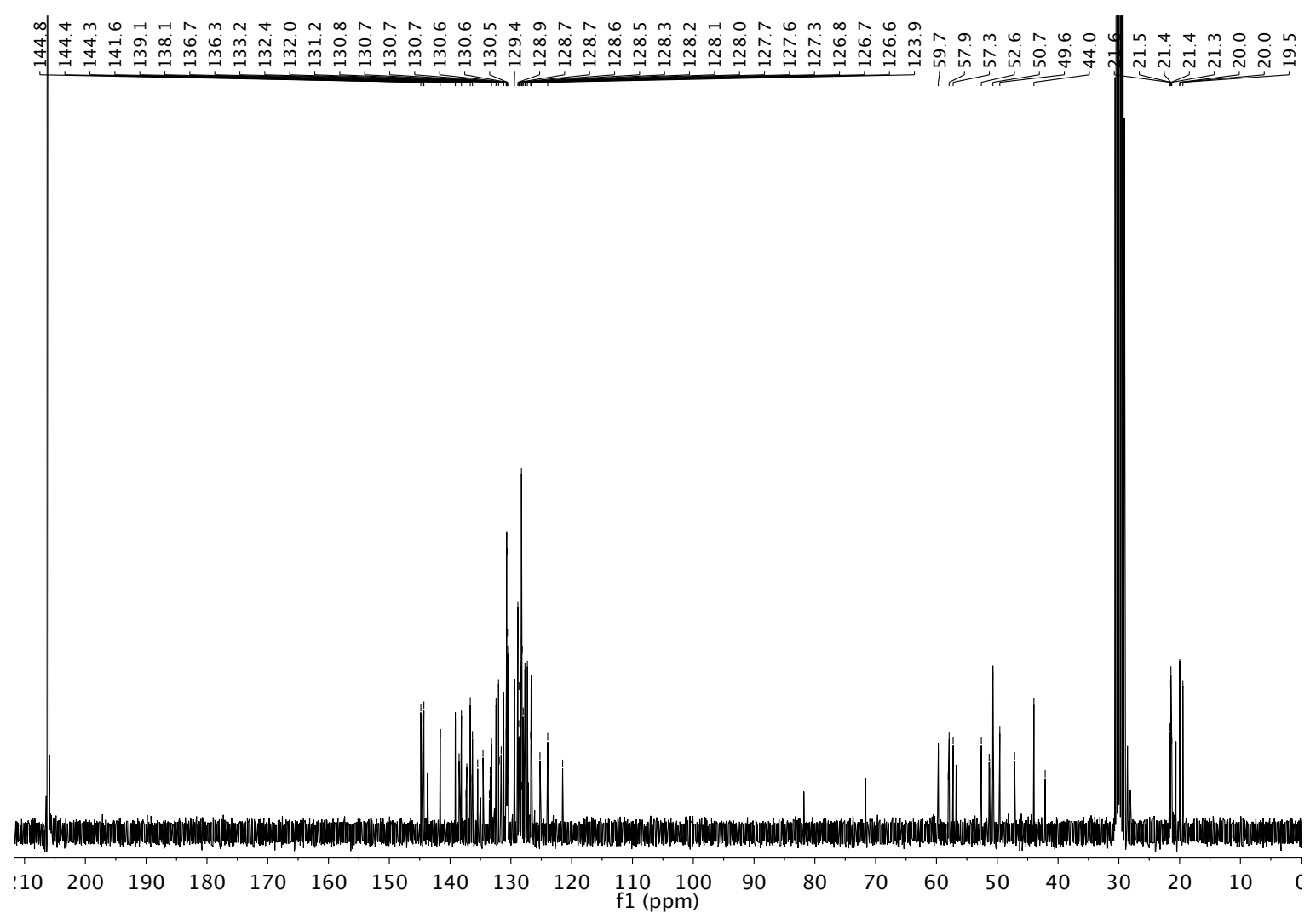


2e/2e', ${ }^{1} \mathbf{H}$ NMR (400 MHz, $\left.\mathrm{CDCl}_{3}\right)$

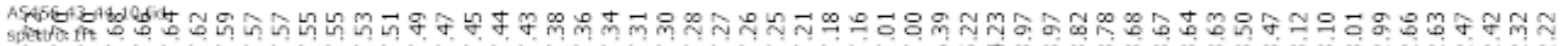

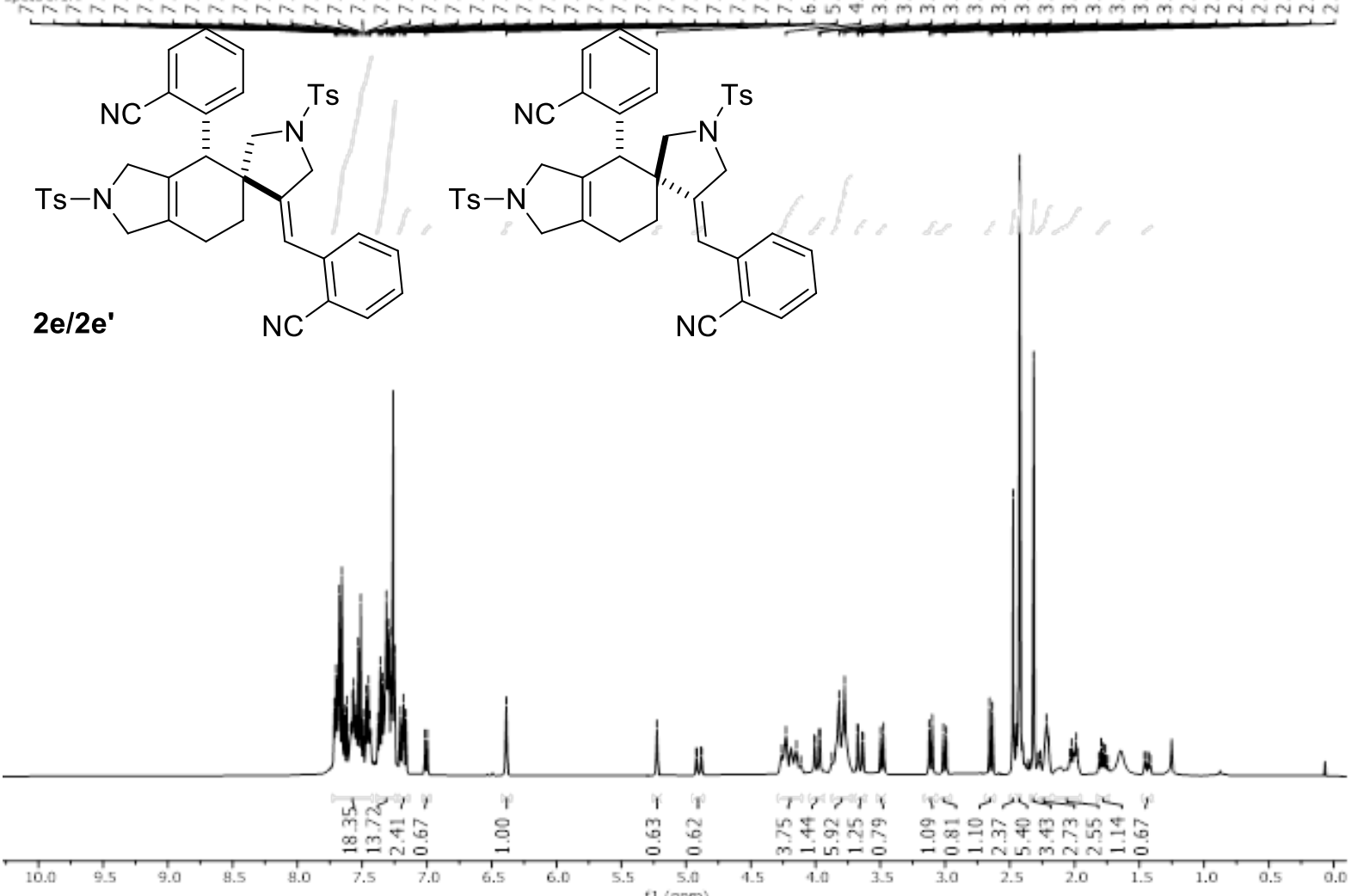

2e/2e', ${ }^{13} \mathbf{C}\{\mathbf{H}\}$ NMR $\left(101 \mathrm{MHz}, \mathrm{CDCl}_{3}\right)$

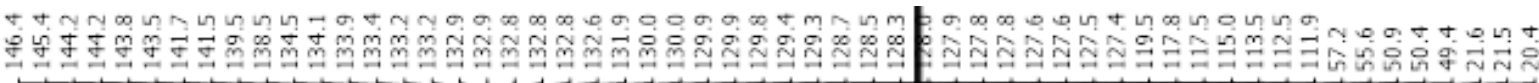

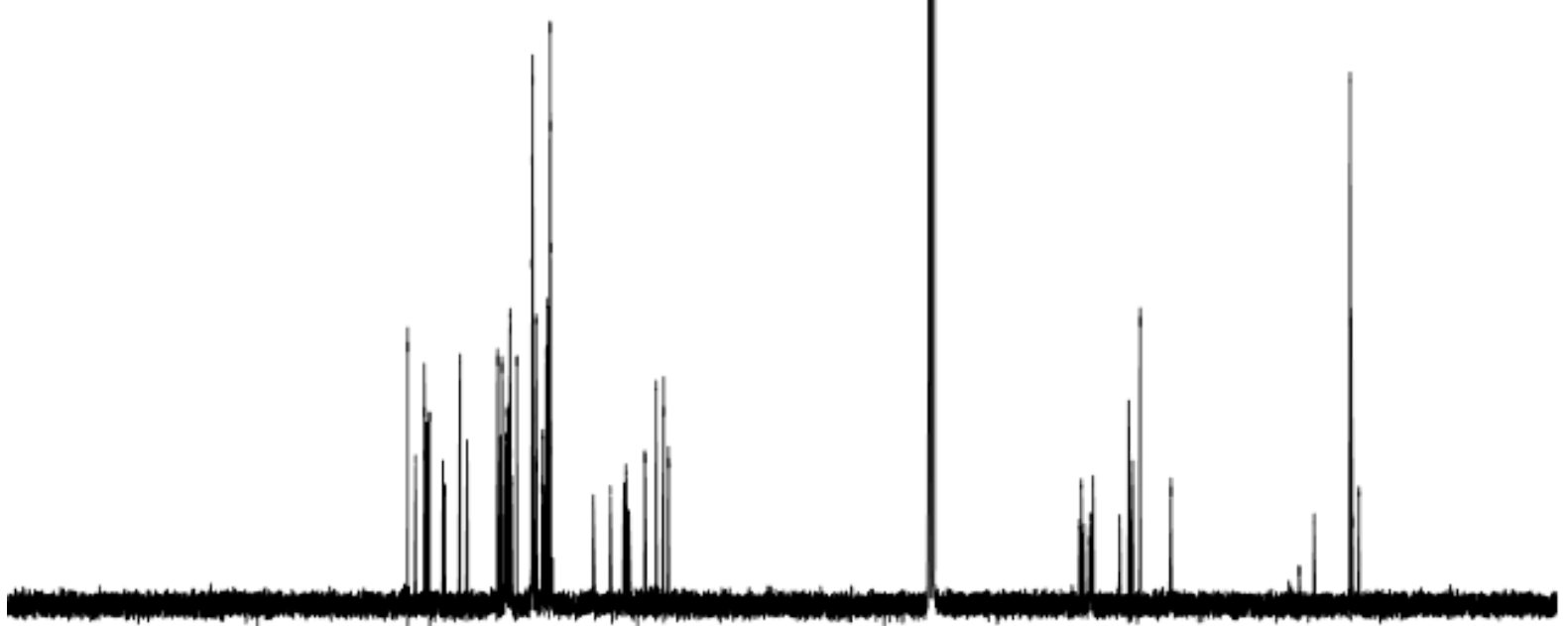


2f, ${ }^{1} \mathbf{H}$ NMR (400 MHz, $\left.\mathrm{CDCl}_{3}\right)$

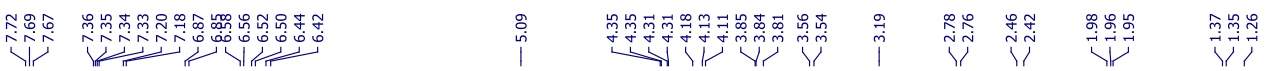

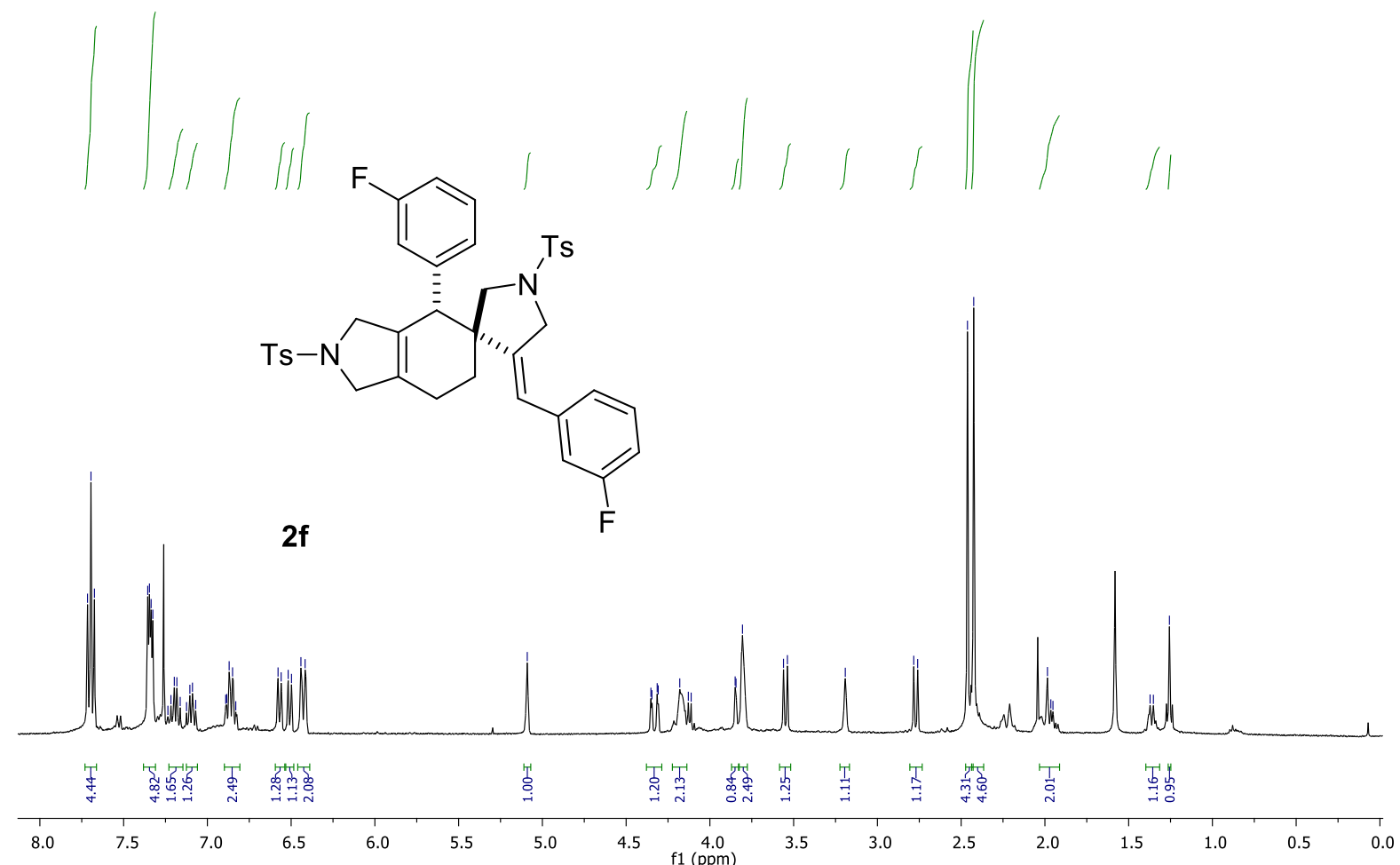

2f, ${ }^{13} \mathbf{C}\{\mathbf{H}\}$ NMR $\left(101 \mathrm{MHz}, \mathrm{CDCl}_{3}\right)$

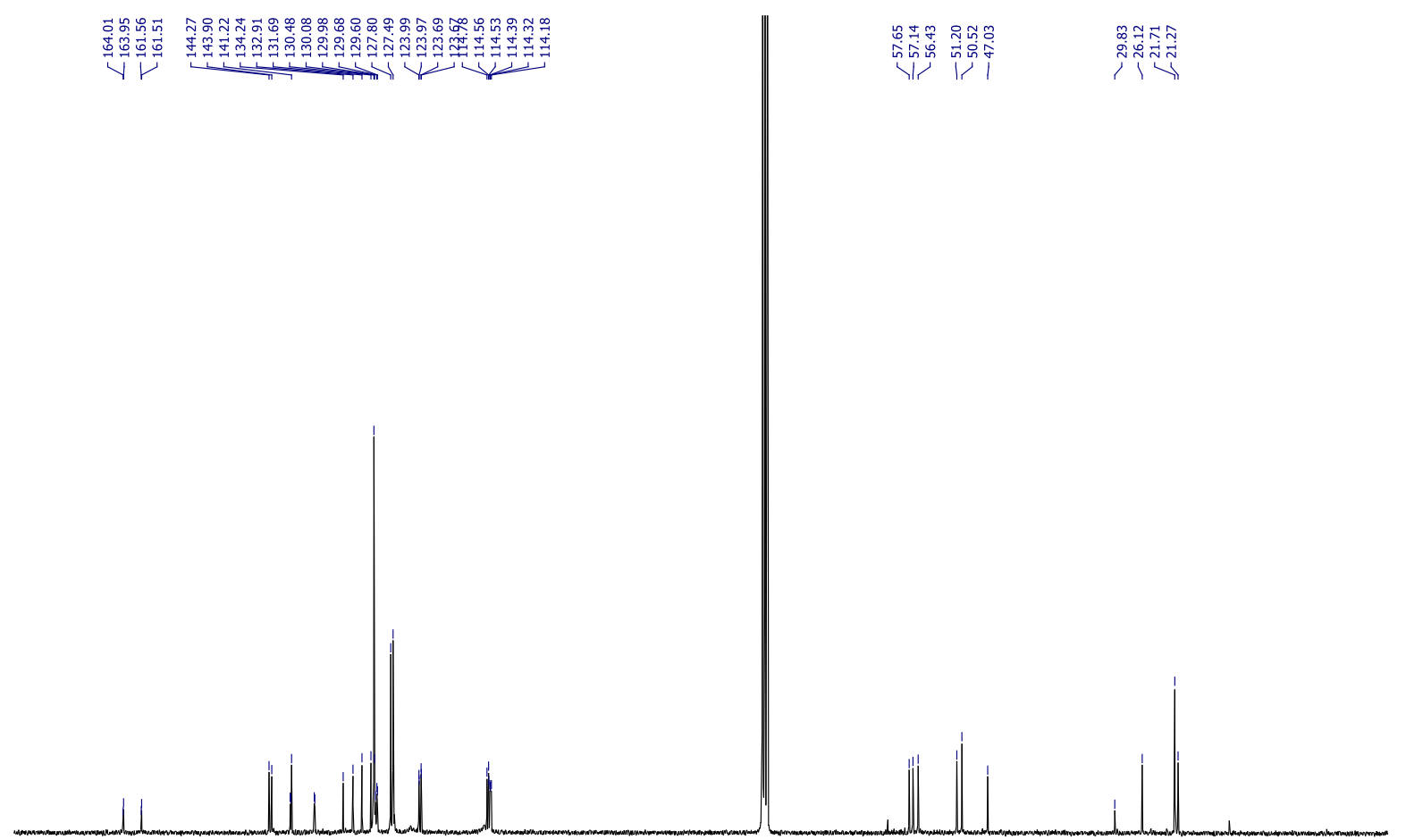

$\begin{array}{lllllllll}170 & 160 & 150 & 140 & 130 & 120 & 110 & 100 & 90 \\ & & & & & & & \end{array}$ 
2f, ${ }^{19} \mathbf{F}\{\mathbf{H}\}$ NMR $\left(376 \mathrm{MHz}, \mathrm{CDCl}_{3}\right)$<smiles>[Y5]N1CC2=C(C1)[C@H](c1cccc(F)c1)[C@@]1(CC2)CN([As])CC1=Cc1cccc(F)c1</smiles>

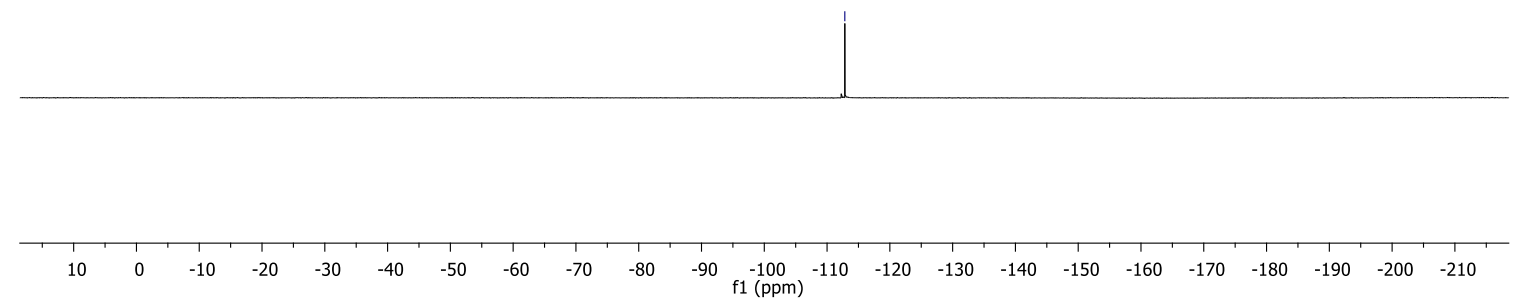


2f/2f', ${ }^{1} \mathbf{H}$ NMR (400 MHz, $\left.\mathrm{CDCl}_{3}\right)$

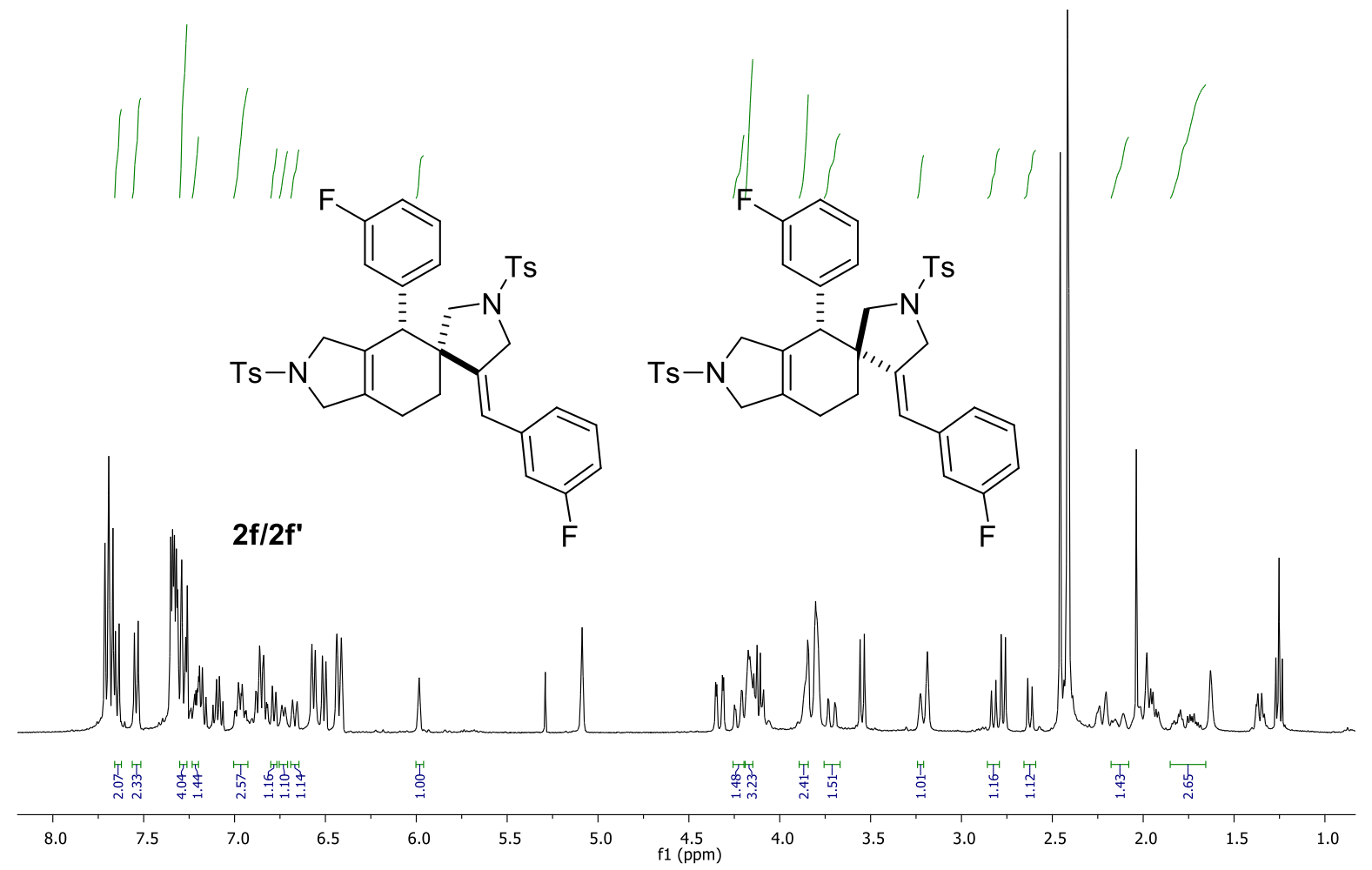


2f/2f', ${ }^{13} \mathbf{C}\{\mathbf{H}\}$ NMR $\left(101 \mathrm{MHz}, \mathrm{CDCl}_{3}\right)$
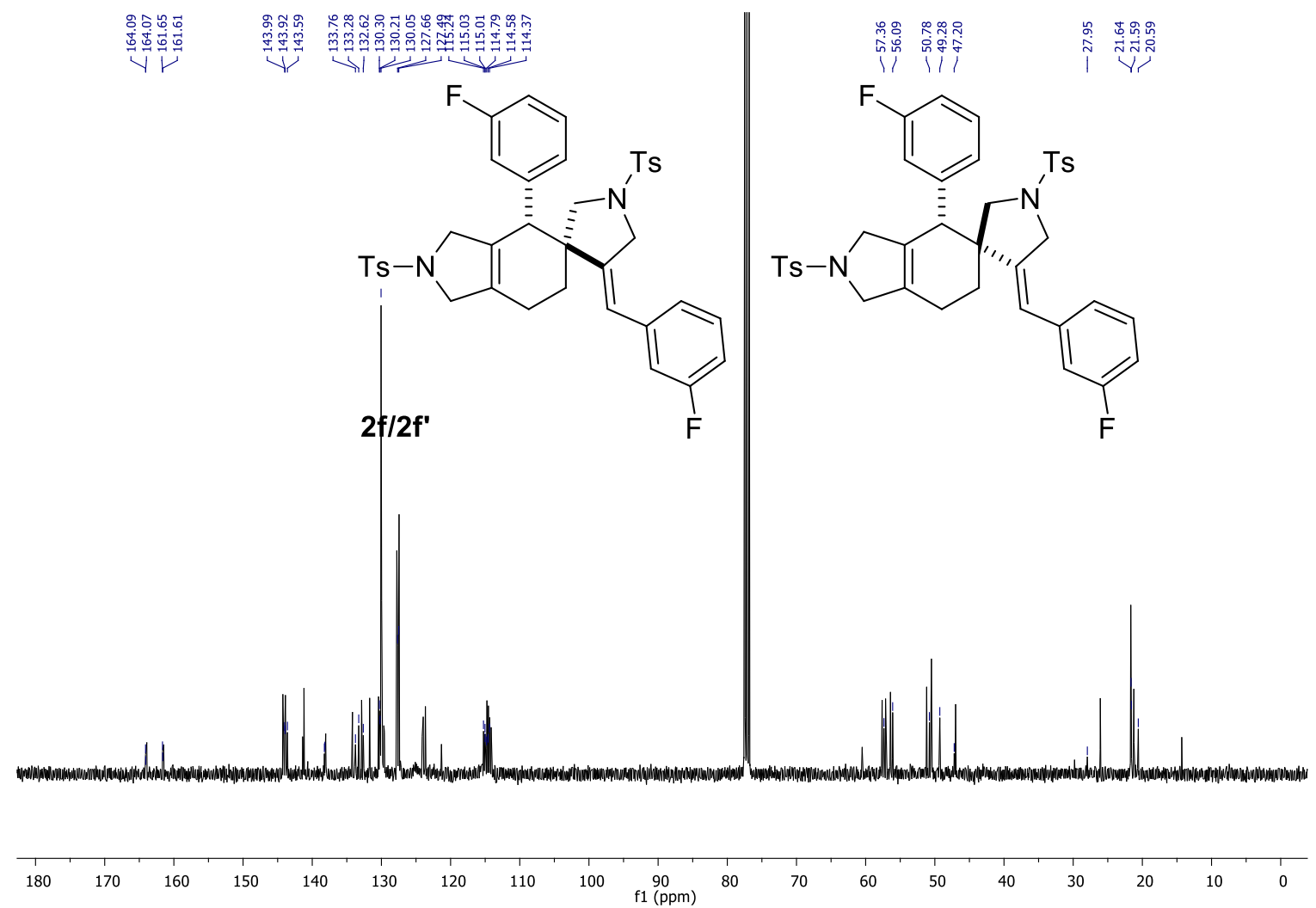

2f/2f, ${ }^{19} \mathbf{F}\{\mathbf{H}\} \mathbf{N M R}\left(376 \mathrm{MHz}, \mathrm{CDCl}_{3}\right)$

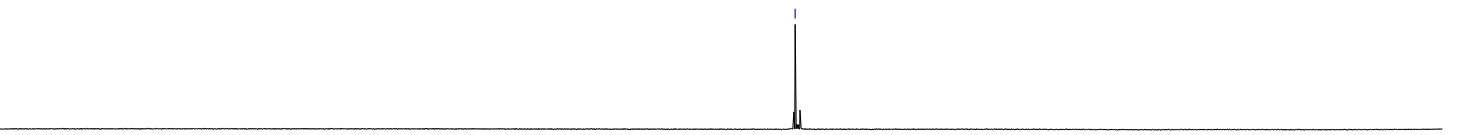

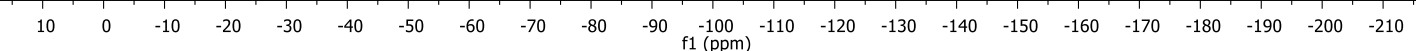


2g/2g, ${ }^{1} \mathbf{H}$ NMR $\left(400 \mathrm{MHz}, \mathrm{CDCl}_{3}\right)$

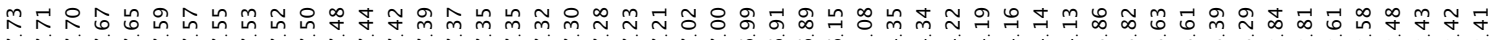

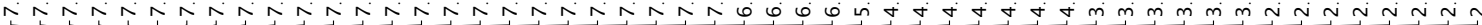<smiles>FC(F)(F)c1cccc(C=C2CN([As])C[C@@]23CCC2=C(CN([Tl])C2)[C@H]3c2cccc(C(F)(F)F)c2)c1</smiles><smiles>FC(F)(F)C1=C2CN([As])CC2=C1[C@]1(/C(=C/c2cccc(C(F)(F)F)c2)c2cccc(C(F)(F)F)c2)CN([As])C/C1=C\c1cccc(C(F)(F)F)c1</smiles>

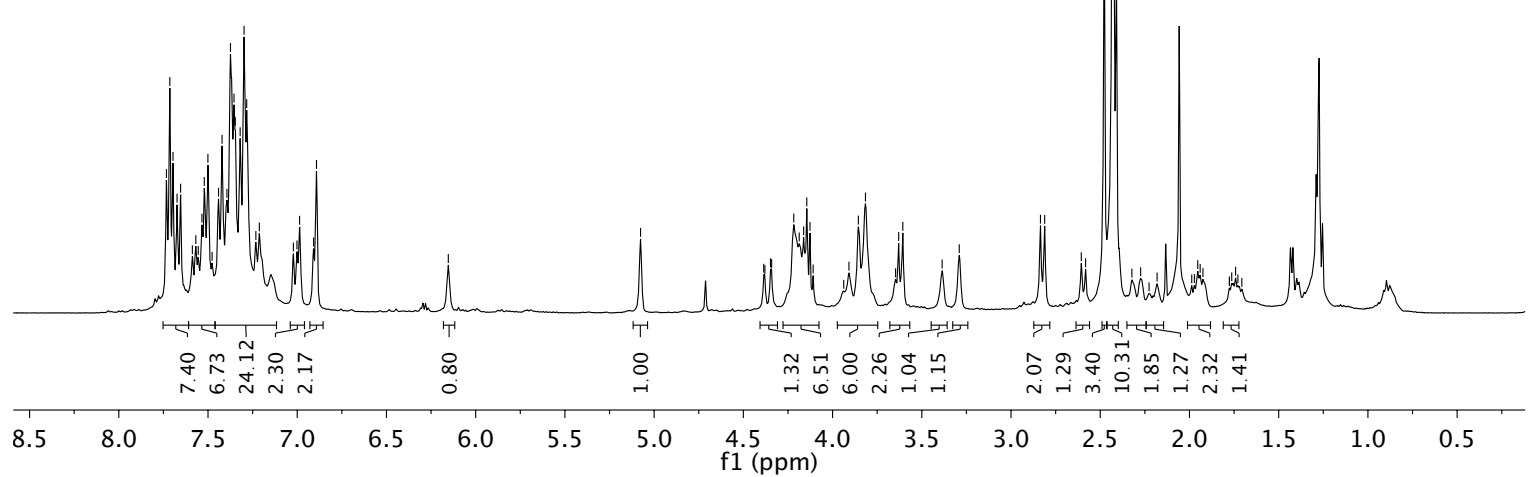

$\mathbf{2 g} / \mathbf{2 g},{ }^{13} \mathbf{C}\{\mathbf{H}\} \mathbf{N M R}\left(101 \mathrm{MHz}, \mathrm{CDCl}_{3}\right)$

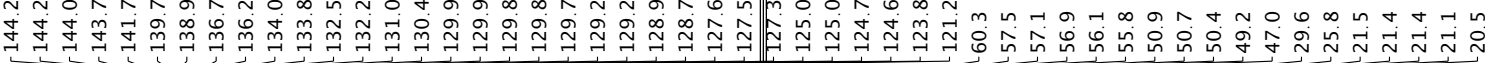

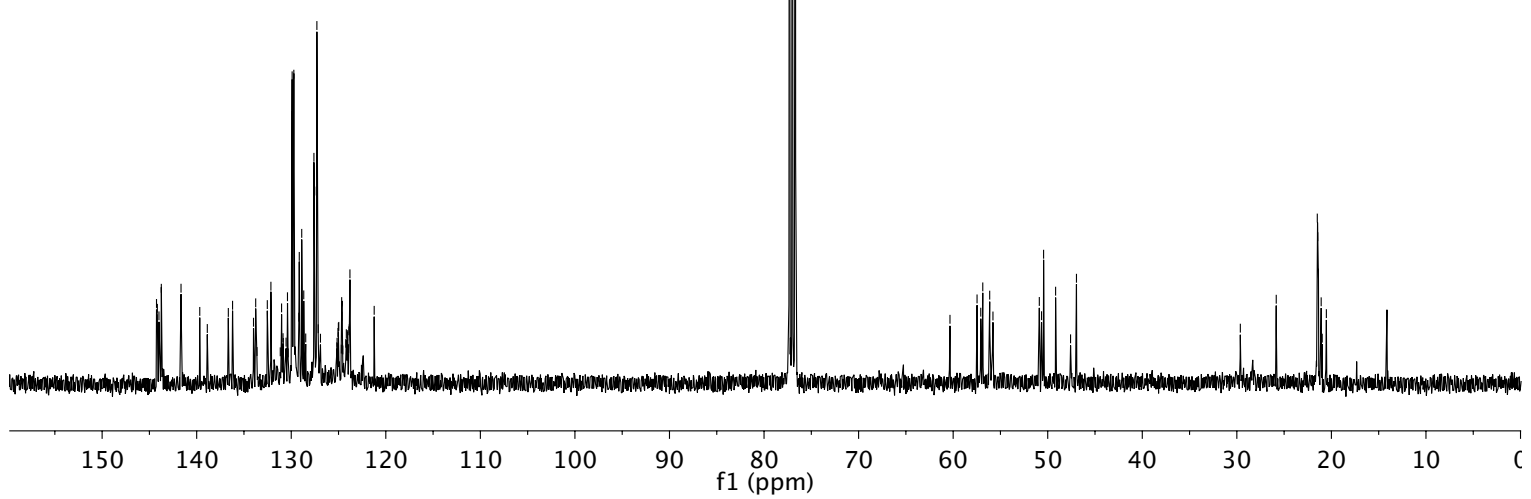


$\mathbf{2 g} / \mathbf{2 g},{ }^{19} \mathbf{F}\{\mathbf{H}\} \mathbf{N M R}\left(376 \mathrm{MHz}, \mathrm{CDCl}_{3}\right)$

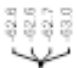

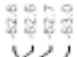

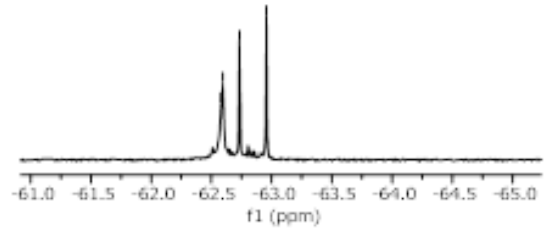<smiles>FC(F)(F)c1cccc(/C=C2\CN([As])C[C@]23CCC2=C(CN(S)C2)[C@H]3c2cccc(C(F)(F)F)c2)c1</smiles><smiles>FC(F)(F)c1cccc(C=C2CN([AlH2])C[C@]23CCC2=C(CN([As])C2)[C@H]3c2cccc(C(F)(F)F)c2)c1</smiles>

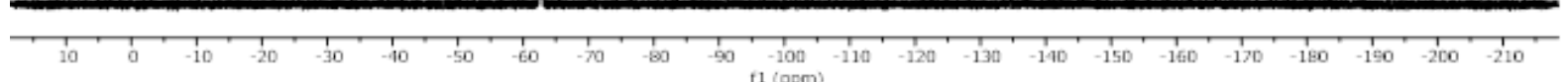


2h/2h', ${ }^{1} \mathbf{H}$ NMR $\left(400 \mathrm{MHz}, \mathrm{CDCl}_{3}\right)$

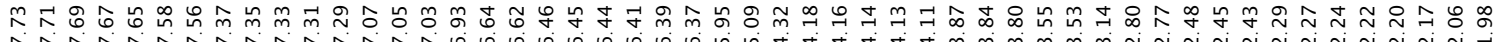

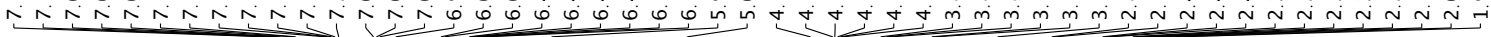

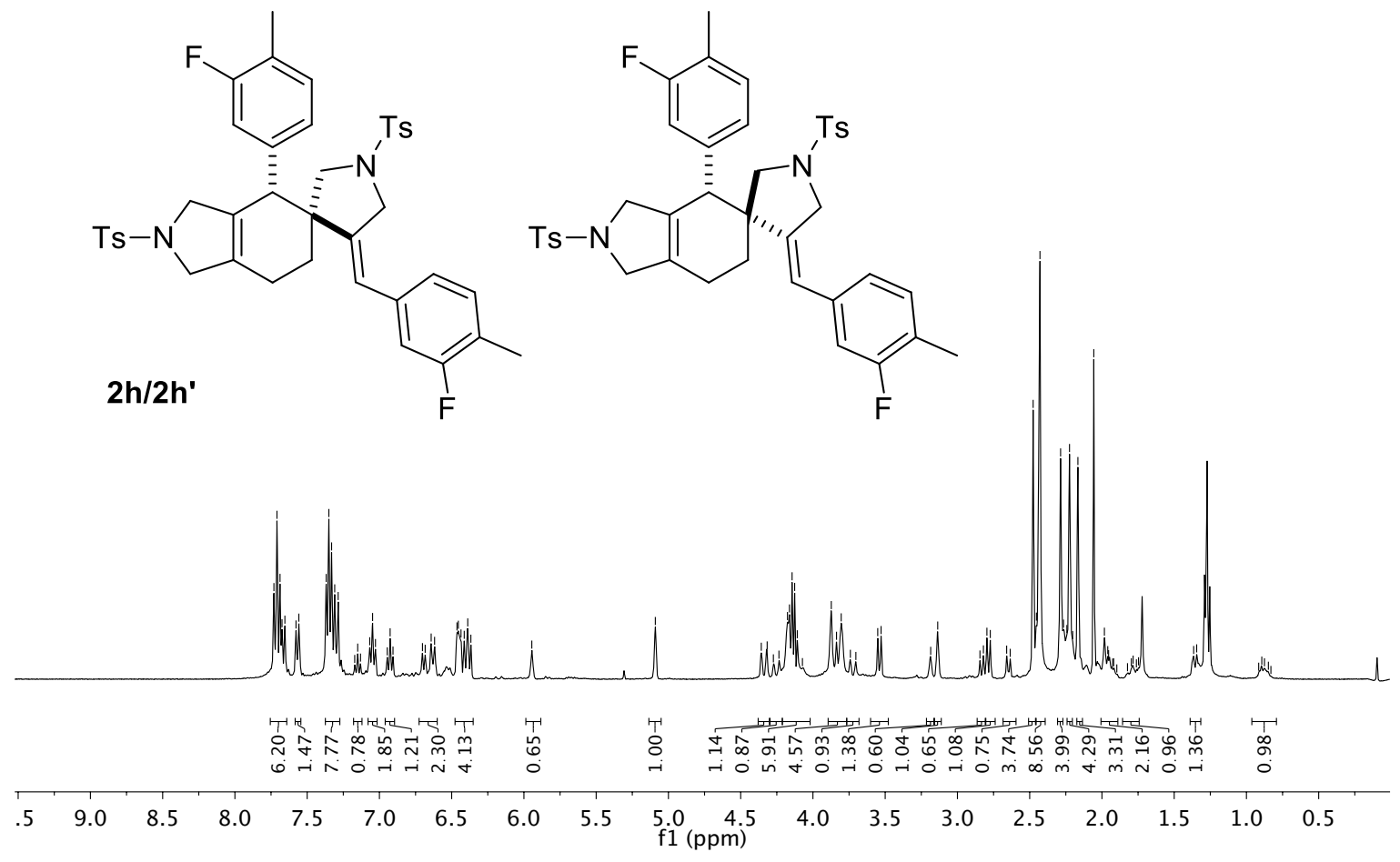


2h/2h', ${ }^{13} \mathbf{C}\{\mathbf{H}\} \mathbf{N M R}\left(101 \mathrm{MHz}, \mathrm{CDCl}_{3}\right)$

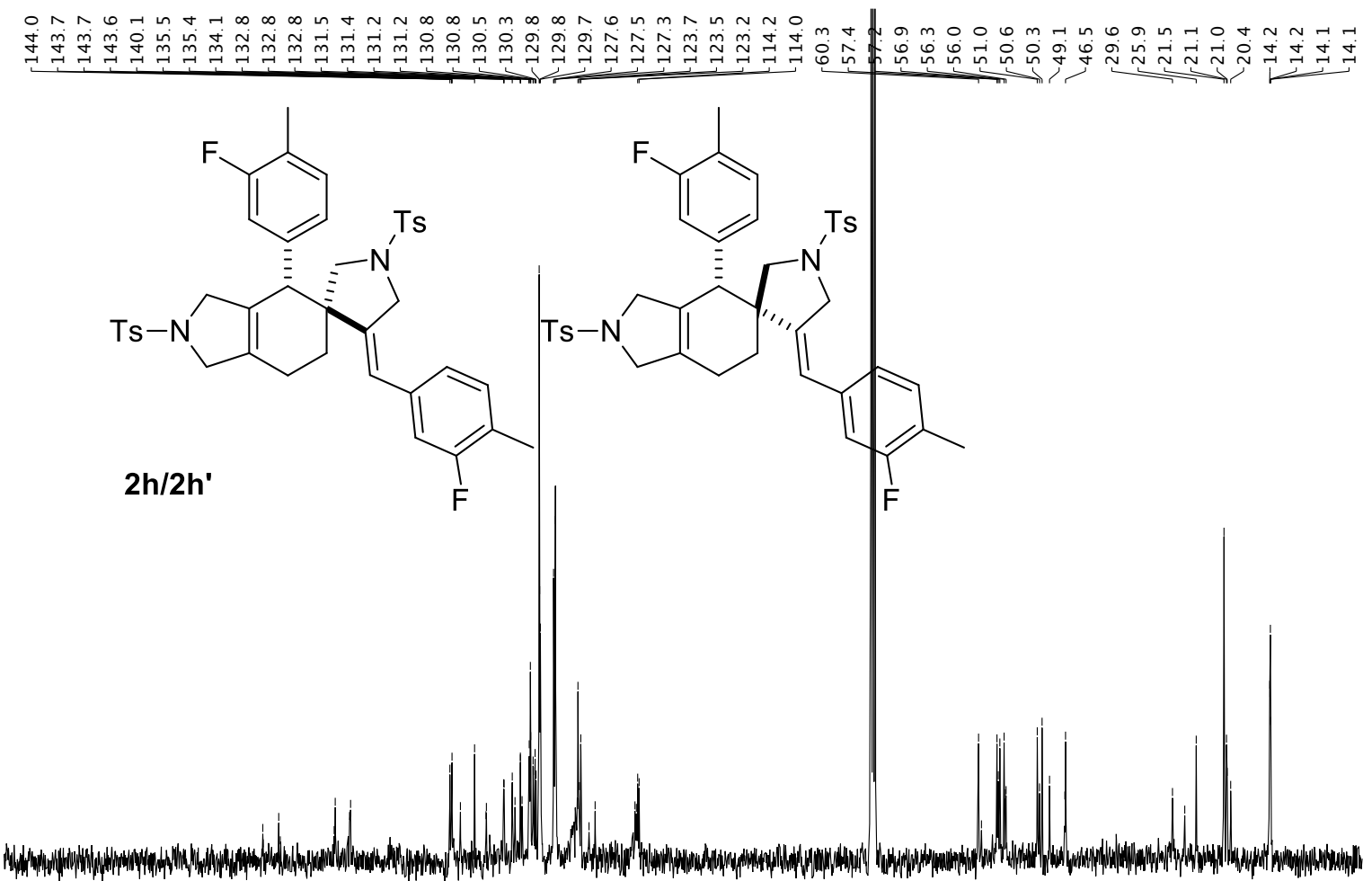

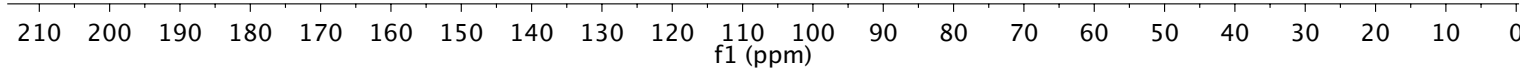

2h/2h', ${ }^{19} \mathbf{F}\{\mathbf{H}\} \mathbf{N M R}\left(376 \mathrm{MHz}, \mathrm{CDCl}_{3}\right)$

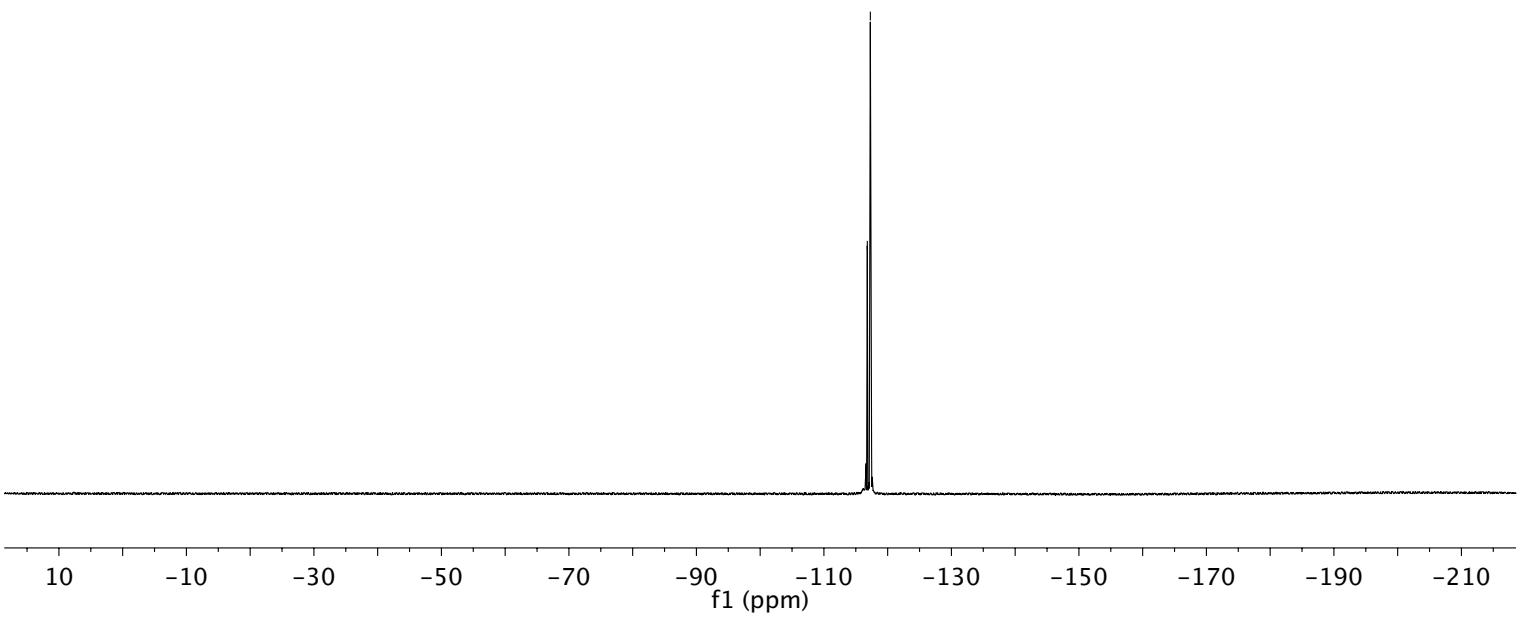


2i/2i', ${ }^{1} \mathbf{H}$ NMR (400 MHz, $\left.\mathrm{CDCl}_{3}\right)$

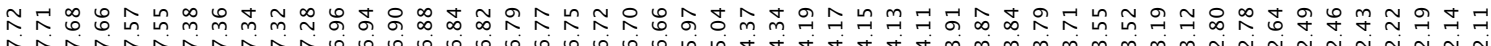

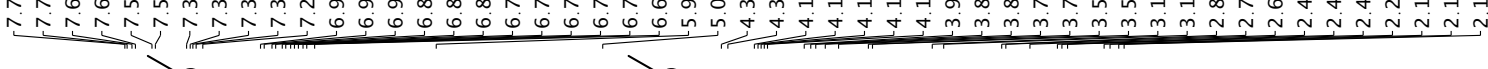

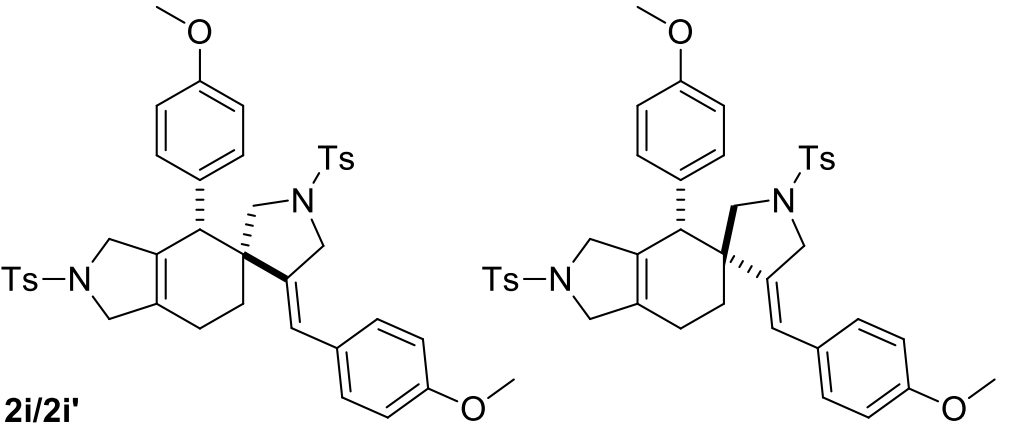

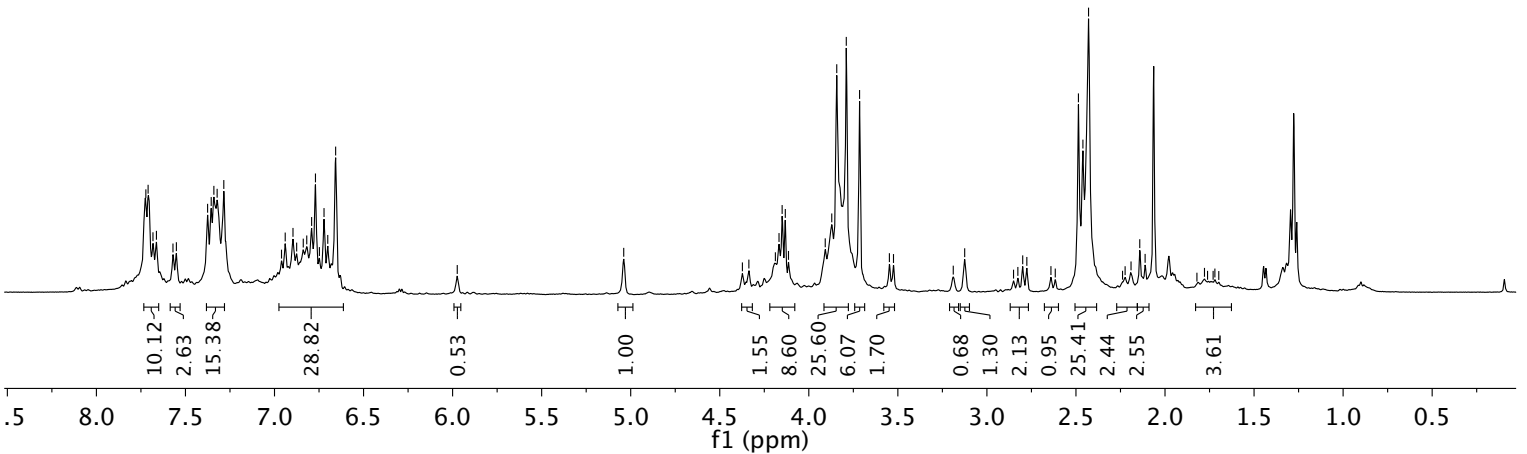

2i/2i', ${ }^{13} \mathbf{C}\{\mathbf{H}\} \mathbf{N M R}\left(101 \mathrm{MHz}, \mathrm{CDCl}_{3}\right)$

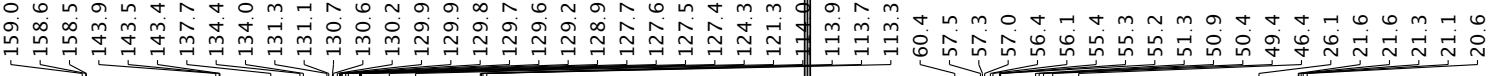

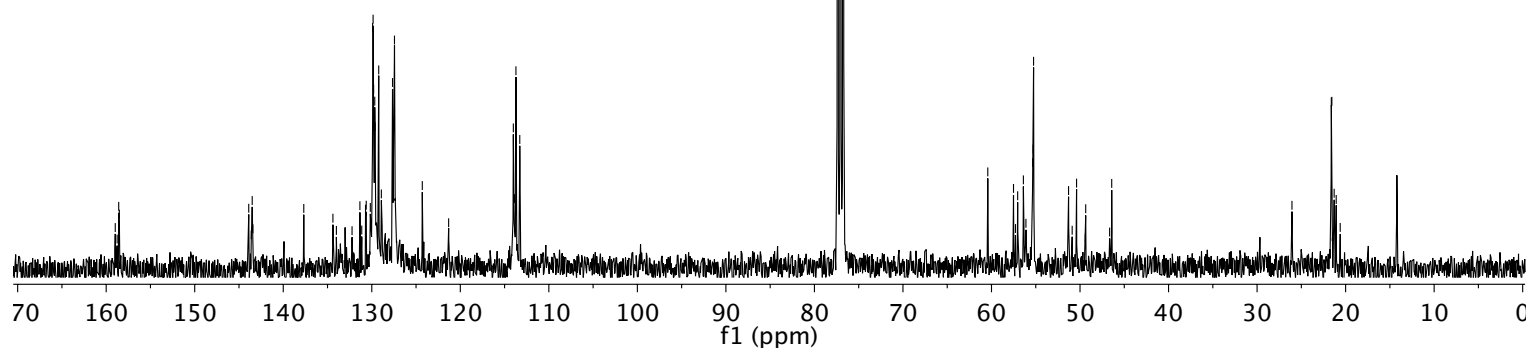


2j/2j', ${ }^{\mathbf{1}} \mathbf{H}$ NMR (400 MHz, $\left.\mathrm{CDCl}_{3}\right)$

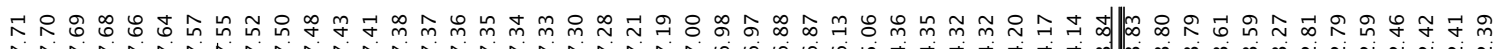

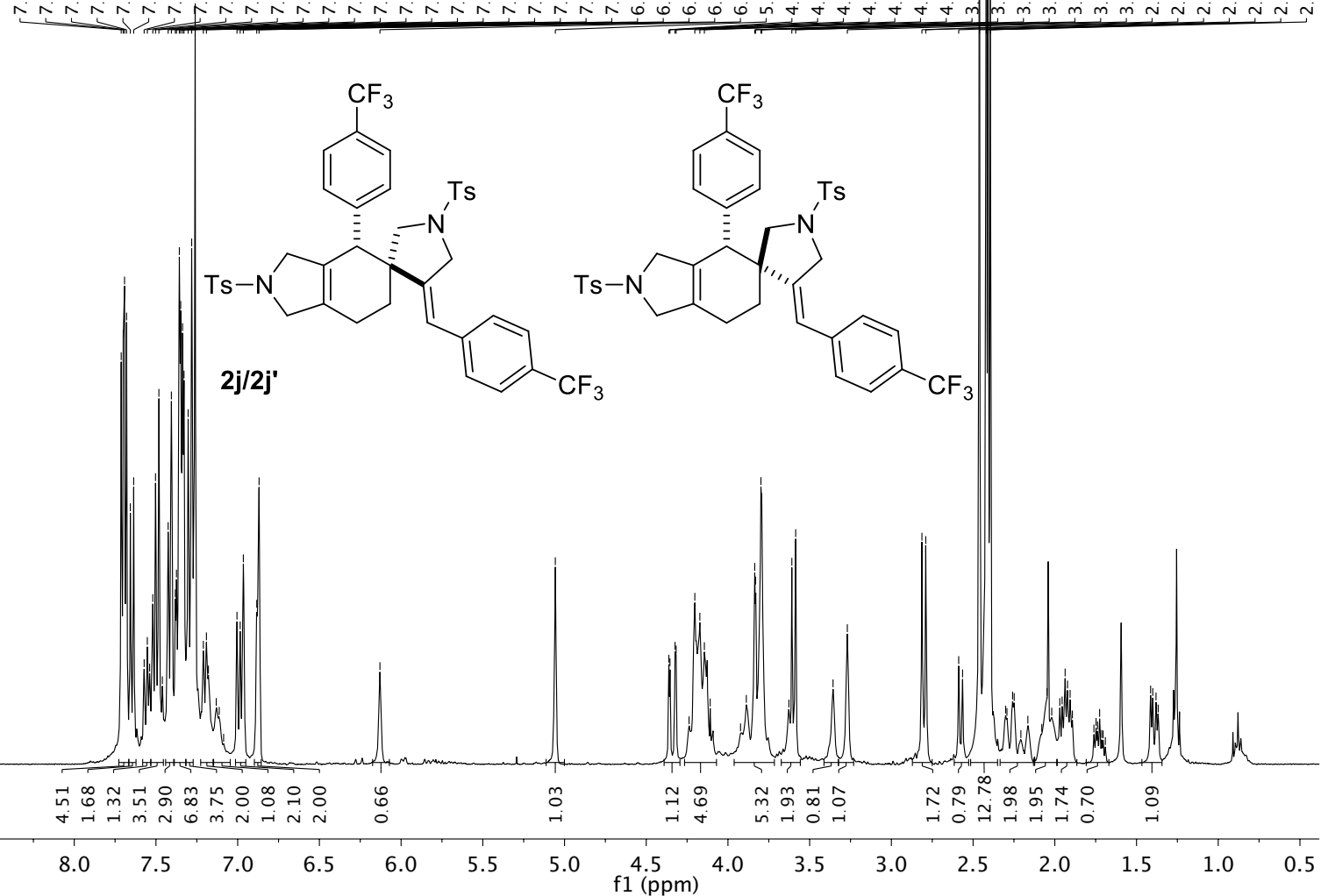


$\mathbf{2} \mathbf{j} / \mathbf{2} \mathbf{j},{ }^{13} \mathbf{C}\{\mathbf{H}\} \mathbf{N M R}\left(101 \mathrm{MHz}, \mathrm{CDCl}_{3}\right)$

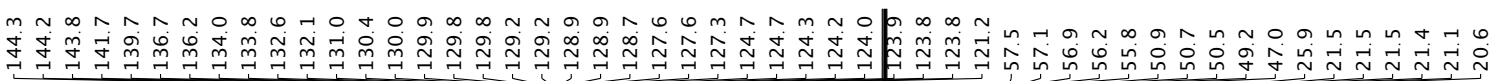

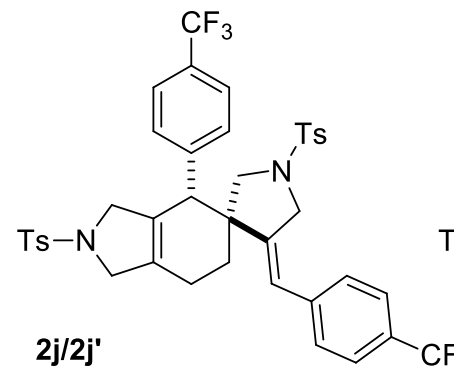

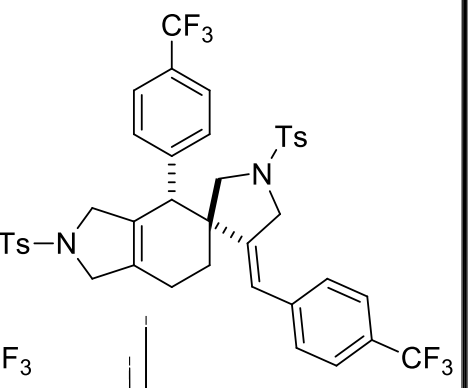

H.

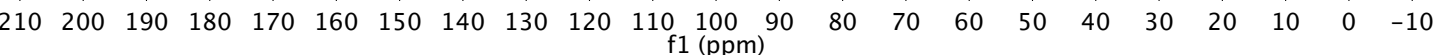

$\mathbf{2 j} / \mathbf{2} \mathbf{j},{ }^{19} \mathbf{F}\{\mathbf{H}\} \mathbf{N M R}\left(376 \mathrm{MHz}, \mathrm{CDCl}_{3}\right)$

$0 \infty 0$

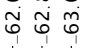

r

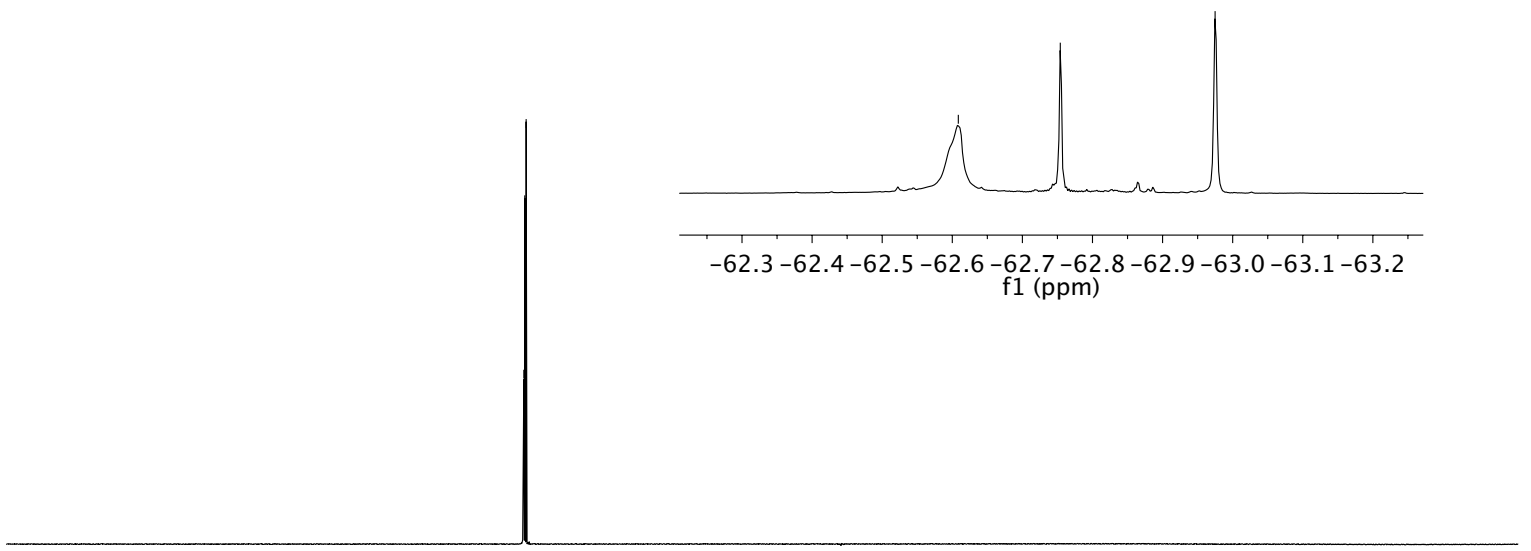

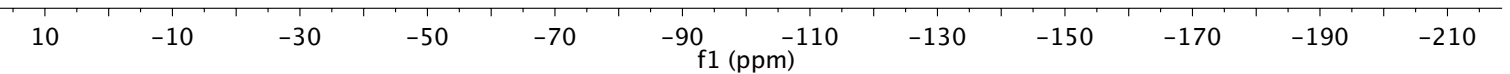


2k/2k', ${ }^{1} \mathbf{H}$ NMR $\left(400 \mathrm{MHz}, \mathrm{CDCl}_{3}\right)$

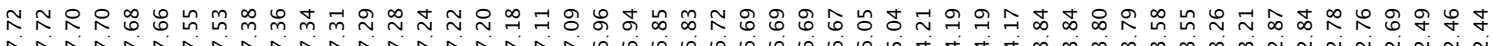

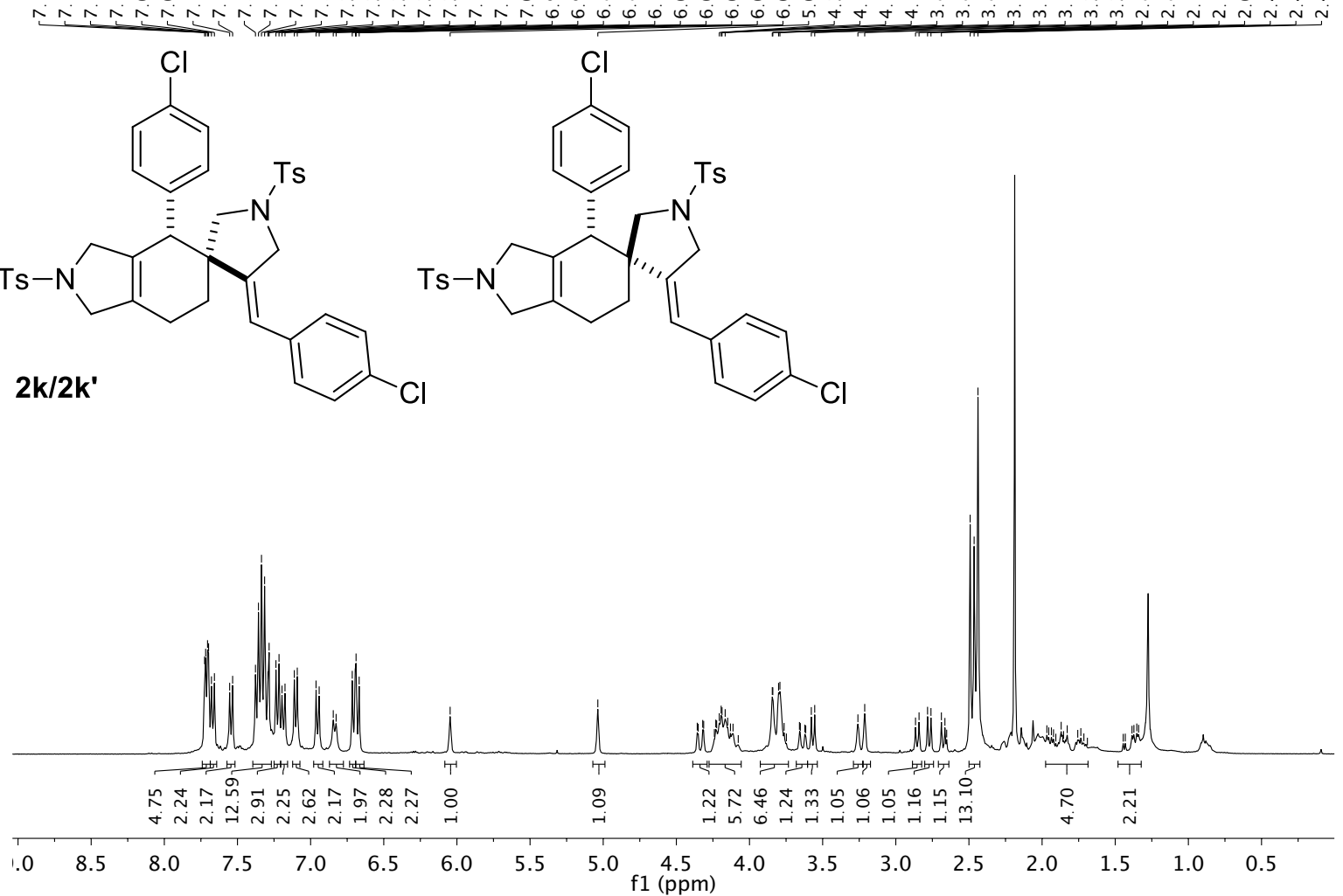

2k/2k, ${ }^{13} \mathbf{C}\{\mathbf{H}\} \mathbf{N M R}\left(101 \mathrm{MHz}, \mathrm{CDCl}_{3}\right)$

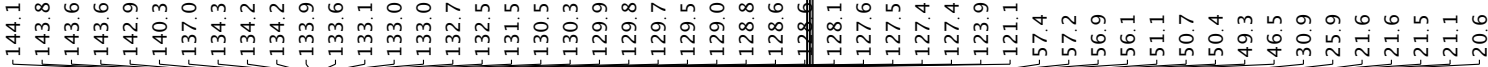

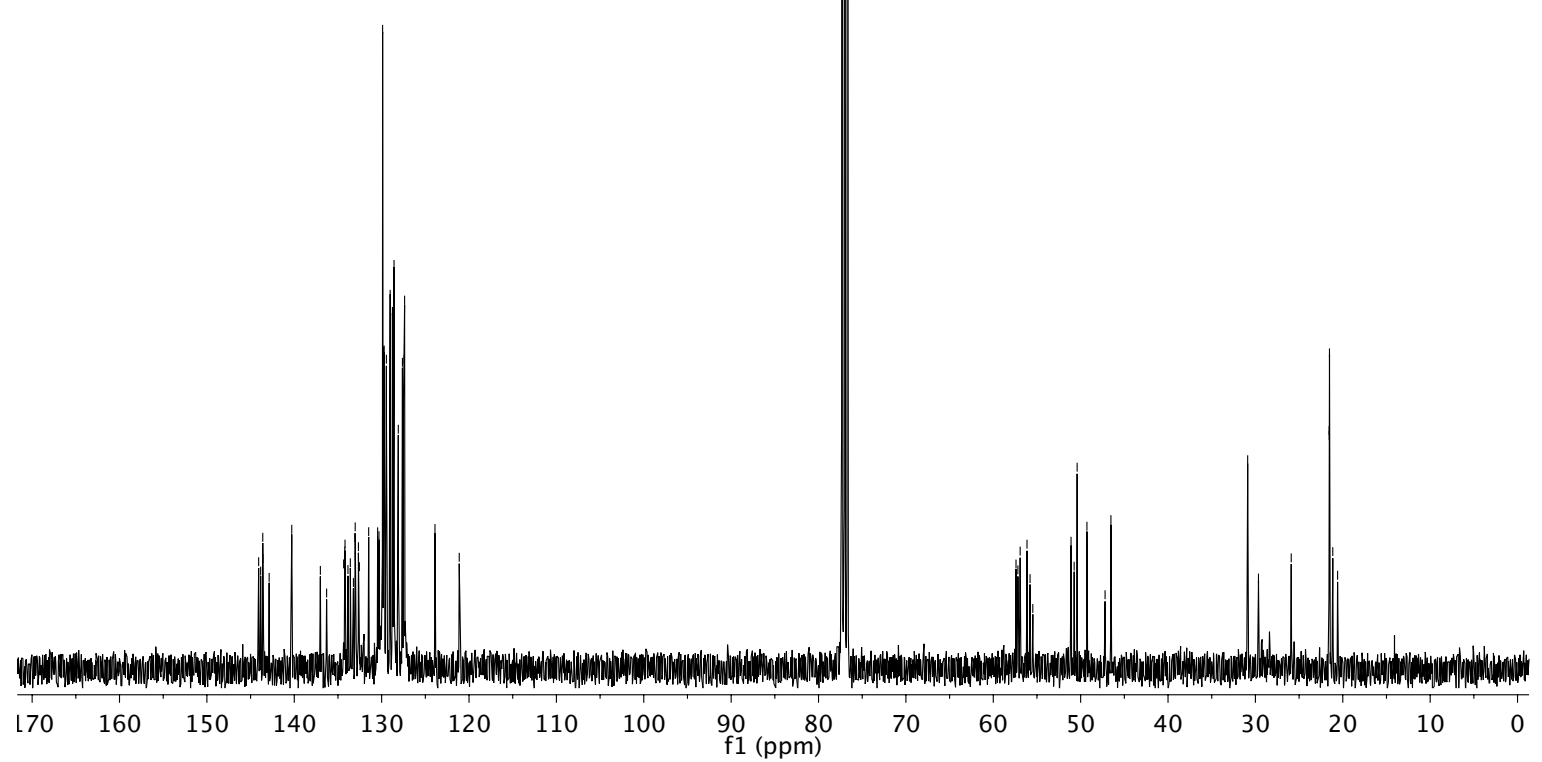


21, ${ }^{1} \mathbf{H}$ NMR (400 MHz, $\left.\mathrm{CDCl}_{3}\right)$

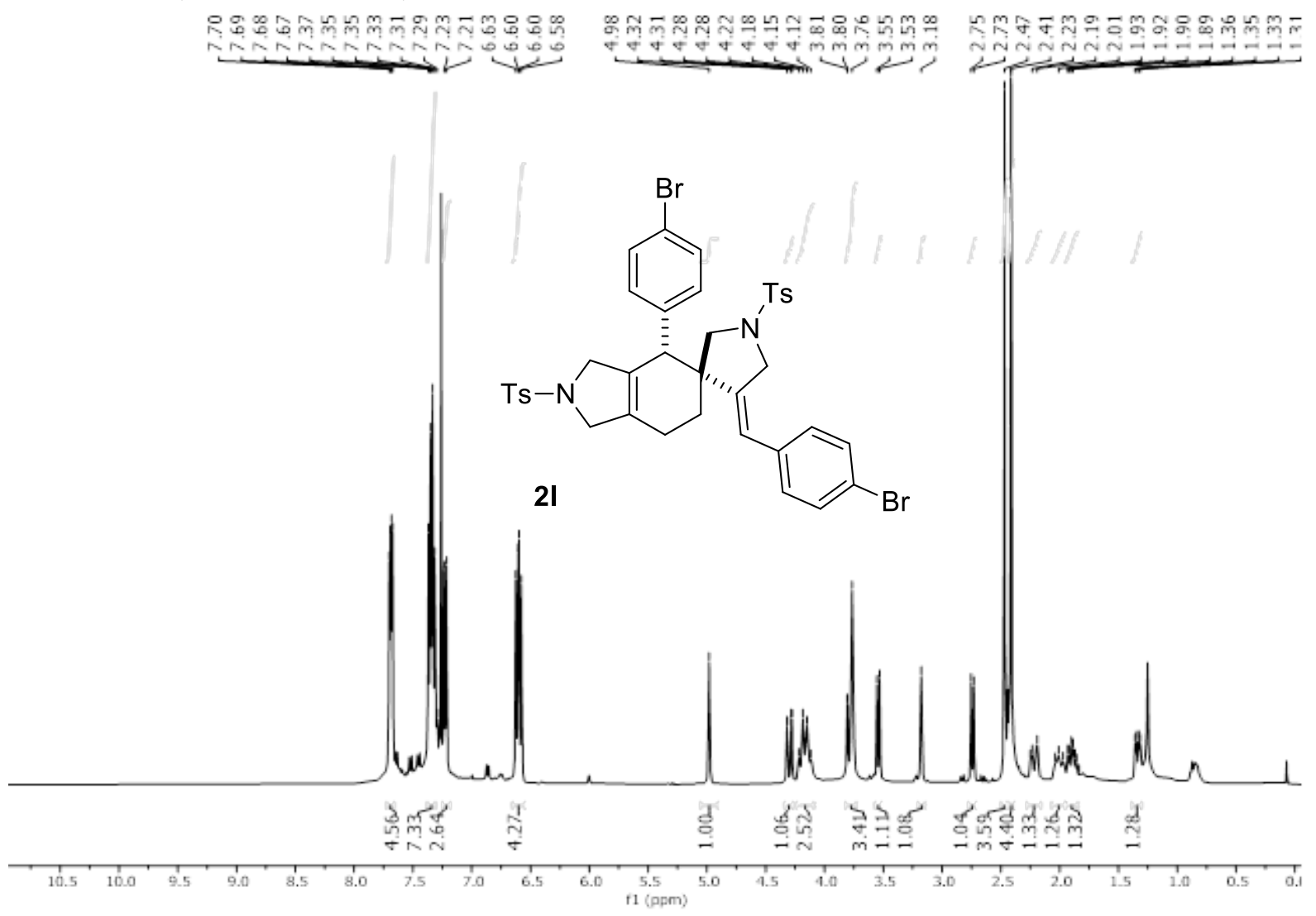

21, ${ }^{13} \mathbf{C}\{\mathbf{H}\}$ NMR $\left(101 \mathrm{MHz}, \mathrm{CDCl}_{3}\right)$

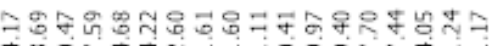

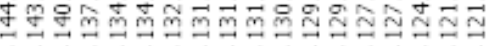
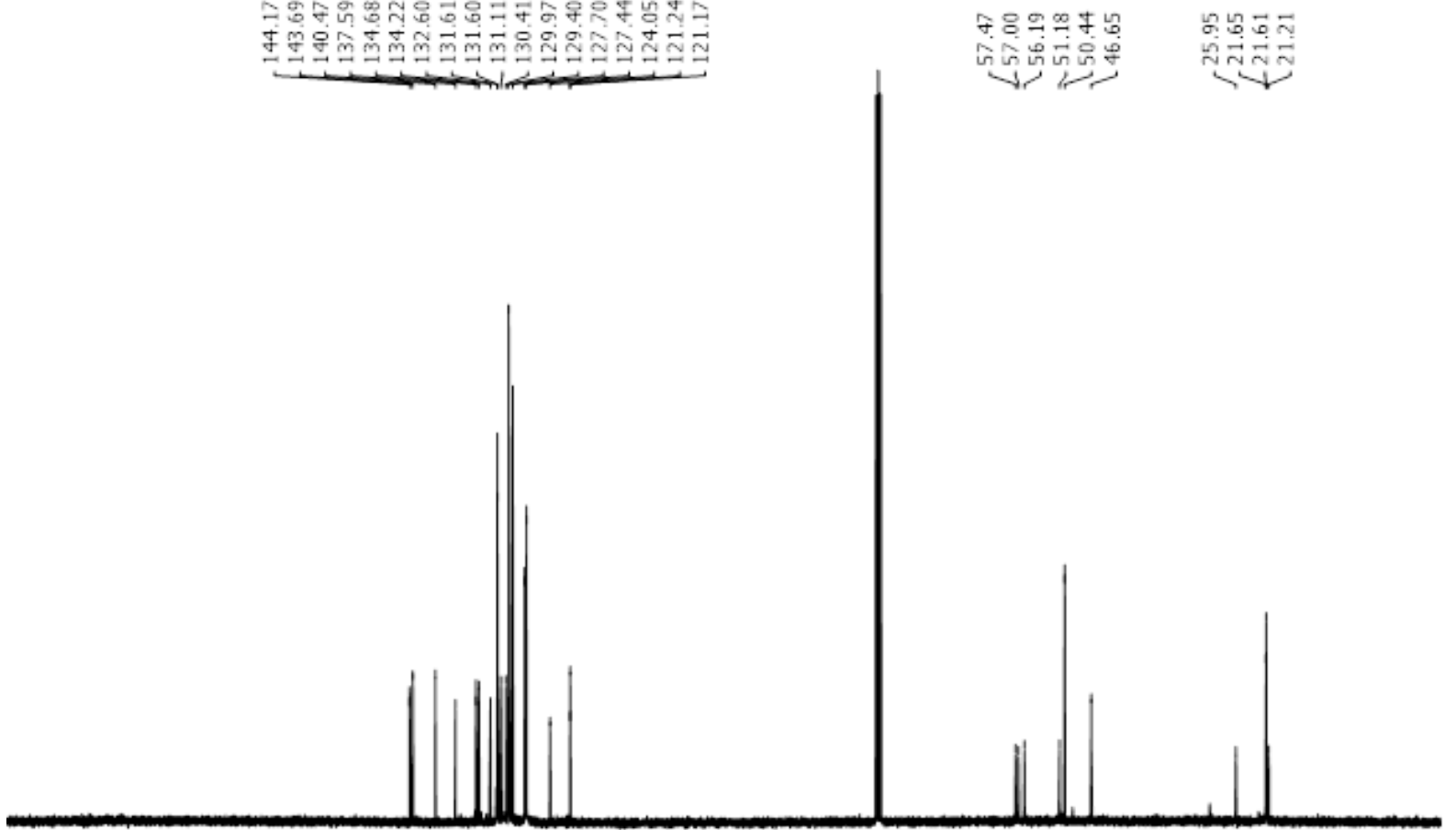

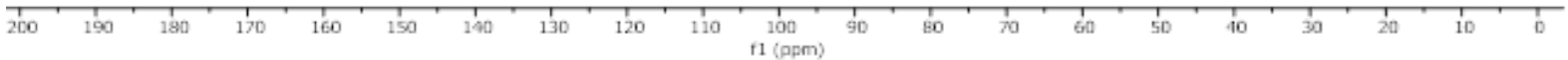


21, ${ }^{1} \mathbf{H}$ NMR (400 MHz, $\left.\mathrm{CDCl}_{3}\right)$

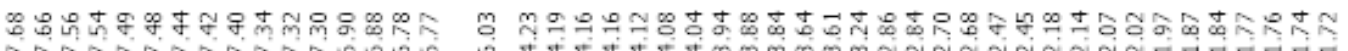

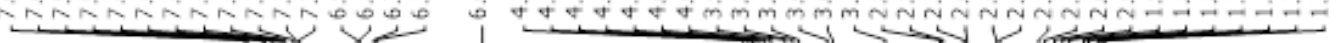

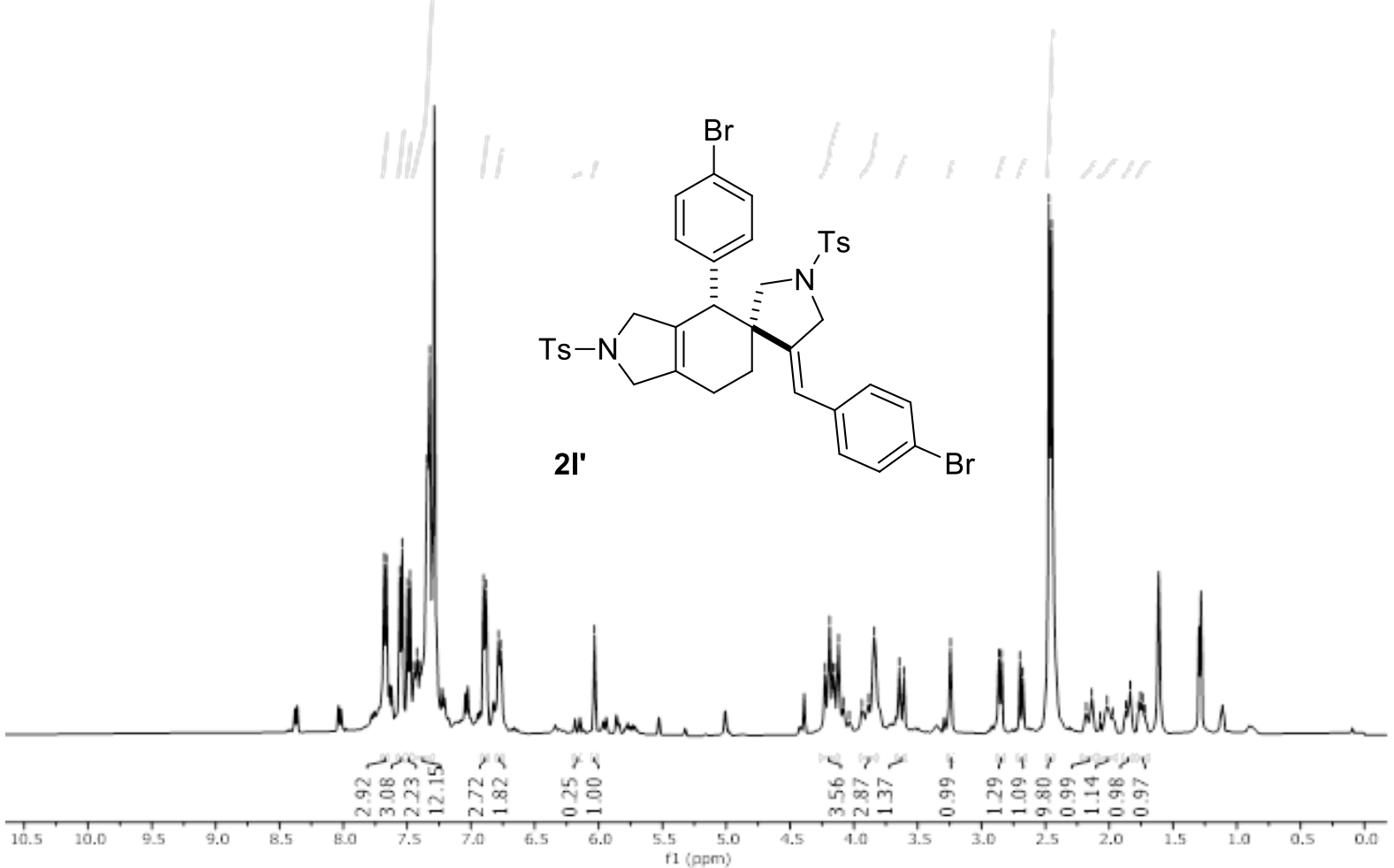

21', ${ }^{13} \mathbf{C}\{\mathbf{H}\}$ NMR $\left(101 \mathrm{MHz}, \mathrm{CDCl}_{3}\right)$

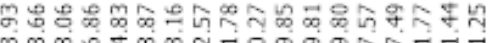

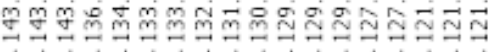

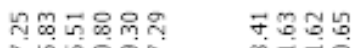

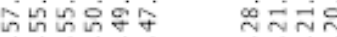

is is

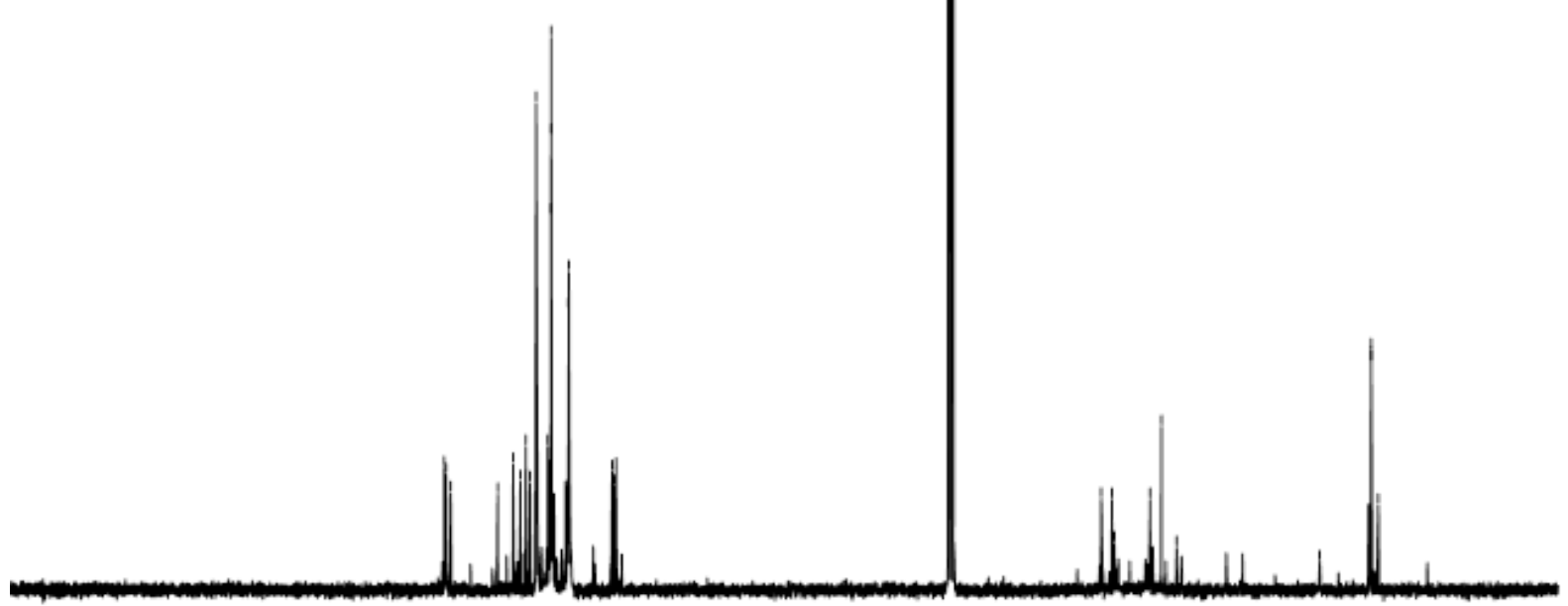

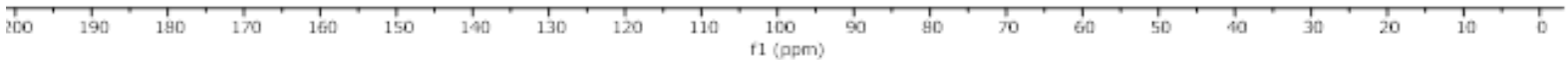


2m/2m', ${ }^{1} \mathbf{H}$ NMR $\left(400 \mathrm{MHz}, \mathrm{CDCl}_{3}\right)$

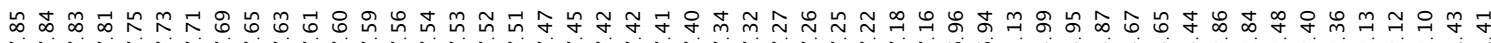

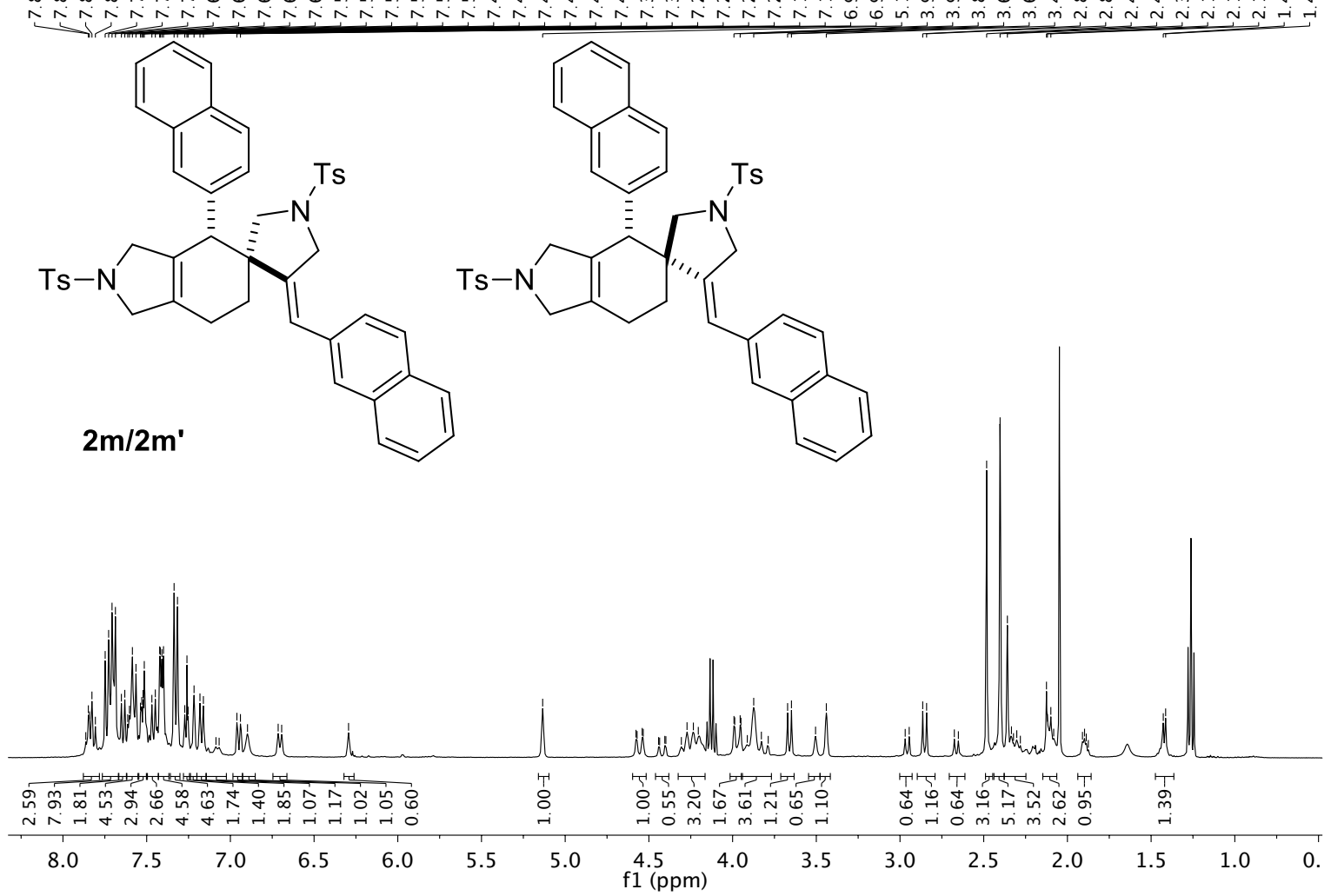

2m/2m, ${ }^{13} \mathbf{C}\{\mathbf{H}\} \mathbf{N M R}\left(101 \mathrm{MHz}, \mathrm{CDCl}_{3}\right)$

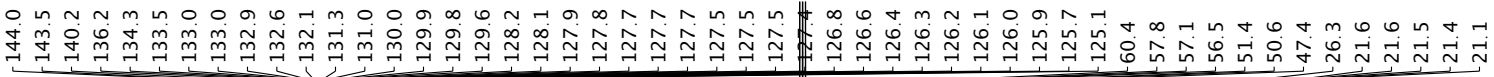

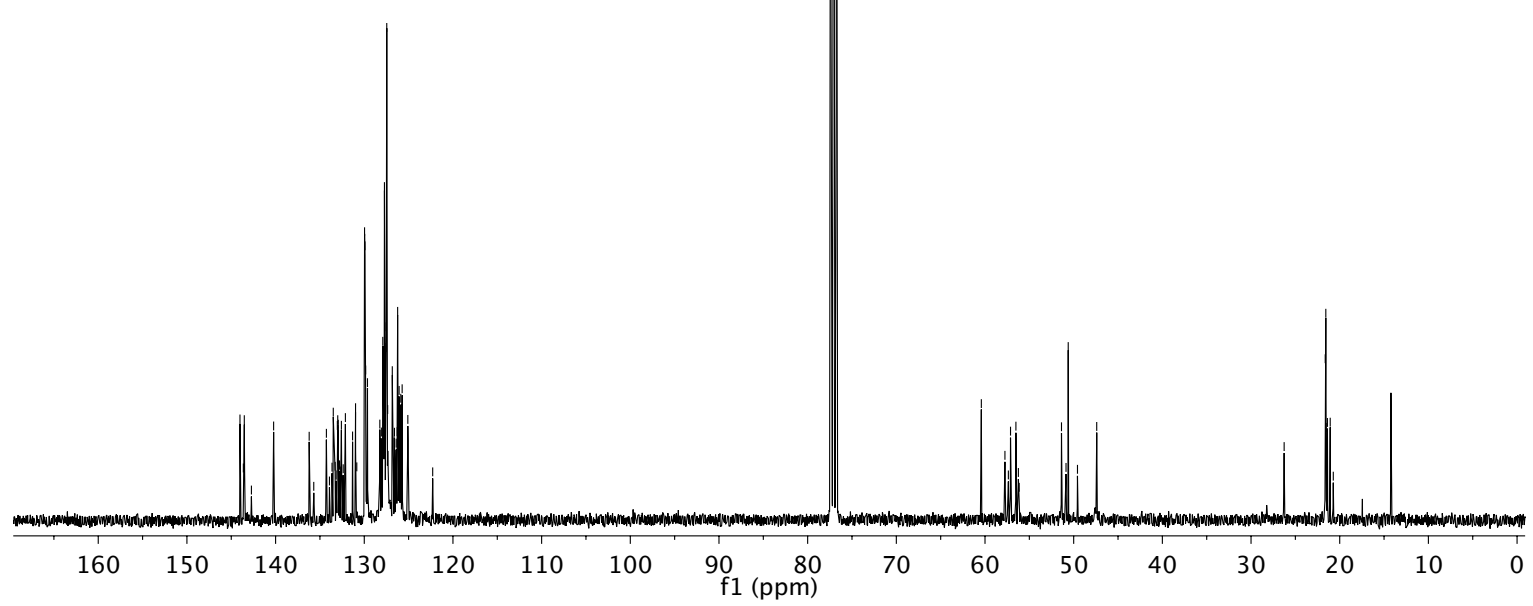


2n, ${ }^{1} \mathbf{H}$ NMR $\left(300 \mathrm{MHz}, \mathrm{CDCl}_{3}\right)$

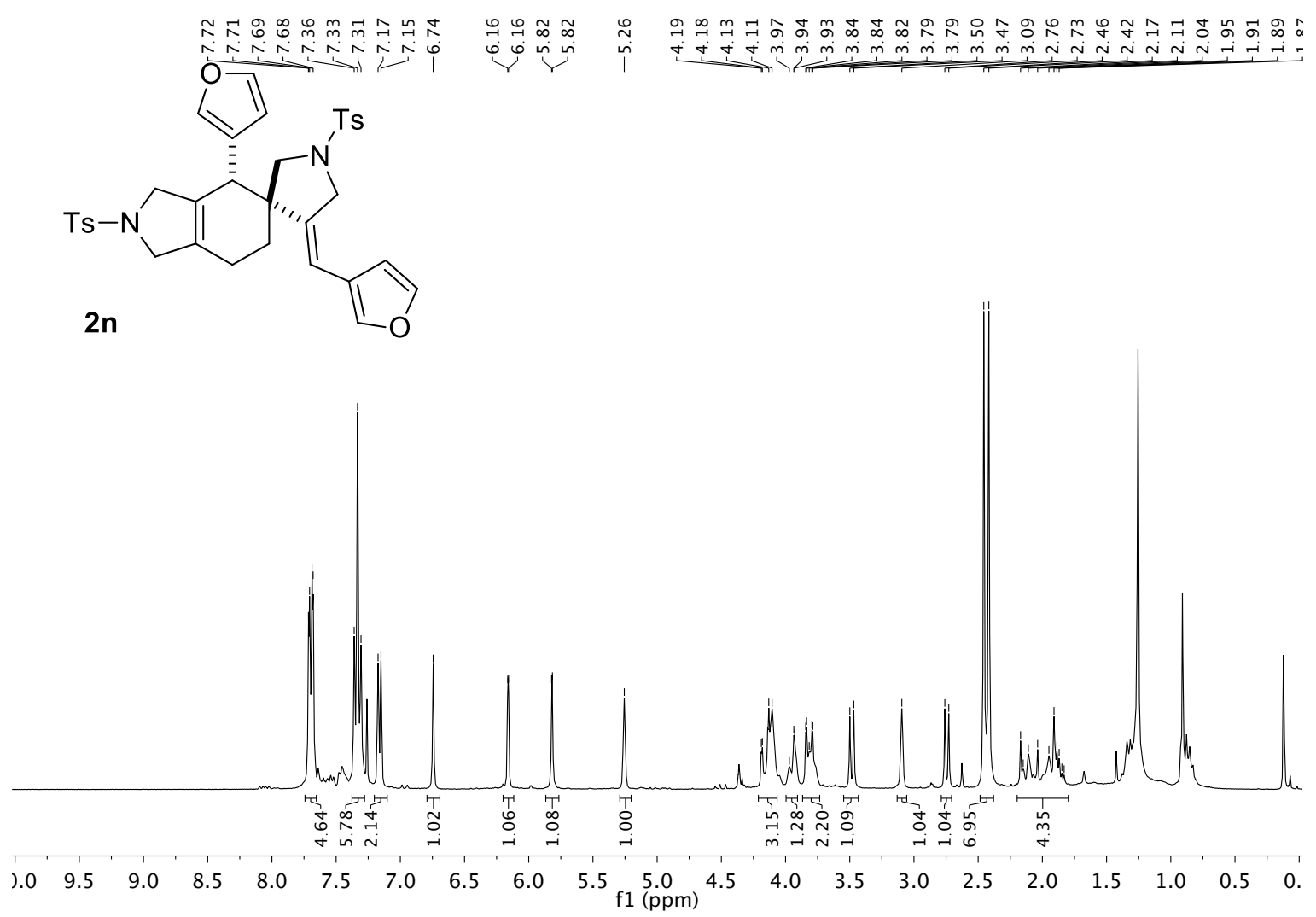

2n, ${ }^{13} \mathbf{C}\{\mathbf{H}\} \mathbf{N M R}\left(75 \mathrm{MHz}, \mathrm{CDCl}_{3}\right)$

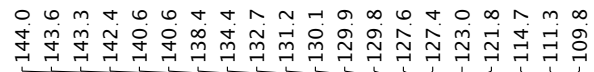

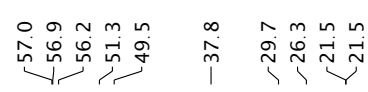

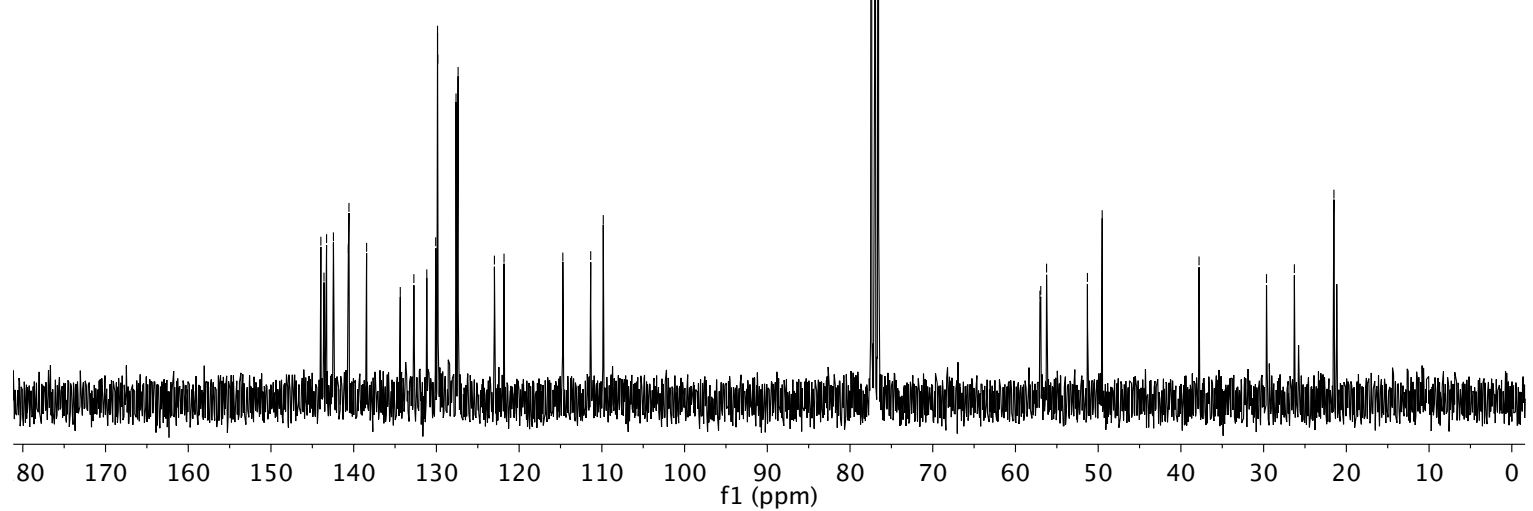


2n', ${ }^{1} \mathbf{H}$ NMR $\left(300 \mathrm{MHz}, \mathrm{CDCl}_{3}\right)$

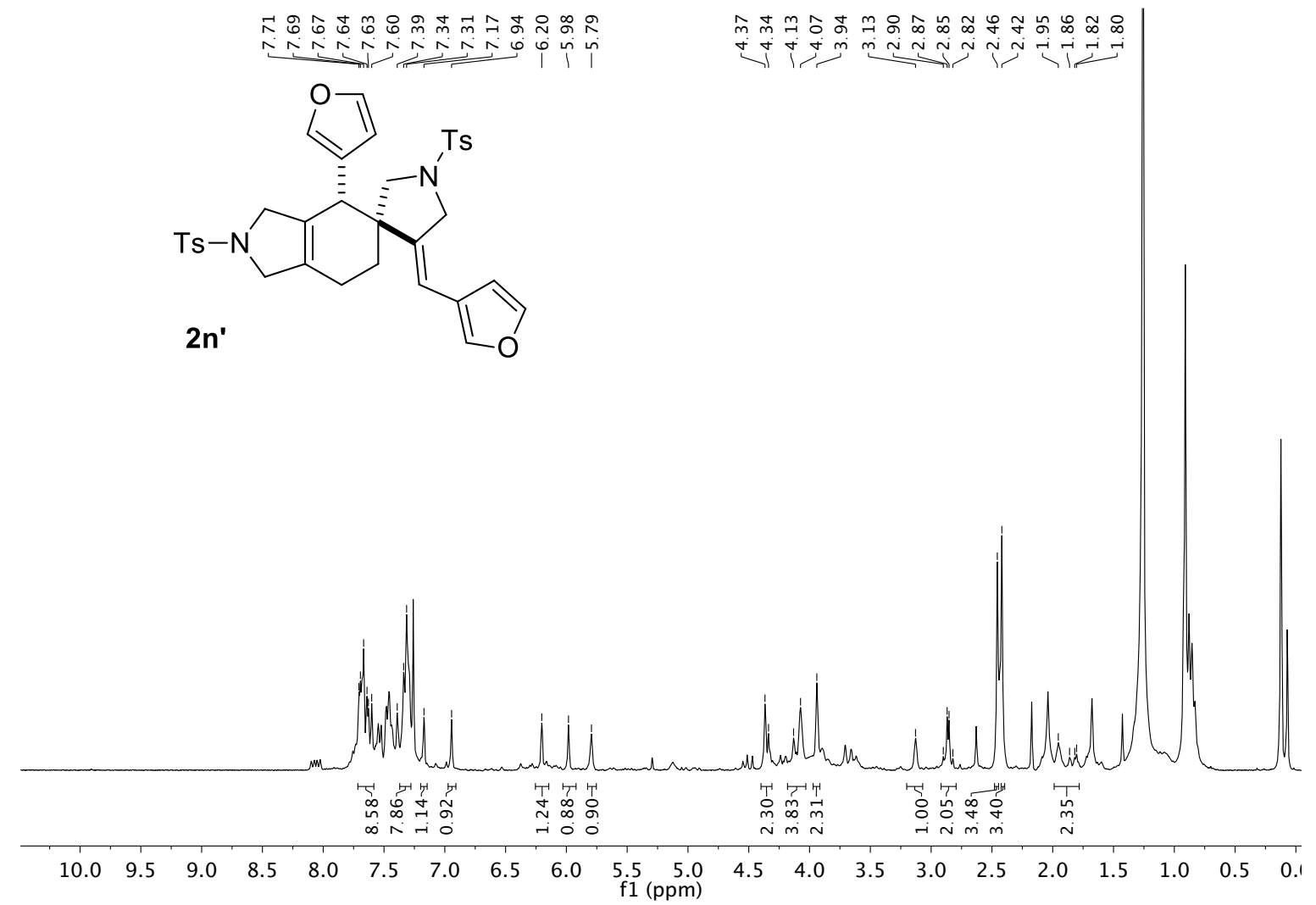

2n', ${ }^{13} \mathbf{C}\{\mathbf{H}\} \mathbf{N M R}\left(75 \mathrm{MHz}, \mathrm{CDCl}_{3}\right)$

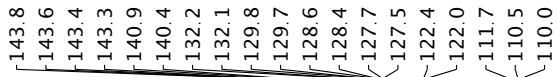

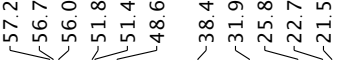

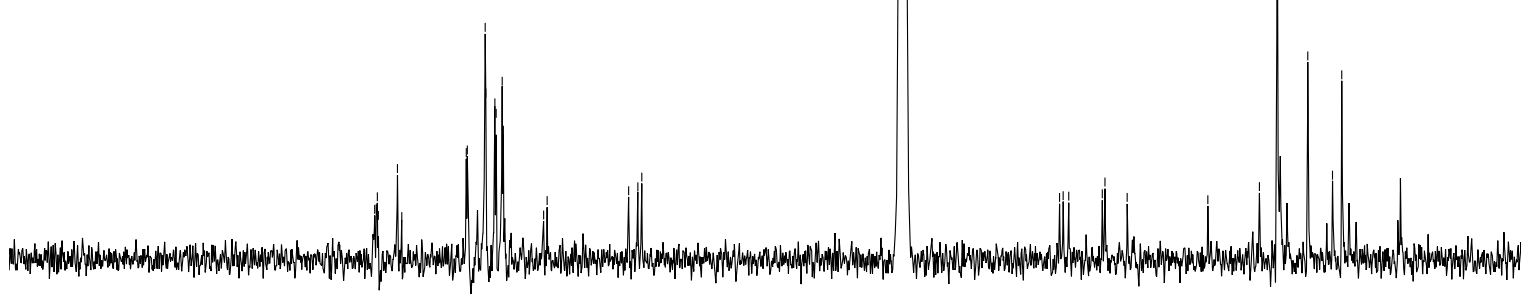

$\begin{array}{llllllllll}30 & 180 & 170 & 160 & 150 & 140 & 130 & 120 & 110 & 100 \\ \mathrm{f} 1(\mathrm{ppm}) & 90\end{array}$ 
2o, ${ }^{1} \mathbf{H}$ NMR $\left(400 \mathrm{MHz}, \mathrm{CDCl}_{3}\right)$

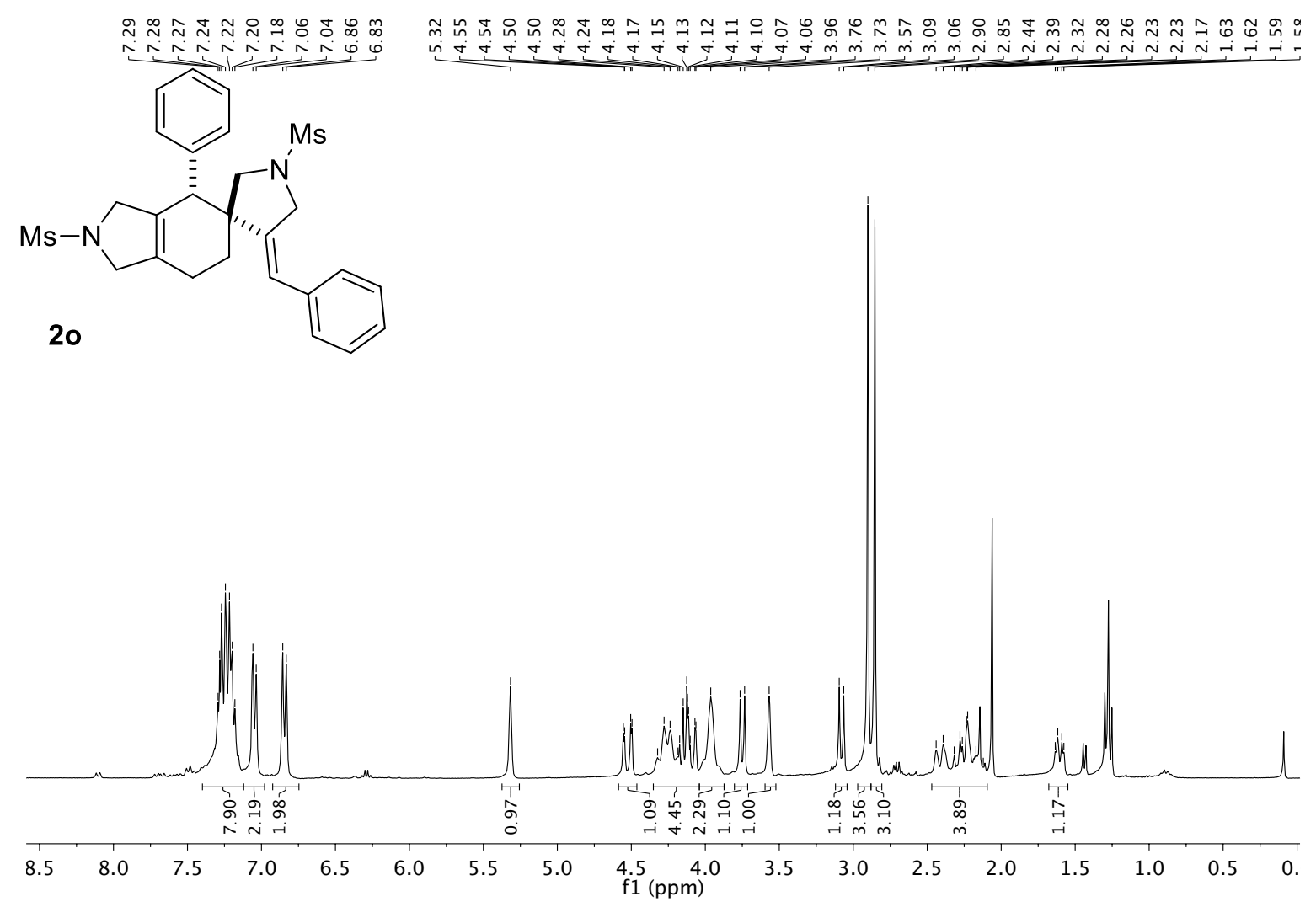

2o, ${ }^{13} \mathbf{C}\{\mathbf{H}\}$ NMR $\left(101 \mathrm{MHz}, \mathrm{CDCl}_{3}\right)$

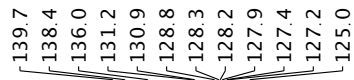

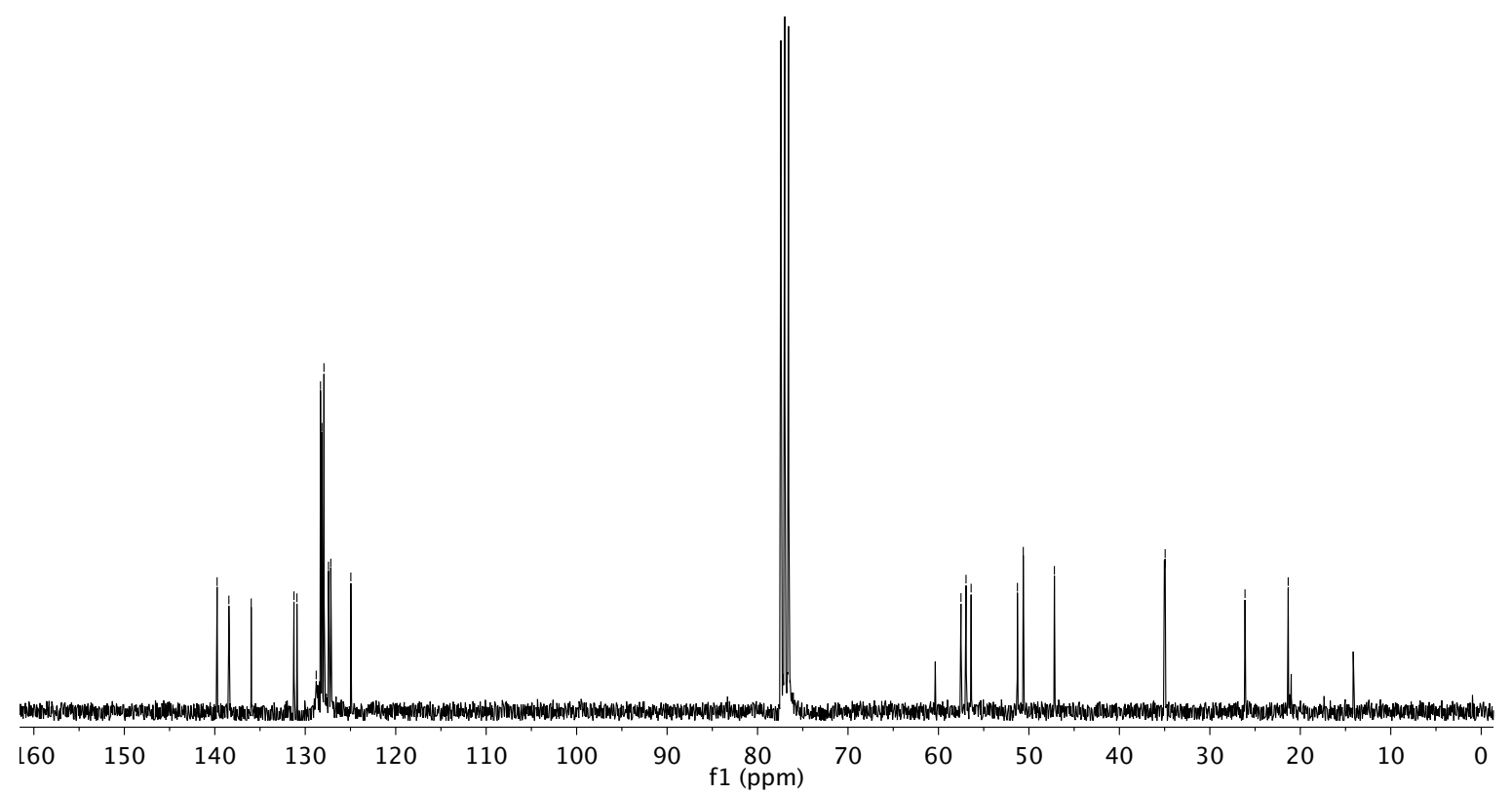


2p/2p', ${ }^{1} \mathbf{H}$ NMR $\left(400 \mathrm{MHz}, \mathrm{CDCl}_{3}\right)$

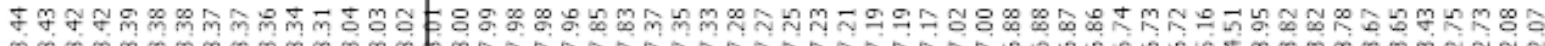

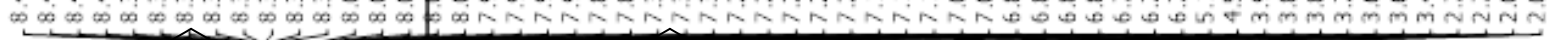

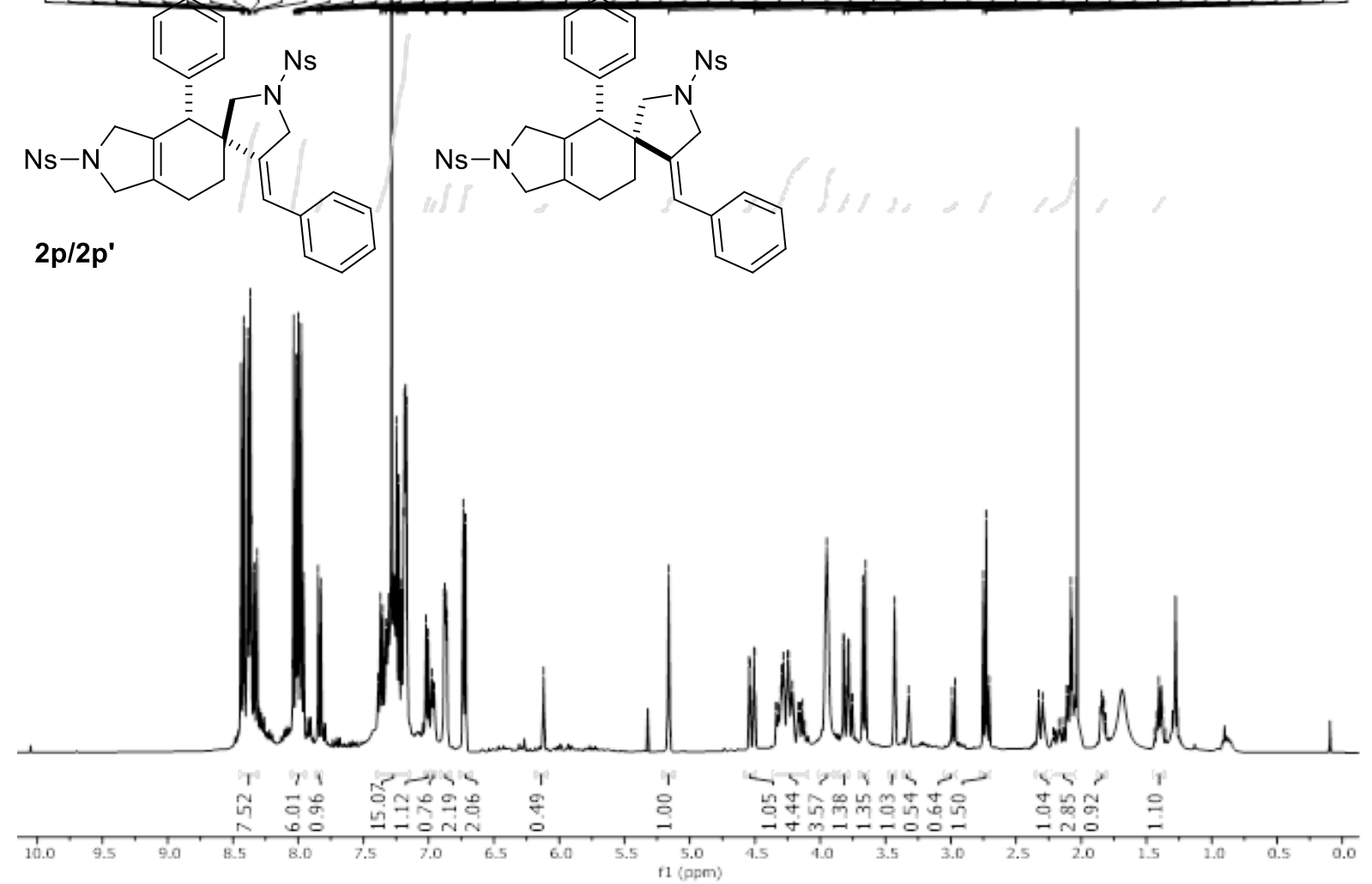

2p/2p, ${ }^{13} \mathbf{C}\{\mathbf{H}\}$ NMR $\left(101 \mathrm{MHz}, \mathrm{CDCl}_{3}\right)$

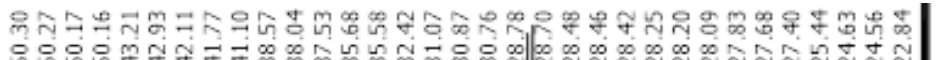

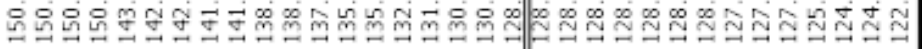

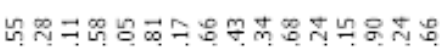

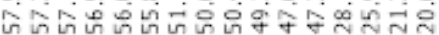

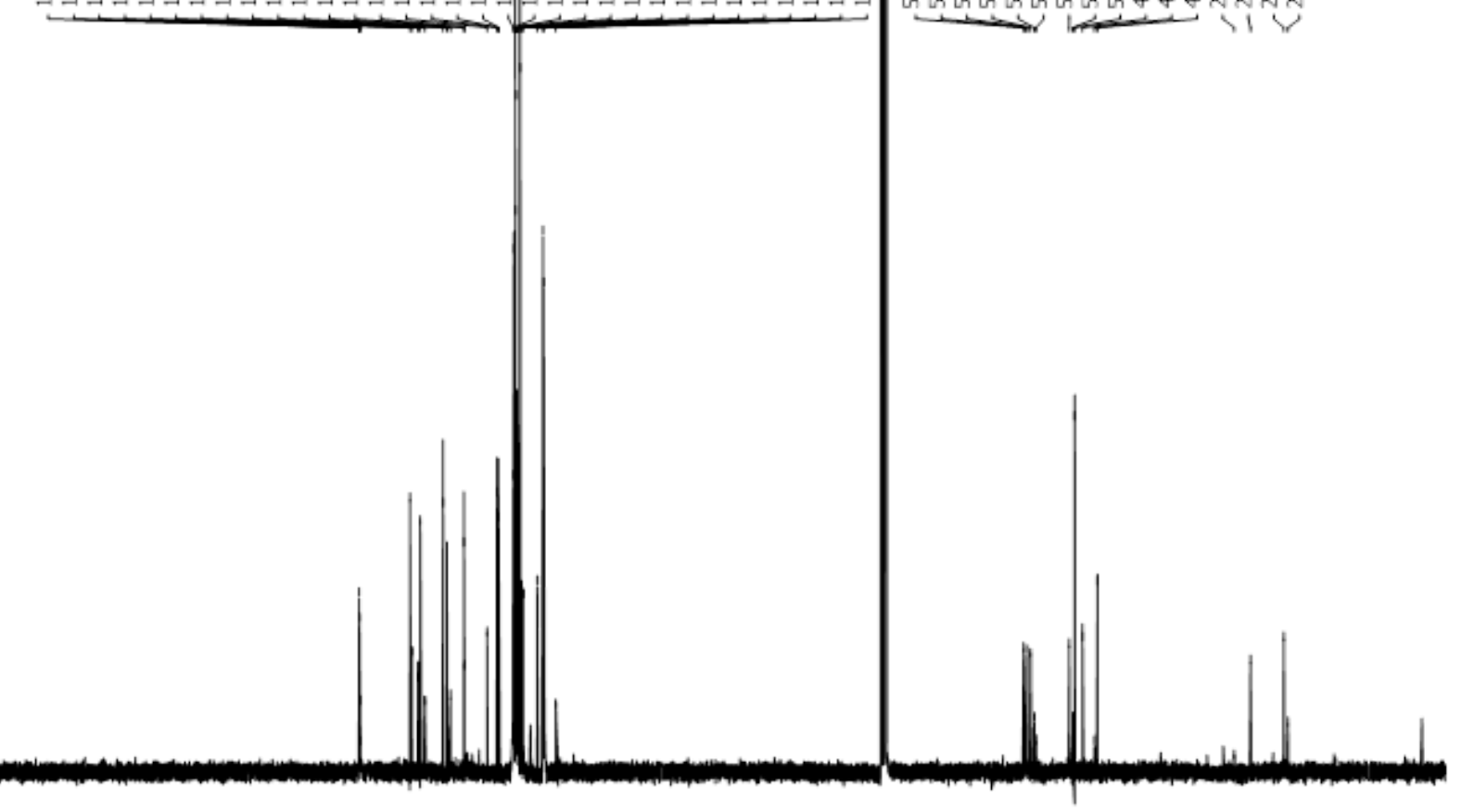

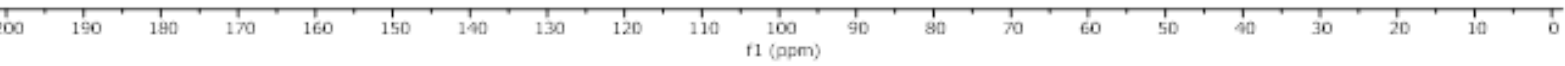


2q, ${ }^{1} \mathbf{H}$ NMR (400 MHz, $\left.\mathrm{CDCl}_{3}\right)$

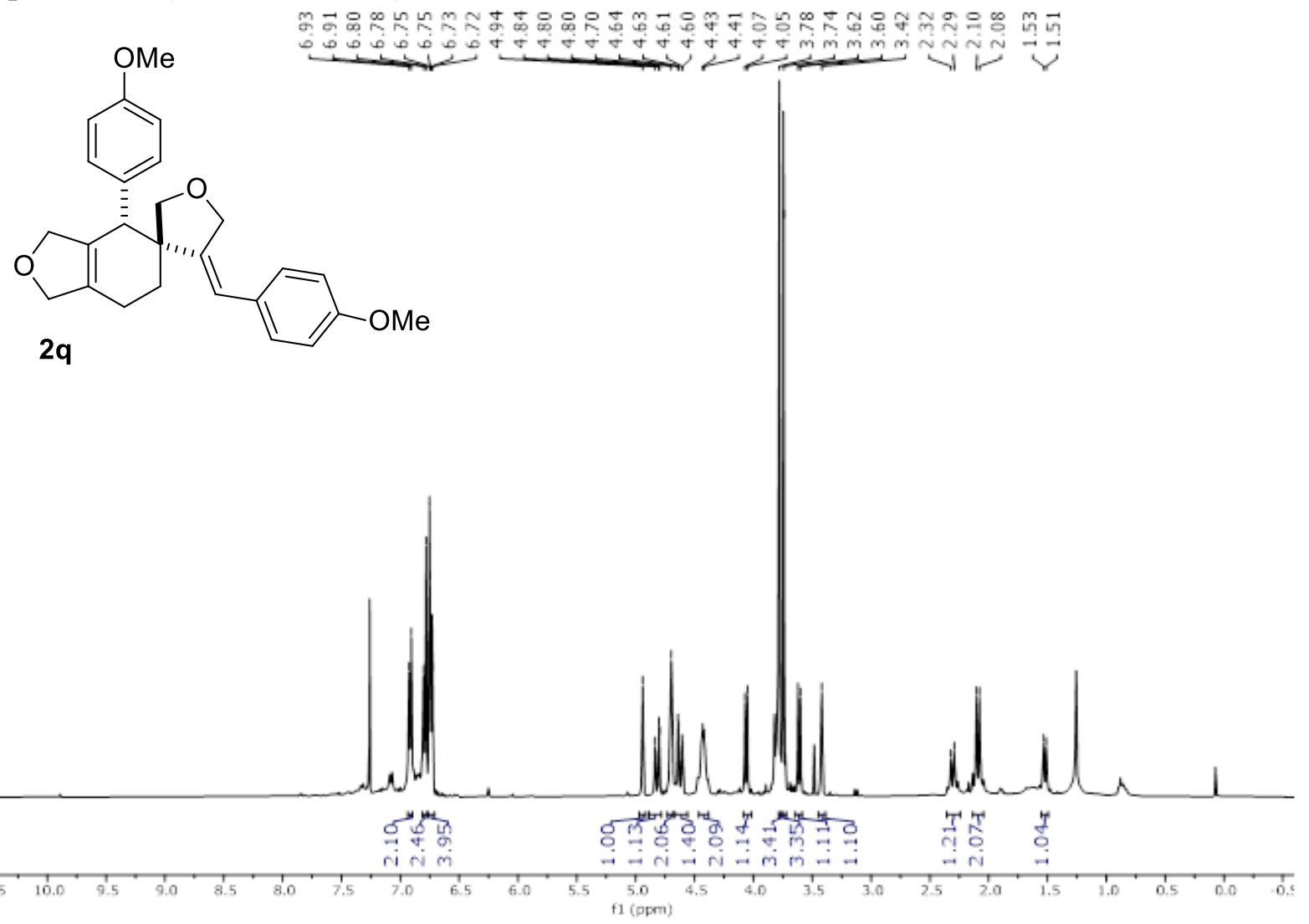

2q, ${ }^{13} \mathbf{C}\{\mathbf{H}\}$ NMR (101 MHz, $\left.\mathrm{CDCl}_{3}\right)$

年
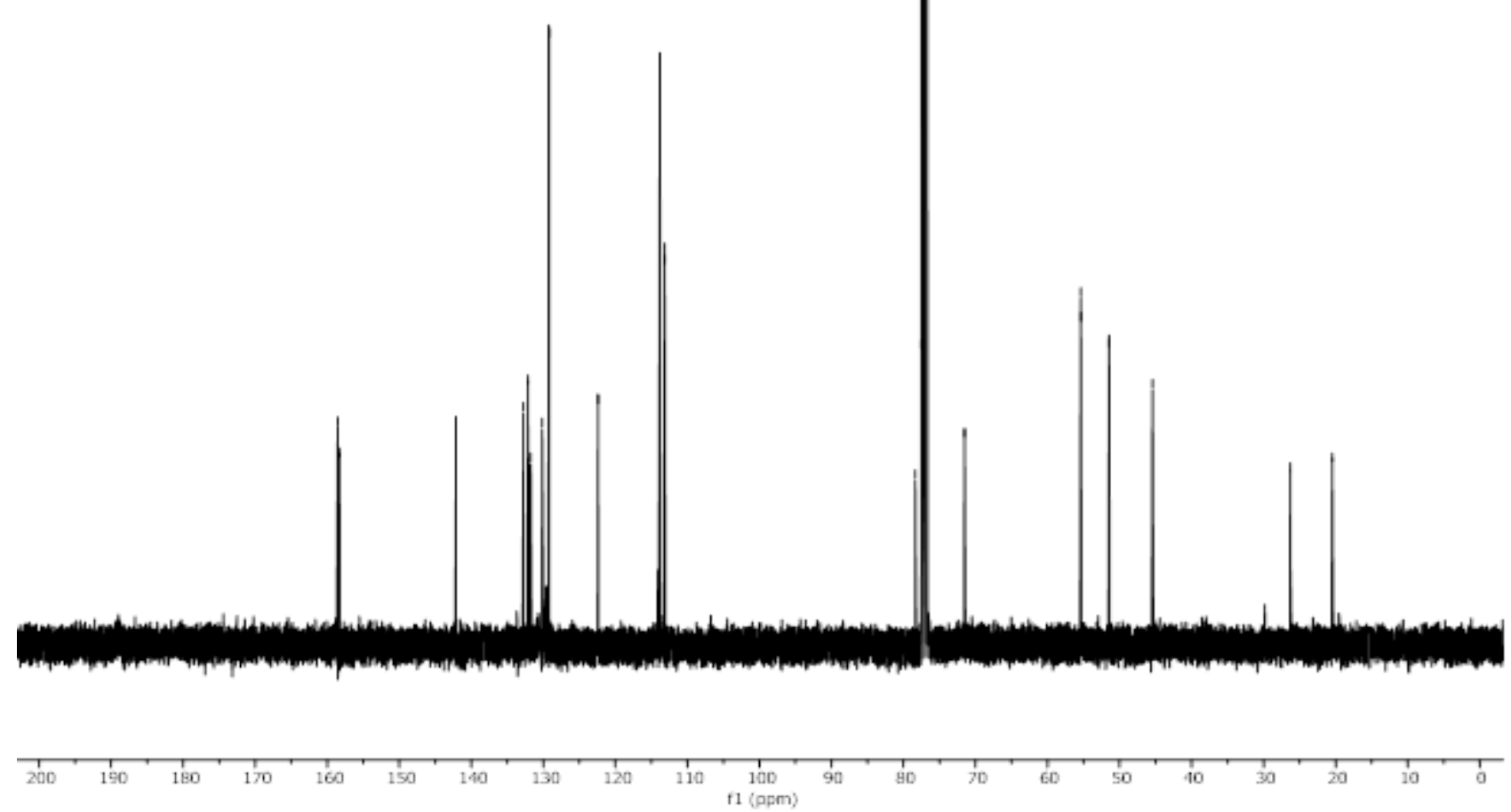
2q', ${ }^{1} \mathbf{H}$ NMR (400 MHz, $\left.\mathrm{CDCl}_{3}\right)$

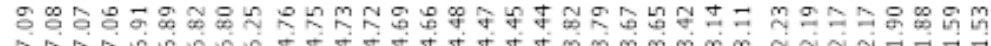

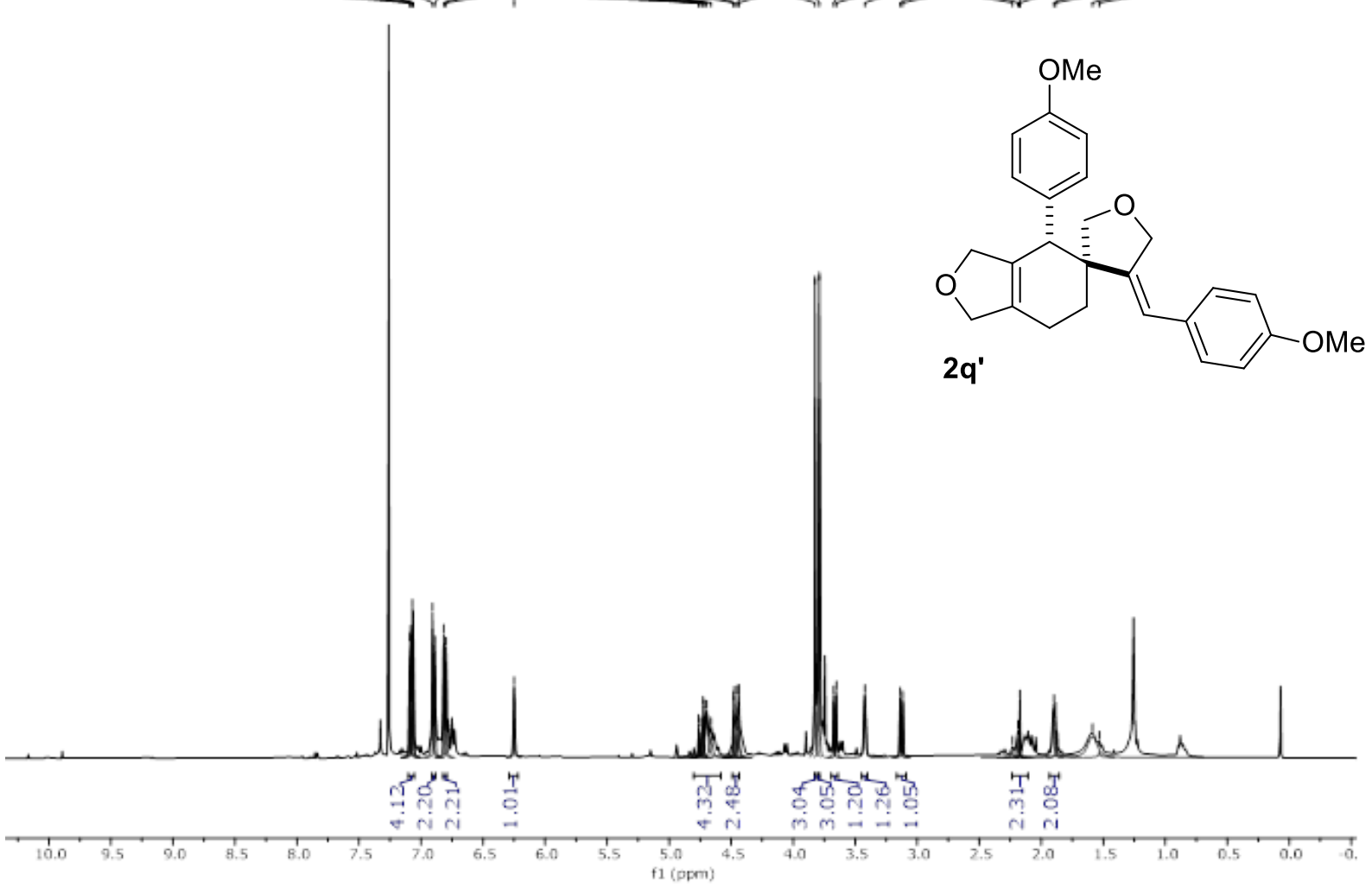

2q', ${ }^{13} \mathbf{C}\{\mathbf{H}\}$ NMR $\left(101 \mathrm{MHz}, \mathrm{CDCl}_{3}\right)$

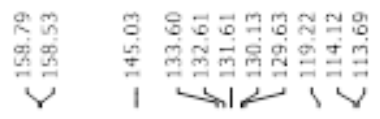$$
\text { | }
$$

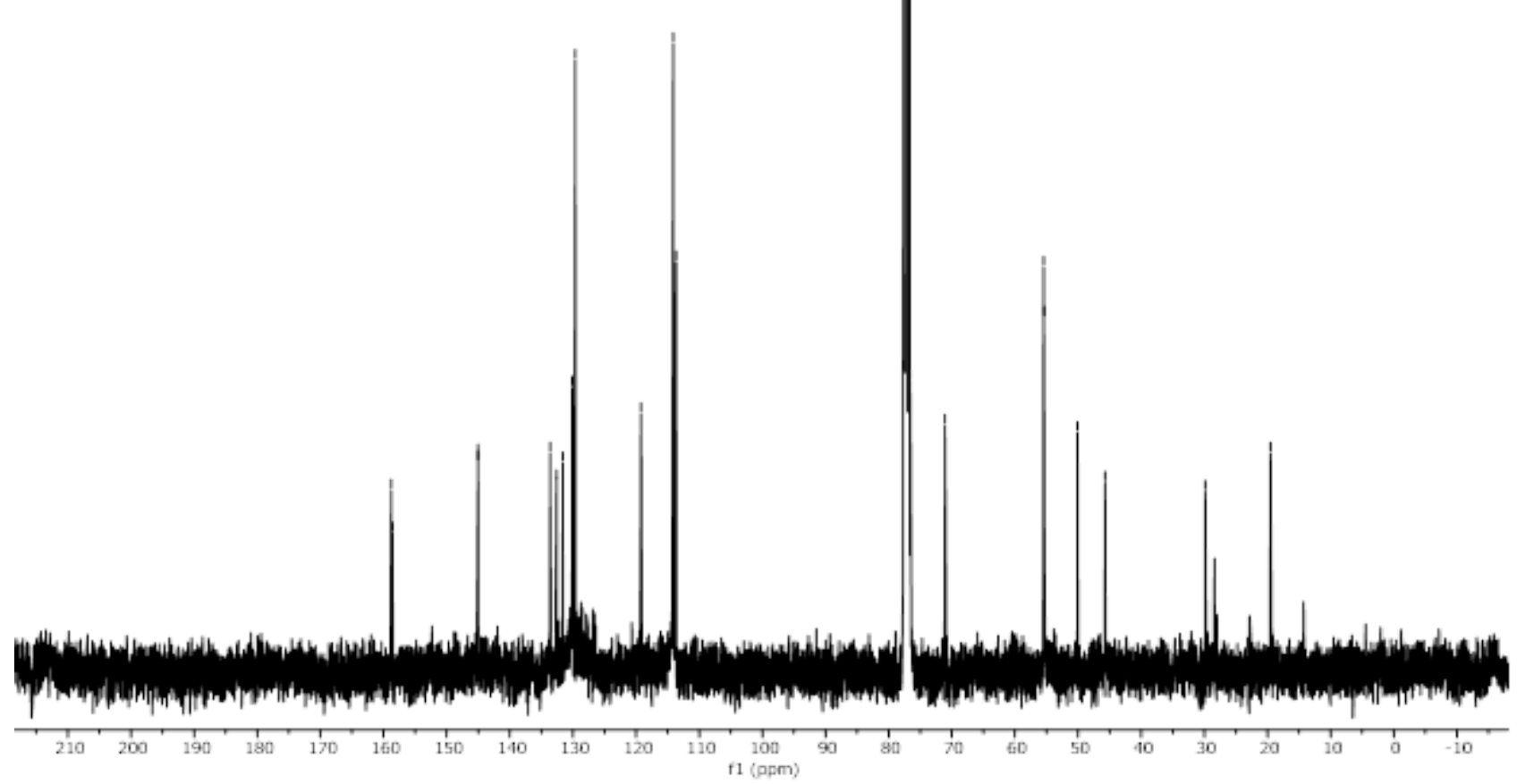


2r, ${ }^{1} \mathbf{H}$ NMR $\left(400 \mathrm{MHz}, \mathrm{CDCl}_{3}\right)$

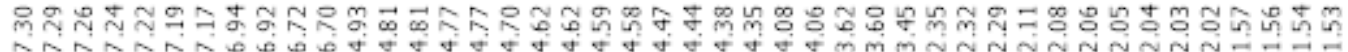

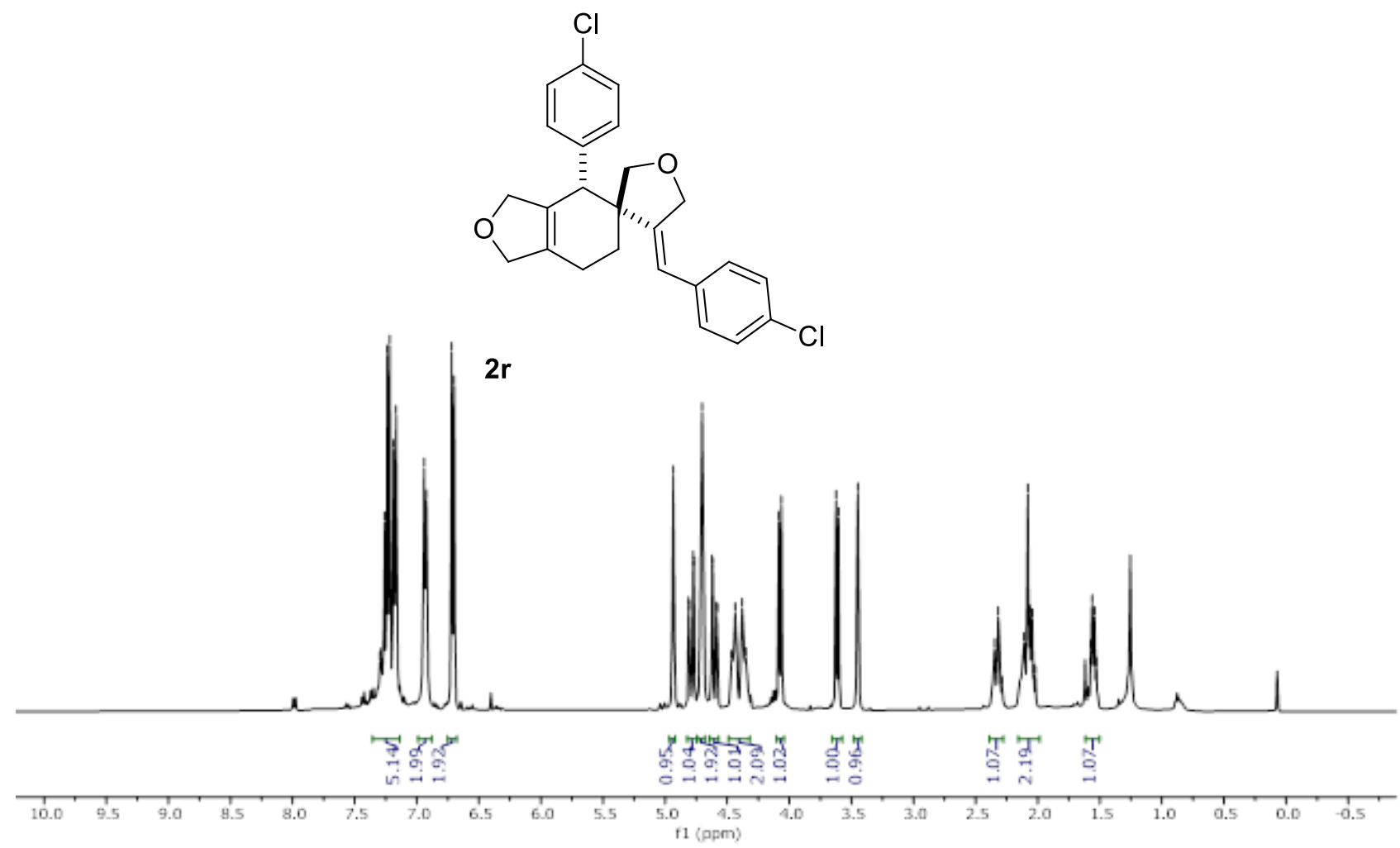

2r, ${ }^{13} \mathbf{C}\{\mathbf{H}\}$ NMR $\left(101 \mathrm{MHz}, \mathrm{CDCl}_{3}\right)$

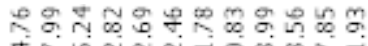

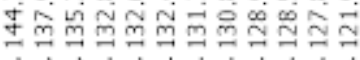

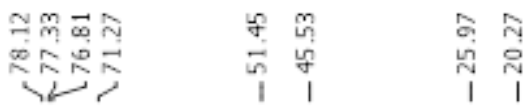
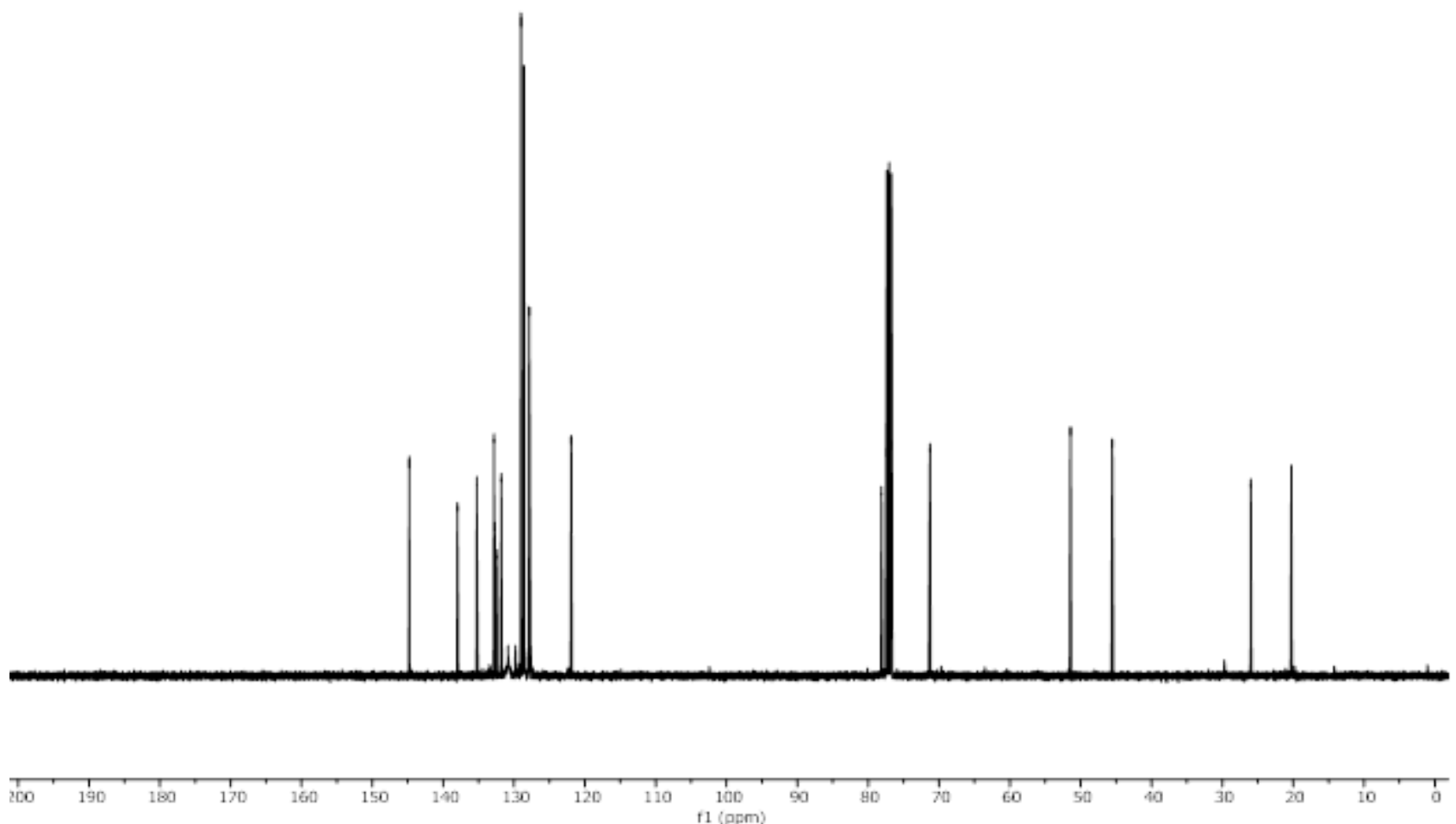
2r', ${ }^{1} \mathbf{H}$ NMR (400 MHz, $\left.\mathrm{CDCl}_{3}\right)$

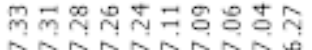

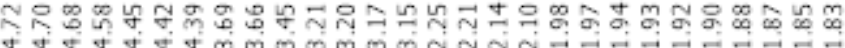

$\rightarrow$

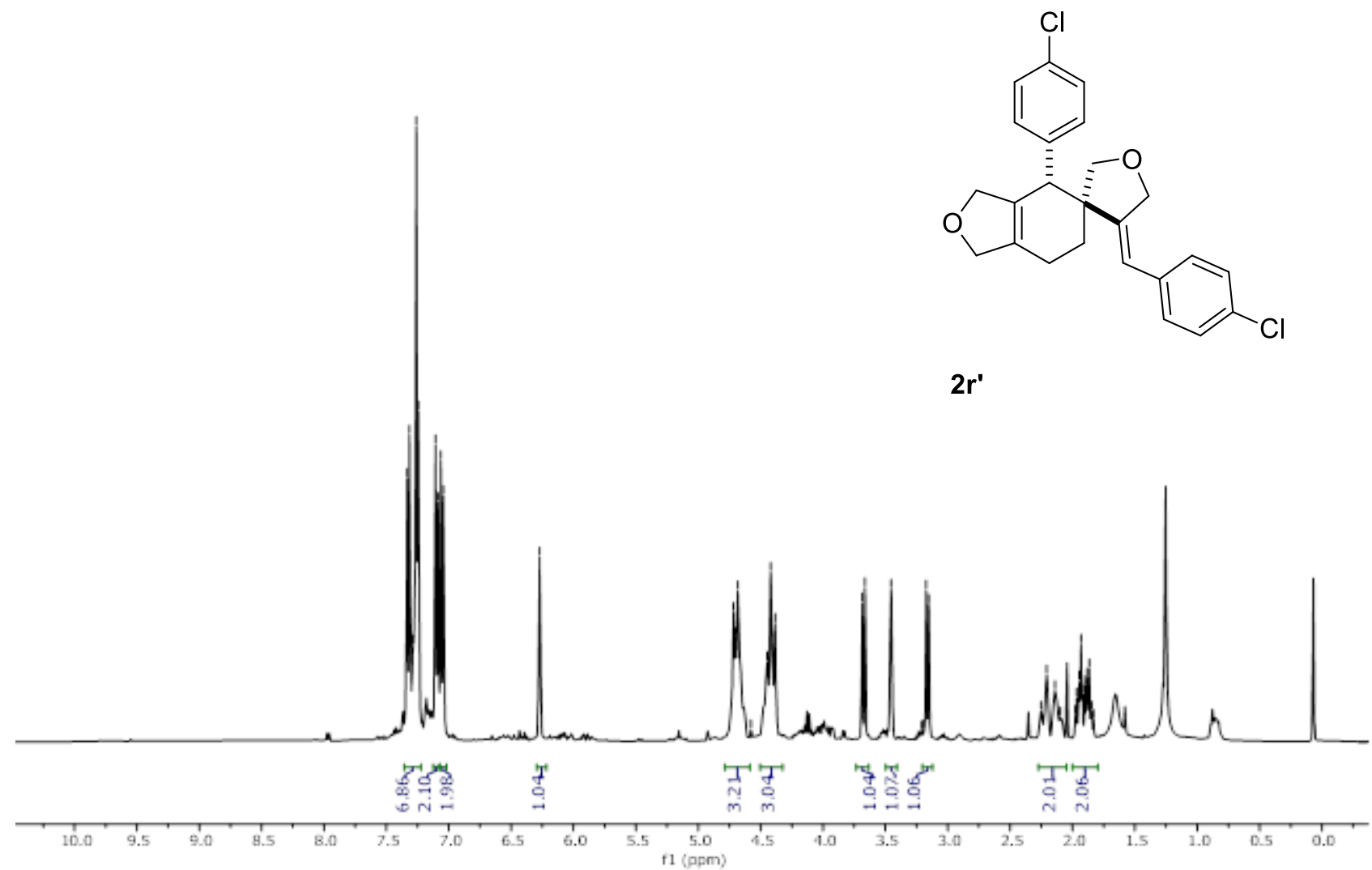

$2 r^{\prime}$

2r', ${ }^{13} \mathbf{C}\{\mathbf{H}\}$ NMR $\left(101 \mathrm{MHz}, \mathrm{CDCl}_{3}\right)$

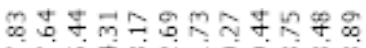

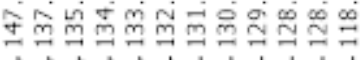

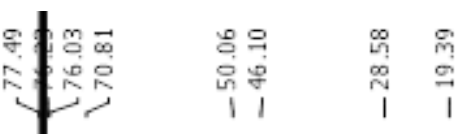

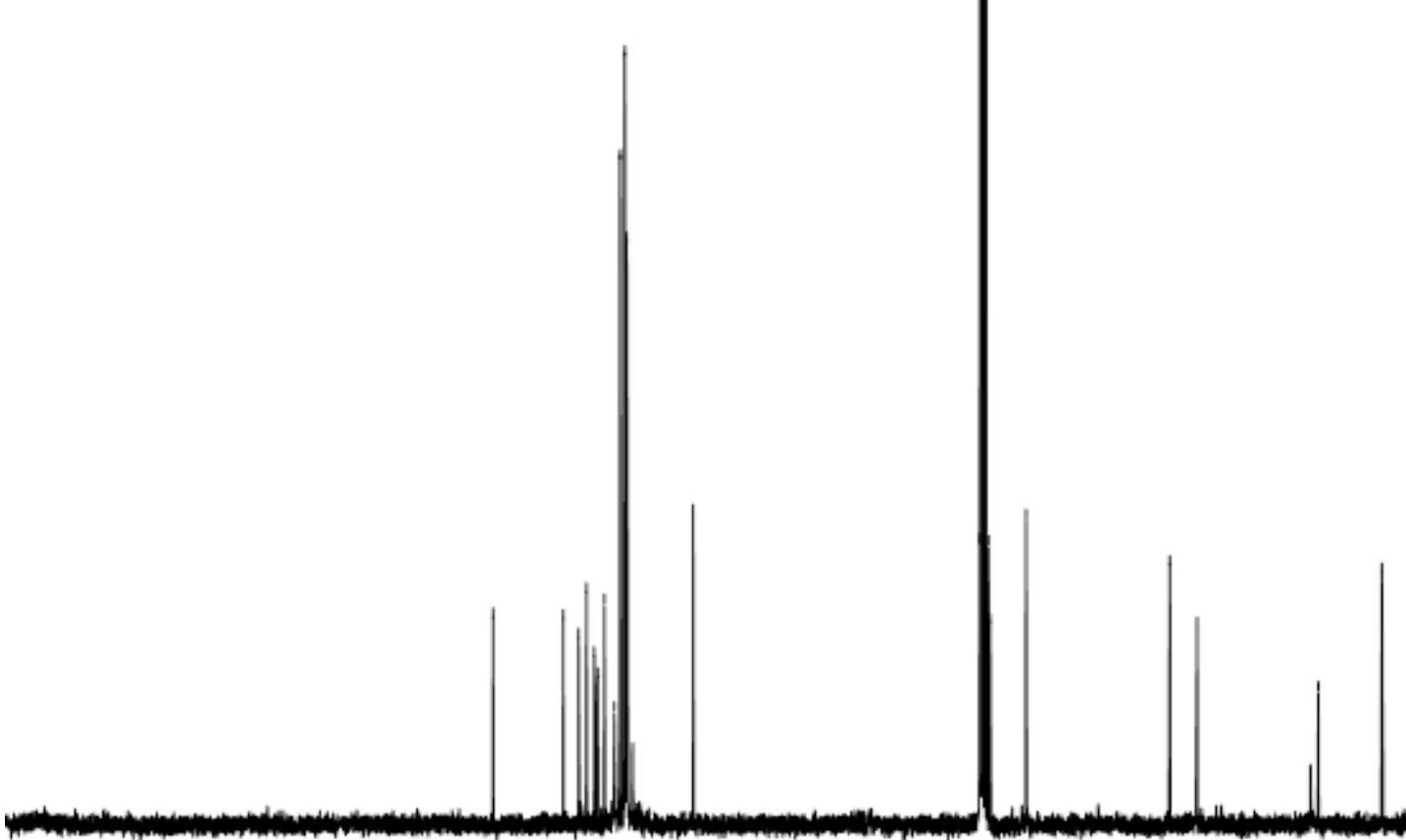

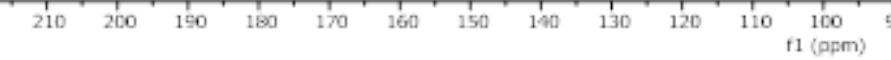


2s, ${ }^{1} \mathbf{H}$ NMR $\left(400 \mathrm{MHz}, \mathrm{CDCl}_{3}\right)$

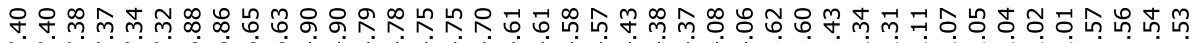

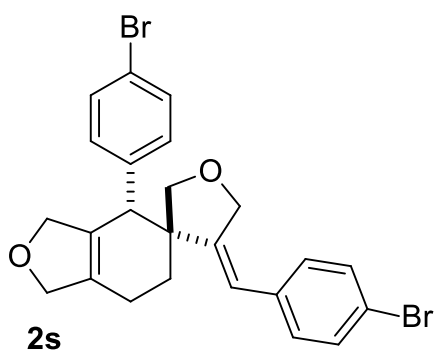

$$
\mathrm{Br}
$$

1.

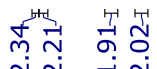

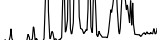

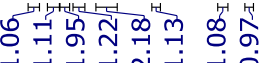

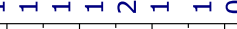

2s, ${ }^{13} \mathbf{C}\{\mathbf{H}\}$ NMR $\left(101 \mathrm{MHz}, \mathrm{CDCl}_{3}\right)$

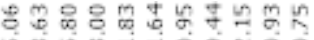

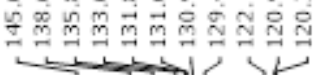

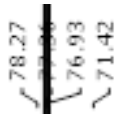

की

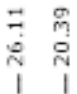

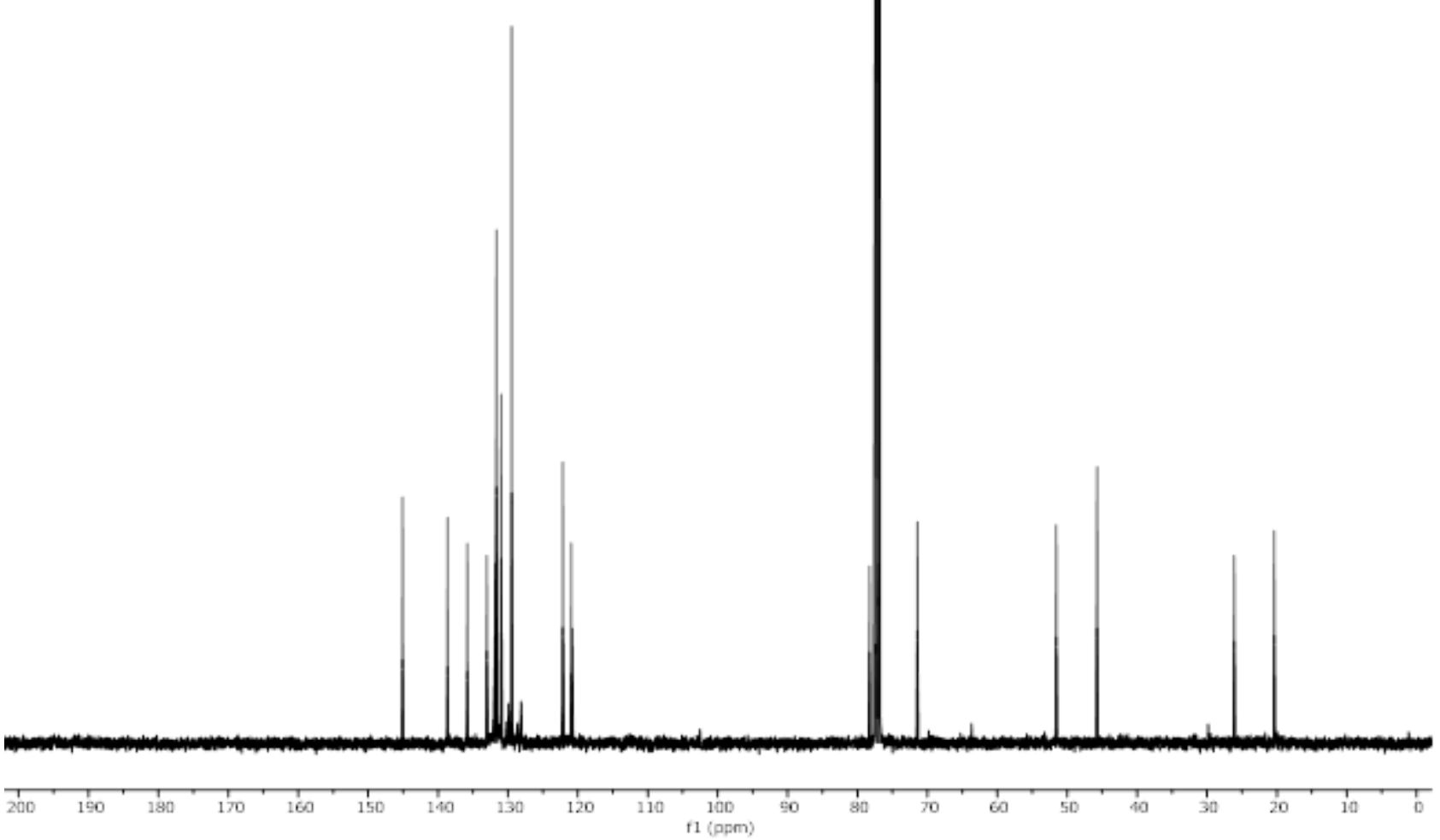


2t, ${ }^{1} \mathbf{H}$ NMR $\left(400 \mathrm{MHz}, \mathrm{CDCl}_{3}\right)$

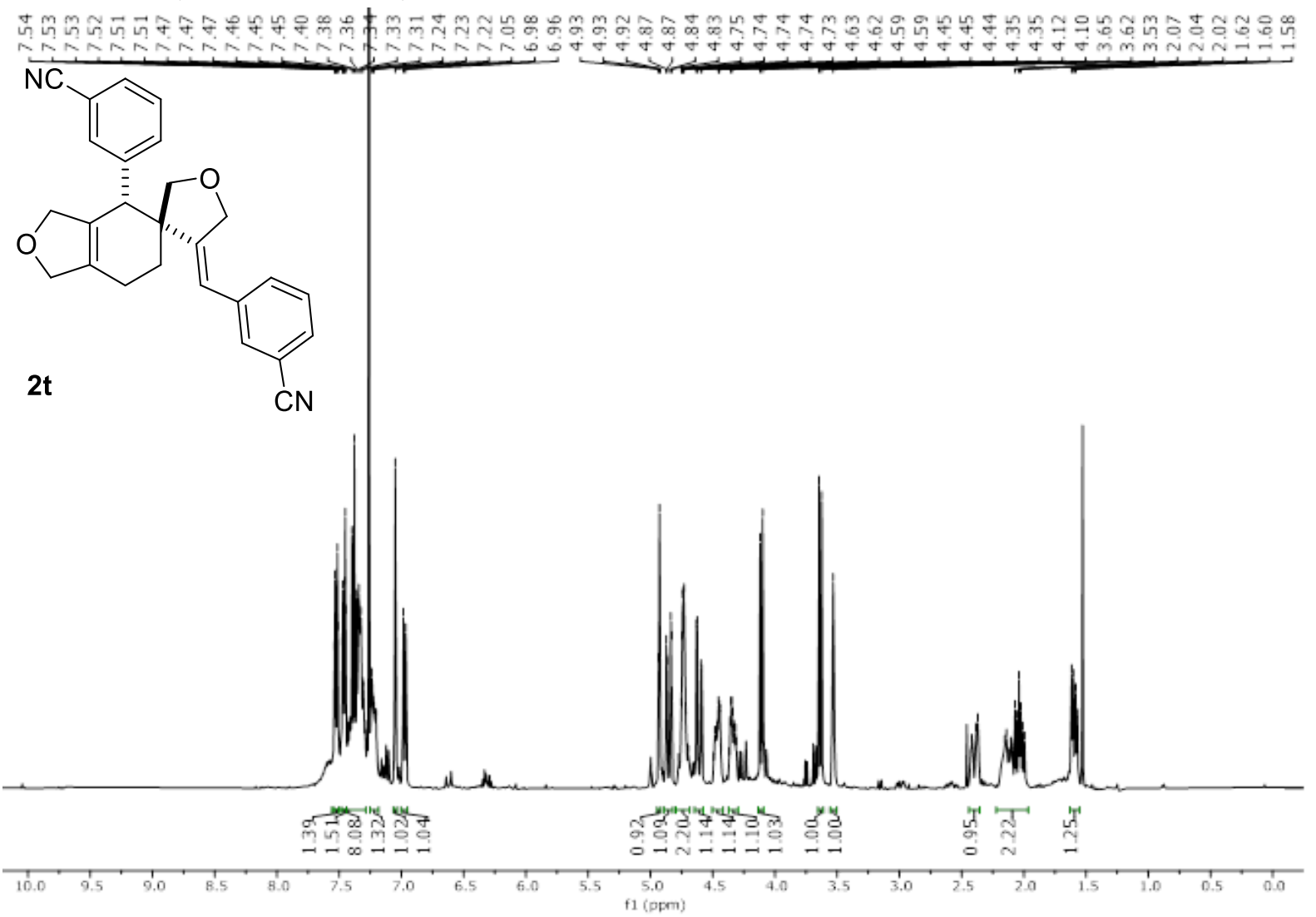

2t, ${ }^{13} \mathbf{C}\{\mathbf{H}\}$ NMR $\left(101 \mathrm{MHz}, \mathrm{CDCl}_{3}\right)$

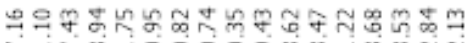

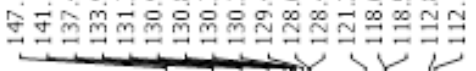

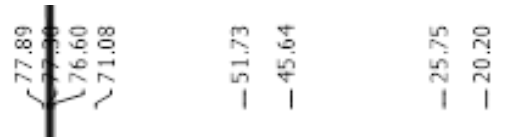
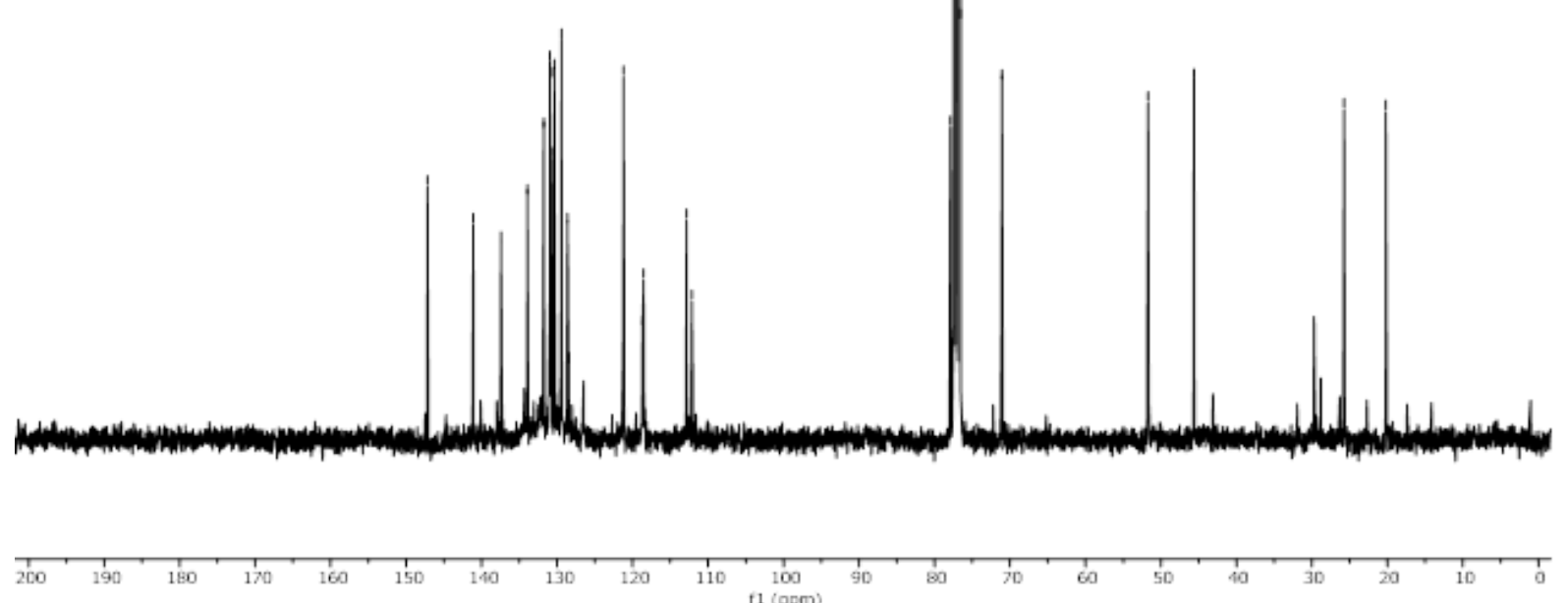
2t', ${ }^{1} \mathbf{H}$ NMR $\left(400 \mathrm{MHz}, \mathrm{CDCl}_{3}\right)$

ํํำ

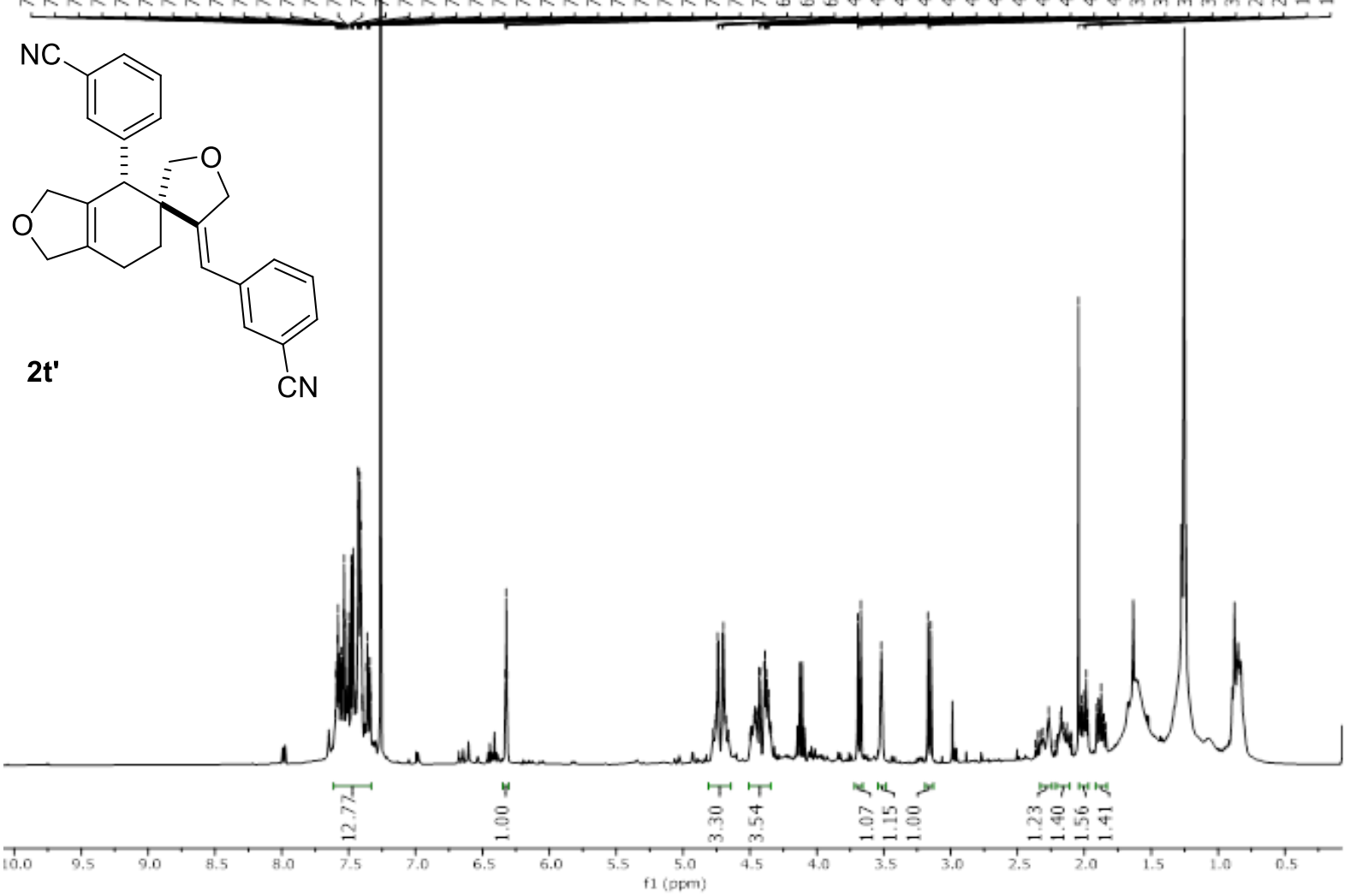

2t', ${ }^{13} \mathbf{C}\{\mathbf{H}\}$ NMR (101 MHz, $\left.\mathrm{CDCl}_{3}\right)$

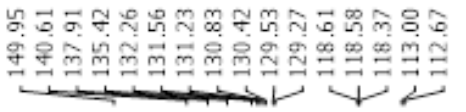

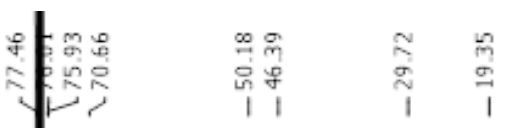
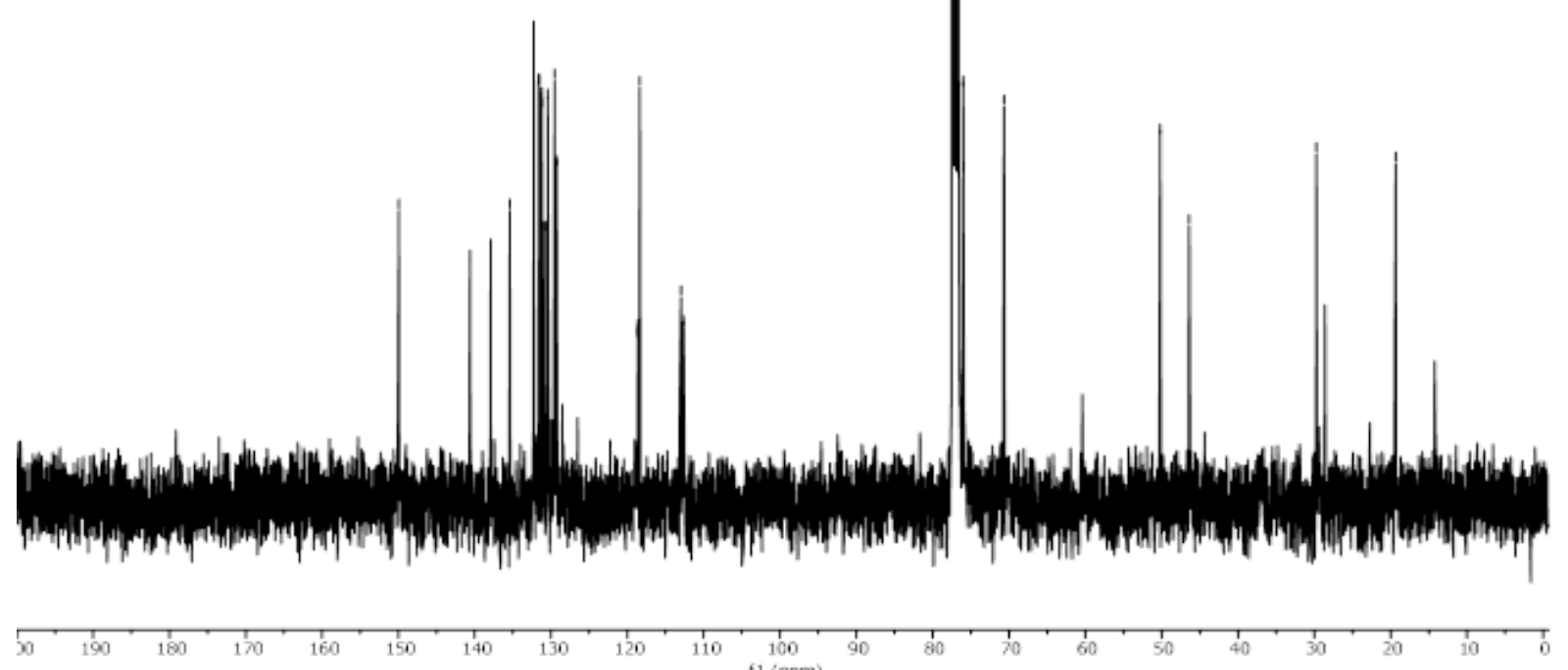
2u, ${ }^{1} \mathbf{H}$ NMR $\left(400 \mathrm{MHz}, \mathrm{CDCl}_{3}\right)$

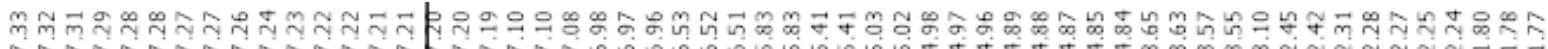

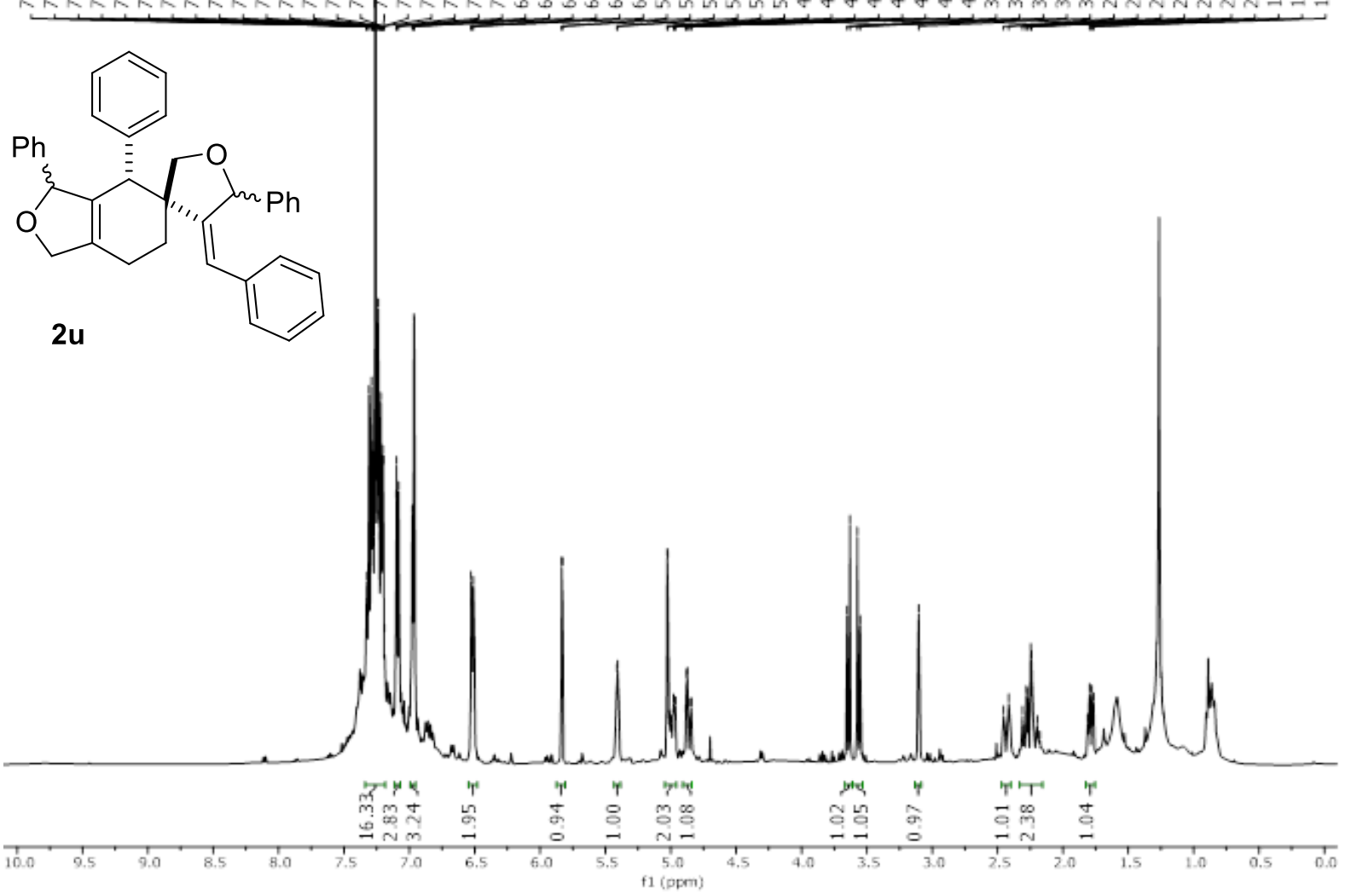

$\mathbf{2 u},{ }^{13} \mathbf{C}\{\mathbf{H}\} \mathbf{N M R}\left(101 \mathrm{MHz}, \mathrm{CDCl}_{3}\right)$

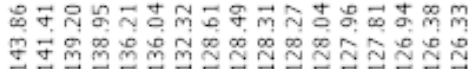

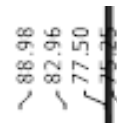

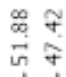

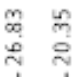

$7=7=7.7$
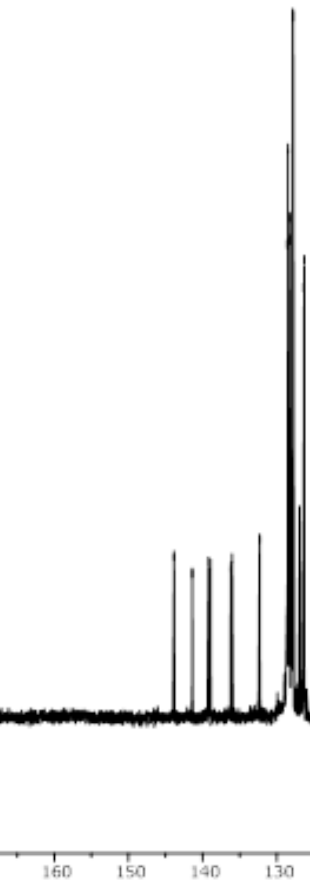

110 100 
2u', ${ }^{1} \mathbf{H}$ NMR $\left(400 \mathrm{MHz}, \mathrm{CDCl}_{3}\right)$

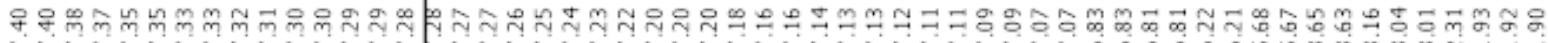

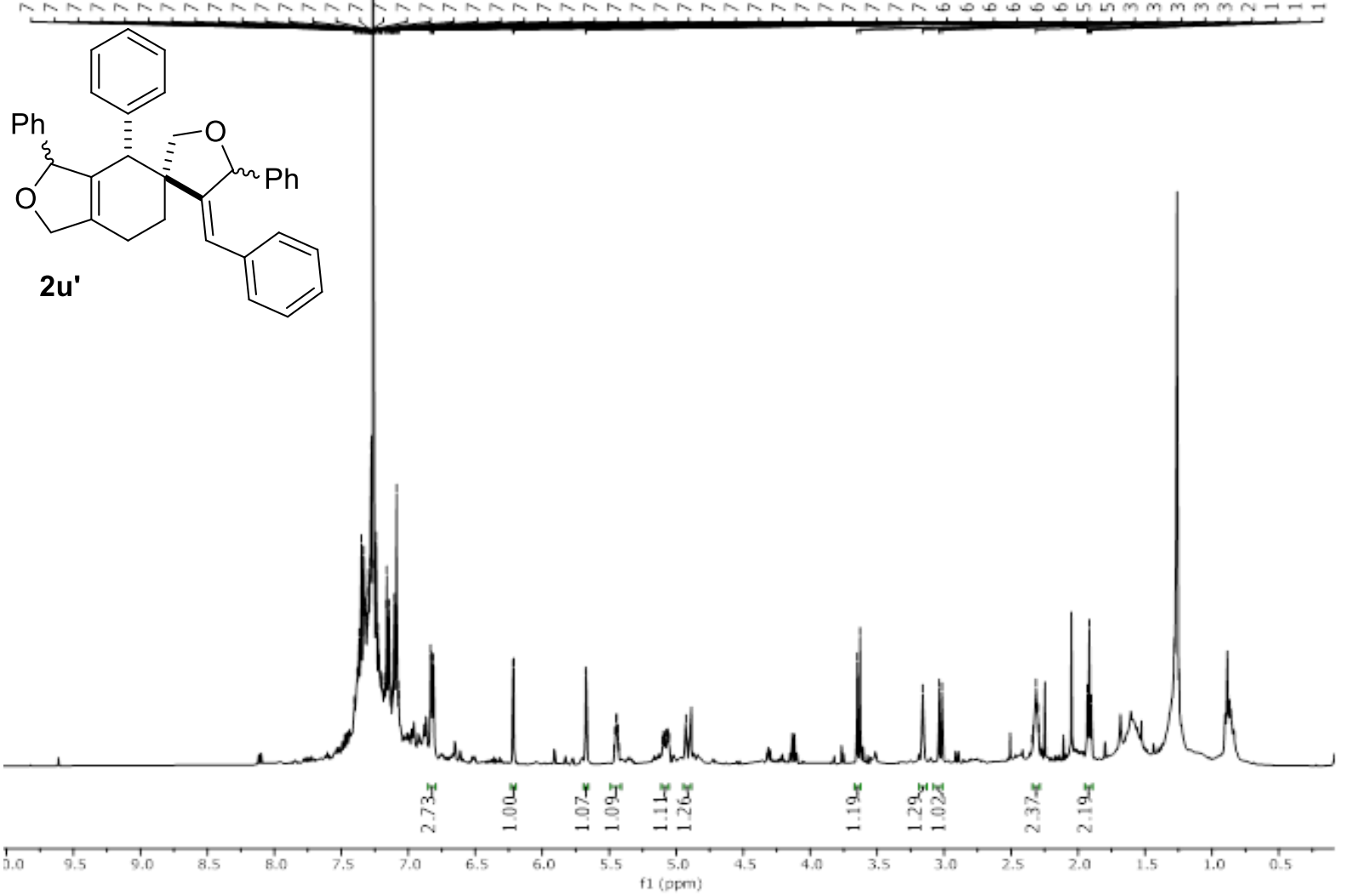

$\mathbf{2} \mathbf{u}^{\prime},{ }^{13} \mathbf{C}\{\mathbf{H}\} \mathbf{N M R}\left(101 \mathrm{MHz}, \mathrm{CDCl}_{3}\right)$

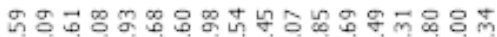

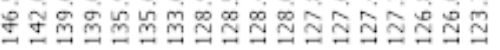

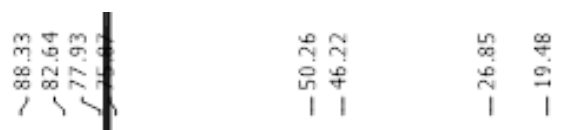
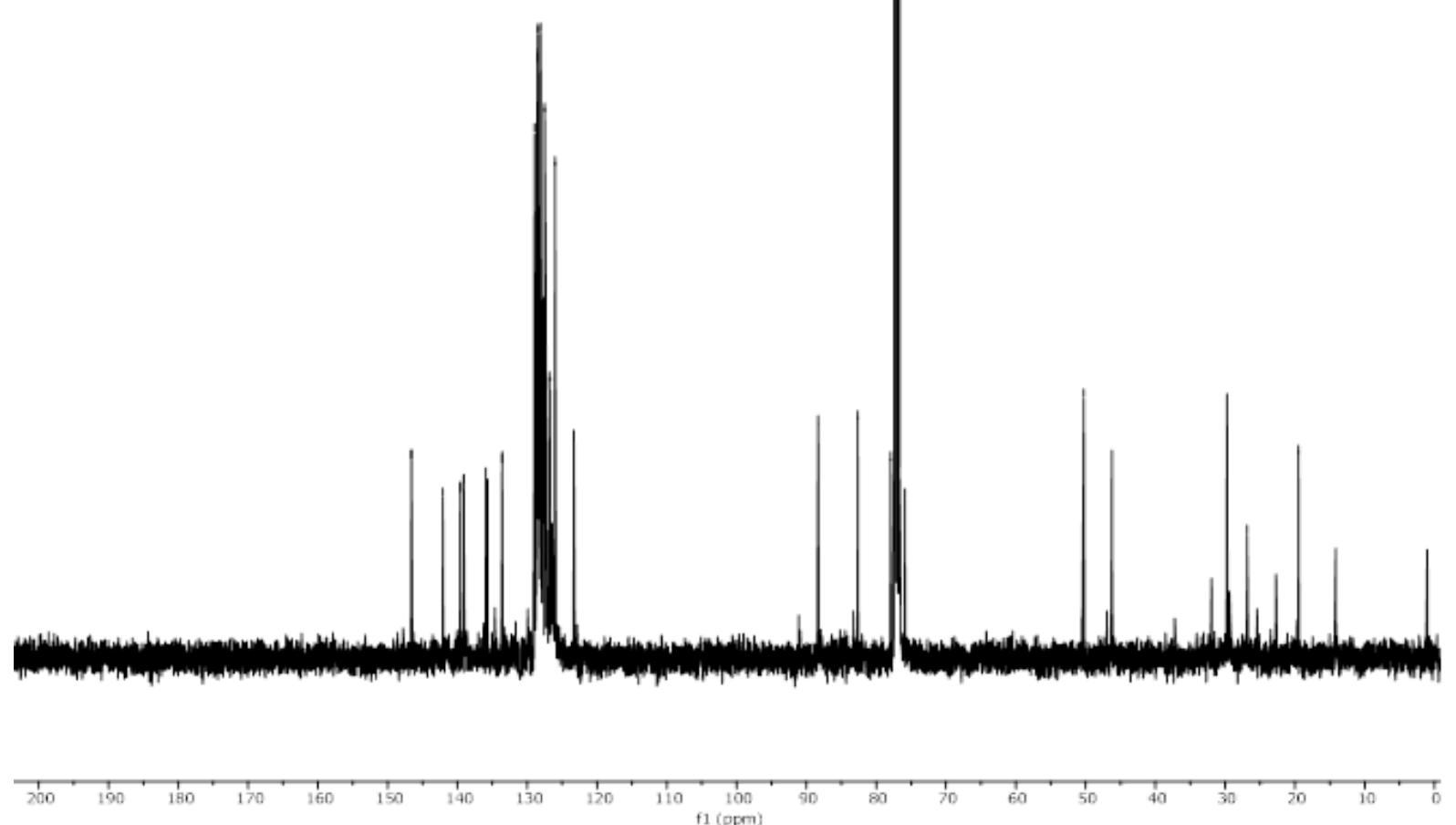
2'v, ${ }^{1} \mathbf{H}$ NMR $\left(400 \mathrm{MHz}, \mathrm{CDCl}_{3}\right)$

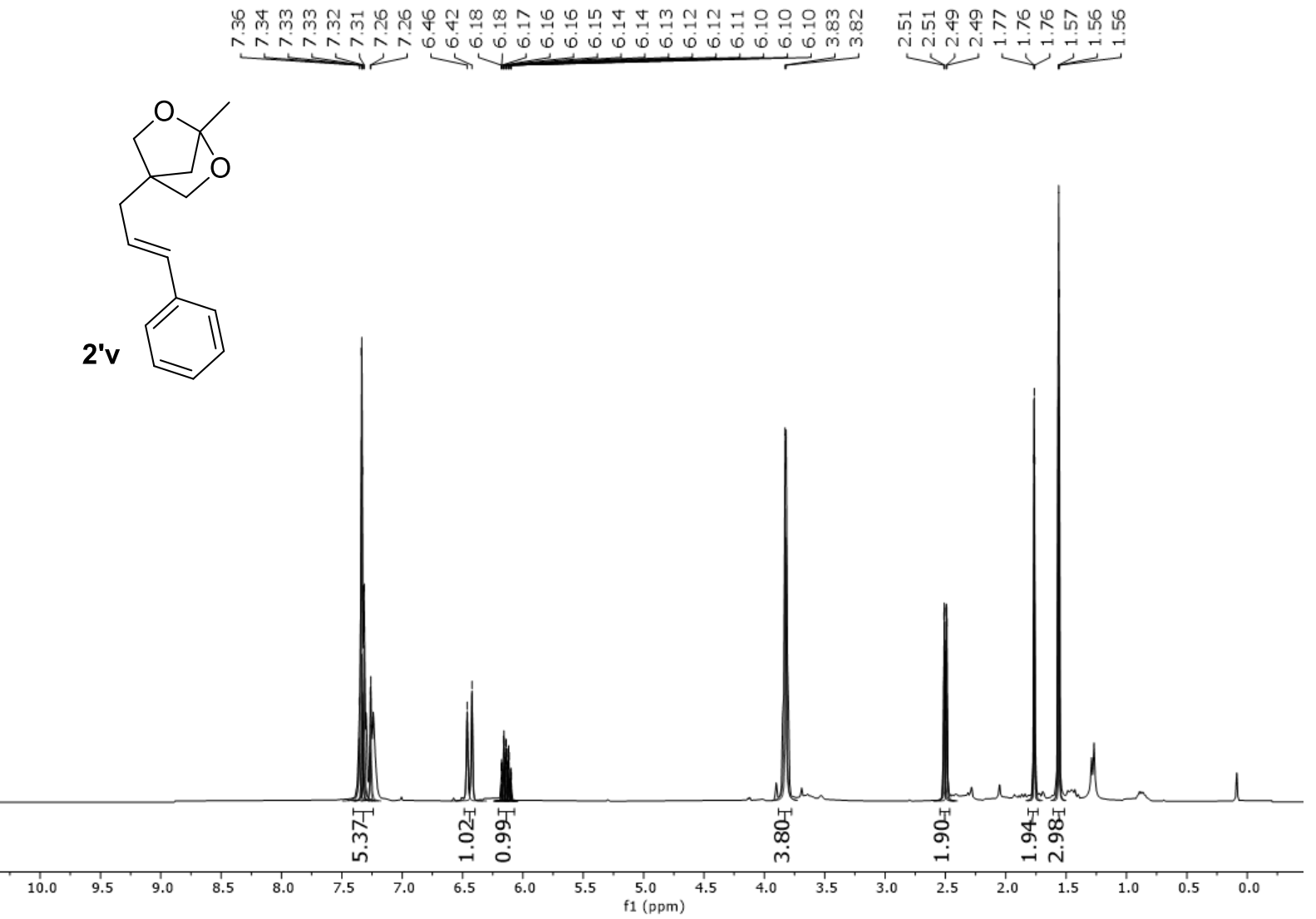


4a, ${ }^{1} \mathbf{H}$ NMR $\left(400 \mathrm{MHz}, \mathrm{CD}_{2} \mathrm{Cl}_{2}\right)$

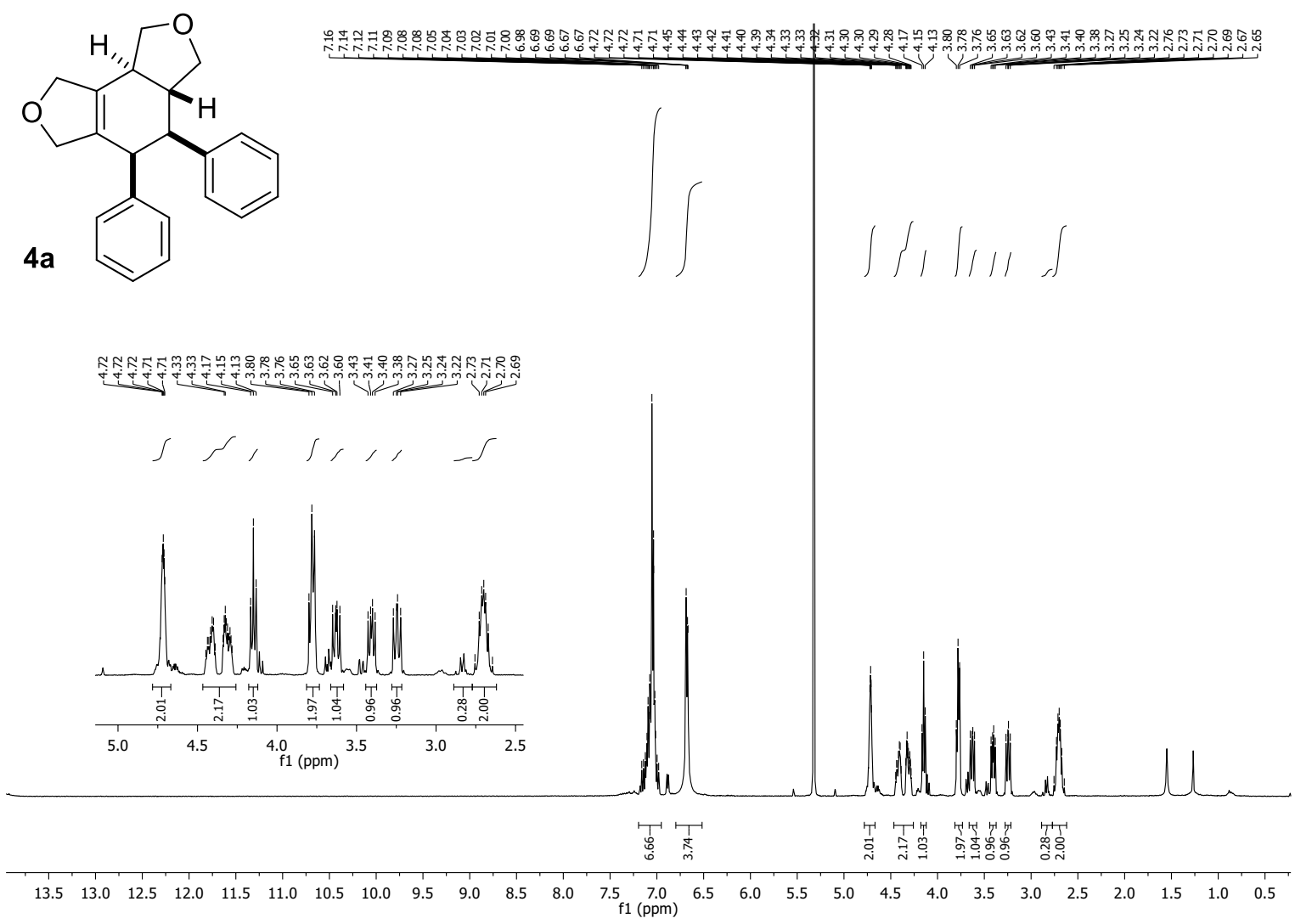

4a, ${ }^{13} \mathbf{C}\{\mathbf{H}\}$ NMR $\left(101 \mathrm{MHz}, \mathrm{CD}_{2} \mathrm{Cl}_{2}\right)$

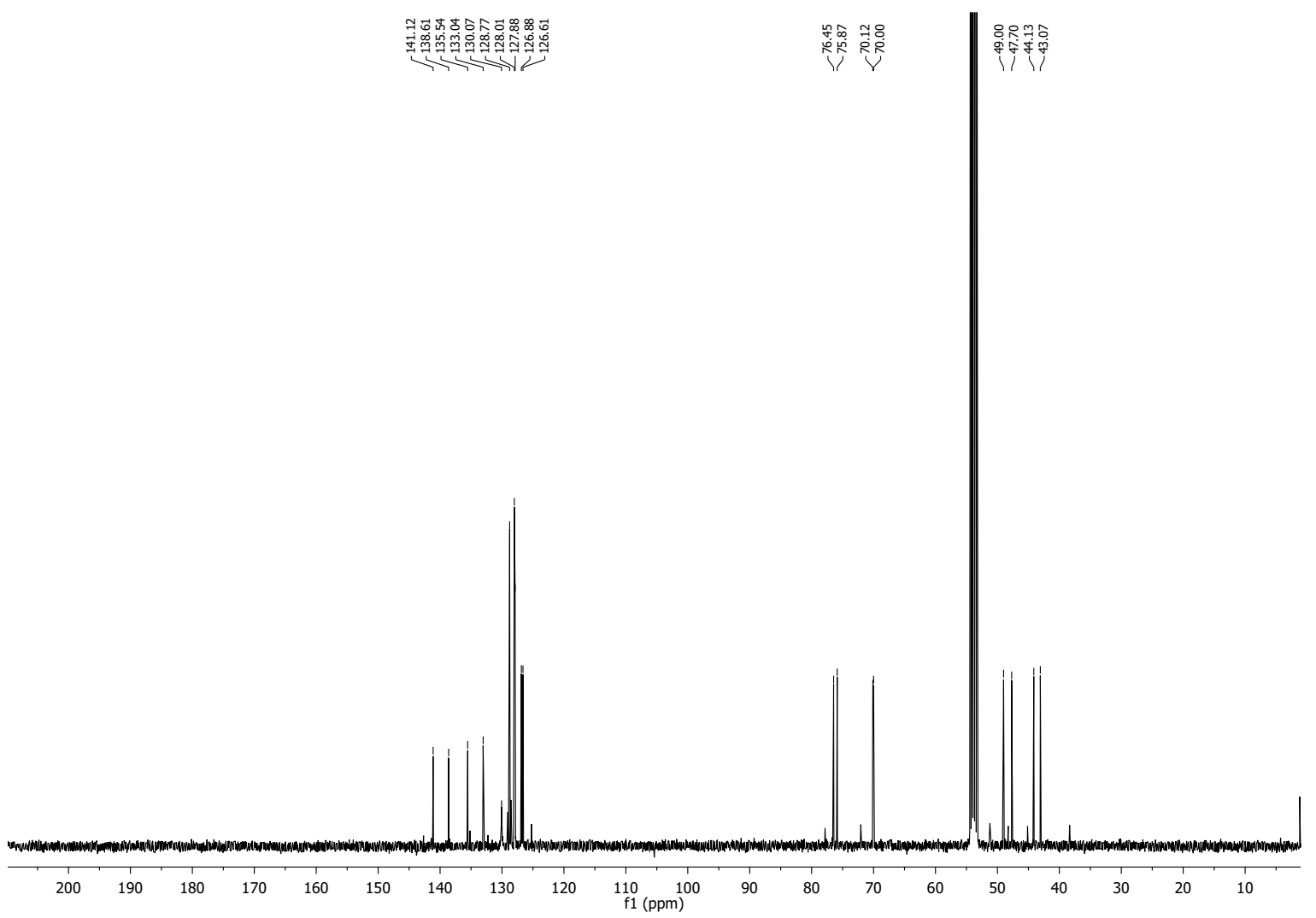


4b, ${ }^{1} \mathbf{H}$ NMR $\left(400 \mathrm{MHz}, \mathrm{CDCl}_{3}\right)$

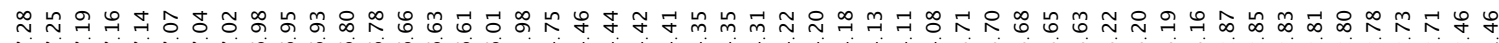

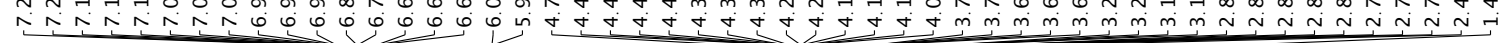<smiles>Cc1ccccc1[C@@H]1[C@@H]2COC[C@H]2C2=C(COC2)[C@H]1c1ccccc1C</smiles>

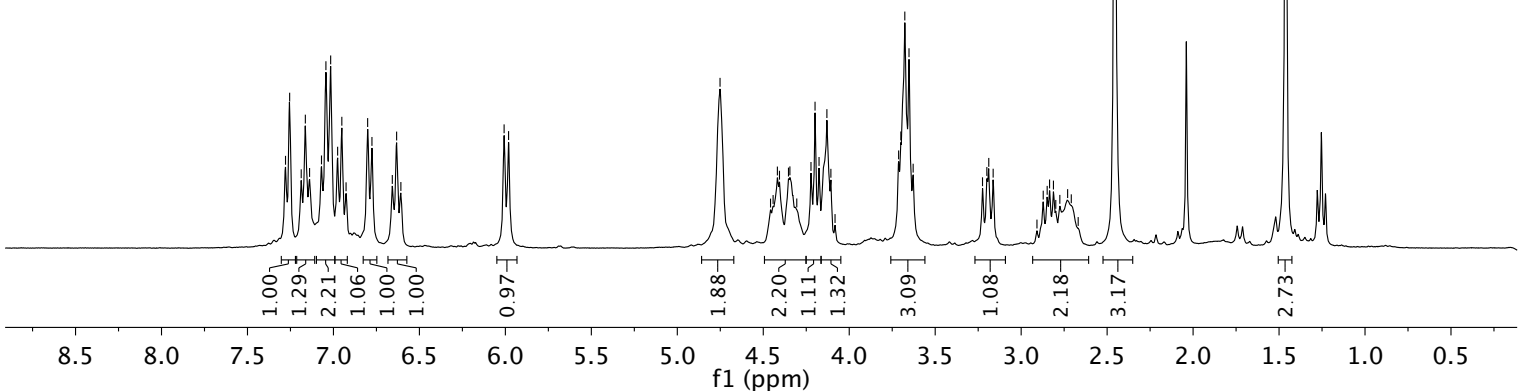

4b, ${ }^{13} \mathbf{C}\{\mathbf{H}\}$ NMR $\left(101 \mathrm{MHz}, \mathrm{CDCl}_{3}\right)$

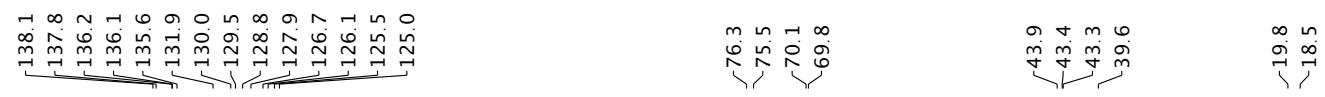

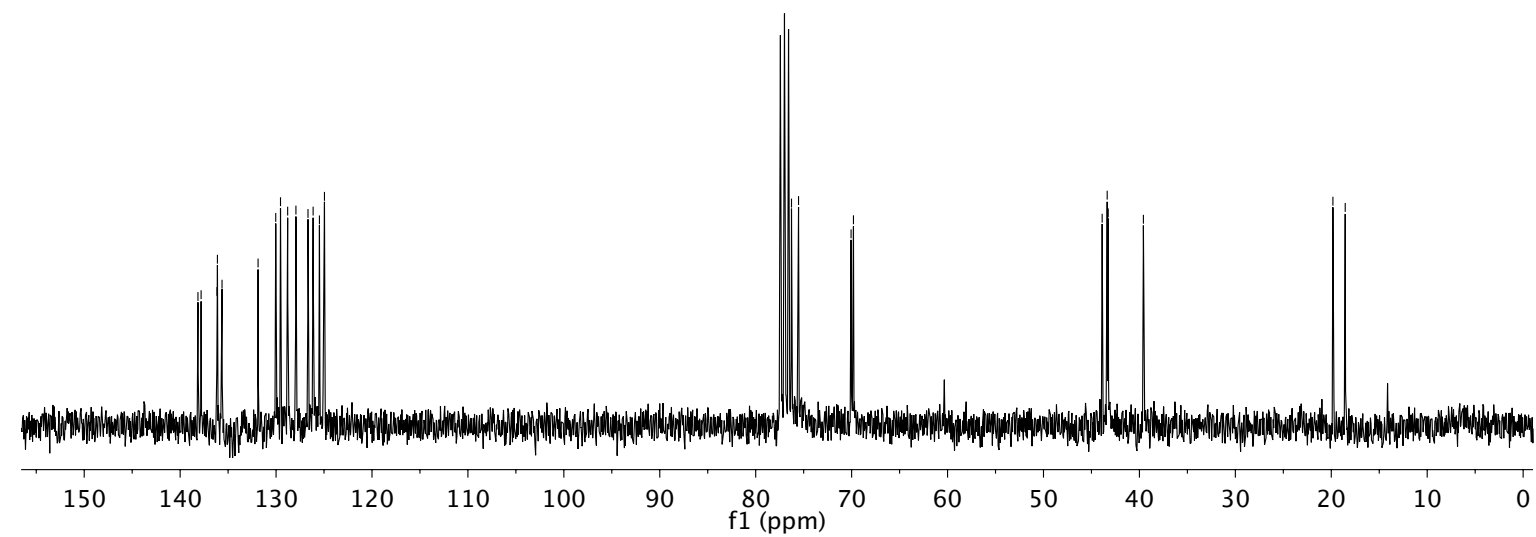


4c, ${ }^{1} \mathbf{H}$ NMR $\left(400 \mathrm{MHz}, \mathrm{CDCl}_{3}\right)$

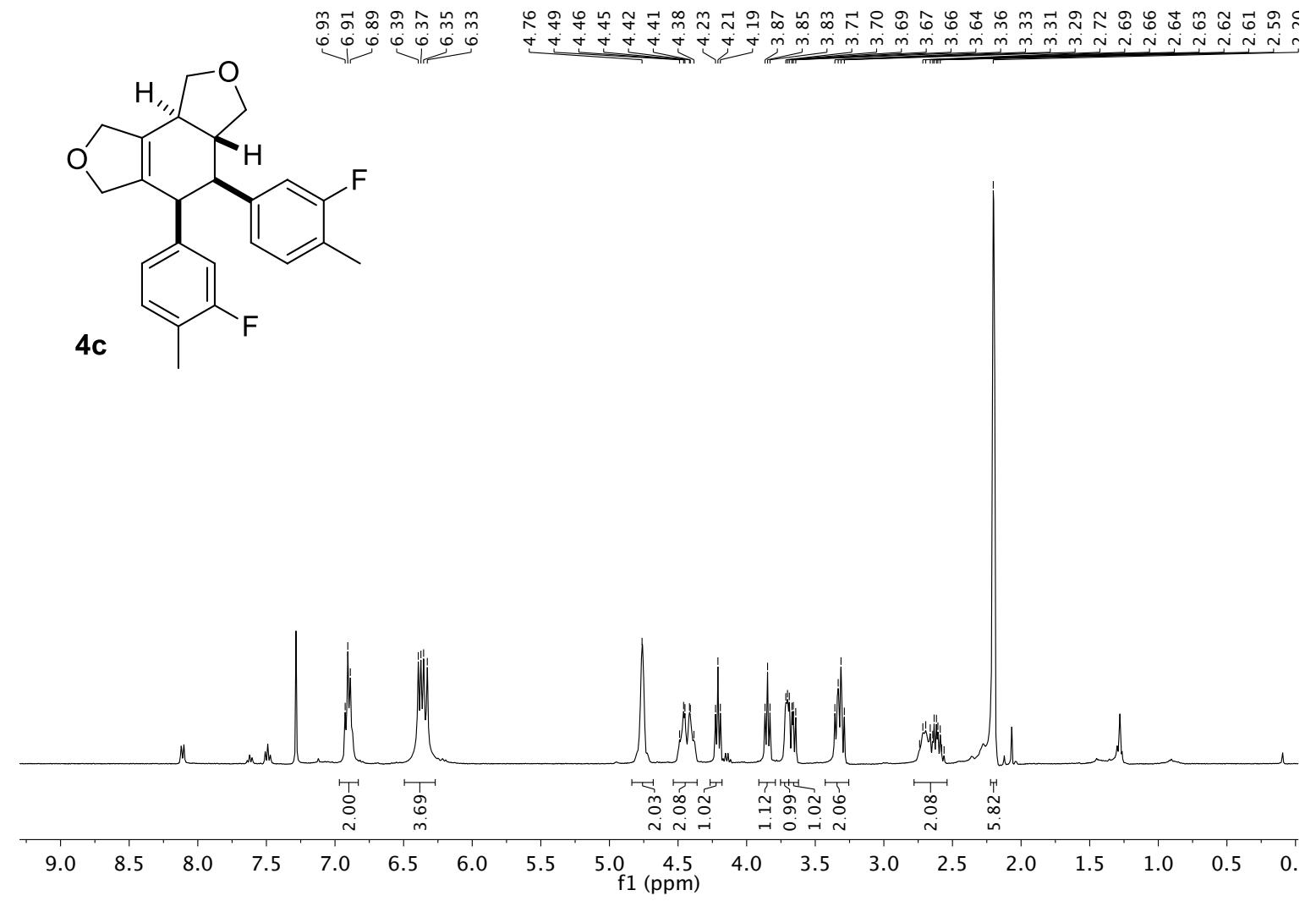

4c, ${ }^{13} \mathbf{C}\{\mathbf{H}\}$ NMR $\left(101 \mathrm{MHz}, \mathrm{CDCl}_{3}\right)$

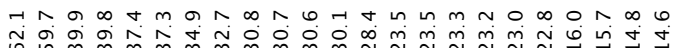

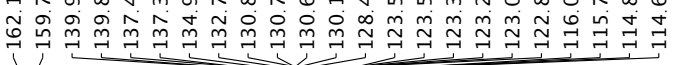

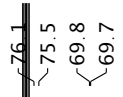

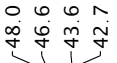

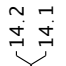

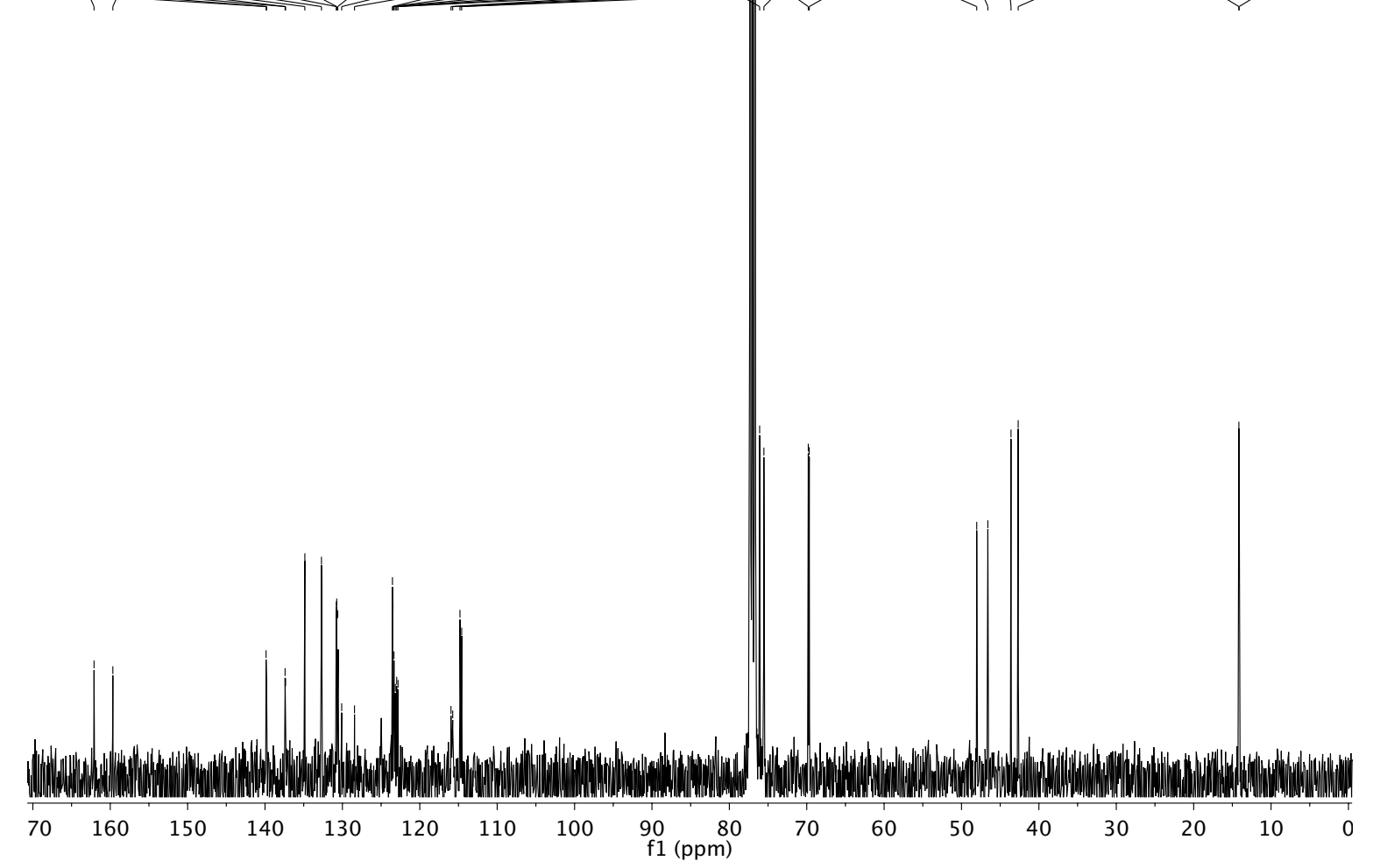


4c, ${ }^{19} \mathbf{F}\{\mathbf{H}\}$ NMR $\left(376 \mathrm{MHz}, \mathrm{CDCl}_{3}\right)$

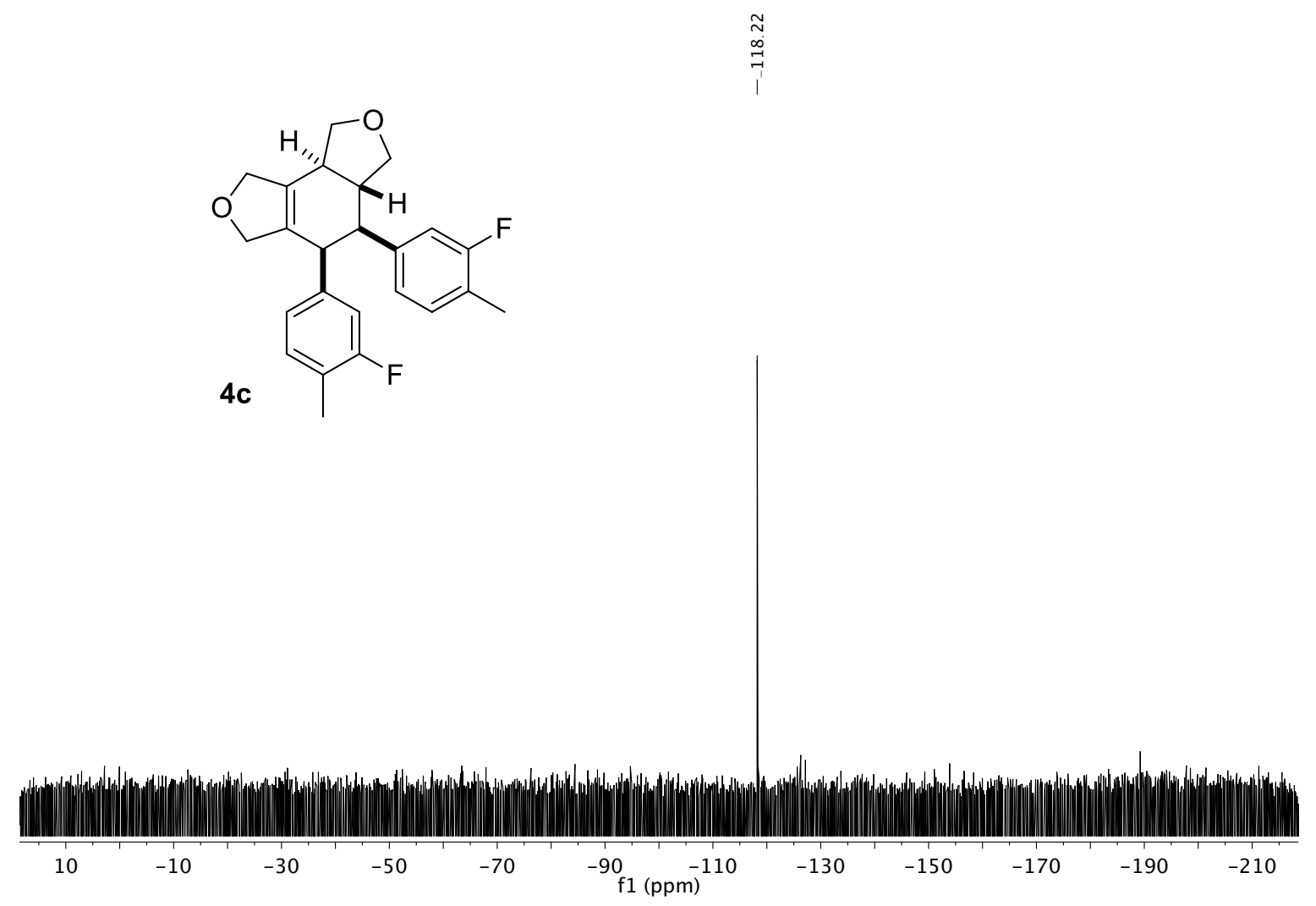


4d, ${ }^{1}$ H NMR $\left(400 \mathrm{MHz}\right.$, Acetone- $\left.d_{6}\right)$

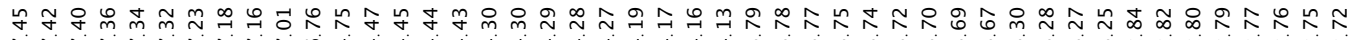

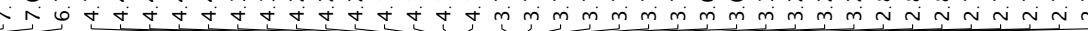

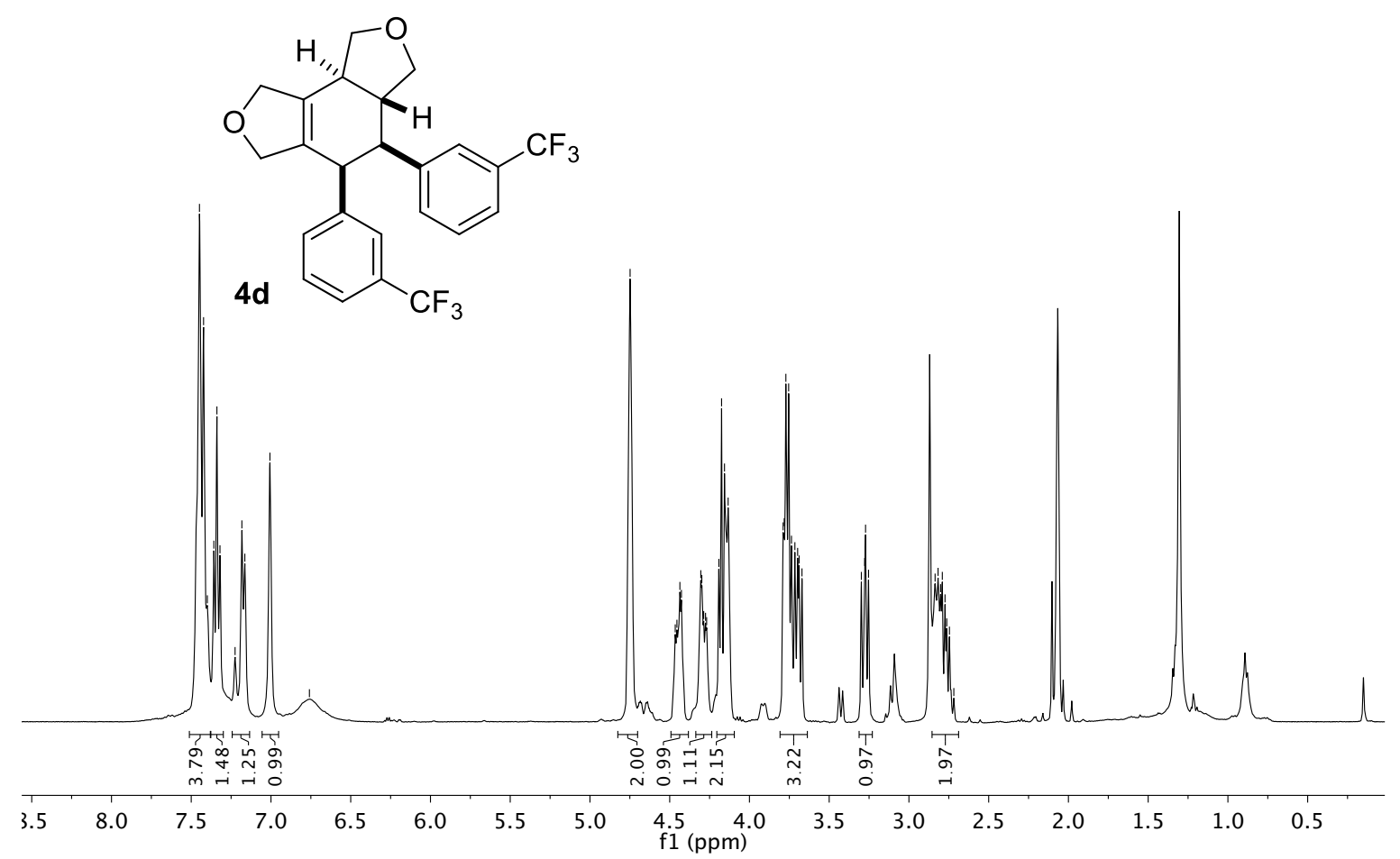

4d, ${ }^{13} \mathbf{C}\{\mathbf{H}\}$ NMR $\left(101 \mathrm{MHz}\right.$, Acetone- $\left.d_{6}\right)$

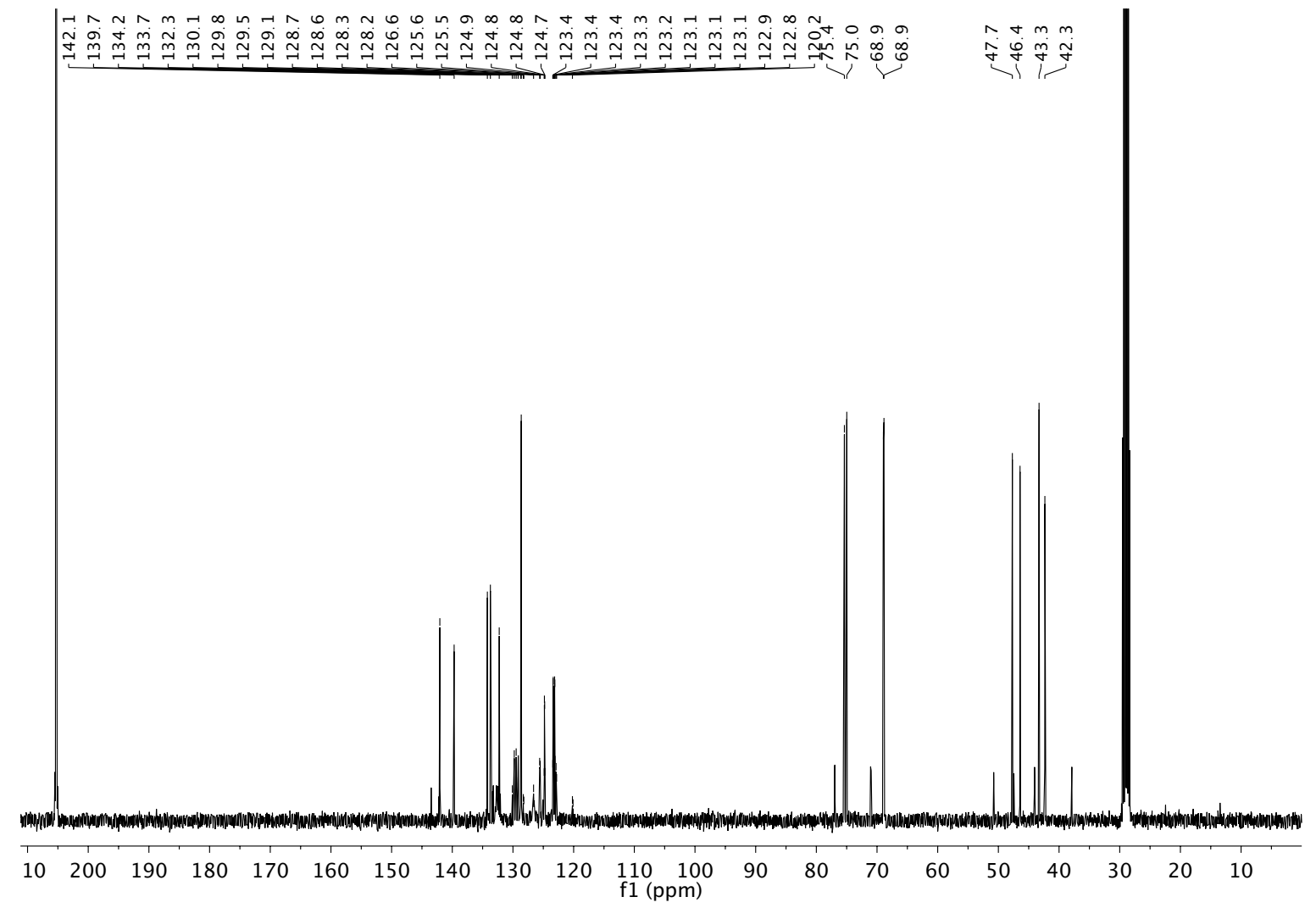


4d, ${ }^{19} \mathbf{F}\{\mathbf{H}\}$ NMR (565 MHz, Acetone-d 6 )

$\overrightarrow{0}$
0
1<smiles>FC(F)(F)c1cccc([C@H]2[C@H](c3cccc(C(F)(F)F)c3)C3=C(COC3)[C@H]3COC[C@@H]32)c1</smiles>

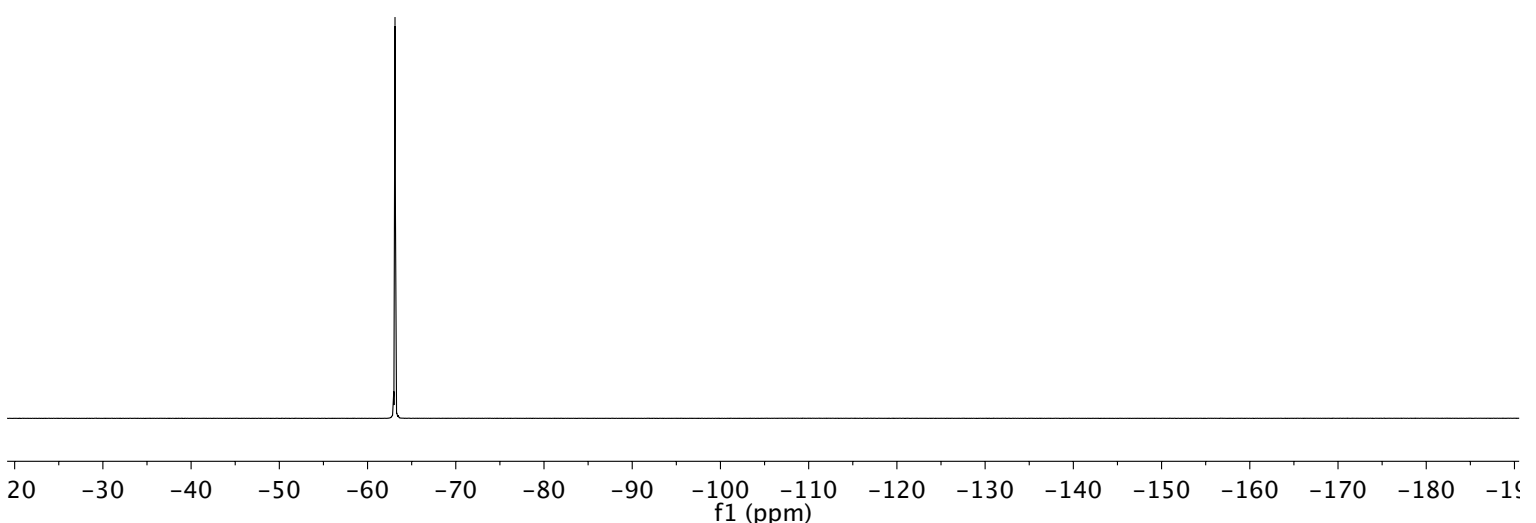


4e, ${ }^{1} \mathbf{H}$ NMR $\left(400 \mathrm{MHz}, \mathrm{CDCl}_{3}\right)$

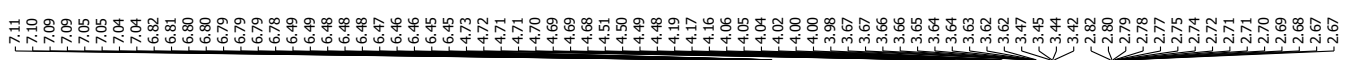

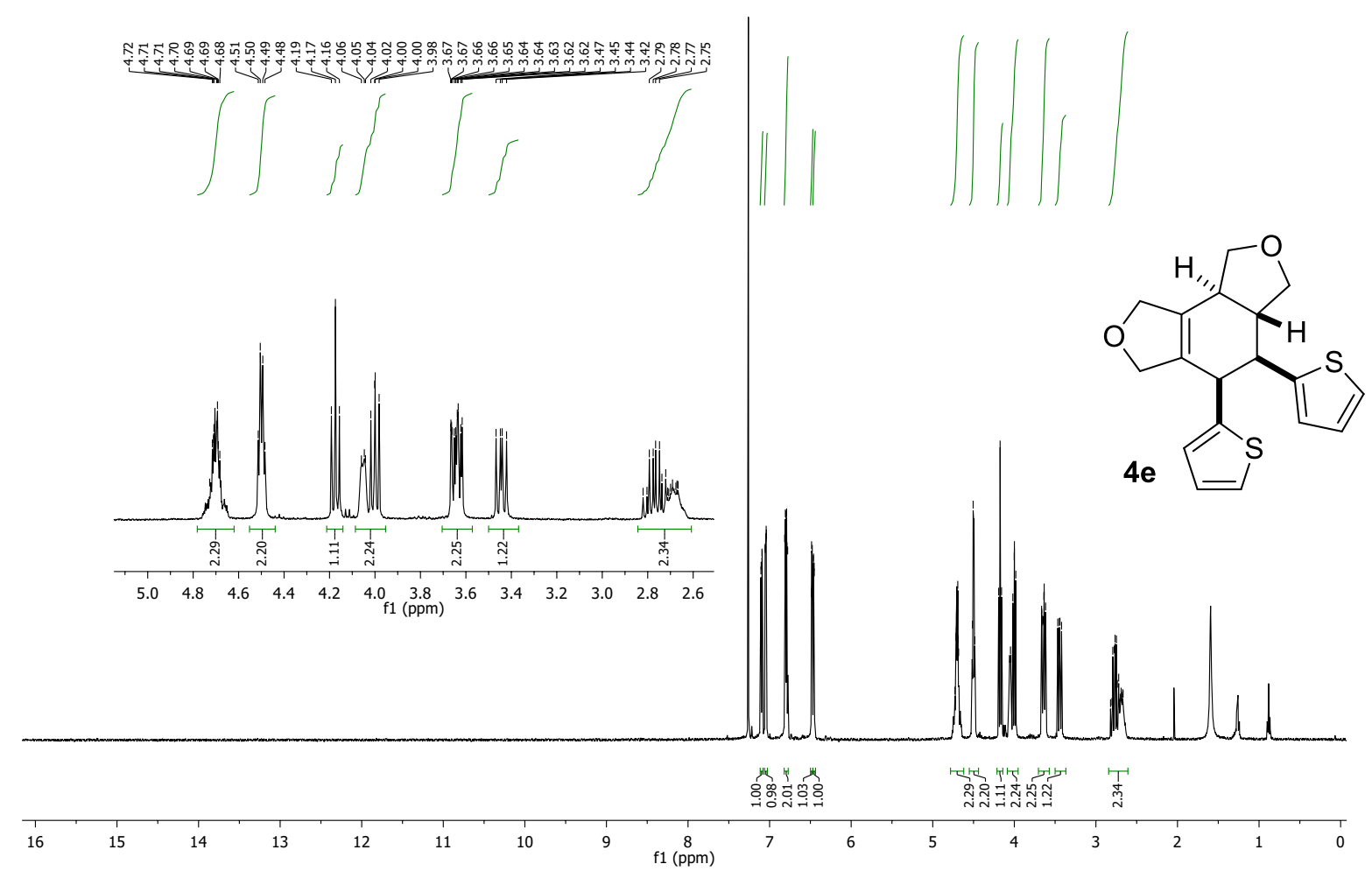

4e, ${ }^{13} \mathbf{C}\{\mathbf{H}\}$ NMR $\left(101 \mathrm{MHz}, \mathrm{CDCl}_{3}\right)$

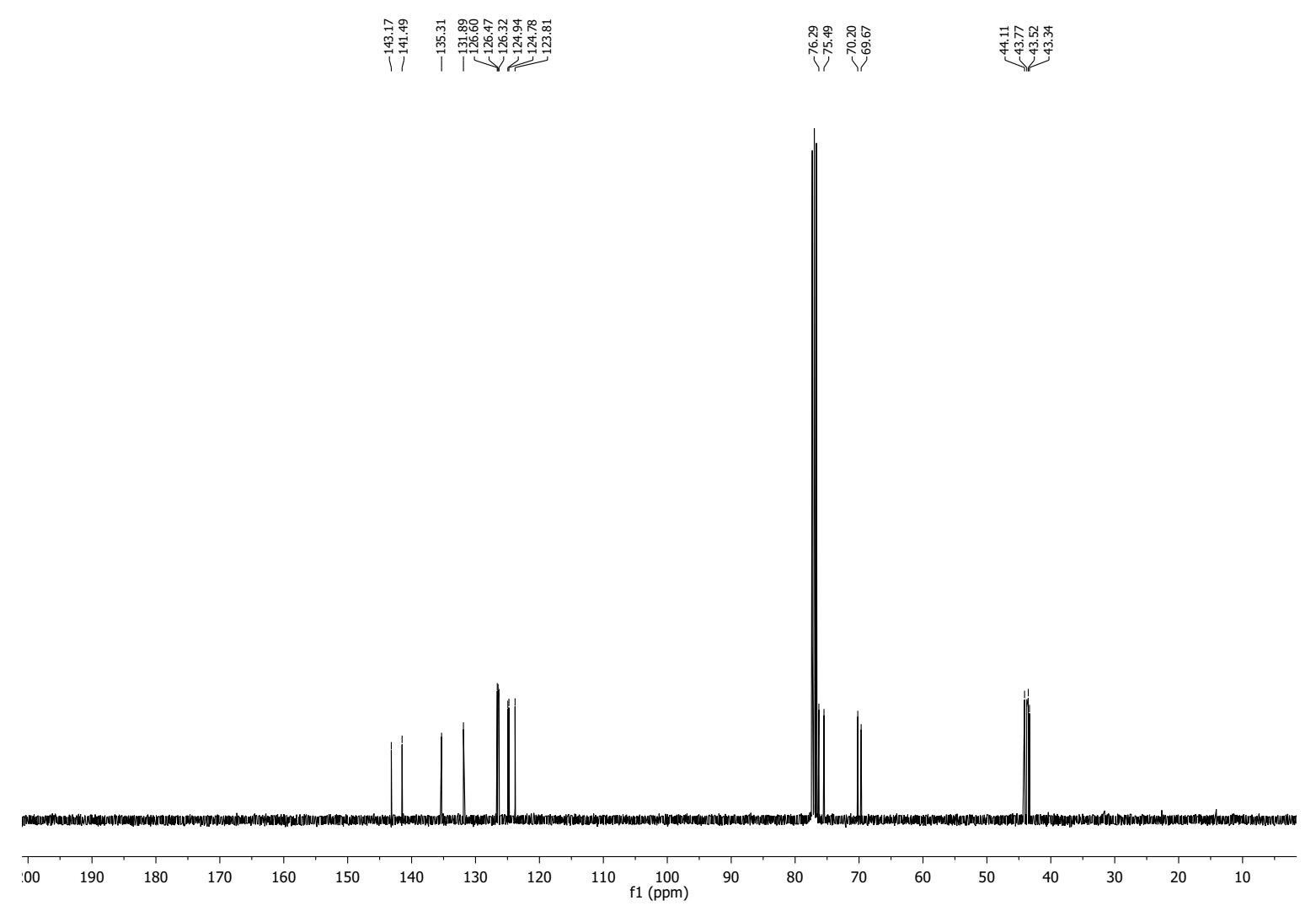




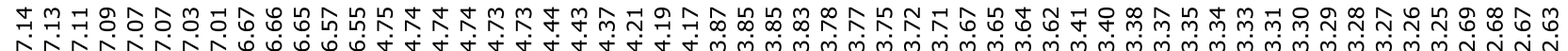

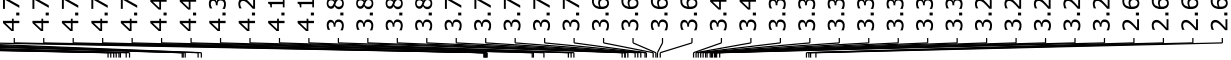

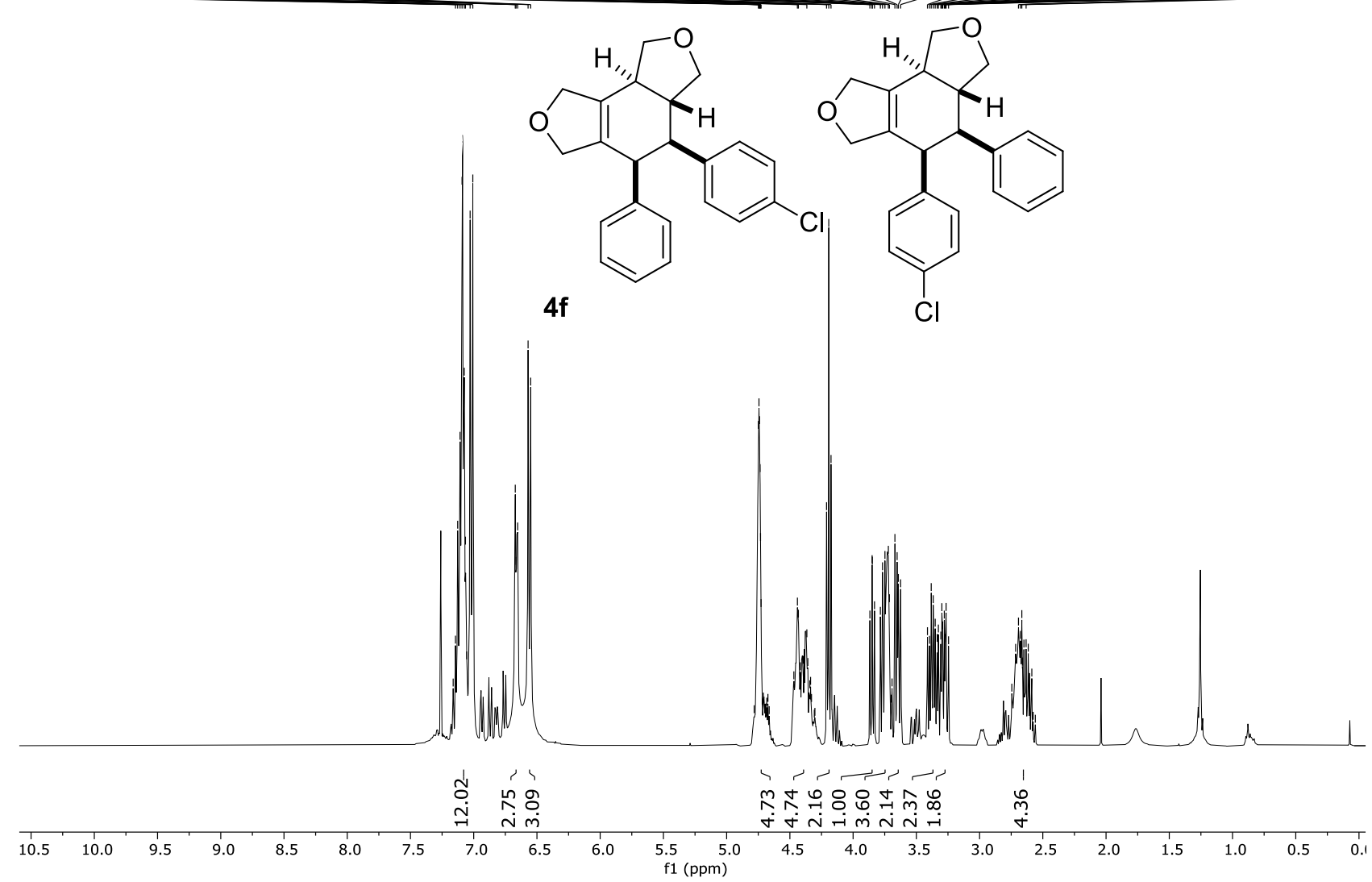

4f/24, ${ }^{13} \mathbf{C}\{\mathbf{H}\}$ NMR $\left(101 \mathrm{MHz}, \mathrm{CDCl}_{3}\right)$

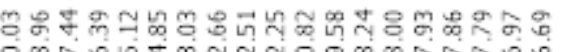

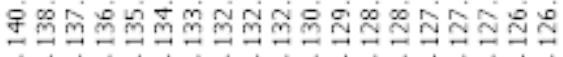

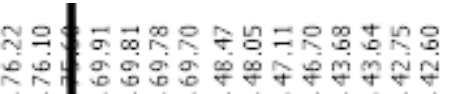

(1)

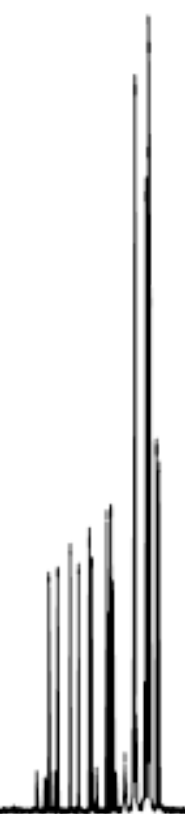

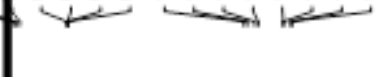

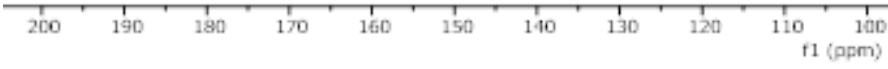


4g", ${ }^{1} \mathbf{H}$ NMR $\left(400 \mathrm{MHz}, \mathrm{CDCl}_{3}\right)$

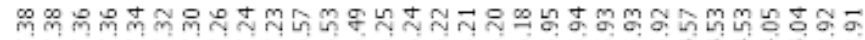

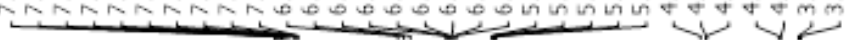

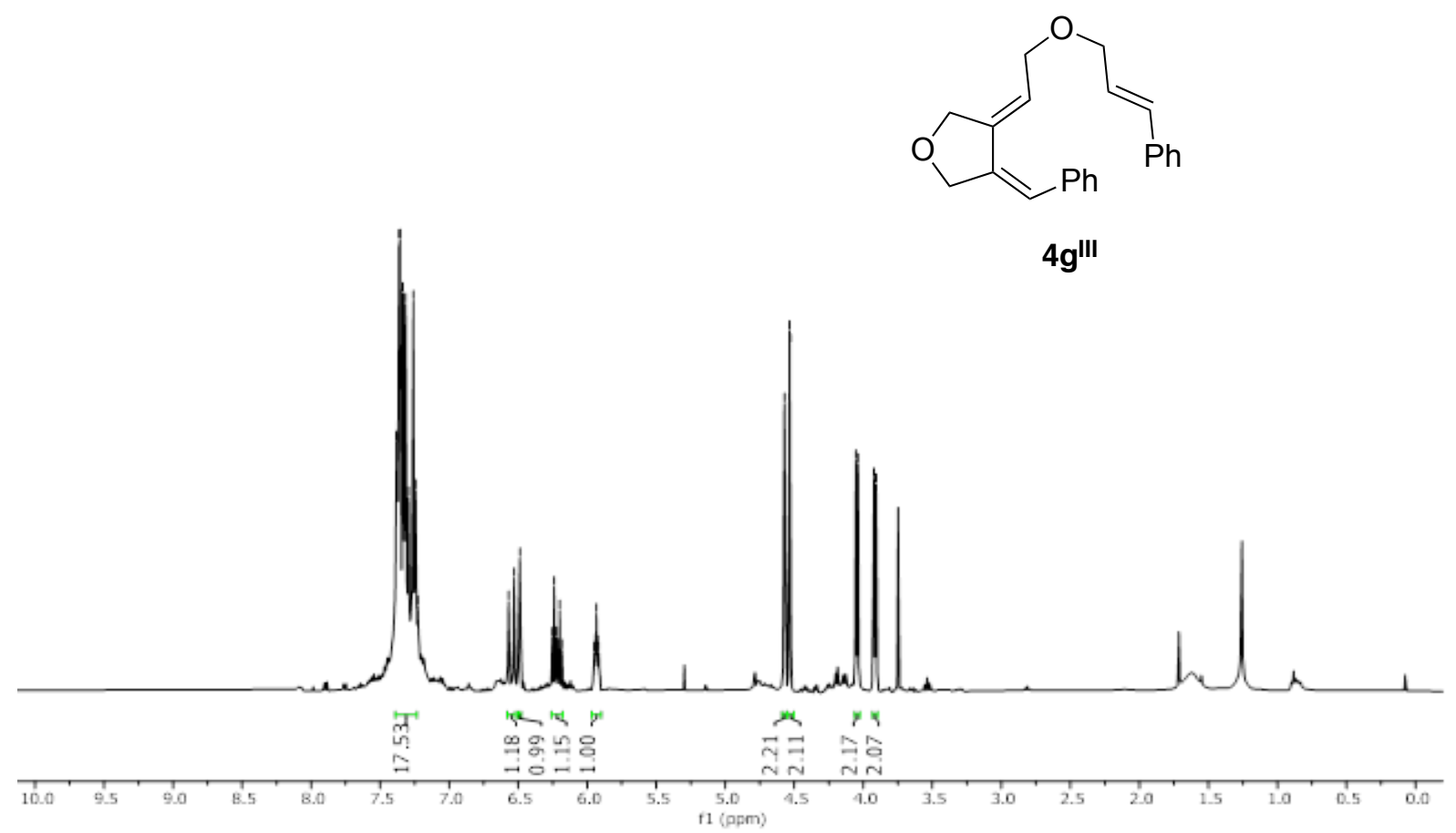

4g,", ${ }^{13} \mathbf{C}\{\mathbf{H}\}$ NMR $\left(101 \mathrm{MHz}, \mathrm{CDCl}_{3}\right)$

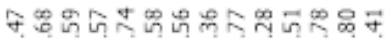

舟品品品
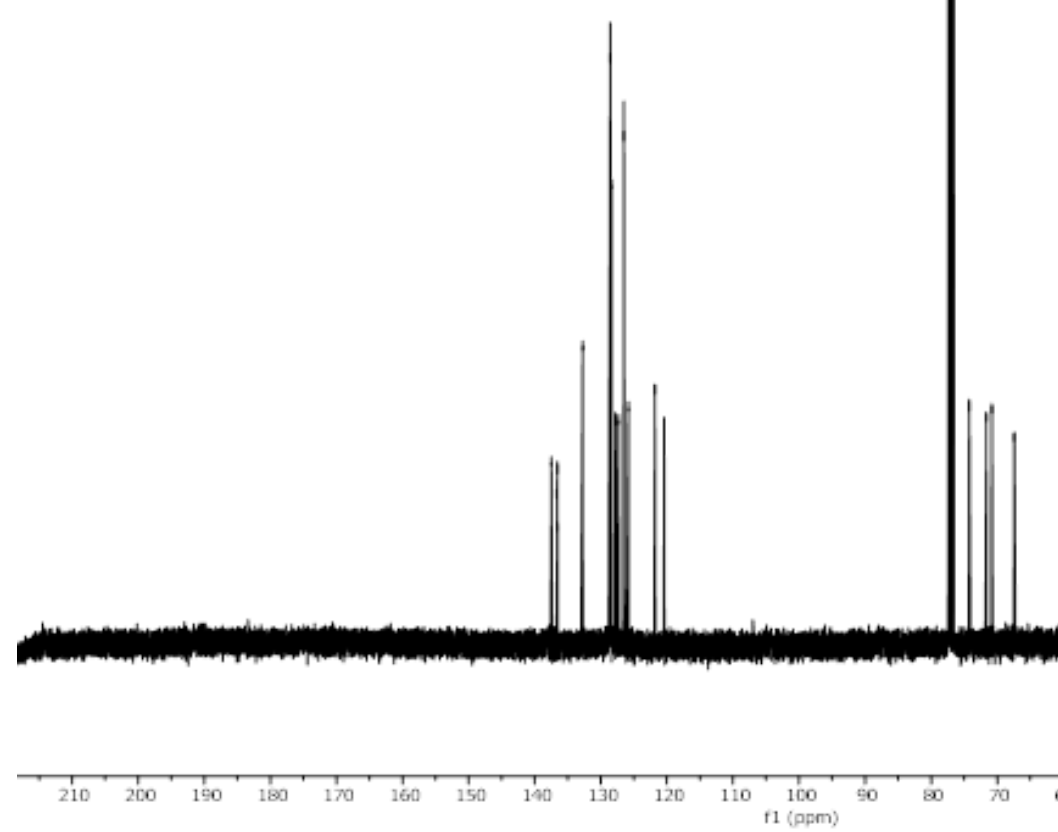
4g"', NOESY NMR $\left(400 \mathrm{MHz}, \mathrm{CDCl}_{3}\right)$
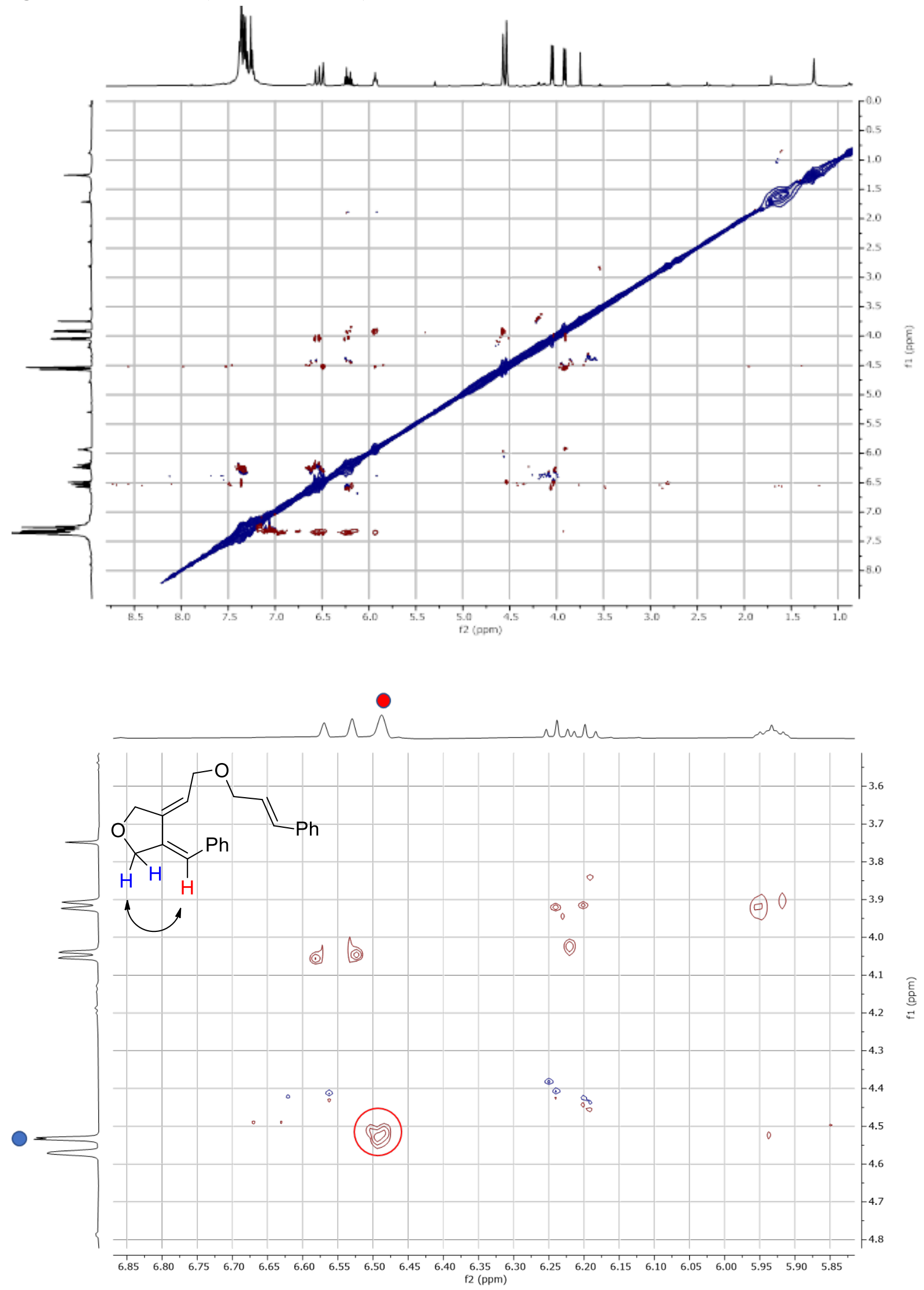
4g, ${ }^{1} \mathbf{H}$ NMR (400 MHz, $\left.\mathrm{CDCl}_{3}\right)$

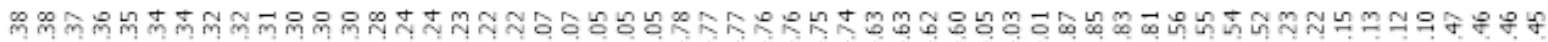

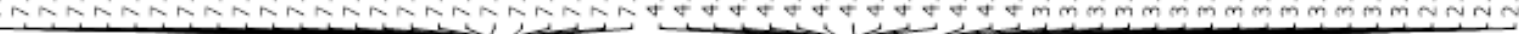

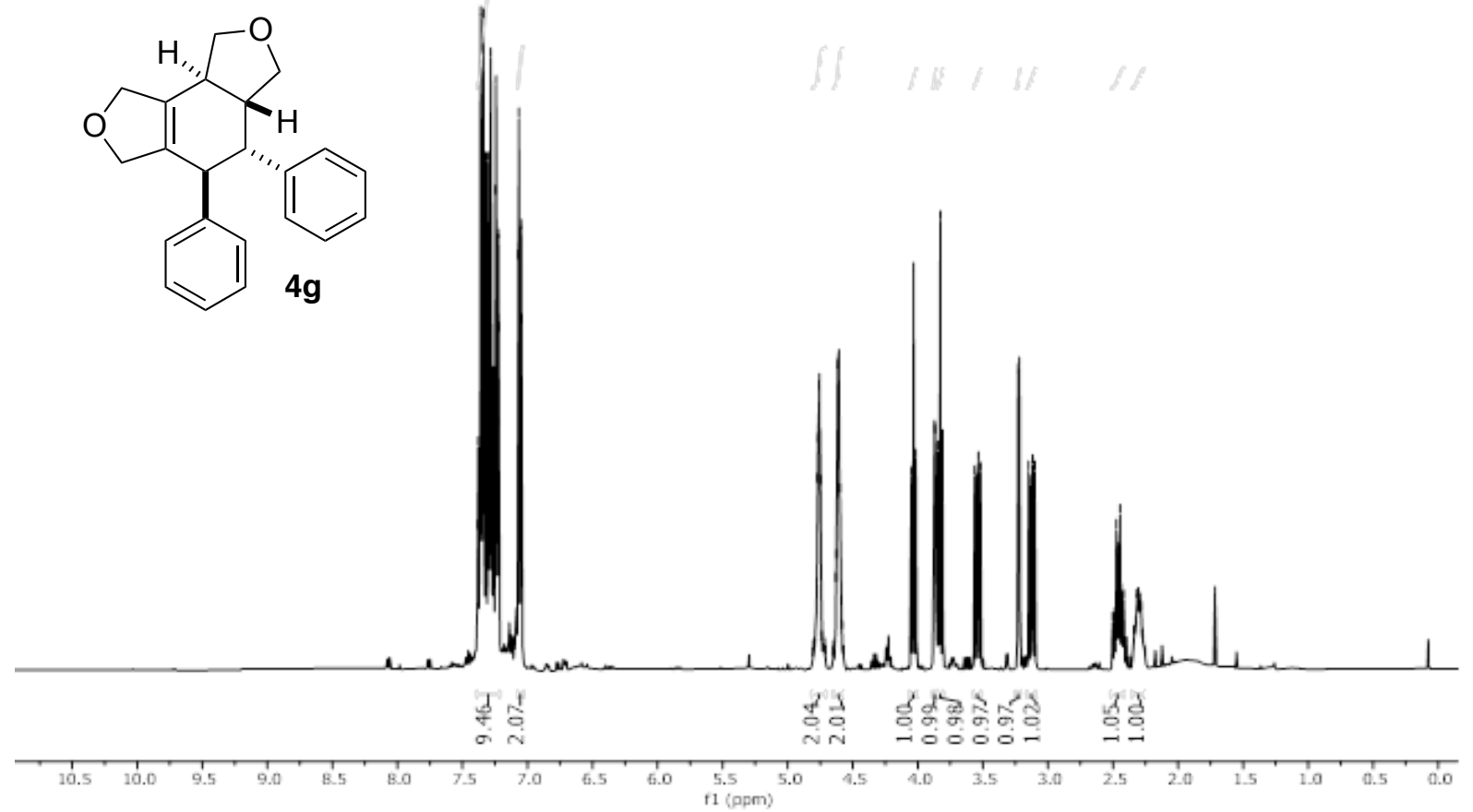

4g, ${ }^{13} \mathbf{C}\{\mathbf{H}\}$ NMR $\left(101 \mathrm{MHz}, \mathrm{CDCl}_{3}\right)$

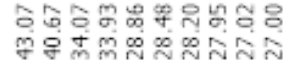

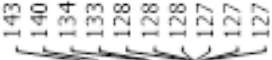

요 $\infty m$

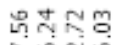

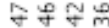

it?

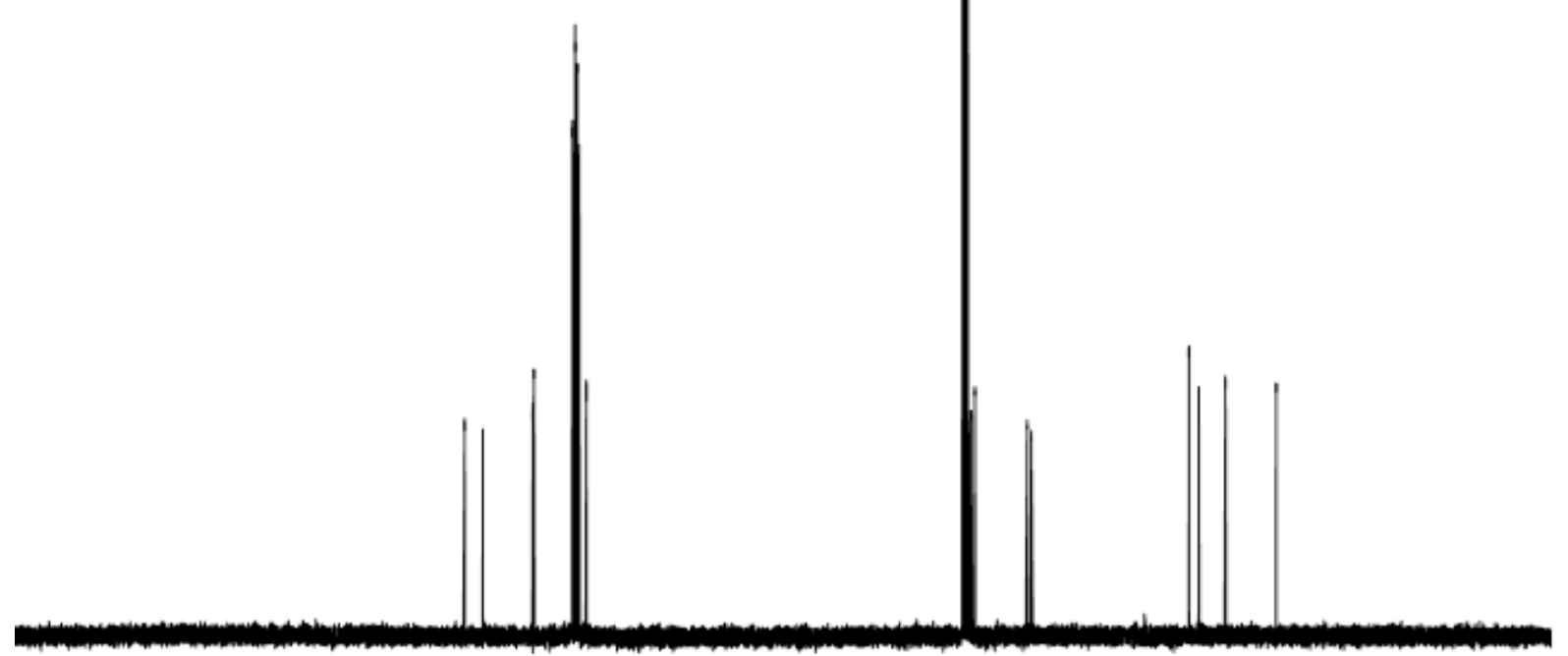

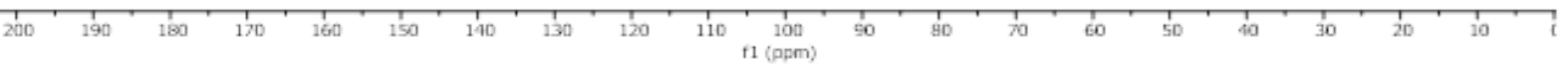


4g, NOESY NMR $\left(400 \mathrm{MHz}, \mathrm{CDCl}_{3}\right)$
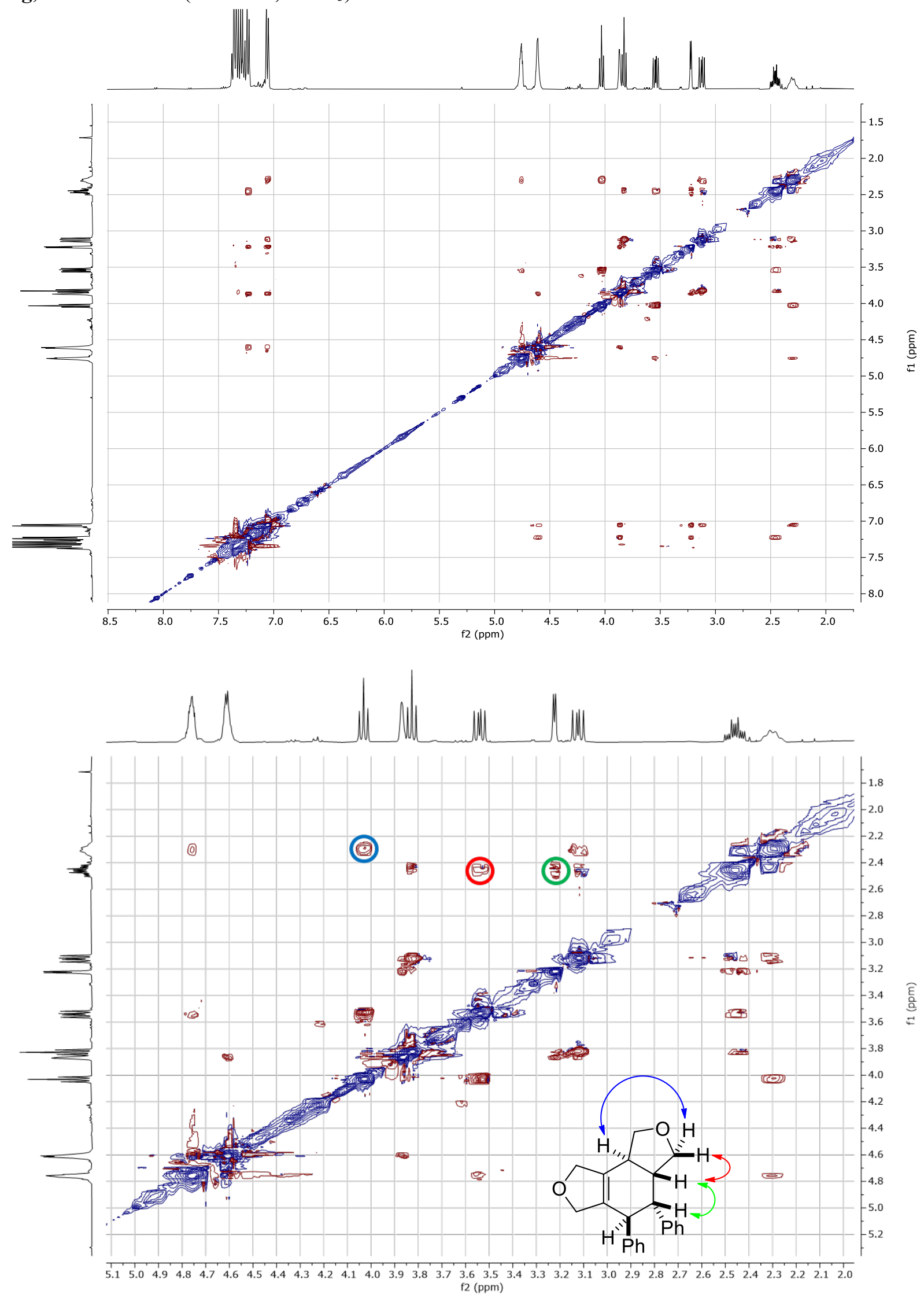